\title{
ASSOCIATIONS BETWEEN WATER-QUALITY TRENDS IN NEW JERSEY STREAMS AND DRAINAGE-BASIN CHARACTERISTICS, 1975-86
}

by Keith W. Robinson, Timothy R. Lazaro, and Connie Pak

\section{U.S. GEOLOGICAL SURVEY}

Water-Resources Investigations Report 96-4119

West Trenton, New Jersey 


\title{
U.S. DEPARTMENT OF THE INTERIOR
}

Bruce Babbitt, Secretary

\author{
U.S. GEOLOGICAL SURVEY
}

Gordon P. Eaton, Director

For additional information write to:

District Chief

U.S. Geological Survey

810 Bear Tavern Road, Suite 206

West Trenton, NJ 08628
Copies of this report can be obtained from:

U.S. Geological Survey Branch of Information Services Box 25286

Denver, CO 80225-0286 


\section{CONTENTS}

Page

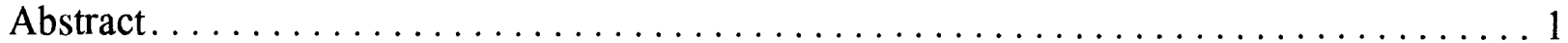

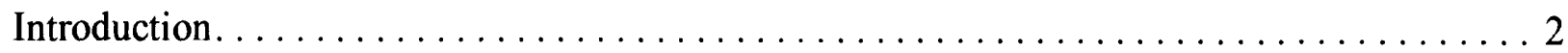

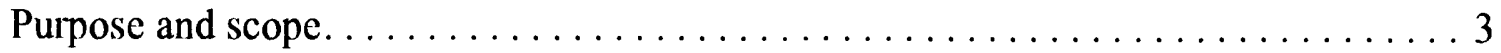

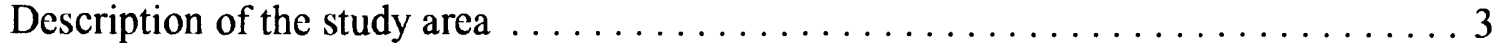

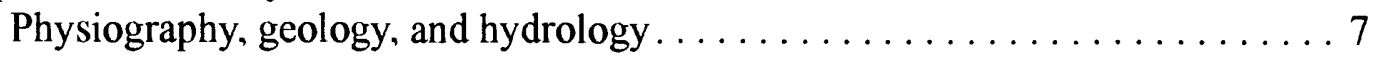

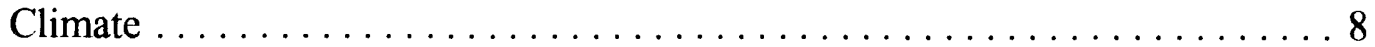

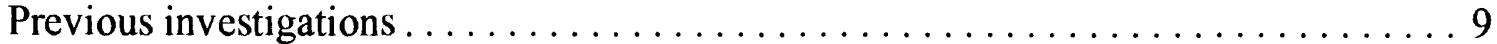

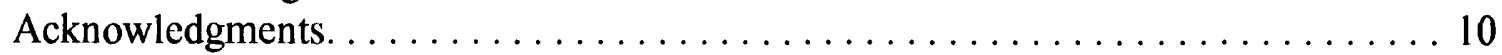

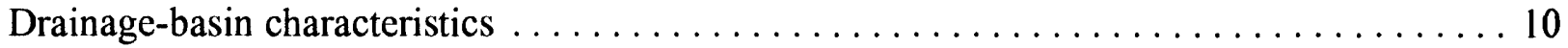

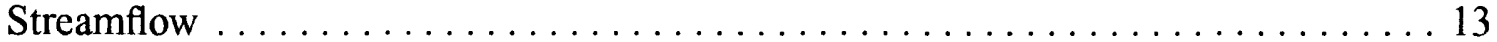

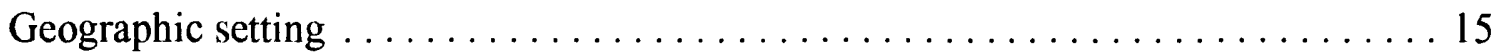

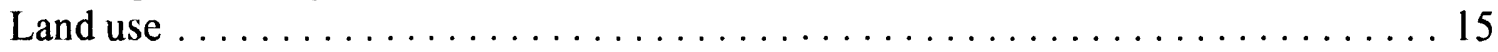

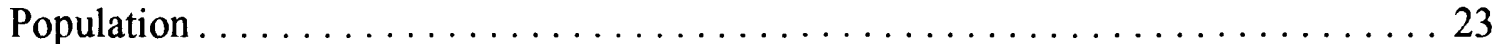

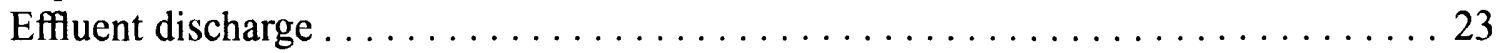

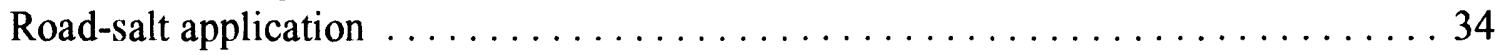

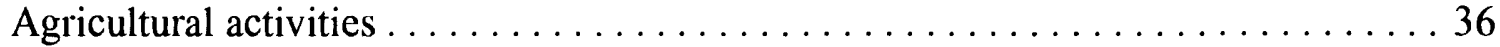

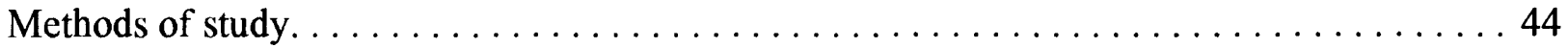

Statistical methods for associating water-quality trends and drainage-basin characteristics . . . . . . . . . . . . . . . . . . . . . . . . . 44

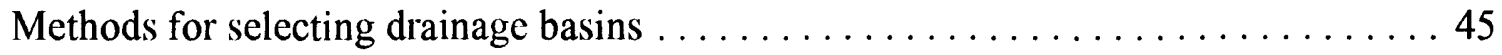

Associations between water-quality trends and drainage-basin characteristics . . . . . . 47

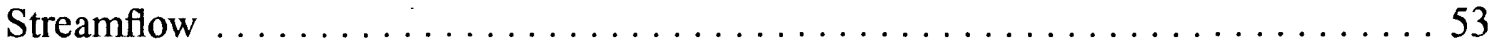

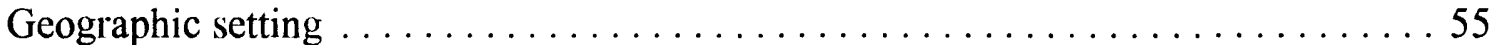

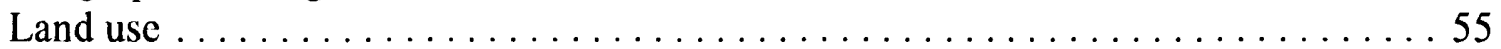

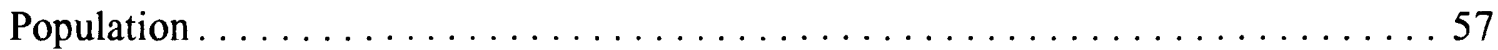

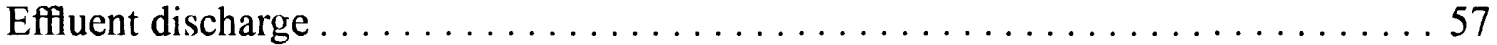

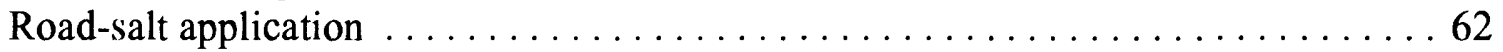

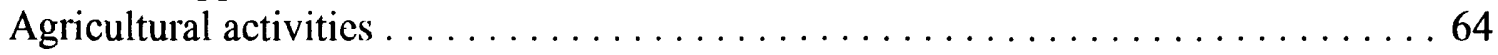

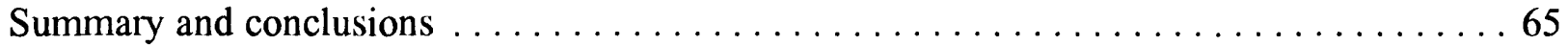

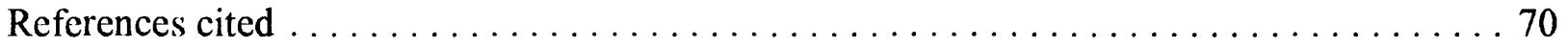

\section{ILLUSTRATIONS}

Figure 1. Map showing location of stream water-quality-monitoring stations in New Jersey and corresponding drainage basins used in the study . . . . . . . 4

2-11. Maps showing:

2. Locations of New Jersey stream-monitoring stations and detected trends in instantancous streamflow, water years 1976-86 . . . . . . . . 17 .

3. Dominant land use in the 60 New Jersey drainage basins studied: (A) entire drainage basin, (B) area 3.1 miles upstream from the monitoring station, and $(\mathrm{C})$ area 0.62 miles upstream from the monitoring station 


\section{ILLUSTRATIONS--Continued}

Page

Figures 2-17. Maps showing--Continued:

4. Estimated 1986 population density in the New Jersey drainage basins studied .................................. 25

5. Yields of (A) discharge, (B) biochemical oxygen demand (5-day), and (C) total suspended solids for effluent released by wastewater-treatment facilities in the New Jersey drainage basins studied . . . . . . . . . 32

6. Percentage of yields of (A) discharge, (B) biochemical oxygen demand (5-day), and (C) total suspended solids for effluent released from municipal wastewater-treatment facilities in the New Jersey drainage

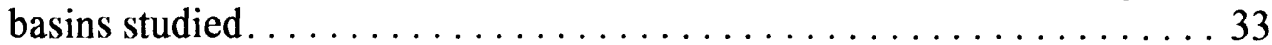

7. Estimated amounts of chloride applied to roadways by the New Jersey Department of Transportation in the New Jersey drainage basins

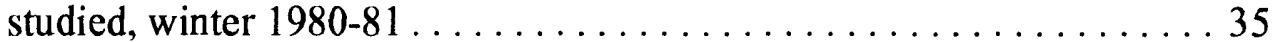

8. Trends in the estimated amounts of sodium and chloride applied to roadways by the New Jersey Department of Transportation in the New Jersey drainage basins studied, winters 1975-76 through 1986-87 . 38

9. Estimated amounts of phosphorus fertilizer applied in the New Jersey drainage basins studied, $1980 \ldots \ldots \ldots \ldots \ldots \ldots \ldots \ldots \ldots 40$

10. Estimated cropland soil-erosion rates in 39 of the New Jersey drainage basins studied, early 1980 's . . . . . . . . . . . . . . . 43

11. Locations of New Jersey stream-monitoring stations and trends in specific conductance, $\mathrm{pH}$, and concentrations of dissolved oxygen, biochemical oxygen demand (5-day), and total nitrogen used in contingency-table analysis ............................... 49

12. Locations of New Jersey stream-monitoring stations and trends in concentrations of total ammonia, total phosphorus, total organic carbon, dissolved calcium, and dissolved magnesium used in contingency-table analysis . .............................. 50

13. Locations of New Jersey stream-monitoring stations and trends in concentrations of dissolved sodium, dissolved potassium, dissolved chloride, fecal coliform bacteria, and fecal streptococcus bacteria used in contingency-table analysis.

14. New Jersey drainage basins having uptrends in $\mathrm{pH}$ and the greatest population density in 1986 of the drainage basins studied, and drainage basins having downtrends in $\mathrm{pH}$ and the least population density in $1986 \ldots 58$

15. New Jersey drainage basins where uptrends in dissolved-oxygen concentrations were found and where greater than 60 percent of effluent discharge originated from nonmunicipal wastewater-treatment facilities . . . . . 60

16. New Jersey drainage basins where uptrends in concentrations of dissolved chloride were found and where the chloride application rate due to road salting were the greatest during winter 1980-81 of the basins studied . . 63

17. New Jersey drainage basins where uptrends in concentrations of fecal streptococcus bacteria were found and where cropland soil-erosion rates ranged from 5.1 to 8.0 tons per acre per year $\ldots \ldots \ldots \ldots \ldots \ldots 66$ 


\section{TABLES}

Page

Table 1. New Jersey drainage basins used to associate trends in water-quality data with basin characteristics . . . . . . . . . . . . . . . . . . . . . 6

2. Results of trend analyses for 13 streamwater constituents and 2 physical properties at selected sites on New Jersey streams, water years 1976-86 . . . . 11

3. Drainage-basin characteristics tested in this study for associations with trends in

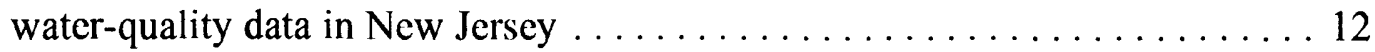

4. Statistical summary of stream-discharge characteristics and trends, water years 1971-91, for New Jersey stream-monitoring stations where continuous discharge measurements and water-quality trend results are available ..... 14

5. Statistical trend results for instantaneous streamflow data, water years 1976-86, at the $60 \mathrm{New}$ Jersey stream-monitoring stations tested for waterquality-data trends. ............................... 16

6. Percentage of land use classified as forested, urban, and agricultural land in the New Jersey drainage basins studied . . . . . . . . . . . . . . . 19

7. Estimated population, population density, and density change from 1975 to 1986 in the New Jersey drainage basins studied . . . . . . . . . . . . . 24

8. Number of, and change in number of, permitted wastewater discharges in New Jersey drainage basins studied, 1975 and $1986 \ldots \ldots \ldots \ldots \ldots \ldots \ldots 28$

9. Permitted effluent discharges in the New Jersey drainage basins studied, 1975 . . 74

10. Permitted effluent discharges and effluent quantity and quality in the New Jersey drainage basins studied, $1986 \ldots \ldots \ldots \ldots \ldots \ldots \ldots \ldots \ldots \ldots$

11. Yields of effluent, biochemical oxygen demand, and total suspended solids from permitted effluent discharges in New Jersey drainage basins studied, and percentage of yields in each drainage basin from municipal treatment facilities.

12. Yields of effluent, biochemical oxygen demand, and total suspended solids from permitted effluent discharges in an area 3.1 miles upstream from the monitoring stations in New Jersey drainage basins studied, and percentage of yields in each drainage basin from municipal treatment facilities . . . . . . . 31

13. Road-salt application in Regions I-IV in New Jersey by winter season, 1975-76

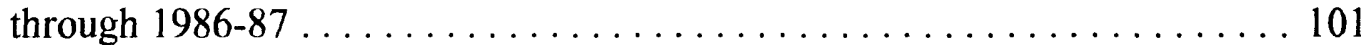

14. Estimated road-salt application in the New Jersey drainage basins studied, in terms of chloride, sodium, and calcium, by basin and winter season,

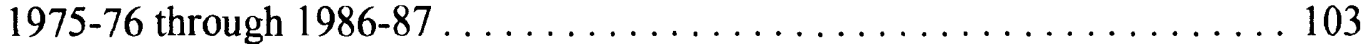

15. Results of statistical testing for trends in the application of chloride, sodium, and calcium to roadways in the New Jersey drainage basins studied,

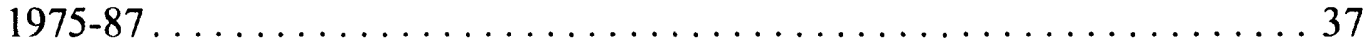

16. Estimated application of phosphorus and nitrogen fertilizer in the New Jersey

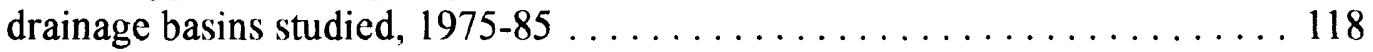

17. Results of statistical testing for trends in the use of phosphorus and nitrogen fertilizers in the New Jersey drainage basins studied, 1975-86 . . . . . . . . 4 


\section{TABLES--Continued}

Table 18. Cropland soil-erosion rates and estimated acreage irrigated in 39 of the New Jersey drainage basins studied, $1986 \ldots \ldots \ldots \ldots \ldots \ldots \ldots \ldots \ldots . \ldots 42$

19. Categorical definitions of drainage-basin characteristics as used in contingencytable analysis of data for the New Jersey drainage basins studied . . . . . . . . 46

20. Probabilities derived from contingency-table analyses of water-quality data and drainage-basin characteristics in the New Jersey drainage basins studied . . . 133

21. Results of Spearman-rho correlation analyses of water-quality data from paired monitoring stations on the same New Jersey stream, water year 1976-86 . . . 139

22. Summary of correlation-test results on water-quality data collected at New Jersey stream-monitoring stations whose trends were used in contingency-

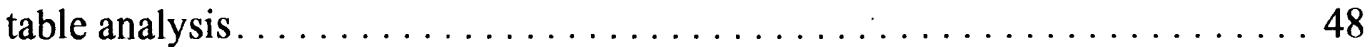

23. Water-quality trend results for New Jersey stream-monitoring stations and streamwater constituents and properties used in contingency-table analysis . 145

24. Streamwater constituents and properties whose trends were significantly associated with drainage-basin characteristics in New Jersey . ......... 52

25. Significant associations among the water-quality trends in the New Jersey drainage basins studied . . . . . . . . . . . . . . . . . . . . 54 


\section{CONVERSION FACTORS}

Multiply

inch (in.)

mile (mi)

square mile $\left(\mathrm{mi}^{2}\right)$

cubic foot per second $\left(\mathrm{ft}^{3} / \mathrm{s}\right)$

million gallons per day (Mgal/d)

million gallons per day per square mile $\left.(\mathrm{Mgal} / \mathrm{d}) / \mathrm{mi}^{2}\right)$

ton, short

ton per square $\mathrm{mi}$ (ton $/ \mathrm{mi}^{2}$ )

kilogram per day $(\mathrm{kg} / \mathrm{d})$

degree Fahrenheit $\left({ }^{\circ} \mathrm{F}\right)$
By

To obtain

Length

25.4

1.609

Area

4,047

2.590

Flow

0.02832

0.04381

0.016911

Mass

0.9072

0.35

Mass Per Unit Time

2.205

pound per day

Temperature

${ }^{\circ} \mathrm{C}=5 / 9 \times\left({ }^{\circ} \mathrm{F}-32\right)$

square meter millimeter

kilometer

square kilometer

cubic meter per second cubic meter per second cubic meter per second per square kilometer

megagram

megagram per square kilometer 


\title{
ASSOCIATIONS BETWEEN WATER-QUALITY TRENDS IN NEW JERSEY STREAMS AND DRAINAGE-BASIN CHARACTERISTICS, 1975-86
}

\author{
By Keith W. Robinson, Timothy R. Lazaro, and Connie Pak
}

\begin{abstract}
Trends in the concentrations of 13 chemical constituents and 2 physical properties measured during 1975-86 at 60 stream-monitoring stations in New Jersey were analyzed for statistical association with drainage-basin characteristics. Basin characteristics assessed for the 60 drainage basins corresponding to the stream-monitoring stations included dominant land use, population, effluent discharge, road salting, fertilizer application, and estimates of soil erosion and irrigated land.

Trends in many constituents and properties were statistically associated $(p \leq 0.10)$ with the dominant land use in the drainage basin, an indication that land use has a strong effect on water-quality trends in New Jersey. Urbanized basins were associated with increasing $\mathrm{pH}$ and increasing concentrations of dissolved sodium, magnesium, and chloride. Upward trends in concentrations of fecal streptococcus bacteria and total ammonia were most common in basins dominated by agricultural land use. Dominant land use nearest the monitoring station tended to be more strongly associated with the water-quality trends than was aggregate land use for the entire basin.

Effluent discharge from wastewater-treatment facilities also appears to have strongly affected water-quality trends in New Jersey drainage basins. Upward trends in $\mathrm{pH}$ and in concentrations of many dissolved ions were commonly found in basins having the greatest amounts of effluent discharged to streams. In addition, upward trends in concentrations of dissolved oxygen were detected in drainage basins where effluent was discharged primarily from nonmunicipal wastewater-treatment facilities rather than from municipal wastewatertreatment facilities. Trends in biochemical oxygen demand and nutrients showed little association with the amount of effluent discharged to streams.
\end{abstract}

Trends in concentrations of some dissolved ions, especially sodium and chloride, were strongly associated with the application rates of road-deicing salts. These associations explain the upward trends in concentrations of dissolved sodium and dissolved chloride commonly found in New Jersey streams. Dissolved-magnesium trends were associated with many drainagebasin characteristics; together these associations indicate storm runoff as a primary contributor of magnesium to streams.

Noteworthy is the absence of statistical associations between the trends in concentrations of nutrients and most measures describing drainage-basin activities. The nearly significant association detected between total phosphorus trends and cropland soil-erosion rates, together with the associations found between total ammonia trends and agricultural land use, seem to indicate that nonpoint sources may be more of an influence on these constituents than effluent discharge. 
No spatial patterns were found in the trends for most water-quality constituents and properties; rather, the trends tended to occur uniformly across the State. An exception was the frequent occurrence of dissolved sodium trends in the northern one-half of New Jersey. In addition, the water-quality trends had little association with the seasons of the year or with changes in streamflow at the time of sampling.

Many of the associations identified in this report match those reported in national studies by previous investigators. However, information describing atmospheric deposition and various other nonpoint sources that have been associated with national water-quality trends were unavailable for the drainage basins discussed in this report.

\section{INTRODUCTION}

Federal and State programs designed to monitor and assess the quality of the Nation's waters increased in the 1970's and 1980's after the enactment of Federal and State clean-water laws. Many of these monitoring programs were intended to assess long-term trends in, and the effects of human activities on, water quality. In New Jersey, routine, statewide water-qualitymonitoring programs have been in place since the mid-1970's.

Also in the 1970's and 1980's, many new Federal and state programs were developed to manage wastes entering surface and ground waters. The control and treatment of wastes to the Nation's rivers, lakes, estuaries, and oceans is estimated to have cost more than $\$ 406$ billion from 1972 through 1986 (U.S. Environmental Protection Agency, 1990a). In New Jersey alone, an estimated $\$ 2.2$ billion in Federal and State grants and loans was spent in this same period for new and upgraded public wastewater-treatment facilities (New Jersey Department of Environmental Protection, 1988). This latter cost for New Jersey does not include monies spent by commercial and industrial wastewater- treatment facilities, nor does it include funds spent by county and municipal governments.

In the 1980 's, some studies were done to assess changes in surface-water quality resulting from water-pollution-control efforts. Smith and others (1987a, 1987b) examined two nationwide monitoring programs for time-series trends in water-quality data, and they attempted to explain the trends in relation to upstream basin characteristics and human activities. In an effort to determine if the regional trends in surface-water-quality data found by Smith and others (1987a, 1987b) could be detected in smaller areas, Hay and Campbell (1990) analyzed surfacewater-quality trends in New Jersey among data collected in water years (WY) $1976-86^{1}$ by the U.S. Geological Survey and the State of New Jersey. Hay and Campbell (1990) detected numerous water-quality trends, but did not evaluate factors that contribute to the occurrence of those trends.

\footnotetext{
${ }^{1}$ In this report, water year refers to the 12-month period October 1 through September 30. The water year is designated by the calendar year in which it ends; thus, the period October 1, 1975, through September 30, 1986, is referred to as "WY 1976-86." All water years are noted "WY"; years not preceded by this notation are calendar years.
} 
The U.S. Geological Survey conducted an investigation during 1989-9I to identify associations between the water-quality trends reported by Hay and Campbell (1990) for New Jersey streams and characteristics of the upstream drainage basins. The purpose of the testing for associations between water- quality trends and basin characteristics is preliminary identification of basin characteristics that may have affected the detected water-quality trends. The results of these tests can then provide information on where more intensive analysis may be done in the future.

Thirteen water-quality constituents and two physical properties that Hay and Campbell (1990) most commonly found to have trends during WY 1976-86 were statistically analyzed to establish associations between trends and basin characteristics. These constituents and properties are $\mathrm{pH}$, specific conductance, dissolved oxygen, biochemical oxygen demand, total ammonia, total nitrogen, total phosphorus, total organic carbon, dissolved calcium, dissolved magnesium, dissolved sodium, dissolved potassium, dissolved chloride, and fecal coliform and fecal streptococcus bacteria. A significant upward trend or downward trend was found for each of these constituents and properties at 10 percent or more of the stream-monitoring stations included in the previous analysis (Hay and Campbell, 1990). Trends in concentrations of two additional constituents, dissolved sulfate and total lead, also were common; however, these constituents were not included because certain laboratory and field- collection methods were found to have produced questionable results during WY 1976-86 (D.A. Rickert, U.S. Geological Survey, written commun., 1989; J.C. Schornick, U.S. Geological Survey, oral commun., 1991).

\section{Purpose and Scope}

This report describes the results of a study to identify statistical associations between the water-quality trends found in New Jersey streams from October 1, 1975, to September 30, 1986, (WY 1976-86) by Hay and Campbell (1990), and characteristics of the drainage basins above the stream-monitoring stations where data were collected. The report also describes the collection and preparation of various data used in the characterization of the drainage basins and the statistical methods employed to associate water-quality trends with the drainage-basin characteristics. Characteristics of basins include type and percentage of various land-use categories, amount and type of treated wastewaters released to streams, population density and change during WY 1976-86, amount of and change in agricultural fertilizer use, severity of soil erosion on cropland, amount of land irrigated for agricultural purposes, and amount of and changes in road-salt use.

\section{Description of the Study Ared}

Sixty drainage basins compose the study area described in this report (fig. 1 and table 1). Seven of the 67 stream-monitoring stations analyzed for water-quality trends by Hay and Campbell (1990) were not included because most of their upstream drainage basin is outside New Jersey. The remaining 60 drainage basins range in size from 1.13 to $490 \mathrm{mi}^{2}$ and represent approximately 40 percent of the total land area of New Jersey. All but two of New Jersey's 21 counties are fully or partly drained by the 60 basins. The drainage basins in this report do not include any tidally influenced waters. 


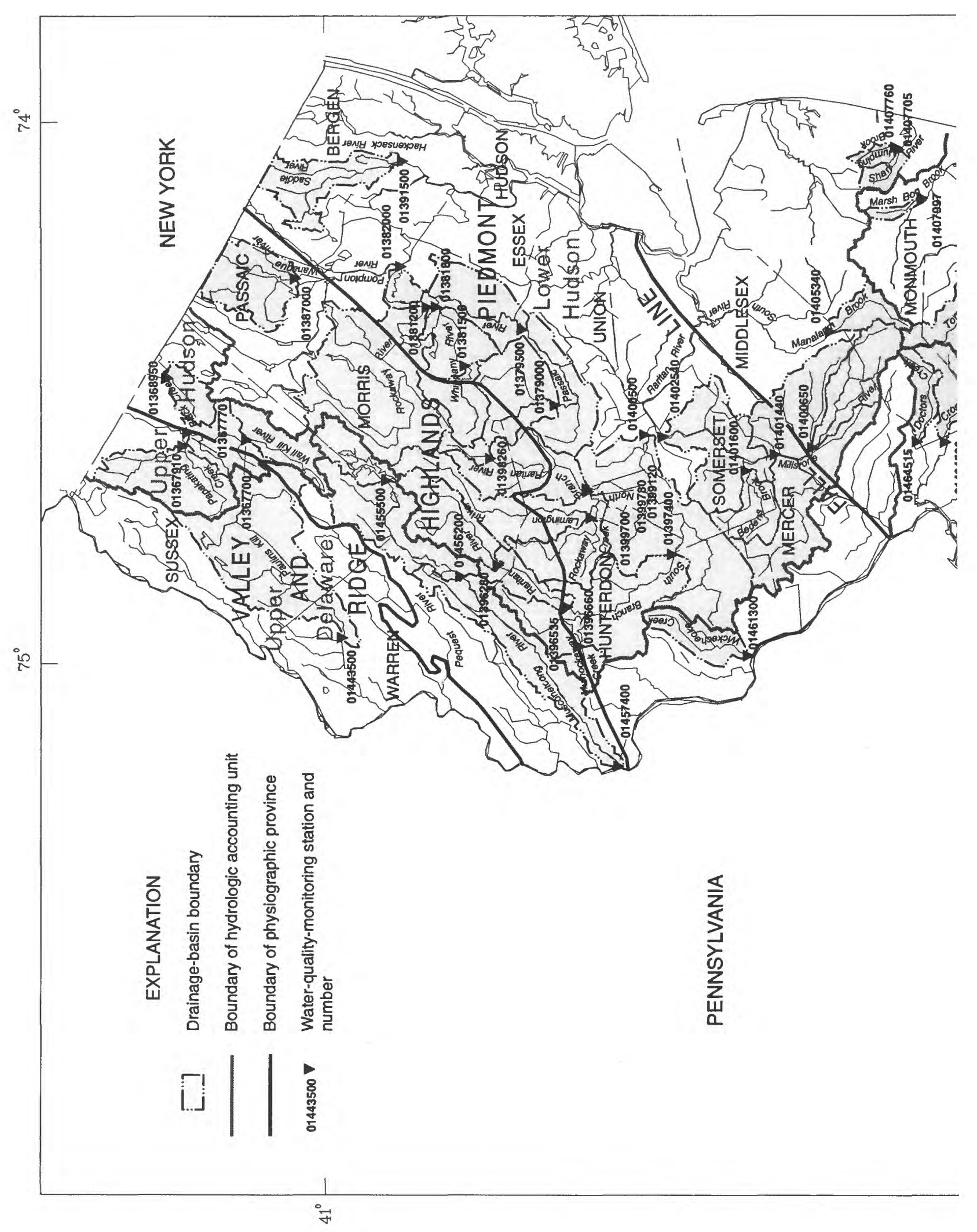




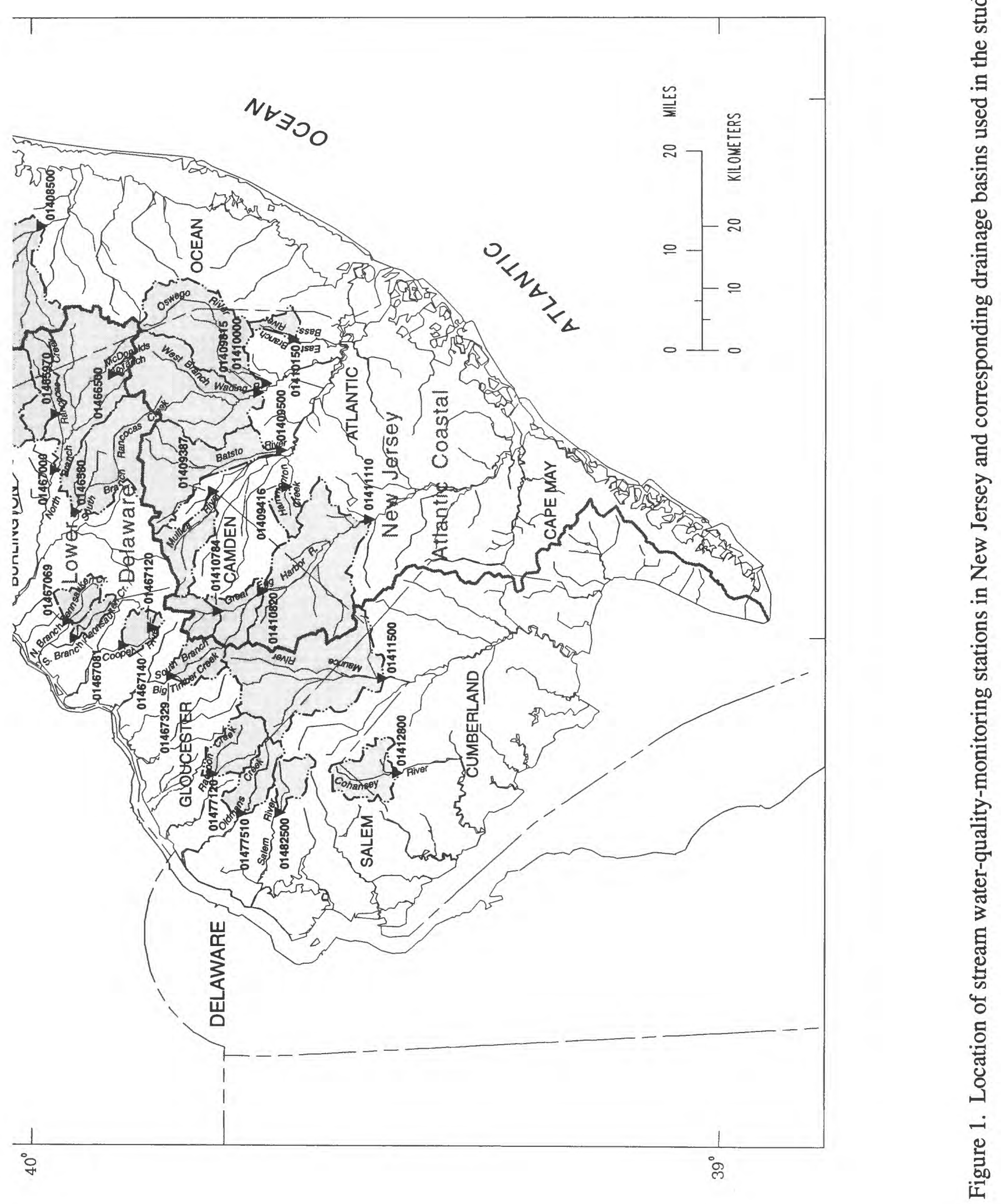


Table 1. New Jersey drainage basins used to associate trends in water-quality data with basin characteristics

$\left[\mathrm{mi}^{2}\right.$, square miles; hydrologic accounting unit from Seaber and others (1987); physiographic province from Ayers and Pustay (1988)]

\begin{tabular}{|c|c|c|c|c|}
\hline Drainage basin & $\begin{array}{c}\text { Monitoring- } \\
\text { station } \\
\text { number }\end{array}$ & $\begin{array}{c}\text { Drainage } \\
\text { area } \\
\left(\mathrm{mi}^{2}\right)\end{array}$ & $\begin{array}{l}\text { Hydrologic } \\
\text { accounting unit }\end{array}$ & $\begin{array}{l}\text { Physiographic } \\
\text { province }\end{array}$ \\
\hline Wallkill River at Franklin & 01367700 & 29.4 & Upper Hudson & Valley and Ridge \\
\hline Wallkill River near Sussex & 01367770 & 60.8 & Upper Hudson & Valley and Ridge \\
\hline Papakating Creek at Sussex & 01367910 & 59.4 & Upper Hudson & Valley and Ridge \\
\hline Black Creek near Vernon & 01368950 & 17.3 & Upper Hudson & Highlands \\
\hline Passaic River near Millington & 01379000 & 55.4 & Lower Hudson & Piedmont \\
\hline Passaic River near Chatham & 01379500 & 100 & Lower Hudson & Piedmont \\
\hline Rockaway River at Pine Brook & 01381200 & 136 & Lower Hudson & Piedmont \\
\hline Whippany River at Morristown & 01381500 & 29.4 & Lower Hudson & Piedmont \\
\hline Whippany River near Pine Brook & 01381800 & 68.5 & Lower Hudson & Piedmont \\
\hline Passaic River at Two Bridges & 01382000 & 361 & Lower Hudson & Piedmont \\
\hline Wanaque River at Wanaque & 01387000 & 90.4 & Lower Hudson & Highlands \\
\hline Saddle River at Lodi & 01391500 & 54.6 & Lower Hudson & Piedmont \\
\hline SB Raritan River at Middle Valley & 01396280 & 47.6 & Lower Hudson & Highlands \\
\hline SB Raritan River at Arch St at High Bridge & 01396535 & 68.8 & Lower Hudson & Piedmont \\
\hline Mulhockaway Creek at Van Syckel & 01396660 & 11.8 & Lower Hudson & Piedmont \\
\hline SB Raritan River at Three Bridges & 01397400 & 181 & Lower Hudson & Piedmont \\
\hline NB Raritan River near Chester & 01398260 & 7.57 & Lower Hudson & Highlands \\
\hline NB Raritan River at Burnt Mills & 01399120 & 63.8 & Lower Hudson & Piedmont \\
\hline Rockaway Creek at Whitehouse & 01399700 & 37.1 & Lower Hudson & Piedmont \\
\hline Lamington (Black) River at Burnt Mills & 01399780 & 100 & Lower Hudson & Piedmont \\
\hline Raritan River at Manville & 01400500 & 490 & Lower Hudson & Piedmont \\
\hline Millstone River at Grovers Mill & 01400650 & 43.4 & Lower Hudson & Piedmont \\
\hline Millstone River at Kingston & 01401440 & 172 & Lower Hudson & Piedmont \\
\hline Beden Brook near Rocky Hill & 01401600 & 27.6 & Lower Hudson & Piedmont \\
\hline Millstone River at Weston & 01402540 & 271 & Lower Hudson & Piedmont \\
\hline Manalapan Brook at Federal Rd near Manalapan & 01405340 & 20.9 & Lower Hudson & Coastal Plain \\
\hline Shark River near Neptune City & 01407705 & 9.96 & Lower Hudson & Coastal Plain \\
\hline Jumping Brook near Neptune City & 01407760 & 6.46 & Lower Hudson & Coastal Plain \\
\hline Marsh Bog Brook at Squankum & 01407997 & 4.91 & N.J. Atlantic Coastal & Coastal Plain \\
\hline Toms River near Toms River & 01408500 & 123 & N.J. Atlantic Coastal & Coastal Plain \\
\hline Mullica River at outlet of Atsion Lake at Atsion & 01409387 & 26.7 & N.J. Atlantic Coastal & Coastal Plain \\
\hline Hammonton Creek at Wescoatville & 01409416 & 9.57 & N.J. Atlantic Coastal & Coastal Plain \\
\hline Batsto River at Batsto & 01409500 & 67.8 & N.J. Atlantic Coastal & Coastal Plain \\
\hline WB Wading River at Maxwell & 01409815 & 85.9 & N.J. Atlantic Coastal & Coastal Plain \\
\hline Oswego River at Harrisville & 01410000 & 72.5 & N.J. Atlantic Coastal & Coastal Plain \\
\hline EB Bass River near New Gretna & 01410150 & 8.11 & N.J. Atlantic Coastal & Coastal Plain \\
\hline Great Egg Harbor River near Sicklerville & 01410784 & 15.1 & N.J. Atlantic Coastal & Coastal Plain \\
\hline Great Egg Harbor River near Blue Anchor & 01410820 & 37.3 & N.J. Atlantic Coastal & Coastal Plain \\
\hline Great Egg Harbor River at Weymouth & 01411110 & 154 & N.J. Atlantic Coastal & Coastal Plain \\
\hline Maurice River at Norma & 01411500 & 112 & Lower Delaware & Coastal Plain \\
\hline Cohansey River at Seeley & 01412800 & 28 & Lower Delaware & Coastal Plain \\
\hline Paulins Kill at Blairstown & 01443500 & 126 & Upper Delaware & Valley and Ridge \\
\hline Musconetcong River at outlet of Lake Hopatcong & 01455500 & 25.3 & Upper Delaware & Highlands \\
\hline Musconetcong River at Beatyestown & 01456200 & 90.3 & Upper Delaware & Highlands \\
\hline Musconetcong River at Riegelsville & 01457400 & 156 & Upper Delaware & Highlands \\
\hline Wickecheoke Creek at Stockton & 01461300 & 26.6 & Upper Delaware & Piedmont \\
\hline Crosswicks Creek at Extonville & 01464500 & 81.5 & Lower Delaware & Coastal Plain \\
\hline Doctors Creek at Allentown & 01464515 & 17.4 & Lower Delaware & Coastal Plain \\
\hline SB Rancocas Creek at Vincentown & 01465850 & 64.5 & Lower Delaware & Coastal Plain \\
\hline NB Rancocas Creek at Browns Mills & 01465970 & 27.4 & Lower Delaware & Coastal Plain \\
\hline McDonalds Branch in Lebanon State Forest & 01466500 & 2.35 & Lower Delaware & Coastal Plain \\
\hline NB Rancocas Creek at Pemberton & 01467000 & 118 & Lower Delaware & Coastal Plain \\
\hline NB Pennsauken Creek near Moorestown & 01467069 & 12.8 & Lower Delaware & Coastal Plain \\
\hline SB Pennsauken Creek at Cherry Hill & 01467081 & 8.98 & Lower Delaware & Coastal Plain \\
\hline Cooper River at Norcross Rd at Lindenwold & 01467120 & 1.13 & Lower Delaware & Coastal Plain \\
\hline Cooper River at Lawnside & 01467140 & 12.7 & Lower Delaware & Coastal Plain \\
\hline SB Big Timber Creek at Blackwood Terrace & 01467329 & 19.1 & Lower Delaware & Coastal Plain \\
\hline Raccoon Creek near Swedesboro & 01477120 & 26.9 & Lower Delaware & Coastal Plain \\
\hline Oldmans Creek at Porches Mill & 01477510 & 21 & Lower Delaware & Coastal Plain \\
\hline Salem River at Woodstown & 01482500 & 14.6 & Lower Delaware & Coastal Plain \\
\hline
\end{tabular}




\section{Physiography, Geology, and Hydrology}

New Jersey encompasses parts of four major physiographic provinces: Valley and Ridge, Highlands, Piedmont, and Coastal Plain (fig. 1) (Ayers and Pustay, 1988). The Coastal Plain and Piedmont provinces are separated by the Fall Line. The topographic, hydrologic, and geologic characteristics of the Coastal Plain are notably different from those of the northern three provinces. The State's surface drainage has been divided into five hydrologic-accounting areas by Seaber and others (1987): Upper Hudson, Lower Hudson, New Jersey Atlantic Coastal, Upper Delaware, and Lower Delaware (fig. 1).

The Coastal Plain is the largest physiographic province in New Jersey, comprising about 55 percent of the State's area. The geology of the Coastal Plain is typically unconsolidated sand, gravel, silt, and clay that thickens south and east of the Fall Line. The materials in the Coastal Plain are primarily marine sediments from the Cretaceous period, and they form extensive aquifers that are the primary source of water for public, commercial, and domestic purposes. Topographically, the Coastal Plain has low relief throughout. As much as 90 percent of the surface water in the Coastal Plain originates from ground-water sources. Ground water and surface water of the Coastal Plain tend to be acidic, have moderate to high noncarbonate hardness, and have low buffering capacity.

North of the Fall Line, the topography changes and land-surface elevation increases with latitude. The Piedmont Province is underlain by shale and sandstone; the Highlands Province by crystalline rocks such as gneiss, marble, and quartzite; and the Valley and Ridge Province by sedimentary units that include limestones. Where glaciation has occurred, glacial sand and gravel have filled the valleys. The quality of ground water and surface water north of the Fall Line is highly variable in quality because of the geologic variety of the three physiographic provinces.

The Upper Hudson drainage in New Jersey, as defined by Seaber and others (1987), includes the Wallkill River and its tributaries. These streams flow north into New York after draining $203 \mathrm{mi}^{2}$ of New Jersey (New Jersey Department of Environmental Protection, 1988). All these streams are in the Valley and Ridge Province. The Lower Hudson drainage includes much of northern and central New Jersey. This area contains the densely populated and industrialized sections of the State that are associated with the New York Metropolitan area. The Raritan River Basin is the largest basin $\left(1,100 \mathrm{mi}^{2}\right)$ that is entirely within the State (New Jersey Department of Environmental Protection, 1988). Major tributaries to the Raritan River are the North Branch Raritan River, the South Branch Raritan River, the Millstone River, and the South River. The Raritan River Basin drains parts of all four physiographic provinces in the State. The Passaic River Basin drains $919 \mathrm{mi}^{2}$ of northeastern New Jersey, including parts of the Piedmont and Highlands Provinces (New Jersey Department of Environmental Protection, 1988). Its major tributaries are the Pompton River, the Rockaway River, and the Saddle River. The Hackensack River Basin, originating in New York, drains $202 \mathrm{mi}^{2}$ of the Piedmont Province in the northeast corner of New Jersey (New Jersey Department of Environmental Protection, 1988). 
The New Jersey Atlantic Coastal drainage includes the eastern half of southern New Jersey. The drainage area is fully within the Coastal Plain Province and consists of many streams that flow east to tidal bays and the Atlantic Ocean. A mixture of population centers, woodlands, and agricultural lands are found in the Atlantic Coastal drainage. Major streams and their drainage basins include the Toms $\left(192 \mathrm{mi}^{2}\right)$, Mullica $\left(569 \mathrm{mi}^{2}\right)$, and Great Egg Harbor $\left(347 \mathrm{mi}^{2}\right.$ ) Rivers (Velnich, 1984).

The western part of New Jersey drains to the Delaware River Basin. The Upper Delaware River drainage area includes all Delaware River drainage from Trenton north and transects all four physiographic provinces in the State. Major tributaries to the Delaware River in this drainage area are Paulins Kill $\left(177 \mathrm{mi}^{2}\right)$, the Pequest River $\left(157 \mathrm{mi}^{2}\right)$, and the Musconetcong River $\left(156 \mathrm{mi}^{2}\right.$ ) (Velnich, 1982). The Lower Delaware drainage area includes all Delaware River and Bay drainage south of Trenton. The Lower Delaware drainage is entirely within the Coastal Plain Province. Major streams in the Lower Delaware include Crosswicks Creek $\left(144 \mathrm{mi}^{2}\right)$, Rancocas Creek $\left(340 \mathrm{mi}^{2}\right)$, the Salem River $\left(117 \mathrm{mi}^{2}\right)$, and the Maurice River $\left(382 \mathrm{mi}^{2}\right)$ (Velnich, 1982). Much of the land use in the Delaware River Basin in New Jersey is farmland and woodland, but major population centers are found at Trenton and Camden.

\section{Climate}

Variation in the distribution of precipitation across the State reflects differences in terrain. Average annual precipitation in New Jersey statewide is 44 in.; annual precipitation ranges from $40 \mathrm{in}$. in the south to $52 \mathrm{in.}$ in the northern mountains (Bauersfeld and others, 1991). Precipitation falls fairly uniformly throughout the year, although the interior of the State tends to receive the greatest amounts in the summer from thunderstorms. Annual snowfall is highly variable, ranging from 13 to $50 \mathrm{in}$. in the extreme southern and northern parts of the State, respectively.

January is typically the coldest month of the year in New Jersey. Average temperatures range from $33^{\circ} \mathrm{F}$ in the south to $25^{\circ} \mathrm{F}$ in the north (Ludlum, 1983). The warmest month usually is July. During July, average temperatures in New Jersey range from $70^{\circ} \mathrm{F}$ in the north to $76^{\circ} \mathrm{F}$ in the southwest.

During 1975-86, precipitation in New Jersey was highly variable in comparison to longterm averages. It was above normal during 1972-75 but well below normal during 1976-77. Lack of precipitation resulted in streamflows during 1976-77 that approached those during the drought of record in the mid-1960's. Precipitation and streamflows recovered in 1978 and 1979 to near and above normal; however, 1980 and 1981 were again years of reduced precipitation and streamflows across the State. A drought emergency was declared for the State from fall 1980 to fall 1981. During 1982-84, precipitation and streamflows were normal to above normal. Reduced precipitation, especially in the southern part of the State, occurred in the final 2 years of the period. 


\section{Previous Investiagtions}

In the 1980's, there was much public and government interest in quantifying improvements in surface-water quality that resulted from national legislation in the 1970's aimed at reducing waste discharges to streams. The Association of State and Interstate Water Pollution Control Administrators (ASIWPCA)(1984) did a nationwide survey of state and interstate water- pollution-control agencies in order to qualitatively identify changes in water quality during 1972-82. Streamwater-quality data and professional opinion were the basis for the conclusions presented in the surveys. After compiling the surveys, ASWIPCA (1984) reported that 13 percent of the stream miles assessed nationwide had improved, whereas 3 percent had declined in quality. Using the same methodology for assessment of New Jersey surface waters, the New Jersey Department of Environmental Protection (NJDEP) (1984) noted a general improvement in water quality statewide as a result of improved wastewater treatment at municipal and industrial wastewater-treatment facilities.

Also in the early 1980's, new applications of statistical methods had been developed that allowed for improved analysis of time-series trends in water-quality data (Hirsch and others, 1982; Hirsch and Slack, 1984; Crawford and others, 1983; van Belle and Hughes, 1984). All these studies promoted the use of the Seasonal Kendall test for detecting trends in water-quality data. This test, a modification of the nonparametric Kendall's tau test, adjusts for the serial correlation of data that results from seasonal variations in water-quality data. Hirsch and others (1982) and Crawford and others (1983) also incorporated regression-analysis techniques for eliminating the detection of water-quality trends resulting from changes in streamflow; this process is termed flow adjustment.

Smith and others $(1987 \mathrm{a}, 1987 \mathrm{~b})$ did trend analysis of water-quality data collected at more than 300 stream-monitoring stations nationwide using the Seasonal Kendall test and flow adjustment. They found widespread increases in concentrations of nitrate, chloride, arsenic, and cadmium; and decreases in concentrations of fecal coliform and fecal streptococcus bacteria and lead from October 1974 through October 1981. Trends in concentrations of suspended solids, phosphorus, and nitrate all exhibited distinct regional patterns.

Smith and others (1987a, 1987b) also identified statistical associations between the detected water-quality trends and various measures of upstream basin characteristics and human activities. They found that improvements in municipal wastewater treatment, declines in leadedgasoline use, increased use of road-deicing materials, increased fertilizer-application rates, and increased combustion of fossil fuels all appear to be associated with the trends of selected constituents and properties. Possible causes of most localized or regional trends could not be determined because of the large scale of their study.

Lettenmaier and others (1991) analyzed water-quality trends at 403 stream-monitoring stations nationwide for 1978-87. The most frequently detected trends included increasing concentrations of dissolved ions and total nitrogen and decreasing concentrations of total phosphorus. Exploratory analysis of possible relations between the trends and drainage-basin characteristics resulted in few significant relations that could explain the observed trends. 
In an effort to determine whether water-quality trends similar to those found by Smith and others (1987a, 1987b) could be detected in New Jersey, Hay and Campbell (1990) did trend analyses of water-quality data from New Jersey streams for WY 1976-86 and 1980-86. Hay and Campbell (1990) described the available water-quality data, the criteria developed to determine if trend analysis was possible, the statistical tests employed, and all trend results. They detected trends at 67 and 86 stream-monitoring stations for WY 1976-86 and 1980-86, respectively. All stations were sampled routinely 4 to 12 times per year for both study periods. The Seasonal Kendall test (flow adjusted, where possible) and the Censored Data Regression method (Cohn and Stedinger, 1987) were used to detect trends in the data for more than 50 constituents and properties.

Trends most frequently identified in New Jersey streams by Hay and Campbell (1990) for the WY 1976-86 and 1980-86 study periods included upward trends in specific conductance and concentrations of fecal streptococcus bacteria and dissolved oxygen, dissolved calcium, dissolved magnesium, dissolved sodium, and dissolved chloride; and upward trends and downward trends in $\mathrm{pH}$ and concentrations of total phosphorous, dissolved sulfate, and fecal coliform bacteria. Downward trends in concentrations of total lead and total organic carbon were noted for WY 1976-86 only. The trend results reported by Hay and Campbell (1990) for the 13 constituents and 2 properties examined in this report are summarized in table 2 . Data from Hay and Campbell (1990) form the basis for associating trends in water quality data with basin characteristics, as discussed in this report.

\section{Acknowledoments}

Various agencies provided the U.S. Geological Survey with much of the information discussed in this report. The authors thank Michael Dillon and Isadore Cooperman of the New Jersey Department of Environmental Protection, Alfred Woodrow of the New Jersey Department of Transportation, Steven Rubin of the U.S. Environmental Protection Agency, and Tom Drewes of the U.S. Natural Resources Conservation Service (formerly the Soil Conservation Service) for their efforts in providing data on basin characteristics.

\section{DRAINAGE-BASIN CHARACTERISTICS}

The water-quality trends in New Jersey streams identified by Hay and Campbell (1990) are inherently related to characteristics of the drainage-basin above the monitoring station. An understanding of drainage-basin characteristics--such as streamflow, land uses, wastewater disposal, and human population--during the period of water-quality data collection used in the trend analysis can assist in determining which basin characteristics may have affected the observed water-quality changes. Other factors such as atmospheric deposition and climatic changes also can contribute to the trends detected in water quality.

Information on selected drainage-basin characteristics, also called ancillary data, that are considered to have important influences on water quality in New Jersey were assembled for the 60 drainage basins analyzed in this report (table 3 ). The ancillary data measure not only static conditions but also change during or preceding the WY 1976-86 study period. The investigators 
Table 2. Results of trend analyses for 13 streamwater constituents and 2 physical properties at selected sites on New Jersey streams, water years $1976-86$

[Based on data from Hay and Campbell (1990). $\mu \mathrm{S} / \mathrm{cm}$, microsiemens per centimeter at 25 degrees Celsius; $\mathrm{mg} / \mathrm{L}$, milligrams per liter; MPN/100 mL, most probable number per 100 milliliters; laboratory codes are those used by the U.S. Geological Survey for data storage and retrieval]

\begin{tabular}{|c|c|c|c|c|}
\hline $\begin{array}{c}\text { Constituent or physical property } \\
\text { (unit) }\end{array}$ & $\begin{array}{l}\text { Laboratory } \\
\text { code }\end{array}$ & $\begin{array}{l}\text { Number of } \\
\text { monitoring } \\
\text { stations } \\
\text { analyzed for } \\
\text { trends }\end{array}$ & $\begin{array}{l}\text { Number } \\
\text { (percent of } \\
\text { total) with an } \\
\text { upward trend }\end{array}$ & $\begin{array}{l}\text { Number } \\
\text { (percent of } \\
\text { total) with a } \\
\text { downward } \\
\text { trend }\end{array}$ \\
\hline Specific conductance $(\mu \mathrm{s} / \mathrm{cm})$ & 00095 & 64 & $22(34)$ & $5(8)$ \\
\hline Dissolved oxygen $(\mathrm{mg} / \mathrm{L})$ & 00300 & 62 & $17(27)$ & $2(3)$ \\
\hline Biochemical oxygen demand, 5 day $(\mathrm{mg} / \mathrm{L})$ & 00310 & 49 & $4(8)$ & $7(14)$ \\
\hline pH (standard units) & 00400 & 61 & $19(31)$ & $6(10)$ \\
\hline Total nitrogen, as $\mathrm{N}(\mathrm{mg} / \mathrm{L})$ & 00600 & 23 & $6(26)$ & $1(4)$ \\
\hline Total ammonia, as N (mg/L) & 00610 & 21 & $9(43)$ & $0(0)$ \\
\hline Total phosphorus, as $\mathrm{P}(\mathrm{mg} / \mathrm{L})$ & 00665 & 46 & $5(11)$ & $4(9)$ \\
\hline Total organic carbon $(\mathrm{mg} / \mathrm{L})$ & 00680 & 57 & $0 \quad(0)$ & $35(61)$ \\
\hline Dissolved calcium (mg/L) & 00915 & 61 & $12(21)$ & $0(0)$ \\
\hline Dissolved magnesium $(\mathrm{mg} / \mathrm{L})$ & 00925 & 61 & $16(26)$ & $3(5)$ \\
\hline Dissolved sodium (mg/L) & 00930 & 62 & $39(63)$ & $3(5)$ \\
\hline Dissolved potassium (mg/L) & 00935 & 62 & $2(3)$ & $17(27)$ \\
\hline Dissolved chloride $(\mathrm{mg} / \mathrm{L})$ & 00940 & 63 & $49(78)$ & $2(3)$ \\
\hline Fecal coliform bacteria (MPN/100 mL) & 31615 & 57 & $8(14)$ & $8(14)$ \\
\hline Fecal streptococcus bacteria (MPN/100 mL) & 31677 & 53 & $17(32)$ & $0(0)$ \\
\hline
\end{tabular}


Table 3. Drainage-basin characteristics tested in this study for associations with trends in water-quality data in New Jersey

$\left[\mathrm{ft}^{3} / \mathrm{s}\right.$, cubic feet per second; $\mathrm{mi}^{2}$, square mile; Mgal $/ \mathrm{d} / \mathrm{mi}^{2}$, million gallons per day per square mile; $\mathrm{kg} / \mathrm{d} /$ $\mathrm{mi}^{2}$, kilograms per day per square mile; ton $/ \mathrm{mi}^{2}$, tons per square mile; ton/acre/yr, tons per acre per year]
Drainage-basin
characteristic (unit)

\section{Description}

Stream discharge

$\left(\mathrm{ft}^{3} / \mathrm{s}\right)$

Drainage basin size

$\left(\mathrm{mi}^{2}\right)$

Physiographic province

Land-cover type (percent of basin)

Population

Wastewater disposal (Mgal/d/mi ${ }^{2}$, $\mathrm{kg} / \mathrm{d} / \mathrm{mi}^{2}$ )

Road-salt application (ton/mi ${ }^{2}$ )

Agricultural fertilizer (ton/mi $\mathrm{m}^{2}$ )

Cropland soil erosion rates/irrigated acreage (ton/acre/yr) (percentage of basin area irrigated)
Annual mean discharge for water years 1971-91 and 1976-86 at continuous gaging stations for 16 drainage basins; trends of either continuous or instantaneous stream discharge for all drainage basins.

Size of the drainage basin above the 60 water-quality monitoring stations.

Physiographic province of the 60 monitoring stations, as listed in table 1.

The percentage of each drainage basin classified as agricultural, forested, or urban land; percentage of these land-cover types also determined for a 3.1- and 0.62-mile upstream area from each monitoring station (Mitchell and others, 1977).

Estimated 1975 and 1986 population for each drainage basin, including change during the period and population density (N.J. Department of Labor, 1976, 1987).

Number of permitted-wastewater treatment facility discharges in each drainage basin for years 1975 and/or 1986; data from the Permit Compliance System data base (U.S. Environmental Protection Agency, 1990b) on the average yields of wastewater discharage and biochemical oxygen demand and total suspended solid loads discharged in 1986 per square mile in the basin and an area 3.1 miles upstream from each monitoring station; and the percentage of wastewater discharge originating from municipal and nonmunicipal facilities.

Estimated amount of calcium, sodium, and chloride applied to roads in each drainage basin during the winter seasons from 1975-76 through 1986-87 by the N.J. Department of Transportation (Alfred Woodrow, N.J. Department of Transportation, written commun., 1989); and trends in calcium, sodium, and chloride use during the same period.

Estimated amount of nitrogen and phosphorus fertilizer used in each basin during 1975-85 for agricultural purposes (Richard Alexander, U.S. Geological Survey, written commun., 1989), and trends in their use during the period.

Average cropland erosion rates and percentage of area irrigated for 39 of the drainage basins (U.S. Department of Agriculture, 1986). 
emphasized collection of ancillary data that were already in digital form for use in a geographic information system (GIS). The GIS was then used to assign all ancillary data to the 60 drainage basins.

Some of the ancillary data are direct measures of contaminant release to streams, such as amounts of effluent (treated wastewater) discharge from wastewater-treatment facilities, whereas other ancillary data are estimators for data that are unavailable. (For example, population density was used as an indicator of the density of urban development.) Some of the ancillary data were available only at the county or the regional level. As a result, the spatial nature of the data can describe a basin characteristic in general terms only. Where the ancillary data were descriptive for an area other than the drainage basin, such as a county or municipality, the data had to be adjusted to represent conditions within the drainage basin.

Certain drainage-basin characteristics and factors thought to be important indicators of water quality in New Jersey were not used in the study because the data were either unavailable or not readily available. These data included information on atmospheric-deposition rates of dissolved materials, the type and intensity of agricultural activities, and the locations of sewered and unsewered areas. Finally, some of the ancillary data described below to characterize drainage basins were not available for WY 1976-86, but rather for years immediately before or after this period.

\section{Streamflow}

Concentrations of many water-quality constituents are strongly related to flows in streams. In addition to the use of flow-adjustment procedures in trends testing by Hay and Campbell (1990) for many constituents, an understanding of streamflow conditions during WY 1976-86 may be helpful in attempting to find causes for the detected water-quality trends.

Streamflow characteristics during WY 1976-86 are available for all 60 drainage basins. Continuous streamflow records were published by the U.S. Geological Survey for 15 of the 60 monitoring stations (table 4). Streamflow was estimated at the remaining 45 water-quality monitoring stations. This estimation of streamflow is based on the observed gage height of the water in the stream at the time of water-quality sample collection; this height is indexed to sites instrumented to record streamflow continuously.

Streamflow characteristics for the 15 continuous-record stations for WY 1976-86 and WY 1972-91, as well as the relative degree to which streamflow in the drainage basin is regulated by upstream diversions and storage, are listed in table 4 . Mean annual streamflow at 14 of the 15 gaging stations for WY 1976-86 was less than the mean annual streamflow during the 21-year period of WY 1972-91. Precipitation during 1975-86 was slightly above normal in the northern part of New Jersey (114 percent of normal at Newark) and below normal in the southern part of the State ( 87 percent of normal at Atlantic City). In addition, the State experienced droughts in 1980-81 and 1985; these droughts are a likely factor in the lower mean annual stream discharges reported for WY 1976-86. 
Table 4. Statistical summary of stream-discharge characteristics and trends, water years 1971-91, for New Jersey stream-monitoring stations where continuous discharge measurements and water-quality trend results are available

[ $\mathrm{ft}^{3} / \mathrm{s}$, cubic feet per second; $\mathrm{n}$, sample size for trends testing and is the number of years of available annual mean streamflows; tau, Kendall's tau computed test statistic; p-value, the probability that the annual mean streamflows during water years 1971-9l resulted from chance rather than an actual change in annual mean flow. Amount of stream discharge regulation in drainage basin from Robert Schopp, U.S. Geological Survey, oral commun., 1993]

\begin{tabular}{|c|c|c|c|c|c|c|c|}
\hline \multirow[b]{2}{*}{ Monitoring-station name } & \multirow{2}{*}{$\begin{array}{l}\text { Monitoring- } \\
\text { station } \\
\text { number }\end{array}$} & \multicolumn{2}{|c|}{$\begin{array}{c}\text { Mean annual } \\
\text { streamflow }\left(\mathrm{ft}^{3} / \mathrm{s}\right) \text {, } \\
\text { for given range of } \\
\text { water years }\end{array}$} & \multirow{2}{*}{$\begin{array}{l}\text { Amount of } \\
\text { stream } \\
\text { discharge } \\
\text { regulation in } \\
\text { drainage } \\
\text { basin }\end{array}$} & \multicolumn{3}{|c|}{$\begin{array}{l}\text { Trends in annual mean flow, } \\
\text { water years 1971-91 }\end{array}$} \\
\hline & & $1971-91$ & $1976-86$ & & $\mathrm{n}$ & tau & p-value \\
\hline Passaic River near Millington & 01379000 & 109 & 102 & Minor & 21 & -0.195 & 0.227 \\
\hline Passaic River near Chatham & 01379500 & 201 & 184 & Minor & 21 & -.229 & .156 \\
\hline Whippany River at Morristown & 01381500 & 66.8 & 62.4 & Minor & 21 & -.171 & .291 \\
\hline Wanaque River at Wanaque & 01387000 & 72.9 & 78.0 & Major & 21 & -.224 & .165 \\
\hline Saddle River at Lodi & 01391500 & 113 & 108 & Moderate & 21 & -.162 & .319 \\
\hline Raritan River at Manville & 01400500 & 922 & 853 & Moderate & 21 & -.162 & .319 \\
\hline Shark River near Neptune City & 01407705 & 15.0 & 14.4 & Moderate & 21 & .010 & .976 \\
\hline Jumping Brook near Neptune City & 01407760 & 10.7 & 10.2 & Moderate & 21 & .086 & .608 \\
\hline Toms River near Toms River & 01408500 & 221 & 212.2 & Minor & 21 & -.210 & .194 \\
\hline Batsto River at Batsto & 01409500 & 120 & 113 & Minor & 21 & -.067 & .695 \\
\hline Crosswicks Creek at Extonville & 01464500 & 147 & 139 & Minor & 21 & -.276 & .085 \\
\hline McDonalds Branch in Lebanon State Forest & 01466500 & 2.24 & 2.13 & None & 21 & -.210 & .194 \\
\hline NB Rancocas Creek at Pemberton & 01467000 & 182 & 171 & Minor & 21 & -.162 & .319 \\
\hline SB Pennsauken Creek at Cherry Hill & 01467081 & 19.2 & 18.2 & Minor & 21 & .474 & .795 \\
\hline Raccoon Creek near Swedesboro & 01477120 & 41.8 & 38.4 & None & 21 & -.219 & .174 \\
\hline
\end{tabular}


Trend analysis of the annual mean streamflow during WY 1972-91 at the 15 continuousrecord stations found no trend at any site for the period $(\alpha=0.05)$. Use of Kendall's tau correlation analysis (Snedecor and Cochran, 1967) showed that at one site, Crosswicks Creek at Extonville (U.S. Geological Survey station number 01464500), a downward trend in annual mean streamflow during the period was significant when $\alpha$ was increased to 0.10 . A number of floods at the beginning of the period may have caused the observed trend in streamflow at this location.

Trends in instantaneous streamflow at the time of water-quality sampling during WY 1976-86 for all 60 stations were determined by use of the Seasonal Kendall test. Significant trends $(\alpha \leq 0.10)$ were found at 12 stations (table 5). Instantaneous discharge decreased at 11 stations; all but two of these sites are located in the southern half of the State (fig. 2). These downward trends could be an artifact of procedural changes in the frequency of water-quality sampling and stream-height measurement that were made during WY 1976-86 at most stations. Sampling frequency was reduced from monthly at the beginning of the period to six times yearly in WY 1981. When the change was made to six samples per year, sample collection was not equally spaced throughout the year but was biased toward low-flow periods.

\section{Geographic Setting}

The geographic setting of a drainage basin is defined as the physiographic province in which the drainage basin is located (fig. 1 and table 1). When a drainage basin spans more than one physiographic province, the province where the monitoring station is located was taken to be the province for the entire basin. One-half of the 60 drainage basins are located entirely or partly in the Coastal Plain Province, which comprises approximately one-half of New Jersey. Nineteen drainage basins (32 percent of the 60) are in the Piedmont Province, four (6 percent) are in the Valley and Ridge Province, and seven (12 percent) are in the Highlands Province. On the whole, the distribution of water-quality monitoring stations and drainage basins by physiographic province is roughly proportional to the areas of the physiographic provinces within the State (fig. 1).

\section{Land Use}

New Jersey is a major industrial and commercial center in the northeastern United States. In 1990, 33 percent of the State was classified as urban land (U.S. Department of Commerce, 1991); no other state in the nation had a higher percentage of urban land. Yet, 21 percent of the land in New Jersey in 1990 was still active farmland. Forests accounted for 25 percent of the land use in the State, and the remaining 21 percent of land consisted of a variety of other uses such as wetlands, transportation corridors, and mining (U.S. Department of Commerce, 1991).

Even though slightly more than one-fifth of New Jersey was farmland in 1990, the amount of farmland has declined steadily throughout the State since the late 1800's. Between 1950 and 1989, the amount of farmland decreased by more than 800,000 acres (New Jersey Department of Agriculture, 1989). Much of this lost farmland was converted to residential, commercial, and industrial uses. 
Table 5. Statistical trend results for instantaneous streamflow data, water years 1976-86, at the 60 New Jersey stream-monitoring stations tested for water-quality-data trends

[ $\mathrm{n}$, sample size for trends testing and is the number of instantaneous streamflow values; tau, Kendell's tau computed test statistic; p-value, the probability that instantaneous streamflow values occur randomly rather than occur as a result in an actual change in streamflow]

\begin{tabular}{|c|c|c|c|c|}
\hline Monitoring-station name & $\begin{array}{c}\text { Monitoring- } \\
\text { station number }\end{array}$ & $\mathbf{n}$ & tau & p-value \\
\hline $\begin{array}{l}\text { Wallkill River at Franklin } \\
\text { Wallkill River near Sussex } \\
\text { Papakating Creek at Sussex } \\
\text { Black Creek near Vernon } \\
\text { Passaic River near Millington }\end{array}$ & $\begin{array}{l}01367700 \\
01367770 \\
01367910 \\
01368950 \\
01379000\end{array}$ & $\begin{array}{l}43 \\
46 \\
43 \\
41 \\
49\end{array}$ & $\begin{array}{r}0.089 \\
-.064 \\
-.096 \\
.066 \\
-.025\end{array}$ & $\begin{array}{r}0.506 \\
.631 \\
.480 \\
.640 \\
.862\end{array}$ \\
\hline $\begin{array}{l}\text { Passaic River near Chatham } \\
\text { Rockaway River at Pine Brook } \\
\text { Whippany River at Morristown } \\
\text { Whippany River near Pine Brook } \\
\text { Passaic River at Two Bridges }\end{array}$ & $\begin{array}{l}01379500 \\
01381200 \\
01381500 \\
01381800 \\
01382000\end{array}$ & $\begin{array}{l}48 \\
52 \\
49 \\
49 \\
50\end{array}$ & $\begin{array}{r}-.107 \\
-.067 \\
-.056 \\
-.005 \\
.073\end{array}$ & $\begin{array}{r}.393 \\
.579 \\
.660 \\
1.000 \\
.544\end{array}$ \\
\hline $\begin{array}{l}\text { Wanaque River at Wanaque } \\
\text { Saddle River at Lodi } \\
\text { SB Raritan River at Middle Valley } \\
\text { SB Raritan River at Arch St at High Bridge } \\
\text { Mulhockaway Creek at Van Syckel }\end{array}$ & $\begin{array}{l}01387000 \\
01391500 \\
01396280 \\
01396535 \\
01396660\end{array}$ & $\begin{array}{l}47 \\
47 \\
41 \\
45 \\
51\end{array}$ & $\begin{array}{l}-.210 \\
-.132 \\
-.101 \\
-.076 \\
-.044\end{array}$ & $\begin{array}{l}.087^{*} \\
.295 \\
.486 \\
.575 \\
.730\end{array}$ \\
\hline $\begin{array}{l}\text { SB Raritan River at Three Bridges } \\
\text { NB Raritan River near Chester } \\
\text { NB Raritan River at Burmt Mills } \\
\text { Rockaway Creek at Whitehouse } \\
\text { Lamington (Black) River at Burnt Mills }\end{array}$ & $\begin{array}{l}01397400 \\
01398260 \\
01399120 \\
01399700 \\
01399780\end{array}$ & $\begin{array}{l}42 \\
46 \\
46 \\
48 \\
40\end{array}$ & $\begin{array}{l}.184 \\
-.036 \\
-.129 \\
-.053 \\
-.082\end{array}$ & $\begin{array}{l}.170 \\
.807 \\
.306 \\
.685 \\
.560\end{array}$ \\
\hline $\begin{array}{l}\text { Raritan River at Manville } \\
\text { Millstone River at Grovers Mill } \\
\text { Millstone River at Kingston } \\
\text { Beden Brook near Rocky Hill } \\
\text { Millstone River at Weston }\end{array}$ & $\begin{array}{l}01400500 \\
01400650 \\
01401440 \\
01401600 \\
01402540\end{array}$ & $\begin{array}{l}51 \\
47 \\
48 \\
52 \\
49\end{array}$ & $\begin{array}{r}.045 \\
.133 \\
.033 \\
-.245 \\
.021\end{array}$ & $\begin{array}{l}.726 \\
.289 \\
.816 \\
.033^{*} \\
.894\end{array}$ \\
\hline $\begin{array}{l}\text { Manalapan Brook at Federal Rd near Manalapan } \\
\text { Shark River near Neptune City } \\
\text { Jumping Brook near Neptune City } \\
\text { Marsh Bog Brook at Squankum } \\
\text { Toms River near Toms River }\end{array}$ & $\begin{array}{l}01405340 \\
01407705 \\
01407760 \\
01407997 \\
01408500\end{array}$ & $\begin{array}{l}37 \\
49 \\
48 \\
44 \\
57\end{array}$ & $\begin{array}{r}-.009 \\
-.031 \\
-.208 \\
-.072 \\
.033\end{array}$ & $\begin{array}{l}1.000 \\
.825 \\
.088^{*} \\
.600 \\
.785\end{array}$ \\
\hline $\begin{array}{l}\text { Mullica River at outlet of Atsion Lake at Atsion } \\
\text { Hammonton Creek at Wescoatville } \\
\text { Batsto River at Batsto } \\
\text { WB Wading River at Maxwell } \\
\text { Oswego River at Harrisville }\end{array}$ & $\begin{array}{l}01409387 \\
01409416 \\
01409500 \\
01409815 \\
014100() 0\end{array}$ & $\begin{array}{l}43 \\
43 \\
50 \\
59 \\
48\end{array}$ & $\begin{array}{r}-.045 \\
.000 \\
-.148 \\
-.218 \\
-.299\end{array}$ & $\begin{array}{l}.759 \\
1.000 \\
.216 \\
.037^{*} \\
.014^{*}\end{array}$ \\
\hline $\begin{array}{l}\text { EB Bass River near New Gretna } \\
\text { Great Egg Harbor River near Sicklerville } \\
\text { Great Egg Harbor River near Blue Anchor } \\
\text { Great Egg Harbor River at Weymouth } \\
\text { Maurice River at Norma }\end{array}$ & $\begin{array}{l}01410150 \\
01410784 \\
01410820 \\
01411110 \\
01411500\end{array}$ & $\begin{array}{l}49 \\
48 \\
53 \\
46 \\
59\end{array}$ & $\begin{array}{r}-.215 \\
-.144 \\
-.056 \\
-.114 \\
.023\end{array}$ & $\begin{array}{l}.074^{*} \\
.243 \\
.644 \\
.375 \\
.852\end{array}$ \\
\hline $\begin{array}{l}\text { Cohansey River at Seeley } \\
\text { Paulins Kill at Blairstown } \\
\text { Musconetcong River at outlet of Lake Hopatcong } \\
\text { Musconetcong River at Beatyestown } \\
\text { Musconetcong River at Riegelsville }\end{array}$ & $\begin{array}{l}01412800 \\
01443500 \\
01455500 \\
01456200 \\
01457400\end{array}$ & $\begin{array}{l}53 \\
51 \\
51 \\
51 \\
44\end{array}$ & $\begin{array}{r}.063 \\
.200 \\
.010 \\
.036 \\
.006\end{array}$ & $\begin{array}{l}.594 \\
.086^{*} \\
.964 \\
.788 \\
1.000\end{array}$ \\
\hline $\begin{array}{l}\text { Wickecheoke Creek at Stockton } \\
\text { Crosswicks Creek at Extonville } \\
\text { Doctors Creek at Allentown } \\
\text { SB Rancocas Creek at Vincentown } \\
\text { NB Rancocas Creek at Browns Mills }\end{array}$ & $\begin{array}{l}01461300 \\
01464500 \\
01464515 \\
01465850 \\
01465970\end{array}$ & $\begin{array}{l}46 \\
48 \\
44 \\
47 \\
47\end{array}$ & $\begin{array}{r}.045 \\
-.144 \\
.042 \\
-.342 \\
-.220\end{array}$ & $\begin{array}{l}.747 \\
.242 \\
.774 \\
.005^{*} \\
.074^{*}\end{array}$ \\
\hline $\begin{array}{l}\text { McDonalds Branch in Lebanon State Forest } \\
\text { NB Rancocas Creek at Pemberton } \\
\text { NB Pennsauken Creek near Moorestown } \\
\text { SB Pennsauken Creek at Cherry Hill } \\
\text { Cooper River at Norcross Rd at Lindenwold }\end{array}$ & $\begin{array}{l}01466500 \\
01467000 \\
01467069 \\
01467081 \\
01467120\end{array}$ & $\begin{array}{l}63 \\
46 \\
49 \\
53 \\
49\end{array}$ & $\begin{array}{l}-0.213 \\
-.317 \\
-.227 \\
-.068 \\
-.130\end{array}$ & $\begin{array}{l}0.032^{*} \\
.011^{*} \\
.058^{*} \\
.565 \\
.285\end{array}$ \\
\hline $\begin{array}{l}\text { Cooper River at Lawnside } \\
\text { SB Big Timber Creek at Blackwood Terrace } \\
\text { Raccoon Creek near Swedesboro } \\
\text { Oldmans Creek at Porches Mill } \\
\text { Salem River at Woodstown }\end{array}$ & $\begin{array}{l}01467140 \\
01467329 \\
01477120 \\
01477510 \\
01482500\end{array}$ & $\begin{array}{l}50 \\
51 \\
50 \\
49 \\
49\end{array}$ & $\begin{array}{r}-.015 \\
.059 \\
.050 \\
-.021 \\
.065\end{array}$ & $\begin{array}{l}.929 \\
.636 \\
.696 \\
.894 \\
.610\end{array}$ \\
\hline
\end{tabular}

* Trend considered significant at $\alpha 0.10$ 


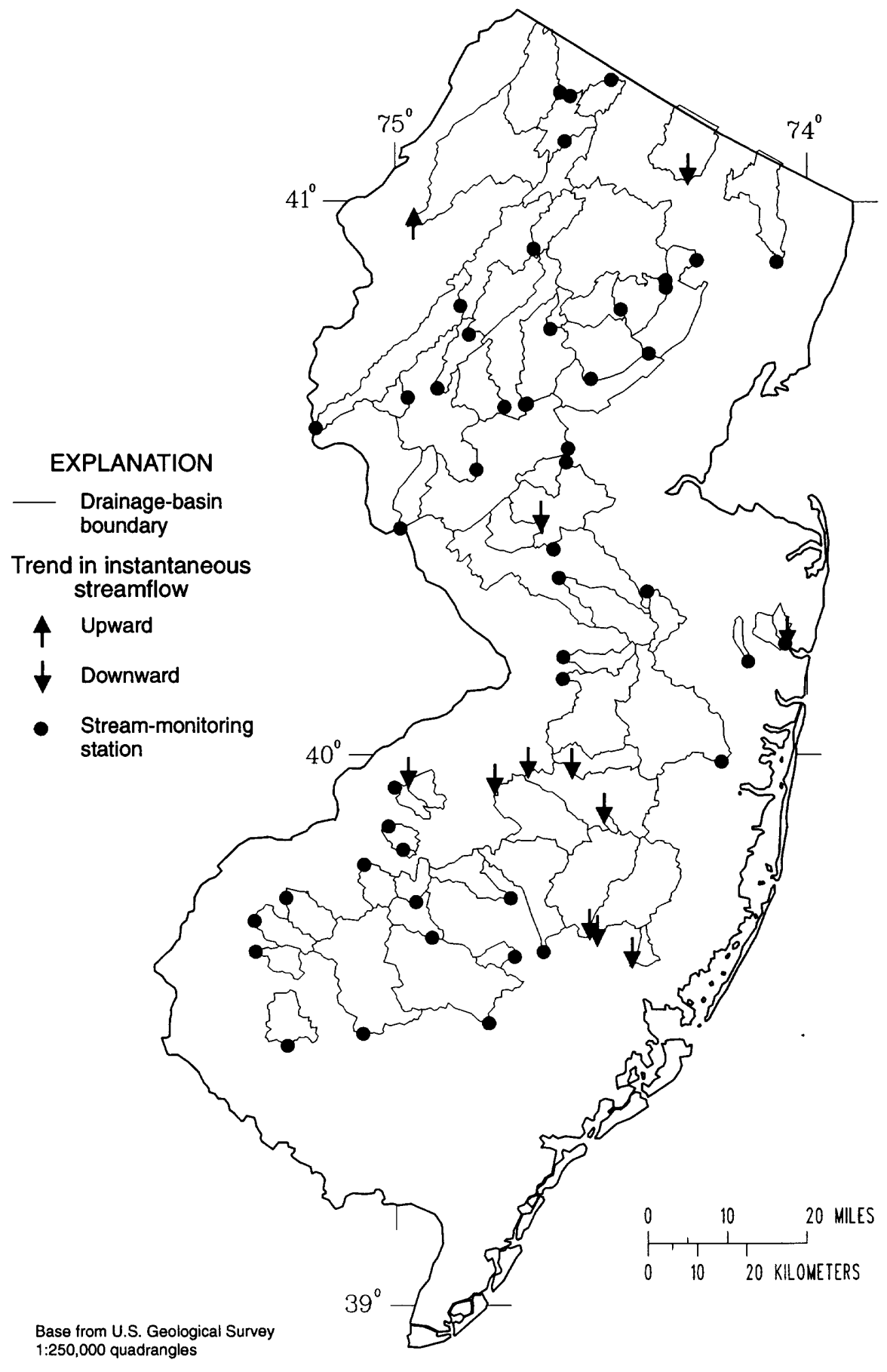

Figure 2. Locations of New Jersey stream-monitoring stations and detected trends in instantaneous streamflow, water years 1976-86. 
Information on the presence and percentages of various land-use types in the 60 drainage basins can help identify the possible effects of land use on the observed water-quality changes. The only data that were readily available for describing land use in each of the 60 drainage basins are data from the National Cartographic Information Center at a scale of 1:250,000 (Mitchell and others, 1977). This national land-use data base, processed through the U.S. Geological Survey's Geographic Information Retrieval and Analysis System (GIRAS), is in digital form and is based on land-cover information from the late 1960's and early 1970's. Depending on the land use category, the GIRAS data base identifies individual land uses to a minimum resolution of approximately 10 acres. Because the GIRAS data have not been updated, no data were available to describe land use within the 60 drainage basins during WY 1976-86.

The GIRAS data differentiate land cover into 9 major classes and 37 subclasses (Anderson and others, 1976). The nine major classes are urban, agriculture, rangeland, forest, water, wetland, barren land, tundra, and perennial snow or ice.

The GIRAS data were assigned to each drainage basin with the assistance of a GIS to determine the type and percentage of each land use within the basin. Because urban, forest, and agriculture are the three most common land uses in the State according to the GIRAS data, the percentage of these land uses was the basis for characterizing each drainage basin (table 6). The percentage of the other six major land-cover types available in GIRAS were not determined because they are either not substantial or not present in the 60 drainage basins. The percentages of urban, agriculture, and forest land in areas $0.62 \mathrm{mi}$ ( 1 kilometer) and $3.1 \mathrm{mi}$ ( 5 kilometers) upstream from each monitoring station (table 6) also were identified with the GIRAS data to determine land use close to the monitoring stations.

Land-use characterization of each drainage basin indicated that forest was the dominant land use in 35 of the 60 drainage basins ( 58 percent); agricultural and urban land uses were dominant in 16 and 9 of the 60 drainage basins ( 27 and 15 percent), respectively (fig. 3). Closer to the monitoring stations, urban and agricultural land uses are more common. In the area $3.1 \mathrm{mi}$ upstream from the monitoring station, forested land use was dominant in 29 of 60 areas (48 percent), agricultural land use was dominant in 19 of 60 areas ( 32 percent), and urban land use was dominant in 12 of 60 areas ( 20 percent). In the area 0.62 mi upstream from the monitoring station, urban and agriculture were each dominant in 22 of the 60 areas ( 37 percent), whereas forest was dominant in 16 of 60 areas ( 27 percent).

The GIRAS land-use data for the 60 drainage basins should be considered only as an indicator of the relative amount of urban, forest, and agricultural land in each basin during WY 1976-86. Because the GIRAS data are based on information that was collected 5 to 10 years before the beginning of WY 1976- 86, the GIRAS data may not accurately portray the amount of land in each land-use category in the drainage basins during the study period. 


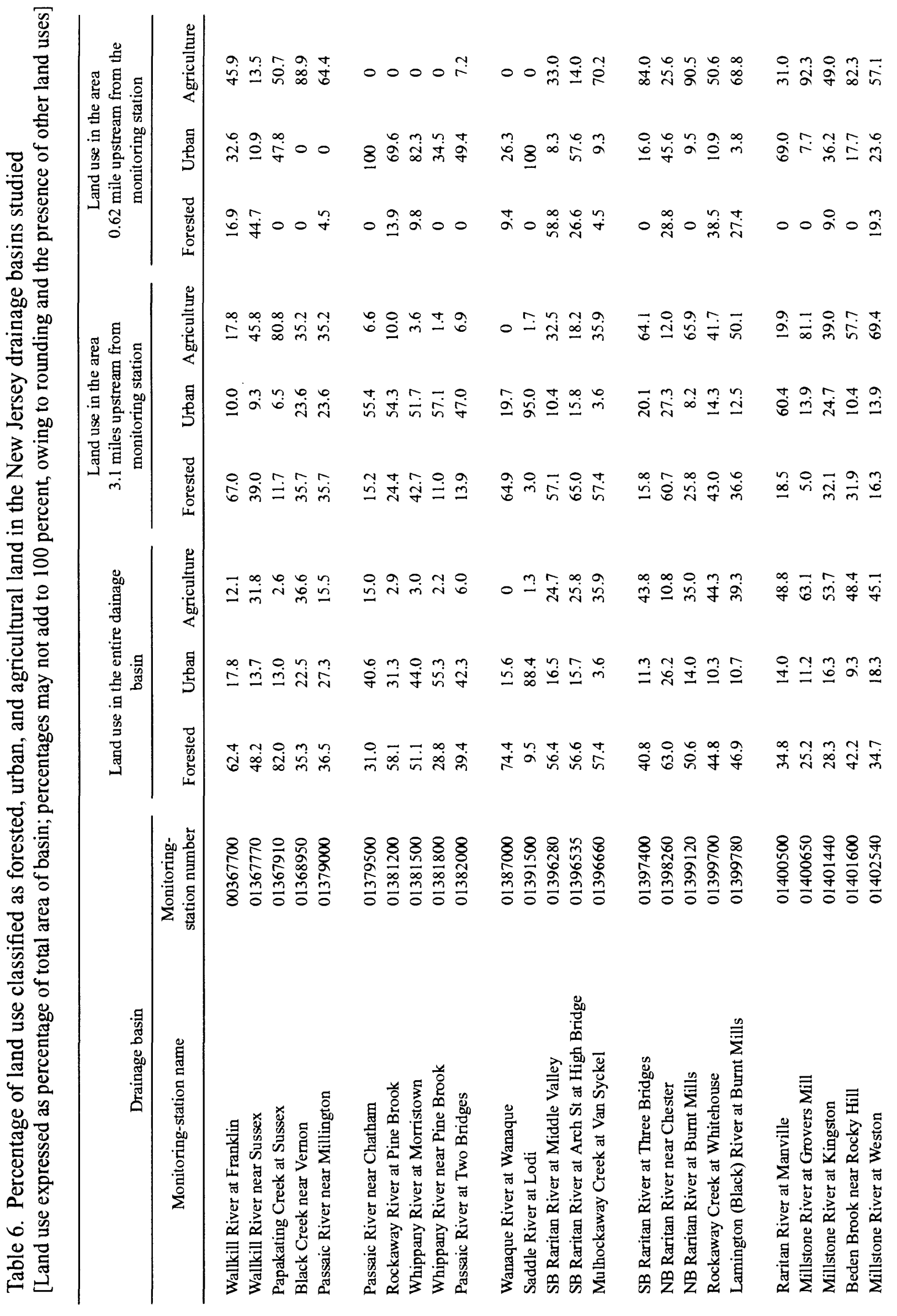




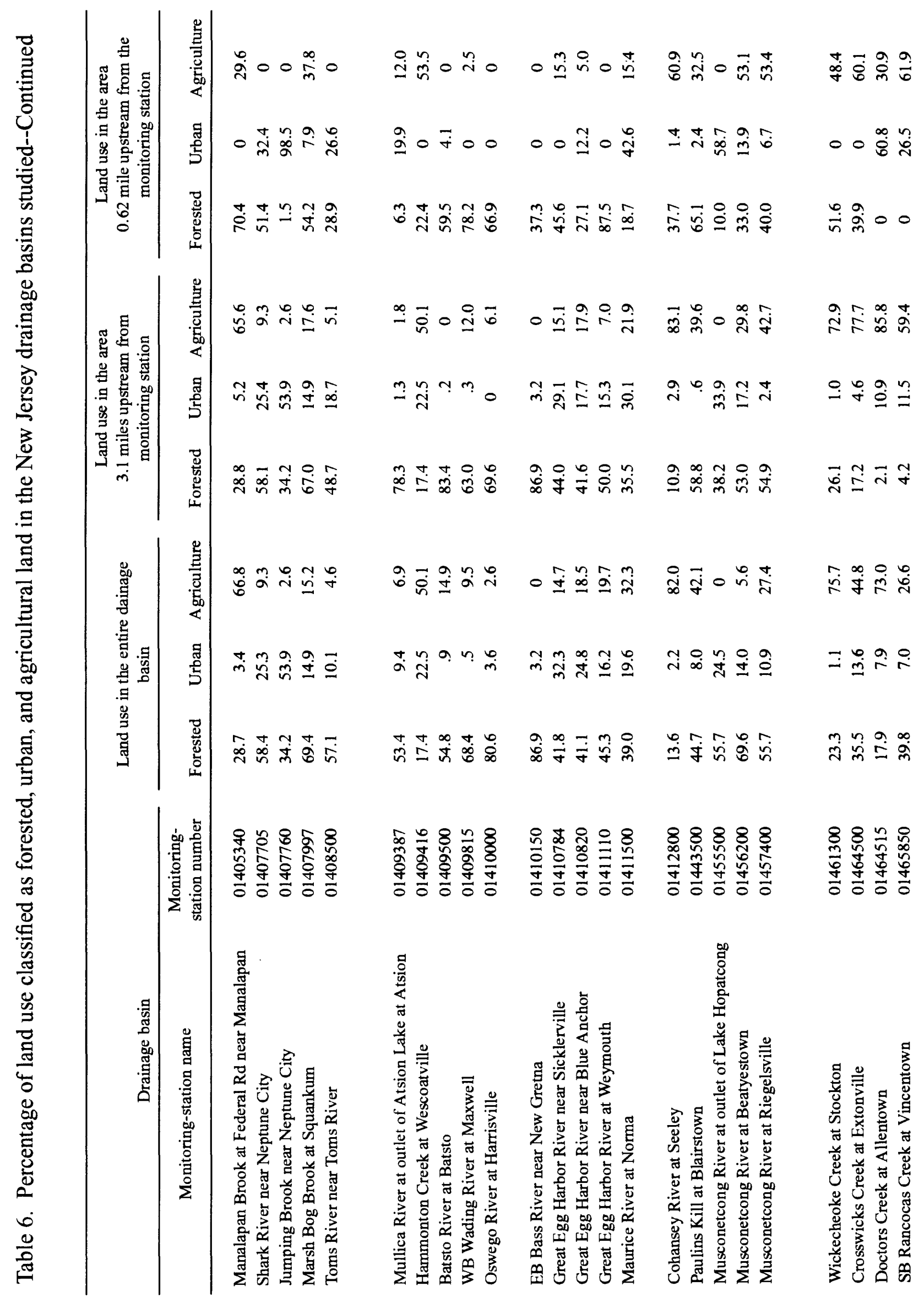




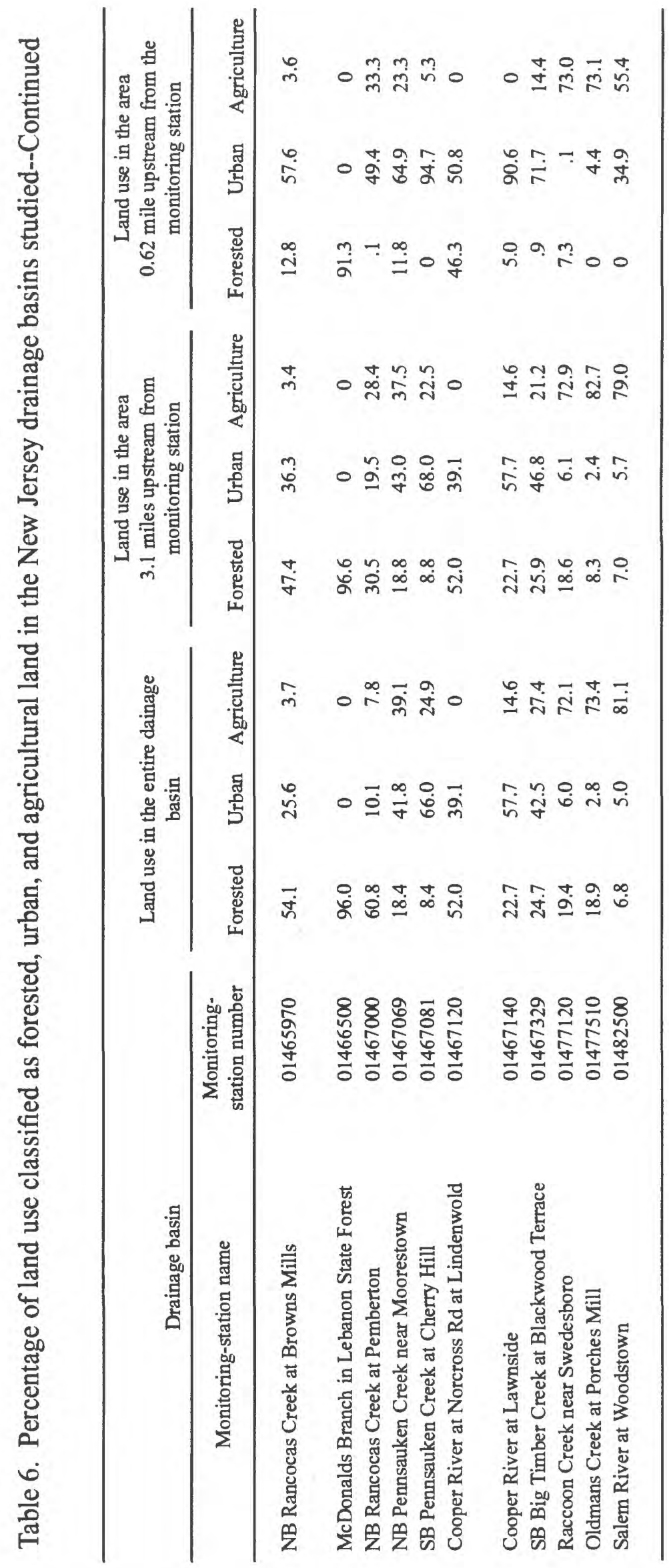



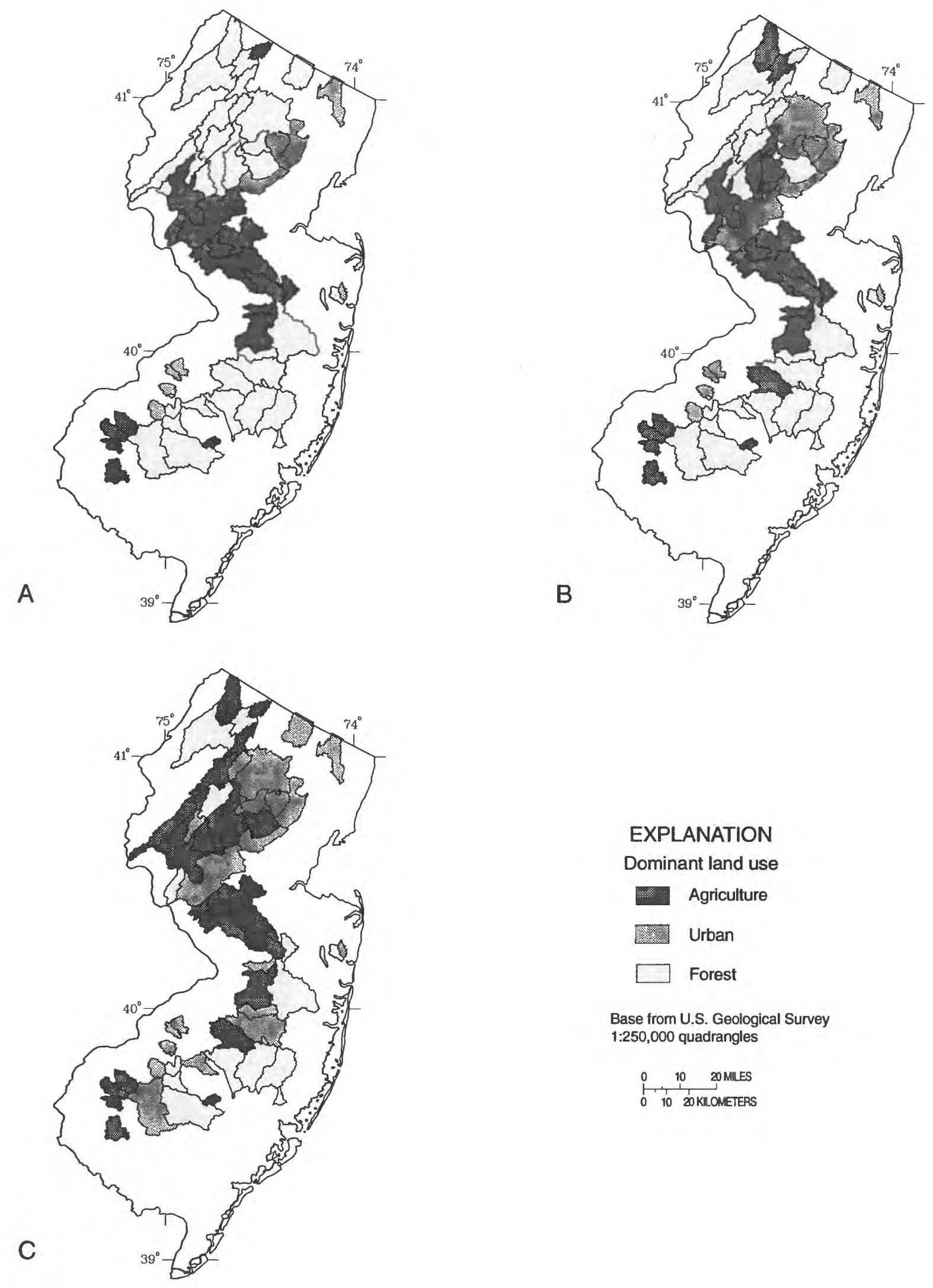

\section{EXPLANATION \\ Dominant land use \\ Agriculture \\ Urban \\ $\square$ Forest}

Base from U.S. Geological Survey

$1: 250,000$ quadrangles

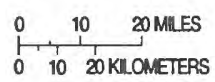

Figure 3. Dominant land use in the 60 New Jersey drainage basins studied: (A) entire drainage basin, (B) area 3.1 miles upstream from the monitoring station, and (C) area 0.62 mile upstream from the monitoring station. (Data from U.S. Geological Survey Geographic Information Retrieval and Analysis System.) 


\section{Population}

New Jersey is the Nation's most densely populated state, having 1,041 persons per square mile in 1990 (Morgan and others, 1992). The State's total population was 7,730,000 in 1990, an increase of nearly 7 percent from the 1970 population of 7,248,000 and an increase of 5 percent from the 1980 population of 7,365,000. Since the 1940 's, population has generally declined in older urban areas and increased in rural and agricultural areas.

The populations of the 60 drainage basins were estimated for 1975 and 1986 by use of information from the New Jersey Department of Labor (NJDOL) $(1976,1987)$ (table 7). The NJDOL population data for these years are municipal-level population estimates based on the previous decennial census. Population densities in 1975 and 1986, and change in density from 1975 to 1986, also were calculated for each drainage basin (table 7). The process of estimating drainage-basin population included the following steps, all done by use of a GIS: (1) conversion of the NJDOL data to population density, expressed as number of persons per square mile for each municipality in a drainage basin, (2) determination of the area of each municipality in a drainage basin, in square miles, (3) multiplication of the municipality's area within a drainage basin by its population density to create an estimated municipal population within the drainage basin, and finally (4) summation of the estimated municipal populations within the drainage basin, expressed as the number of persons.

The greatest number of persons during the study period (418,807 in 1975 and 424,547 in 1986) was in the basin above the Passaic River at Two Bridges. The second greatest population was in the basin above the Raritan River at Manville (165,855 in 1975 and 192,097 in 1986). The greatest population densities were identified in those basins closest to the New York City and Philadelphia metropolitan areas (fig. 4). The greatest population density was in the Cooper River Basin at Lindenwold (2,889 persons per square mile in 1986). The South Branch Big Timber Creek Basin at Blackwood Terrace had the greatest population density increase between 1975 and 1986, an estimated 500 additional persons per square mile over the 11-year period. Population decreases from 1975 to 1986 were identified for two drainage basins, and population in one drainage basin was virtually unchanged. Population increases were recorded in the remaining drainage basins.

\section{Effluent Discharge}

The New Jersey Department of Environmental Protection (1990) reported that the release of effluent from the approximately 1,100 permitted municipal and industrial facilities in the State continues to have an effect on the quality of surface waters, even though decades of extensive water-pollution-control efforts in New Jersey have focused on minimizing the effects of wastewater disposal on surface-water quality. Because of the investment in wastewater treatment and disposal, one would expect that the improvements in wastewater treatment would have a strong effect on the water-quality trends reported by Hay and Campbell (1990).

To determine associations between water-quality trends and effluent discharges, information was collected on the presence of wastewater-treatment facilities in the State and characteristics of the effluent (such as quality and quantity) for WY 1976-86. The primary 
Table 7. Estimated population, population density, and density change from 1975 to 1986 in the New Jersey drainage basins studied [Basin population in number of persons; population density in number of persons per square mile.]

\begin{tabular}{|c|c|c|c|c|c|c|}
\hline \multicolumn{2}{|l|}{ Drainage basin } & \multicolumn{2}{|c|}{1975} & \multicolumn{2}{|c|}{1986} & \multirow{2}{*}{$\begin{array}{c}\text { Change in } \\
\text { population } \\
\text { density, } \\
1975-86\end{array}$} \\
\hline Monitoring-station name & $\begin{array}{l}\text { Monitoring- } \\
\text { station number }\end{array}$ & $\begin{array}{c}\text { Basin } \\
\text { population }\end{array}$ & $\begin{array}{c}\text { Population } \\
\text { density }\end{array}$ & $\begin{array}{c}\text { Basin } \\
\text { population }\end{array}$ & $\begin{array}{c}\text { Population } \\
\text { density }\end{array}$ & \\
\hline Wallkill River at Franklin & 01367700 & 10,999 & 374 & 11,841 & 403 & +29 \\
\hline Wallkill River near Sussex & 01367770 & 19,985 & 329 & 21,227 & 349 & +20 \\
\hline Papakating Creek at Sussex & 01367910 & 7,752 & 131 & 9,481 & 160 & +29 \\
\hline Black Creek near Vernon & 01368950 & 2,672 & 154 & 4,567 & 264 & +110 \\
\hline Passaic River near Millington & 01379000 & 34,943 & 631 & 36.692 & 662 & +31 \\
\hline Passaic River near Chatham & 01379500 & 83,740 & 837 & 85,071 & 851 & +14 \\
\hline Rockaway River at Pine Brook & 01381200 & 131,504 & 967 & 134,956 & 992 & +25 \\
\hline Whippany River at Morristown & 01381500 & 40,339 & 1.372 & 41,203 & 1,401 & +29 \\
\hline Whippany River near Pine Brook & 01381800 & 100,700 & 1,470 & 102,939 & 1,503 & +33 \\
\hline Passaic River at Two Bridges & 01382000 & 418,807 & 1,160 & 424,547 & 1,176 & +16 \\
\hline Wanaque River at Wanaque & 01387000 & 22,408 & 248 & 25,012 & 277 & +29 \\
\hline Saddle River at Lodi & 01391500 & 107,464 & 1,968 & 103,369 & 1,893 & -75 \\
\hline SB Raritan River at Middle Valley & 01396280 & 20,703 & 435 & 25,467 & 535 & +100 \\
\hline SB Raritan River at Arch St at High Bridge & 01396535 & 29,117 & 423 & 35,573 & 517 & +94 \\
\hline Mulhockaway Creek at Van Syckel & 01396660 & 1,513 & 128 & 2,132 & 181 & +53 \\
\hline SB Raritan River at Three Bridges & 01397400 & 53,858 & 298 & 66,560 & 368 & +70 \\
\hline NB Raritan River near Chester & 01398260 & 4,630 & 617 & 5,070 & 676 & +59 \\
\hline NB Raritan River at Burnt Mills & 01399120 & 21,061 & 330 & 23,236 & 364 & +34 \\
\hline Rockaway Creek at Whitehouse & 01399700 & 6,247 & 168 & 8,099 & 218 & +50 \\
\hline Lamington (Black) River at Burnt Mills & 01399780 & 23,199 & 232 & 28,739 & 287 & +55 \\
\hline Raritan River at Manville & 01400500 & 165.855 & 338 & 192,097 & 392 & +54 \\
\hline Millstone River at Grovers Mill & 01400650 & 24,028 & 554 & 26,645 & 614 & +60 \\
\hline Millstone River at Kingston & 01401440 & 90,501 & 526 & 106,333 & 618 & +92 \\
\hline Beden Brook near Rocky Hill & 01401600 & 8,463 & 307 & 9,490 & 344 & +37 \\
\hline Millstone River at Weston & 01402540 & 135,253 & 499 & 159,687 & 589 & +90 \\
\hline Manalapan Brook at Federal Rd near Manalapan & 01405340 & 8,650 & 414 & 12,594 & 603 & +189 \\
\hline Shark River near Neptune City & 01407705 & 6,631 & 670 & 7,410 & 748 & +78 \\
\hline Jumping Brook near Neptune City & 01407760 & 12,325 & 1,926 & 13,028 & 2,036 & +110 \\
\hline Marsh Bog Brook at Squankum & 01407997 & 2,796 & 571 & 3,050 & 622 & +51 \\
\hline Toms River near Toms River & 01408500 & 41,054 & 334 & 52,100 & 424 & +90 \\
\hline Mullica River at outlet of Atsion Lake at Atsion & 01409387 & 6,815 & 255 & 10.538 & 395 & +140 \\
\hline Hammonton Creek at Wescoatville & 01409416 & 2,337 & 246 & 2,452 & 258 & +12 \\
\hline Batsto River at Batsto & 01409500 & 3,381 & 50 & 6,872 & 101 & +51 \\
\hline WB Wading River at Maxwell & 01409815 & 1.999 & 23 & 2,661 & 31 & +8 \\
\hline Oswego River at Harrisville & 01410000 & 3,813 & 53 & 6,895 & 95 & +42 \\
\hline EB Bass River near New Gretna & 01410150 & 148 & 18 & 211 & 26 & +8 \\
\hline Great Egg Harbor River near Sicklerville & 01410784 & 14,001 & 927 & 16,997 & 1,126 & +198 \\
\hline Great Egg Harbor River near Blue Anchor & 01410820 & 21,643 & 580 & 27,311 & 732 & +152 \\
\hline Great Egg Harbor River at Weymouth & 01411110 & 49.812 & 323 & 62,009 & 403 & +80 \\
\hline Maurice River at Norma & 01411500 & 42,233 & 377 & 47,468 & 424 & +47 \\
\hline Cohansey River at Seeley & 01412800 & 4,662 & 167 & 4,973 & 178 & +11 \\
\hline Paulins Kill at Blairstown & 01443500 & 23,158 & 184 & 27,297 & 217 & +33 \\
\hline Musconetcong River at outlet of Lake Hopatcong & 01455500 & 15,552 & 615 & 17,313 & 684 & +69 \\
\hline Musconetcong River at Beatyestown & 01456200 & 53,285 & 590 & 59,366 & 657 & +67 \\
\hline Musconetcong River at Riegelsville & 01457400 & 69,836 & 448 & 78,063 & 500 & +52 \\
\hline Wickecheoke Creek at Stockton & 01461300 & 3,069 & 115 & 3,776 & 142 & +27 \\
\hline Crosswicks Creek at Extonville & 01464500 & 22,734 & 279 & 22,855 & 280 & +1 \\
\hline Doctors Creek at Allentown & 01464515 & 2.447 & 141 & 2,551 & 147 & +6 \\
\hline SB Rancocas Creek at Vincentown & 01465850 & 9,901 & 154 & 11,284 & 175 & +21 \\
\hline NB Rancocas Creek at Browns Mills & 01465970 & 10.547 & 385 & 11.641 & 425 & +40 \\
\hline McDonalds Branch in Lebanon State Forest & 01466500 & 54 & 23 & 48 & 21 & -2 \\
\hline NB Rancocas Creek at Pemberton & 01467000 & 32,072 & 272 & 42,236 & 358 & +86 \\
\hline NB Pennsauken Creek near Moorestown & 01467069 & 10,033 & 784 & 14,366 & 1,122 & +338 \\
\hline SB Pennsauken Creek at Cherry Hill & 01467081 & 17,878 & 2,009 & 19,002 & 2,135 & +126 \\
\hline Cooper River at Norcross Rd at Lindenwold & 01467120 & 3,114 & 2,831 & 3,178 & 2,889 & +58 \\
\hline Cooper River at Lawnside & 01467140 & 26.398 & 2.079 & 29,936 & 2,357 & +279 \\
\hline SB Big Timber Creek at Blackwood Terrace & 01467329 & 22,178 & 1.161 & 30,551 & 1.600 & +439 \\
\hline Raccoon Creek near Swedesboro & 01477120 & 4,960 & 184 & 5,984 & 222 & +38 \\
\hline Oldmans Creek at Porches Mill & 01477510 & 1.727 & 82 & 1,993 & 95 & +13 \\
\hline Salem River at Woodstown & 01482500 & 1.828 & 125 & 1,846 & 126 & +1 \\
\hline
\end{tabular}




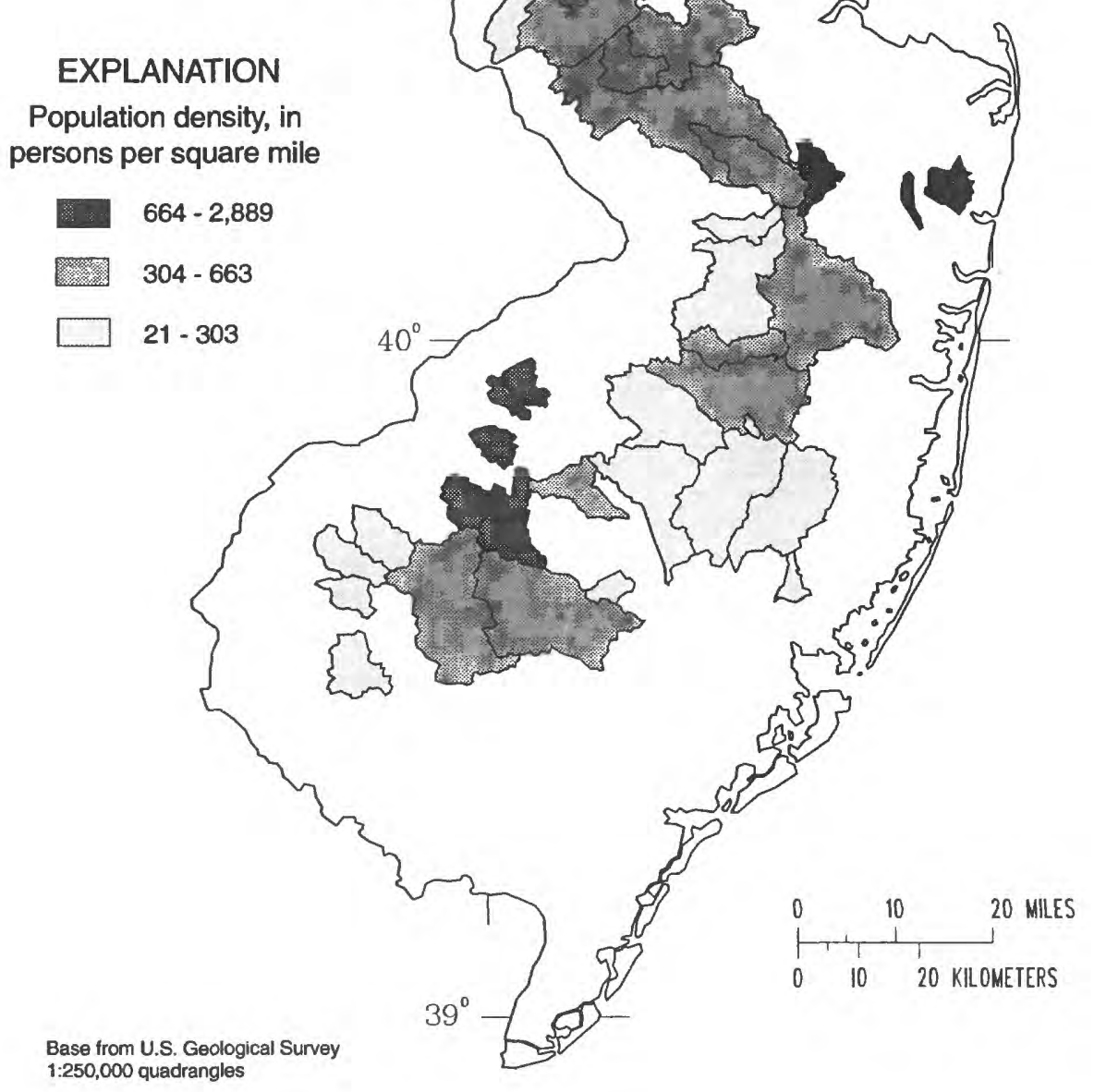

Figure 4. Estimated 1986 population density in the New Jersey drainage basins studied. 
source of this information was the national Permit Compliance System (PCS) data base of the U.S. Environmental Protection Agency (1990b). The computerized PCS data base is maintained in conjunction with a national permitting program designed to control the treatment and release of wastewaters. These permits, known as National Pollutant Discharge Elimination System (NPDES) permits, are issued by the NJDEP (New Jersey Department of Environmental Protection) in New Jersey. As a result, the permits are called New Jersey Pollutant Discharge Elimination System (NJPDES) permits.

The PCS data base contains a variety of information that describes effluent discharges in New Jersey from the mid-1970's to the present. PCS data obtained for this study included data on the location of effluent discharges, the type of facility producing the effluent (either municipal or nonmunicipal), and the quantity and quality of the effluent. However, PCS data on effluent quality and quantity in New Jersey are generally not considered reliable or complete prior to the mid-1980's (Isadore Cooperman, New Jersey Department of Environmental Protection, oral commun., 1989). Because the PCS data base may not be reliable and complete for characterizing effluent released during much of the study period, a number of data-collection and -verification processes had to be used to build a data base on effluent discharges in the 60 drainage basins. These processes included (1) searching for and collecting data on effluentdischarge locations and on effluent quantity and quality from numerous sources, (2) verification of effluent-discharge locations by comparing location information from multiple sources, and (3) checking effluent-quantity and -quality data against published and other, more recent data. A thorough description of these data-collection and -verification processes can be found in Robinson and others (1995).

Digital data on the location, type, quantity, and quality of permitted effluent discharges in New Jersey for 1975 and 1986 were obtained from the PCS data base with the assistance of the NJDEP. Data for some wastewater facilities were incomplete for 1986; supplementary data for those facilities were obtained from the USEPA and by a review of paper files at the offices of the NJDEP in Trenton, N.J. PCS data from 1989 also were used to supplement missing PCS data from earlier years.

On the basis of the latitude and longitude data given in PCS, 1,380 and 1,226 permitted effluent discharges were identified in New Jersey for 1975 and 1986, respectively. To verify the location information, study personnel compared PCS latitude and longitude data with other locational information in PCS for the discharge facility (such as address, county, and known receiving water of the discharge). Where entries were found to be missing or incorrect, other information sources, such as The Environmental Information Inventory. Division of Water Resources (New Jersey Department of Environmental Protection, 1985) and paper files at the offices of the NJDEP in Trenton, N.J., were used to identify the correct locations.

Of the 1,380 permitted effluent discharges in New Jersey in 1975, 263 (19 percent) were in 49 of the 60 drainage basins studied (no permitted effluent discharges were on record for 11 drainage basins). In 1986, 46 drainage basins contained 296 ( 24 percent) of the total 1,226 permitted effluent discharges in the State. The number of wastewater discharges in each 
drainage basin for 1975 and 1986 are listed in table 8 . A complete listing of the years 1975 and 1986 permitted effluent discharges is given by drainage basin in tables 9 and 10 , respectively (at back of the report).

The greatest number of permitted effluent discharges, 85 in 1975 and 88 in 1986, were in the drainage basin above the Passaic River at Two Bridges. This drainage basin is in the heavily developed northeastern part of New Jersey that is adjacent to the New York City metropolitan area. The drainage basin above the Raritan River at Manville contained 59 permitted effluent discharges in 1986. (The Raritan River drains the industrialized central part of New Jersey.) The largest increase in the number of effluent discharges during 1975-86--an increase of 10--was in the Millstone River drainage basin above the Weston monitoring station. In contrast, decreases in the number of permitted effluent discharges were recorded for seven drainage basins during the 11-year period.

Data in PCS describing the quantity and quality of effluent discharged in 1975 and 1986 for each permitted facility in the drainage basins were provided by the NJDEP (Michael Dillon, New Jersey Department of Environmental Protection, written commun., 1990). The volume and rate of effluent discharged by a facility is most frequently stored in PCS in units of million gallons per day. Effluent quality is commonly reported as milligrams per liter of 5-day biochemical oxygen demand (BOD) and total suspended solids (TSS). Discharges of BOD and TSS were characterized for this study in units of kilograms per day. BOD and TSS loads were collected or calculated in kilograms per day because this was the unit of measurement most commonly used by PCS for these constituents.

The PCS data base contained data on the quantity and quality of effluent discharged in 1975 for only about 20 percent of the 263 permitted-wastewater discharges. Because PCS was the only available data base containing information on effluent discharged in 1975 for the entire State, no additional attempts were made to gather the missing 1975 data. As a result, neither the quantity and quality of effluent released in 1975 nor the changes in effluent quality and quantity during the trends-study period of WY 1976-86 could be evaluated.

Effluent quantity and quality data for 1986 were available in PCS for 46 percent (136) of the 296 permitted effluent discharges in the 60 drainage basins. To fill the gaps in 1986 data, study personnel used PCS data for 1989 because the 1989 data were more complete than previous years' data (Isadore Cooperman, New Jersey Department of Environmental Protection, oral commun., 1990). The 1989 PCS data were assumed to be representative of effluent quantity and quality for 1986. An additional 104 permitted discharges (35 percent of the total) were characterized by use of the 1989 PCS data (Steve Rubin, U.S. Environmental Protection Agency, written commun., 1991). Quantity and quality data for the remaining 19 percent (56 discharges) were gathered from paper files at the offices of the NJDEP in Trenton, N.J.

Median effluent flow in million gallons per day, and loads of BOD and TSS discharged in kilograms per day were generated for each discharge from the collected data. If loads of BOD and TSS were present in PCS, then they were used directly. If only BOD and TSS concentrations were reported, then BOD and TSS loads were calculated from reported 
Table 8. Number of, and change in number of, permitted wastewater discharges in New Jersey drainage basins studied, 1975 and 1986

\begin{tabular}{|c|c|c|c|c|}
\hline \multicolumn{2}{|l|}{ Drainage basin } & \multicolumn{2}{|c|}{ Number of permitted discharges } & \multirow{2}{*}{$\begin{array}{c}\text { Change in } \\
\text { number, } 1975 \text { to } \\
1986\end{array}$} \\
\hline Monitoring-station name & $\begin{array}{c}\text { Monitoring-station } \\
\text { number }\end{array}$ & 1975 & 1986 & \\
\hline Wallkill River at Franklin & 01367700 & 2 & 5 & +3 \\
\hline Wallkill River near Sussex & 01367770 & 9 & 11 & +2 \\
\hline Papakating Creek at Sussex & 01367910 & 1 & 3 & +2 \\
\hline Black Creek near Vernon & 01368950 & 3 & 4 & +1 \\
\hline Passaic River near Millington & 01379000 & 2 & 2 & 0 \\
\hline Passaic River near Chatham & 01379500 & 16 & 15 & -1 \\
\hline Rockaway River at Pine Brook & 01381200 & 27 & 32 & +5 \\
\hline Whippany River at Morristown & 01381500 & 7 & 7 & 0 \\
\hline Whippany River near Pine Brook & 01381800 & 20 & 23 & +3 \\
\hline Passaic River at Two Bridges & 01382000 & 85 & 88 & +3 \\
\hline Wanaque River at Wanaque & 01387000 & 2 & 4 & +2 \\
\hline Saddle River at Lodi & 01391500 & 8 & 9 & +1 \\
\hline SB Raritan River at Middle Valley & 01396280 & 6 & 7 & +1 \\
\hline SB Raritan River at Arch St at High Bridge & 01396535 & 6 & 7 & +1 \\
\hline Mulhockaway Creek at Van Syckel & 01396660 & 1 & 1 & 0 \\
\hline SB Raritan River at Three Bridges & 01397400 & 16 & 21 & +5 \\
\hline NB Raritan River near Chester & 01398260 & 1 & 1 & 0 \\
\hline NB Raritan River at Bumt Mills & 01399120 & 9 & 9 & 0 \\
\hline Rockaway Creek at Whitehouse & 01399700 & 3 & 4 & +1 \\
\hline Lamington (Black) River at Burnt Mills & 01399780 & 10 & 9 & -1 \\
\hline Raritan River at Manville & 01400500 & 54 & 59 & +5 \\
\hline Millstone River at Grovers Mill & 01400650 & 4 & 6 & +2 \\
\hline Millstone River at Kingston & 01401440 & 21 & 30 & +9 \\
\hline Beden Brook near Rocky Hill & 01401600 & 2 & 3 & +1 \\
\hline Millstone River at Weston & 01402540 & 36 & 46 & +10 \\
\hline Manalapan Brook at Federal Rd near Manalapan & 01405340 & 0 & 1 & +1 \\
\hline Shark River near Neptune City & 01407705 & 1 & 2 & +1 \\
\hline Jumping Brook near Neptune City & 01407760 & 1 & 0 & -1 \\
\hline Marsh Bog Brook at Squankum & 01407997 & 2 & 0 & -2 \\
\hline Toms River near Toms River & 01408500 & 3 & 4 & +1 \\
\hline Mullica River at outlet of Atsion Lake at Atsion & 01409387 & 0 & 0 & 0 \\
\hline Hammonton Creek at Wescoatville & 01409416 & 2 & 2 & 0 \\
\hline Batsto River at Batsto & 01409500 & 0 & 0 & 0 \\
\hline WB Wading River at Maxwell & 01409815 & 0 & 0 & 0 \\
\hline Oswego River at Harrisville & 01410000 & 0 & 0 & 0 \\
\hline EB Bass River near New Gretna & 01410150 & 0 & 0 & 0 \\
\hline Great Egg Harbor River near Sicklerville & 01410784 & 1 & 1 & 0 \\
\hline Great Egg Harbor River near Blue Anchor & 01410820 & i & i & 0 \\
\hline Great Egg Harbor River at Weymouth & 01411110 & 4 & 2 & -2 \\
\hline Maurice River at Norma & 01411500 & 6 & 5 & -1 \\
\hline Cohansey River at Seeley & 01412800 & 1 & 1 & 0 \\
\hline Paulins Kill at Blairstown & 01443500 & 7 & 12 & +5 \\
\hline Musconetcong River at outlet of Lake Hopatcong & 01455500 & 3 & 4 & +1 \\
\hline Musconetcong River at Beatyestown & 01456200 & 7 & 9 & +2 \\
\hline Musconetcong River at Riegelsville & 01457400 & 12 & 17 & +5 \\
\hline Wickecheoke Creek at Stockton & 01461300 & 2 & 2 & 0 \\
\hline Crosswicks Creek at Extonville & 01464500 & 6 & 6 & 0 \\
\hline Doctors Creek at Allentown & 01464515 & 1 & 1 & 0 \\
\hline SB Rancocas Creek at Vincentown & 01465850 & 3 & 3 & 0 \\
\hline NB Rancocas Creek at Browns Mills & 01465970 & 0 & 0 & 0 \\
\hline McDonalds Branch in Lebanon State Forest & 01466500 & 0 & 0 & 0 \\
\hline NB Rancocas Creek at Pemberton & 01467000 & 2 & 0 & -2 \\
\hline NB Pennsauken Creek near Mooreslown & 01467069 & 1 & 1 & 0 \\
\hline SB Pennsauken Creek at Cherry Hill & 01467081 & 2 & 3 & +1 \\
\hline Cooper River at Norcross Rd at Lindenwold & 01467120 & 0 & 0 & 0 \\
\hline Cooper River at Lawnside & 01467140 & 7 & 8 & +1 \\
\hline SB Big Timber Creek at Blackwood Terrace & 01467329 & 1 & 1 & 0 \\
\hline Raccoon Creek near Swedesboro & 01477120 & i & 1 & 0 \\
\hline Oldmans Creek at Porches Mill & 01477510 & 0 & 0 & 0 \\
\hline Salem River at Woodstown & 01482500 & 0 & 0 & 0 \\
\hline
\end{tabular}


concentration data and effluent-discharge flow data. The median flow and the loads of BOD and TSS compiled for each permitted effluent discharge in 1986 are listed in table 10 (at back of the report).

The data on the quantity and quality of effluent for each wastewater facility were summarized into measures of effluent yields for each drainage basin. These yields were designed to provide a comparative assessment of the amount of effluent and BOD and TSS discharged in the 60 drainage basins. Yields were calculated by summing all effluent flows and loads of BOD and TSS in a drainage basin, and then dividing by the total drainage-basin area. Yields of effluent flows are in units of million gallons per day per square mile discharged, and for BOD and TSS as kilograms per day per square mile (table 11). In addition, effluent yields for the area $3.1 \mathrm{mi}$ upstream from each monitoring station also were estimated (table 12) to determine whether effluent discharges and loads were greatest near the monitoring station or further upstream. Of the 60 drainage basins, 36 contained a permitted effluent discharge in the area 3.1 mi upstream from the monitoring station.

The greatest yields of effluent discharge and BOD released were in the drainage basin of the Cooper River at Lawnside; the Cooper River drains a heavily developed area to the Delaware River in southwestern New Jersey adjacent to Philadelphia. Overall, drainage basins in the central and northeastern parts of the State tended to have greater yields of effluent discharge and BOD and TSS than did other areas of the State (fig. 5). The median yields of effluent discharge and BOD and TSS released in the drainage basins were $0.03 \mathrm{Mgal} / \mathrm{d} / \mathrm{mi}^{2}$, $0.49 \mathrm{~kg} / \mathrm{d} / \mathrm{mi}^{2}$, and $1.01 \mathrm{~kg} / \mathrm{d} / \mathrm{mi}^{2}$, respectively.

In the 36 drainage basins having one or more permitted effluent discharges in the area $3.1 \mathrm{mi}$ upstream from the monitoring station, computed yields of effluent discharge and BOD and TSS were generally less than computed yields for the entire drainage basin. The median yield of effluent discharge computed for the area $3.1 \mathrm{mi}$ upstream from the monitoring station of the 36 drainage basins was less than $0.01 \mathrm{Mgal} / \mathrm{d} / \mathrm{mi}^{2}$, whereas median yields of BOD and TSS in the area $3.1 \mathrm{mi}$ upstream were 0.16 and $0.49 \mathrm{~kg} / \mathrm{d} / \mathrm{mi}^{2}$, respectively. This pattern indicates that most of the effluent discharges in the drainage basins were at a distance greater than $3.1 \mathrm{mi}$ upstream from the monitoring stations.

The PCS data base also contains a Standard Industrial Classification (SIC) code (U.S. Office of Management and Budget, 1987) for each facility having an effluent discharge permit. The SIC code can be used to differentiate between municipal facilities (also known as publicly owned treatment works) and industrial facilities and to determine whether one type of facility was the primary source of effluent in a drainage basin. Municipal facilities are identified by the SIC code 4952; all other SIC codes were considered by the investigators to be nonmunicipal facilities. The percentages of effluent discharge and the BOD and TSS yields from municipal discharges are listed in tables 9 and 10 for the entire drainage basin and for the area $3.1 \mathrm{mi}$ upstream from the monitoring station, respectively.

The SIC information indicates that municipal treatment facilities contributed 61 percent or more of the computed yields of effluent discharge in 28 of the 47 drainage basins having a permitted wastewater discharge (fig. 6). More than one-half of the yields of BOD and TSS were 
Table 11. Yields of effluent, biochemical oxygen demand, and total suspended solids from permitted effluent discharges in New Jersey drainage basins studied, and percentage of yields in each drainage basin from municipal treatment facilities

[BOD, 5-day biochemical oxygen demand; TSS, total suspended solids; Mgal/d/mi², million gallons per day per square mile; $\mathrm{kg} / \mathrm{d} / \mathrm{mi}^{2}$, kilograms per day per square mile; N.A., no information available; -, no wastewater discharges in drainage basin; <, less than. Source: Data from Michael Dillon, New Jersey Department of Environmental Protection, written commun., 1990, and Steve Rubin, U.S. Environmental Protection Agency, written commun., 1991]

\begin{tabular}{|c|c|c|c|c|c|c|c|}
\hline \multicolumn{2}{|l|}{ Drainage basin } & \multicolumn{3}{|c|}{ Yields } & \multicolumn{3}{|c|}{$\begin{array}{l}\text { Percentage of yields in } \\
\text { drainage basin from municipal } \\
\text { treatment facilities }\end{array}$} \\
\hline Monitoring-station name & $\begin{array}{l}\text { Monitoring- } \\
\text { station } \\
\text { number }\end{array}$ & $\begin{array}{l}\text { Wastewater } \\
\mathrm{Mgal} / \mathrm{d} / \mathrm{mi}^{2}\end{array}$ & $\begin{array}{c}\text { BOD } \\
\mathrm{kg} / \mathrm{d} / \mathrm{mi}^{2}\end{array}$ & $\begin{array}{c}\text { TSS } \\
\mathrm{kg} / \mathrm{d} / \mathrm{mi}^{2}\end{array}$ & $\begin{array}{l}\text { Waste- } \\
\text { water }\end{array}$ & BOD & TSS \\
\hline Wallkill River at Franklin & 01367700 & 0.007 & 0.003 & 0.837 & 2.4 & 21.9 & 0 \\
\hline Wallkill River near Sussex & 01367770 & .008 & .115 & .417 & .5 & .2 & 0 \\
\hline Papakating Creek at Sussex & 01367910 & .002 & .186 & .258 & 92.5 & 98.1 & 98.0 \\
\hline Black Creek near Vernon & 01368950 & .007 & .127 & .397 & 0 & 0 & 0 \\
\hline Passaic River near Millington & 01379000 & .005 & .238 & .327 & 99.4 & 99.6 & 99.7 \\
\hline Passaic River near Chatham & 01379500 & .028 & 1.78 & 1.52 & 84.5 & 93.6 & 79.8 \\
\hline Rockaway River at Pine Brook & 01381200 & .047 & .588 & .917 & .4 & 68.7 & 41.6 \\
\hline Whippany River at Morristown & 01381500 & .047 & 2.62 & .645 & 85.3 & 91.3 & 41.7 \\
\hline Whippany River near Pine Brook & 01381800 & .062 & 4.61 & 2.89 & 75.8 & 97.8 & 82.1 \\
\hline Passaic River at Two Bridges & 01382000 & .062 & 4.71 & 3.55 & 62.2 & 53.0 & 50.7 \\
\hline Wanaque River at Wanaque & 01387000 & .001 & .042 & .103 & 90.7 & 98.7 & 99.2 \\
\hline Saddle River at Lodi & 01391500 & .068 & 4.21 & 3.89 & 97.6 & 99.9 & 99.4 \\
\hline SB Raritan River at Middle Valley & 01396280 & .007 & .467 & .597 & 47.7 & 19.7 & 19.7 \\
\hline SB Raritan River at Arch St at High Bridge & 01396535 & .005 & .323 & .413 & 47.7 & 19.7 & 19.7 \\
\hline Mulhockaway Creek at Van Syckel & 01396660 & .001 & .001 & .002 & N.A. & 0 & 0 \\
\hline SB Raritan River at Three Bridges & 01397400 & .009 & .448 & .962 & 64.7 & 51.9 & 28.2 \\
\hline NB Raritan River near Chester & 01398260 & .019 & .944 & .918 & 100 & 100 & 100 \\
\hline NB Raritan River at Burnt Mills & 01399120 & .009 & .318 & 1.39 & 74.0 & 95.7 & 19.1 \\
\hline Rockaway Creek at Whitehouse & 01399700 & .004 & .133 & .389 & 79.0 & 40.7 & 24.1 \\
\hline Lamington (Black) River at Burnt Mills & $0] 399780$ & .010 & .051 & .163 & 13.8 & 41.8 & 22.8 \\
\hline Raritan River at Manville & 01400500 & .010 & .288 & .808 & 34.9 & 45.2 & 17.7 \\
\hline Millstone River at Grovers Mill & 01400650 & .052 & 1.80 & 1.89 & 97.2 & 98.9 & 99.0 \\
\hline Millstone River at Kingston & 01401440 & .020 & 1.80 & 3.08 & 67.7 & 25.4 & 15.6 \\
\hline Beden Brook near Rocky Hill & 01401600 & .006 & .306 & .258 & 21.7 & 7.5 & 7.9 \\
\hline Millstone River at Weston & 01402540 & .029 & 2.13 & 2.95 & 82.8 & 58.5 & 42.0 \\
\hline Manalapan Brook at Federal Rd near Manalapan & 01405340 & .001 & - & .030 & 0 & - & 0 \\
\hline Shark River near Neptune City & 01407705 & .001 & .033 & .033 & 0 & 0 & 0 \\
\hline Jumping Brook near Neptune City & 01407760 & - & - & - & - & - & - \\
\hline Marsh Bog Brook at Squankum & 01407997 & - & - & - & - & - & - \\
\hline Toms River near Toms River & 01408500 & $<.001$ & .011 & .007 & 66.7 & 15.3 & 32.0 \\
\hline Mullica River at outlet of Atsion Lake at Atsion & 01409387 & - & - & - & - & - & - \\
\hline Hammonton Creek at Wescoatville & 01409416 & .040 & 2.89 & 2.84 & 85.3 & 100 & 99.4 \\
\hline Batsto River at Batsto & 01409500 & - & - & - & - & - & - \\
\hline WB Wading River at Maxwell & 01409815 & - & - & - & - & - & - \\
\hline Oswego River at Harrisville & 01410000 & - & - & - & - & - & - \\
\hline EB Bass River near New Gretna & (01410150 & - & - & - & - & - & - \\
\hline Great Egg Harbor River near Sicklerville & 01410784 & .016 & 1.98 & 4.19 & 100 & 100 & 100 \\
\hline Great Egg Harbor River near Blue Anchor & 01410820 & .006 & .801 & 1.69 & 100 & 100 & 100 \\
\hline Great Egg Harbor River at Weymouth & 01411110 & .002 & .194 & .411 & 100 & 99.9 & 100 \\
\hline Maurice River at Norma & 01411500 & .002 & - & .020 & 0 & - & 0 \\
\hline Cohansey River at Seeley & 01412800 & .011 & - & .223 & 0 & - & 0 \\
\hline Paulins Kill at Blairstown & 01443500 & .022 & .211 & .499 & 15.3 & 84.5 & 61.4 \\
\hline Musconetcong River at outlet of Lake Hopatcong & 01455500 & .002 & .027 & .024 & 17.4 & 89.5 & 90.6 \\
\hline Musconetcong River at Beatyestown & 01456200 & .012 & .615 & .667 & 90.9 & 99.8 & 99.0 \\
\hline Musconetcong River at Riegelsville & 01457400 & .015 & 2.05 & 2.00 & 43.9 & 18.4 & 20.0 \\
\hline Wickecheoke Creek at Stockton & 01461300 & .003 & .032 & .060 & 16.1 & 100 & 80.4 \\
\hline Crosswicks Creek at Extonville & 01464500 & .019 & 1.51 & 1.62 & 3.5 & 1.4 & 2.2 \\
\hline Doctors Creek at Allentown & 01464515 & .004 & .327 & .394 & 100 & 100 & 100 \\
\hline SB Rancocas Creek at Vincentown & 01465850 & .002 & .111 & .069 & 66.9 & 49.7 & 42.6 \\
\hline NB Rancocas Creek at Browns Mills & 01465970 & - & - & - & - & - & - \\
\hline McDonalds Branch in Lebanon State Forest & 01466500 & - & - & - & - & - & - \\
\hline NB Rancocas Creek at Pemberton & 01467000 & - & - & - & - & - & - \\
\hline NB Pennsauken Creek near Moorestown & 01467069 & .017 & .688 & .974 & 100 & 100 & 100 \\
\hline SB Pennsauken Creek at Cherry Hill & 01467081 & .038 & 1.72 & .526 & 100 & 100 & 98.4 \\
\hline Cooper River at Norcross Rd at Lindenwold & 01467120 & - & - & - & - & - & - \\
\hline Cooper River at Lawnside & 01467140 & .160 & 8.90 & .803 & 99.6 & 100 & 99.8 \\
\hline SB Big Timber Creek at Blackwood Terrace & 01467329 & $<.001$ & .000 & $<.001$ & 100 & 100 & 100 \\
\hline Raccoon Creek near Swedesboro & 01477120 & .002 & .026 & .057 & 100 & 100 & 100 \\
\hline Oldmans Creek at Porches Mill & 01477510 & - & - & - & - & - & - \\
\hline Salem River at Woodstown & 01482500 & - & - & - & - & - & - \\
\hline
\end{tabular}


Table 12. Yields of effluent, biochemical oxygen demand and total suspended solids from permitted effluent discharges in an area 3.1 miles upstream from the monitoring stations in New Jersey drainage basins studied, and percentage of yields in each drainage basin from municipal treatment facilities

[BOD, 5-day biochemical oxygen demand; TSS, total suspended solids; $\mathrm{Mgal} / \mathrm{d} / \mathrm{mi}^{2}$, million gallons per day per square mile; $\mathrm{kg} / \mathrm{d} / \mathrm{mi}^{2}$, kilograms per day per square mile; N.A., no information available; - , no wastewater discharges in drainage basin; $<$, less than. Source: Data from Michael Dillon, New Jersey Department of Environmental Protection, written commun., 1990; and Steve Rubin, U.S. Environmental Protection Agency, written commun., 1991]

\begin{tabular}{|c|c|c|c|c|c|c|c|}
\hline \multicolumn{2}{|l|}{ Drainage basin } & \multicolumn{3}{|c|}{ Yields } & \multicolumn{3}{|c|}{$\begin{array}{l}\text { Percentage of yields in drainage basin } \\
\text { from municipal treatment facilities }\end{array}$} \\
\hline Monitoring-station name & $\begin{array}{l}\text { Monitoring- } \\
\text { station number }\end{array}$ & $\begin{array}{l}\text { Wastewater } \\
\mathrm{Mgal} / \mathrm{d} / \mathrm{mi}^{2}\end{array}$ & $\frac{\mathrm{BOD}}{\mathrm{kg} / \mathrm{d} / \mathrm{mi}^{2}}$ & $\frac{\mathrm{TSS}}{\mathrm{kg} / \mathrm{d} / \mathrm{mi}^{2}}$ & Wastewater & BOD & TSS \\
\hline Wallkill River at Franklin & 01367700 & 0.008 & - & 1.06 & 0 & - & 0 \\
\hline Wallkill River near Sussex & 01367770 & .009 & 0.002 & .195 & 0 & 0 & 0 \\
\hline Papakating Creek at Sussex & 01367910 & .006 & .481 & .667 & 92.5 & 98.1 & 98.0 \\
\hline Black Creek near Vernon & 01368950 & .003 & .077 & .639 & 0 & 0 & 0 \\
\hline Passaic River near Millington & 01379000 & - & - & - & - & - & - \\
\hline Passaic River near Chatham & 01379500 & .017 & .794 & 1.18 & 91.2 & 97.7 & 70.2 \\
\hline Rockaway River at Pine Brook & 01381200 & $<.001$ & .001 & $<.001$ & 0 & 0 & 0 \\
\hline Whippany River at Morristown & 01381500 & .120 & 6.78 & 1.66 & 85.7 & 91.4 & 41.9 \\
\hline Whippany River near Pine Brook & 01381800 & .033 & 1.52 & 1.50 & 93.3 & 100 & 97.6 \\
\hline Passaic River at Two Bridges & 01382000 & - & - & - & - & - & - \\
\hline Wanaque River at Wanaque & 01387000 & .003 & .110 & .268 & 92.2 & 98.7 & 99.2 \\
\hline Saddle River at Lodi & 01391500 & .003 & - & .004 & 0 & - & 0 \\
\hline SB Raritan River at Middle Valley & 01396280 & .010 & .413 & .628 & 0 & 0 & 0 \\
\hline SB Raritan River at Arch St at High Bridge & 01396535 & - & - & - & - & - & - \\
\hline Mulhockaway Creek at Van Syckel & 01396660 & - & .003 & .005 & - & 0 & 0 \\
\hline SB Raritan River at Three Bridges & 01397400 & .012 & .205 & .298 & 67.1 & 100 & 73.3 \\
\hline NB Raritan River near Chester & 01398260 & .050 & 2.44 & 2.38 & 100 & 100 & 100 \\
\hline NB Raritan River at Burnt Mills & 01399120 & .002 & .026 & .021 & 0 & 0 & 0 \\
\hline Rockaway Creek at Whitehouse & 01399700 & .011 & .344 & 1.01 & 79.0 & 40.7 & 24.1 \\
\hline Lamington (Black) River at Burnt Mills & 01399780 & $<.001$ & .002 & .003 & 89.3 & 53.0 & 78.2 \\
\hline Raritan River at Manville & 01400500 & .007 & .150 & .490 & 0 & 0 & 0 \\
\hline Millstone River at Grovers Mill & 01400650 & .121 & 4.16 & 4.21 & 100 & 100 & 100 \\
\hline Millstone River at Kingston & 01401440 & .004 & .023 & .058 & 0 & 0 & 0 \\
\hline Beden Brook near Rocky Hill & 01401600 & .012 & .731 & .614 & 0 & 0 & 0 \\
\hline Millstone River at Weston & 01402540 & - & - & - & - & - & - \\
\hline Manalapan Brook at Federal Rd near Manalapan & 01405340 & - & - & - & - & - & - \\
\hline Shark River near Neptune City & 01407705 & .001 & .086 & .081 & 0 & 0 & 0 \\
\hline Jumping Brook near Neptune City & 01407760 & - & - & - & - & - & - \\
\hline Marsh Bog Brook at Squankum & 01407997 & - & - & - & - & - & - \\
\hline Toms River near Toms River & 01408500 & - & - & - & - & - & - \\
\hline Mullica River at outlet of Atsion Lake at Atsion & 01409387 & - & - & - & - & - & - \\
\hline Hammonton Creek at Wescoatville & 01409416 & .089 & 7.47 & 7.32 & 100 & 100 & 100 \\
\hline Batsto River at Batsto & 01409500 & - & - & - & - & - & - \\
\hline WB Wading River at Maxwell & 01409815 & - & - & - & - & - & - \\
\hline Oswego River at Harrisville & 01410000 & - & - & - & - & - & - \\
\hline EB Bass River near New Gretna & 01410150 & - & - & - & - & - & - \\
\hline Great Egg Harbor River near Sicklerville & 01410784 & - & - & - & - & - & - \\
\hline Great Egg Harbor River near Blue Anchor & 01410820 & - & - & - & - & - & - \\
\hline Great Egg Harbor River at Weymouth & 01411110 & - & - & - & - & - & - \\
\hline Maurice River at Norma & 01411500 & - & - & - & - & - & - \\
\hline Cohansey River at Seeley & 01412800 & .029 & - & .576 & 0 & - & 0 \\
\hline Paulins Kill at Blairstown & 01443500 & - & - & - & - & - & - \\
\hline Musconetcong River at outlet of Lake Hopatcong & 01455500 & .001 & .064 & .058 & 95.2 & 99.4 & 98.2 \\
\hline Musconetcong River at Beatyestown & 01456200 & .018 & .729 & .639 & 94.1 & 100 & 99.9 \\
\hline Musconetcong River at Riegelsville & 01457400 & .002 & .079 & .050 & 0 & 0 & 0 \\
\hline Wickecheoke Creek at Stockton & 01461300 & .001 & .083 & .124 & 100 & 100 & 100 \\
\hline Crosswicks Creek at Extonville & 01464500 & - & - & - & - & - & - \\
\hline Doctors Creek at Allentown & 01464515 & .011 & .848 & 1.02 & 100 & 100 & 100 \\
\hline SB Rancocas Creek at Vincentown & 01465850 & .004 & .164 & .094 & 93.3 & 87.1 & 80.5 \\
\hline NB Rancocas Creek at Browns Mills & 01465970 & - & - & - & - & - & - \\
\hline McDonalds Branch in Lebanon State Forest & 01466500 & - & - & - & - & - & - \\
\hline NB Rancocas Creek at Pemberton & 01467000 & - & - & - & - & - & - \\
\hline NB Pennsauken Creek near Moorestown & 01467069 & .044 & 1.78 & 2.52 & 100 & 100 & 100 \\
\hline SB Pennsauken Creek at Cherry Hill & 01467081 & N.A. & - & .021 & N.A. & - & 0 \\
\hline Cooper River at Norcross Rd at Lindenwold & 01467120 & - & - & - & - & - & - \\
\hline Cooper River at Lawnside & 01467140 & .216 & 37.5 & 23.8 & 99.2 & 100 & 99.8 \\
\hline SB Big Timber Creek at Blackwood Terrace & 01467329 & $<.001$ & .001 & .001 & 100 & 100 & 100 \\
\hline Raccoon Creek near Swedesboro & 01477120 & .005 & .067 & .149 & 100 & 100 & 100 \\
\hline Oldmans Creek at Porches Mill & 01477510 & - & - & - & - & - & - \\
\hline Salem River at Woodstown & 01482500 & - & - & - & - & - & - \\
\hline
\end{tabular}




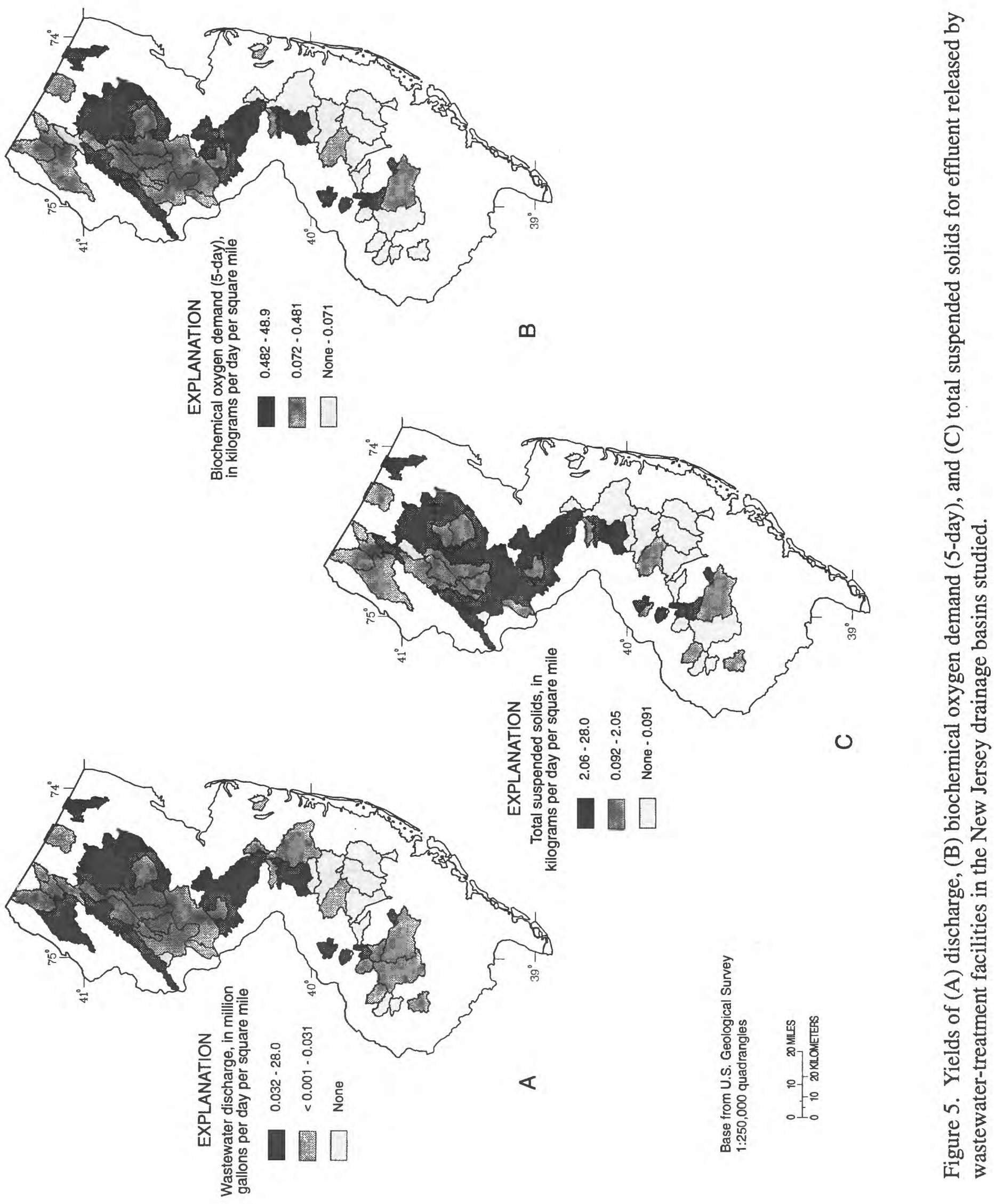




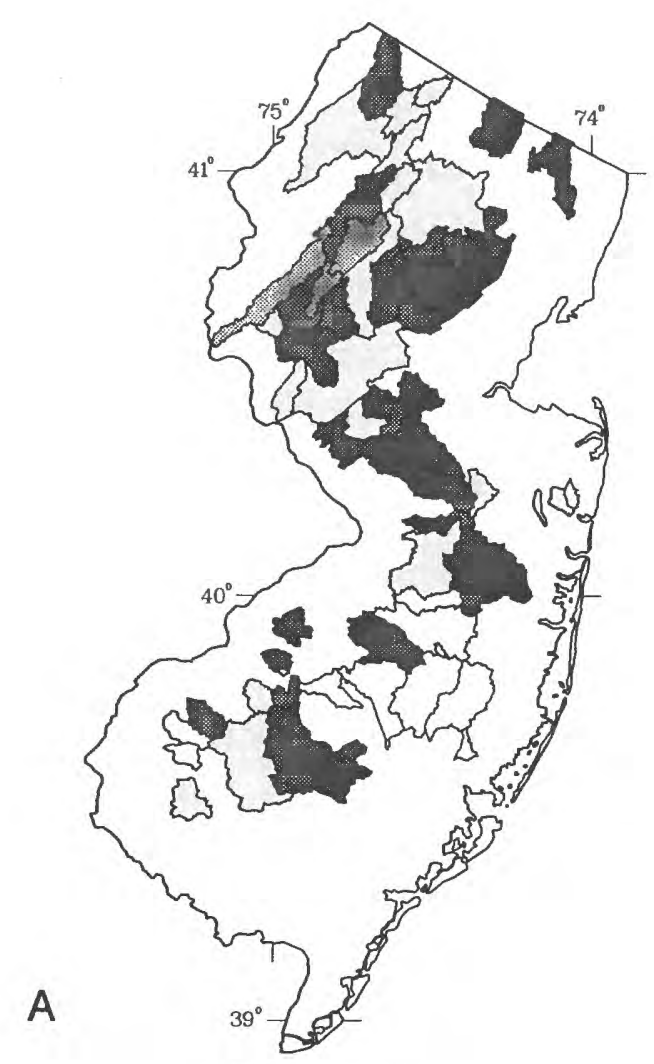

Discharge

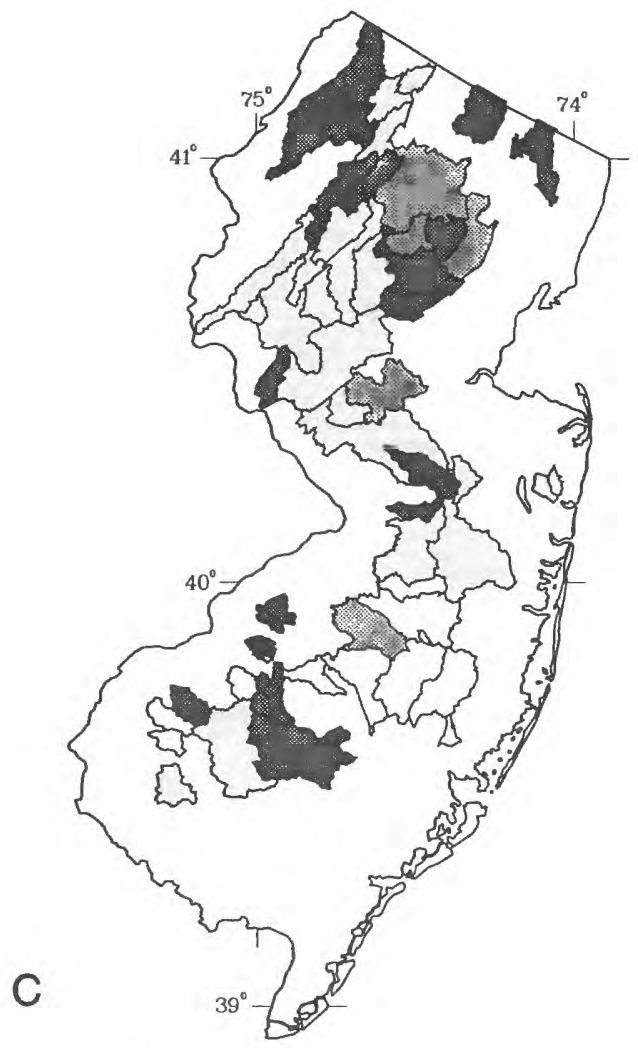

Total suspended solids

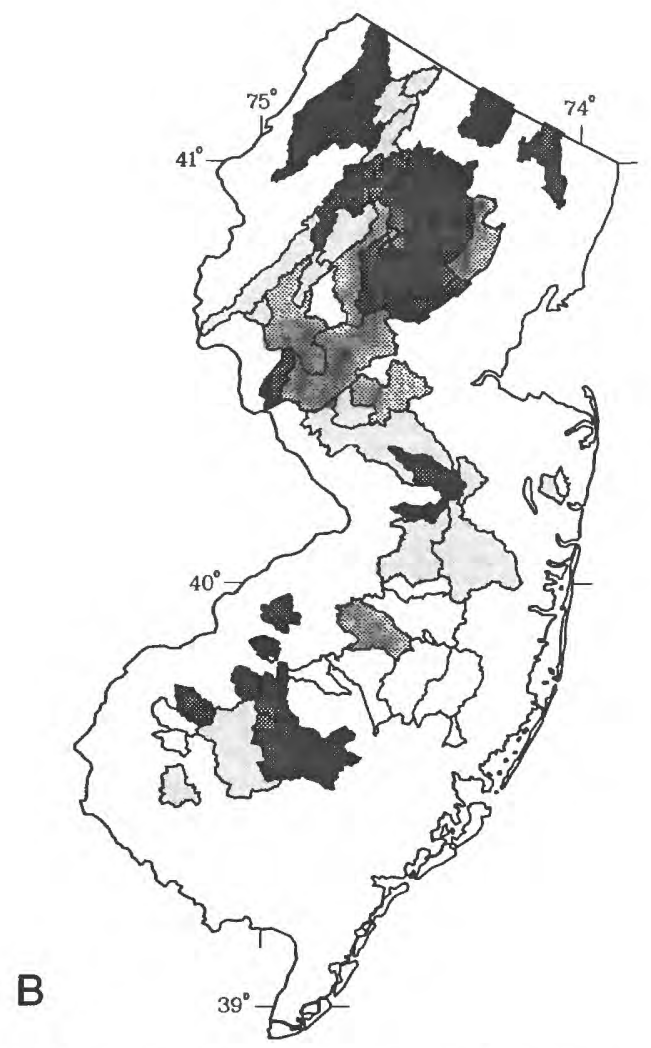

Biochemical oxygen demand (5-day)

\section{EXPLANATION \\ Percentage of total basin yield in effluent from municipal treatment facilities}

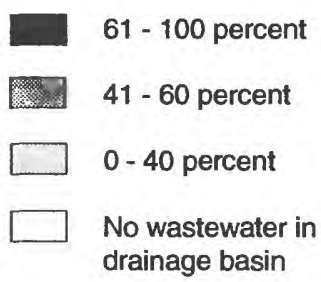

Base from U.S. Geological Survey 1:250,000 quadrangles

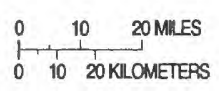

Figure 6. Percentage of yields of (A) discharge, (B) biochemical oxygen demand (5-day), and (C) total suspended solids for effluent released from municipal wastewater-treatment facilities in the New Jersey drainage basins studied. 
from municipal facilities in 26 and 22, respectively, of the 47 drainage basins. Similar ratios of municipal to nonmunicipal sources of effluent were found when determining the sources of effluent in the areas 3.1 mi upstream from the monitoring stations.

\section{Road-Salt Application}

Application of deicing salts to roadways in the winter has the potential for adding large amounts of sodium, calcium, and chloride to surface waters by way of runoff and to ground through infiltration. Road salts have an effect on the concentrations of dissolved constituents in surface waters (Athayde and others, 1983; Harrison and Wilson, 1985; Scott, 1980). Smith and others $(1987 \mathrm{a}, 1987 \mathrm{~b})$ reported that increasing chloride concentrations nationwide were strongly associated with rates of road-salt application. Application of road salts in New Jersey may be linked to the common upward trends in dissolved calcium, sodium, and chloride concentrations in New Jersey (Hay and Campbell, 1990).

Data on the amount of road salt applied to interstate and state highways for 1975-86 were obtained from the New Jersey Department of Transportation (NJDOT) (Alfred Woodrow, New Jersey Department of Transportation, written commun., 1989); (table 13 at back of report). These data account only for NJDOT application of road salts; municipalities, counties, and statehighway authorities do not report their application rates to NJDOT. Road-salt application data from the NJDOT are compiled according to the following four regions of the state:
Region
Counties
1 Hunterdon, Morris, Somerset, Sussex, Warren
2 Bergen, Essex, Hudson, Passaic, Union
3 Mercer, Middlesex, Monmouth, Ocean
4 Atlantic, Burlington, Camden, Cape May, Cumberland, Gloucester, Salem

Road-salt application data from NJDOT were provided by winter season and region as tons of sodium chloride (rocksalt); gallons of calcium chloride in a water solution 32 percent by weight; tons of dry calcium chloride; and tons of a premix of five parts sodium chloride to one part calcium chloride. These data were used to calculate tons of calcium, sodium, and chloride applied per square mile for each drainage basin during each winter season from 1975-76 through 1986-87, based on the percentage of a drainage basin in each NJDOT region. This method requires the assumption of a uniform application rate across each region. The results of these calculations are listed in table 14 (at back of report).

Road-salt application rates for winter 1980-81 were used as a static measure of road-salt applications in the 60 drainage basins. Estimated sodium, chloride, and calcium applications were greatest in the northern part of New Jersey (fig. 7). This part of the State receives more snowfall and has colder temperatures than the southern part of the State. Estimated chloride applications in the 60 drainage basins for winter 1980-81 ranged from 0.83 to $7.64 \mathrm{ton} / \mathrm{mi}^{2}$; estimated sodium applications for winter 1980-81 ranged from 0.47 to 4.1 ton $/ \mathrm{mi}^{2}$; and estimated calcium applications ranged from 0.1 to $1.0 \mathrm{ton} / \mathrm{mi}^{2}$. 


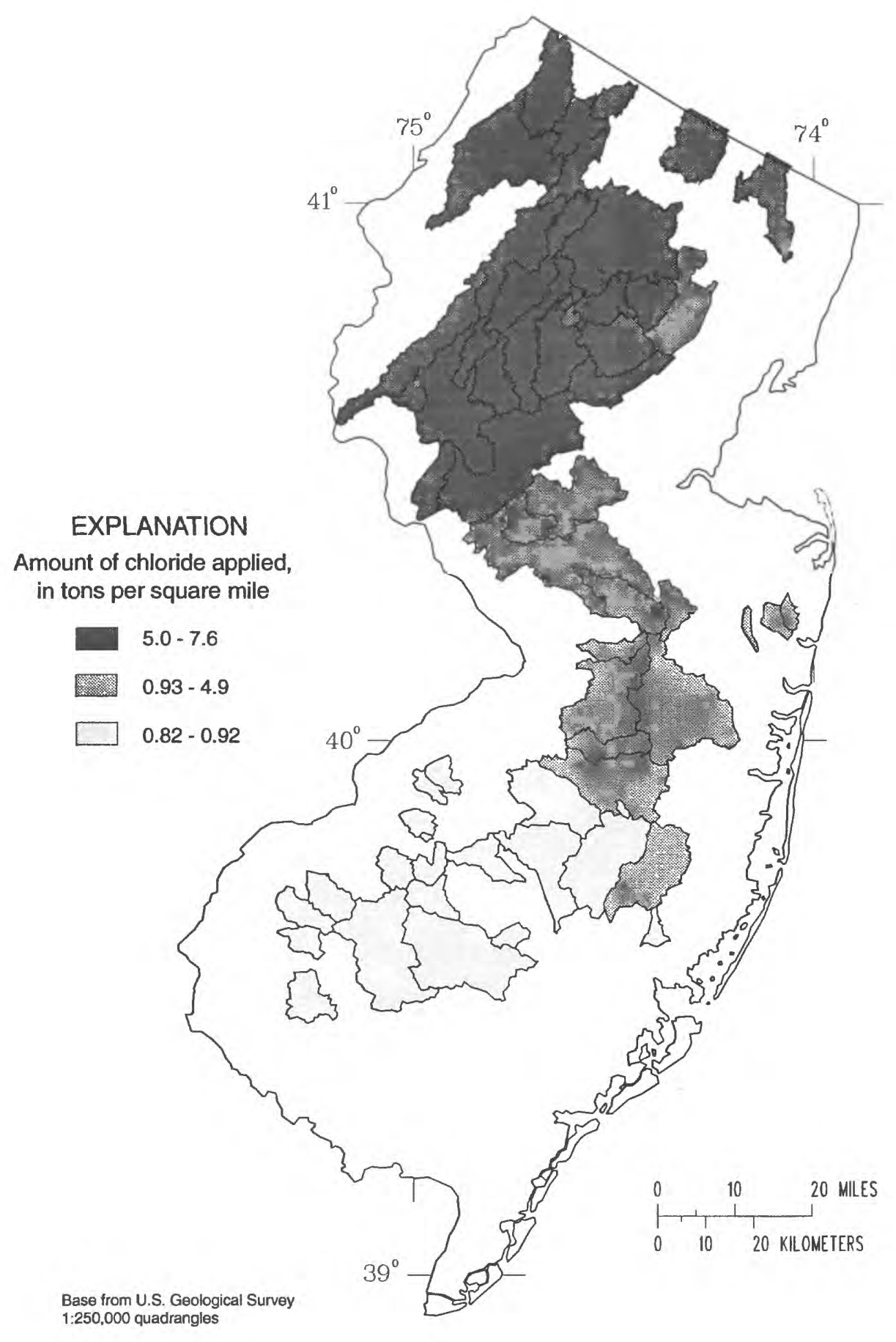

Base from U.S. Geological Survey

Figure 7. Estimated amounts of chloride applied to roadways by the New Jersey Department of Transportation in the New Jersey drainage basins studied, winter 1980-81. 
Trends in the estimated use of road salts in the 60 drainage basins during 1975-87 were calculated to determine whether application rates changed significantly over the period. The trends in the application of calcium, sodium, and chloride for each drainage basin during 197587 were determined by use of the Kendall's tau test for trend (Snedecor and Cochran, 1967). A trend in applications of calcium, sodium and chloride over time was considered to be significant at $\alpha \leq 0.10$.

Results of the trend analyses indicate that calcium application declined significantly in all drainage basins during 1975-87, whereas the estimated application of sodium and chloride increased significantly in 50 of the 60 drainage basins (table 15 and fig. 8). In all drainage basins where an upward trend in sodium application was found, an upward trend in chloride application also was found.

\section{Aaricultural Activities}

Despite being the most densely populated state in the Nation, New Jersey still supports a productive and economically important agricultural industry. Gross farm income in the State grew from $\$ 413.5$ million in 1975 to $\$ 607.5$ million in 1986 (Ferdos Ali, New Jersey Department of Agriculture, written commun., 1992), while farm acreage decreased from approximately 1 million acres to less than 900,000 acres. Cash crops, such as nursery plants, vegetables, fruit, and grain crops, are the most important agricultural products grown in New Jersey. Other agricultural activities that are important locally include horse, dairy, and poultry farming and egg production.

The effects of agricultural activities on water quality in New Jersey are not thought to be as widespread as the effects of other potential sources of water contamination, such as permitted effluent discharges and urban runoff (New Jersey Department of Environmental Protection, 1990). Information describing agricultural activities and their potential environmental effects in the 60 drainage basins were gathered for drainage-basin characterization. This information included agricultural-fertilizer use by county for $1975-85$, cropland soil-erosion rates, and the area of land irrigated.

Agricultural-fertilizer use has been cited as contributing to water-quality problems in many areas of the country (U.S. Environmental Protection Agency, 1984, 1990a). Data on agricultural-fertilizer use by county in New Jersey during 1975-85 (obtained from Richard B. Alexander, U.S. Geological Survey, written commun., 1989) are estimates of tons of nitrogen and phosphorus applied. These estimates were obtained by use of the methodology described by Alexander and Smith (1990).

Drainage-basin estimates of agricultural-fertilizer use were calculated from the county data for each year during 1975-85; these estimates are in table 16 (at back of report). The estimates of fertilizer use by drainage basin were based on the assumption that the nitrogen- and phosphorus-fertilizer applications were uniform across each county. All fertilizer-use estimates of phosphorus and nitrogen were calculated in tons per square mile. 
Table 15. Results of statistical testing for trends in the application of chloride, sodium, and calcium to roadways in the New Jersey drainage basins studied, $1975-87$

[n, sample size for trends testing, equal to the number of yearly values; tau, Kendell's tau computed test statistic; p-value, the probability that the road-salt application values occur randomly rather than occur as result of actual change in application]

\begin{tabular}{|c|c|c|c|c|c|c|c|c|}
\hline \multicolumn{2}{|l|}{ Drainage basin } & \multirow[b]{2}{*}{$\mathbf{n}$} & \multicolumn{2}{|c|}{ Chloride } & \multicolumn{2}{|c|}{ Sodium } & \multicolumn{2}{|c|}{ Calcium } \\
\hline Monitoring-station name & $\begin{array}{l}\text { Monitoring- } \\
\text { station number }\end{array}$ & & $\operatorname{tau}$ & p-value & tau & p-value & tau & p-value \\
\hline Wallkill River at Franklin & 01367700 & 12 & 0.545 & 0.016 & 0.606 & 0.007 & -0.727 & 0.001 \\
\hline Wallkill River near Sussex & 01367770 & 12 & .545 & .016 & .606 & .007 & -.727 & .001 \\
\hline Papakating Creek at Sussex & 01367910 & 12 & .545 & .016 & .606 & .007 & -.727 & .001 \\
\hline Black Creek near Vernon & 01368950 & 12 & .545 & .016 & .606 & .007 & -.727 & .001 \\
\hline Passaic River near Millington & 01379000 & 12 & .545 & .016 & .606 & .007 & -.727 & .001 \\
\hline Passaic River near Chatham & 01379500 & 12 & .545 & .016 & .606 & .007 & -.727 & .001 \\
\hline Rockaway River at Pine Brook & 01381200 & 12 & .545 & .016 & .606 & .007 & -.727 & .001 \\
\hline Whippany River at Morristown & 01381500 & 12 & .545 & .016 & .606 & .007 & -.727 & .001 \\
\hline Whippany River near Pine Brook & 01381800 & 12 & .545 & .016 & .606 & .007 & -.727 & .001 \\
\hline Passaic River at Two Bridges & 01382000 & 12 & .545 & .016 & .606 & .007 & -.727 & .001 \\
\hline Wanaque River at Wanaque & 01387000 & 12 & .576 & .011 & .606 & .007 & -.727 & .001 \\
\hline Saddle River at Lodi & 01391500 & 12 & .576 & .011 & .606 & .007 & -.727 & .001 \\
\hline SB Raritan River at Middle Valley & 01396280 & 12 & .545 & .016 & .606 & .007 & -.727 & .001 \\
\hline SB Raritan River at Arch St at High Bridge & 01396535 & 12 & .545 & .016 & .606 & .007 & -.727 & .001 \\
\hline Mulhockaway Creek at Van Syckel & 01396660 & 12 & .545 & .016 & .606 & .007 & -.727 & .001 \\
\hline SB Raritan River at Three Bridges & 01397400 & 12 & .545 & .016 & .606 & .007 & -.727 & .001 \\
\hline NB Raritan River near Chester & 01398260 & 12 & .545 & .016 & .606 & .007 & -.727 & .001 \\
\hline NB Raritan River at Burnt Mills & 01399120 & 12 & .545 & .016 & .606 & .007 & -.727 & .001 \\
\hline Rockaway Creek at Whitehouse & 01399700 & 12 & .545 & .016 & .606 & .007 & -.727 & .001 \\
\hline Lamington (Black) River at Bumt Mills & 01399780 & 12 & .545 & .016 & .606 & .007 & -.727 & .001 \\
\hline Raritan Rjver at Manville & 01400500 & 12 & .545 & .016 & .606 & .007 & -.727 & .001 \\
\hline Millstone River at Grovers Mill & 01400650 & 12 & .242 & .304 & .273 & .244 & -.515 & .024 \\
\hline Millstone River at Kingston & 01401440 & $1 \overline{2}$ & .242 & .304 & .273 & .244 & -.515 & .024 \\
\hline Beden Brook near Rocky Hill & 01401600 & 12 & .545 & .016 & .606 & .007 & -.727 & .001 \\
\hline Millstone River at Weston & 01402540 & 12 & .242 & .304 & .273 & .244 & -.515 & .024 \\
\hline Manalapan Brook at Federal Rd near Manalapan & 01405340 & 12 & .242 & .304 & .273 & .244 & -.515 & .024 \\
\hline Shark River near Neptune City & 01407705 & 12 & .242 & .304 & .273 & .244 & -.515 & .024 \\
\hline Jumping Brook near Neptune City & 01407760 & 12 & .242 & .304 & .273 & .244 & -.515 & .024 \\
\hline Marsh Bog Brook at Squankum & 01407997 & 12 & .242 & .304 & .273 & .244 & -.515 & .024 \\
\hline Toms River near Toms River & 01408500 & 12 & .242 & .304 & .273 & .244 & -.515 & .024 \\
\hline Mullica River at outlet of Atsion Lake at Atsion & 01409387 & 12 & .545 & .016 & .545 & .016 & -.545 & .016 \\
\hline Hammonton Creek at Wescoatville & 01409416 & 12 & .545 & .016 & .545 & .016 & -.545 & .016 \\
\hline Batsto River at Batsto & 01409500 & 12 & .545 & .016 & .545 & .016 & -.545 & .016 \\
\hline WB Wading River at Maxwell & 01409815 & 12 & .545 & .016 & .545 & .016 & -.545 & .016 \\
\hline Oswego River at Harrisville & 01410000 & 12 & .545 & .016 & .545 & .016 & -.545 & .016 \\
\hline EB Bass River near New Gretna & 01410150 & 12 & .545 & .016 & .545 & .016 & -.545 & .016 \\
\hline Great Egg Harbor River near Sicklerville & 01410784 & 12 & .545 & .016 & .545 & .016 & -.545 & .016 \\
\hline Great Egg Harbor River near Blue Anchor & 01410820 & 12 & .545 & .016 & .545 & .016 & -.545 & .016 \\
\hline Great Egg Harbor River at Weymouth & 01411110 & 12 & .545 & .016 & .545 & .016 & -.545 & .016 \\
\hline Maurice River at Norma & 01411500 & 12 & .545 & .016 & .545 & .016 & -.545 & .016 \\
\hline Cohansey River at Seeley & 01412800 & 12 & .545 & .016 & .545 & .016 & -.545 & .016 \\
\hline Paulins Kill at Blairstown & 01443500 & 12 & .545 & .016 & .606 & .007 & -.727 & .001 \\
\hline Musconetcong River at outlet of Lake Hopatcong & 01455500 & 12 & .545 & .016 & .606 & .007 & -.727 & .001 \\
\hline Musconetcong River at Beatyestown & 01456200 & 12 & .545 & .016 & .606 & .007 & -.727 & .001 \\
\hline Musconetcong River at Riegelsville & 01457400 & 12 & .545 & .016 & .606 & .007 & -.727 & .001 \\
\hline Wickecheoke Creek at Stockton & 01461300 & 12 & .545 & .016 & .606 & .007 & -.727 & .001 \\
\hline Crosswicks Creek at Extonville & 01464500 & 12 & .242 & .304 & .273 & .244 & -.515 & .024 \\
\hline Doctors Creek at Allentown & 01464515 & 12 & .242 & .304 & .273 & .244 & -.515 & .024 \\
\hline SB Rancocas Creek at Vincentown & 01465850 & 12 & .545 & .016 & .545 & .016 & -.545 & .016 \\
\hline NB Rancocas Creek at Browns Mills & 01465970 & 12 & .545 & .016 & .545 & .016 & -.545 & .016 \\
\hline McDonalds Branch in Lebanon State Forest & 01466500 & 12 & .545 & .016 & .545 & .016 & -.545 & .016 \\
\hline NB Rancocas Creek at Pemberton & 01467000 & 12 & .545 & .016 & .545 & .016 & -.545 & .016 \\
\hline NB Pennsauken Creek near Moorestown & 01467069 & 12 & .545 & .016 & .545 & .016 & -.545 & .016 \\
\hline SB Pennsauken Creek at Cherry Hill & 01467081 & 12 & .545 & .016 & .545 & .016 & -.545 & .016 \\
\hline Cooper River at Norcross Rd at Lindenwold & 01467120 & 12 & .545 & .016 & .545 & .016 & -.545 & .016 \\
\hline Cooper River at Lawnside & 01467140 & 12 & .545 & .016 & .545 & .016 & -.545 & .016 \\
\hline SB Big Timber Creek at Blackwood Terrace & 01467329 & 12 & .545 & .016 & .545 & .016 & -.545 & .016 \\
\hline Raccoon Creek near Swedesboro & 01477120 & 12 & .545 & .016 & .545 & .016 & -.545 & .016 \\
\hline Oldmans Creek at Porches Mill & 01477510 & 12 & .545 & .016 & .545 & .016 & -.545 & .016 \\
\hline Salem River at Woodstown & 01482500 & 12 & .545 & .016 & .545 & .016 & -.545 & .016 \\
\hline
\end{tabular}




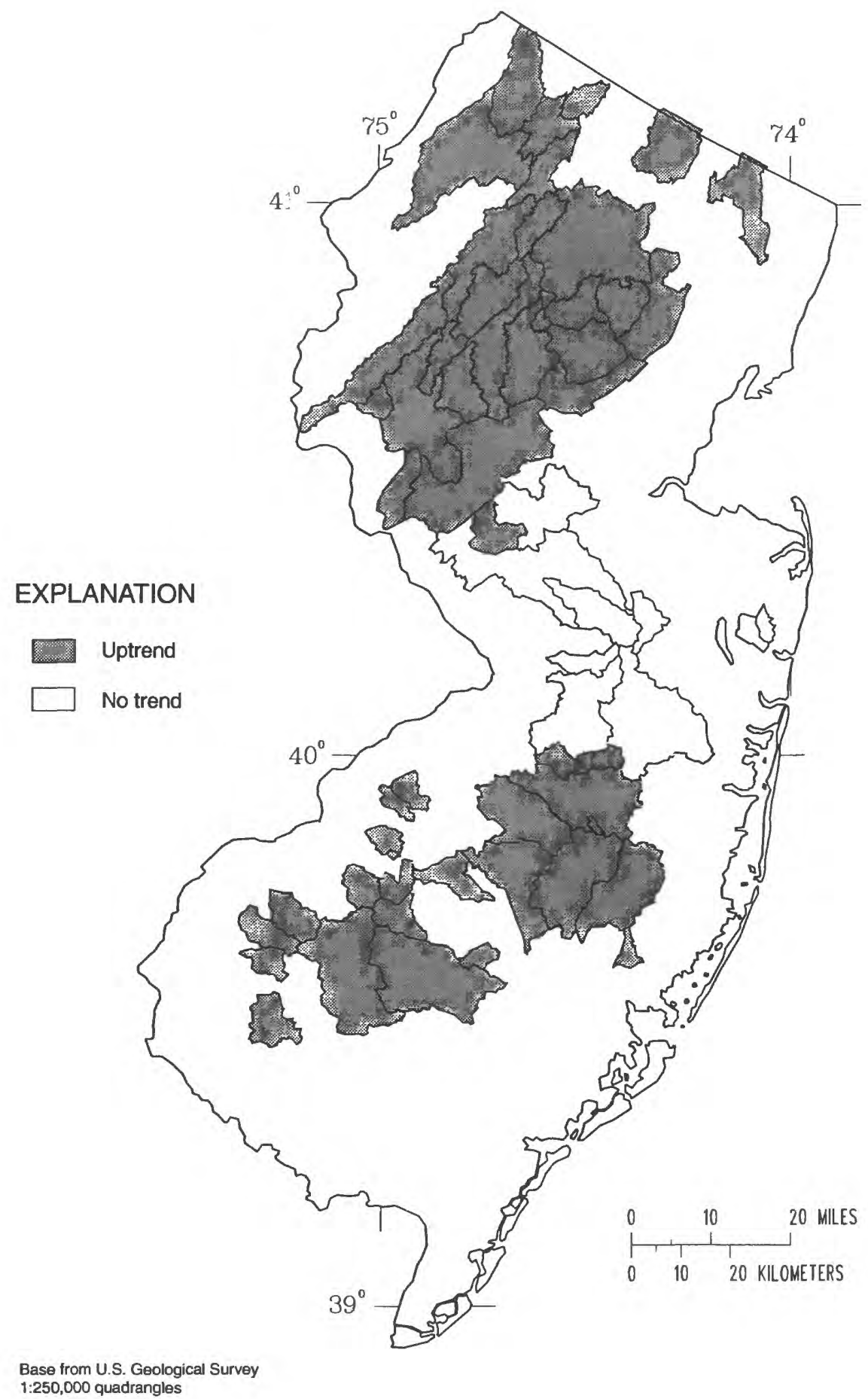

Figure 8. Trends in the estimated amounts of sodium and chloride applied to roadways by the New Jersey Department of Transportation in the New Jersey drainage basins studied, winters 1975-76 through 1986-87. 
In 1980 (chosen as a representative year during WY 1976-86), the greatest amount of phosphorus applied per square mile was in drainage basins in the central and southwestern parts of the State (fig. 9). The drainage basins where phosphorus application rates were the greatest also tended to be the basins where agriculture was the dominant land use (fig. 3). Estimated phosphorus use in 1980 ranged from 0.03 to $7.04 \mathrm{ton} / \mathrm{mi}^{2}$. In the same year, estimated nitrogenfertilizer use in the 60 drainage basins ranged from 0.03 to 9.48 ton $/ \mathrm{mi}^{2}$. Drainage basins where areal applications of phosphorus fertilizer were greatest tended to be basins where areal applications of nitrogen fertilizer also were greatest.

Drainage-basin estimates of nitrogen- and phosphorus-fertilizer applications for 1975-85 were tested for time-series trends to determine whether the amount applied increased or decreased significantly during the period. Trends were identified with the Kendall's tau test for trend; a significant trend was identified at $\alpha<0.1$. Results of the trend testing (table 17) show that estimated nitrogen fertilizer applications significantly increased in all 60 drainage basins, whereas estimated phosphorus applications declined in 52 of the 60 drainage basins. The eight drainage basins where no trend in phosphorus- fertilizer application was found also had the smallest application rates and were generally urbanized. Possible causes for the increases in nitrogen-fertilizer use and decreases in phosphorus-fertilizer use during the period include the following: the drop in cost of nitrogen fertilizer coupled with the rise in cost of phosphorus fertilizer over time; the fact that nitrogen fertilizers are generally favored by farmers because crop response is stronger than for phosphorus fertilizers; and the amount of phosphorus naturally in New Jersey soils tends to be adequate for most crops (David Smart, Natural Resources Conservation Service, oral commun., 1993).

Estimates of cropland soil-erosion rates and of the amount of land under agricultural irrigation in predominantly agricultural or forested areas of New Jersey were made in the early 1980's as part of the U.S. Department of Agriculture's (USDA) Soil Erosion, Sediment, and Animal Waste (SEASAW) Study (U.S. Department of Agriculture, 1986). The soil-erosion and irrigated-acreage estimates in the SEASAW study are based on data collected by the USDA at random locations in 20 large drainage basins of the State for the purpose of providing statistically valid estimates at the drainage-basin level (U.S.Department of Agriculture, 1986). Of the 60 drainage basins tested for water-quality trends, 39 are among the drainage basins in the SEASAW study. The soil-erosion and irrigated-acreage estimates from the SEASAW study for these 39 drainage basins are listed in table 18 .

Of the basins investigated, the South Branch of the Raritan River Basin had the greatest cropland soil-erosion rate at 10 ton/acre/yr, followed by the Musconetcong River Basin at 9.8 ton/acre/yr (fig. 10). In most drainage basins, cropland soil-erosion rates were greater than 5 ton/ acre/yr; soil-erosion rates greater than 5 ton/acre/yr generally result in a net loss of soil (Thomas Drewes, U.S. Soil Conservation Service, oral commun., 1989).

Irrigated acreage generally was a small percentage of the area of the drainage basins analyzed. Basins draining to the Delaware River in southwestern New Jersey had the greatest percentage of irrigated land: approximately 14 percent of each of the basins (table 13). The percentage of the drainage basin irrigated in all remaining basins was less than 10 percent. 


\section{EXPLANATION}

Amount of phosphorus fertilizer applied, in tons per square mile

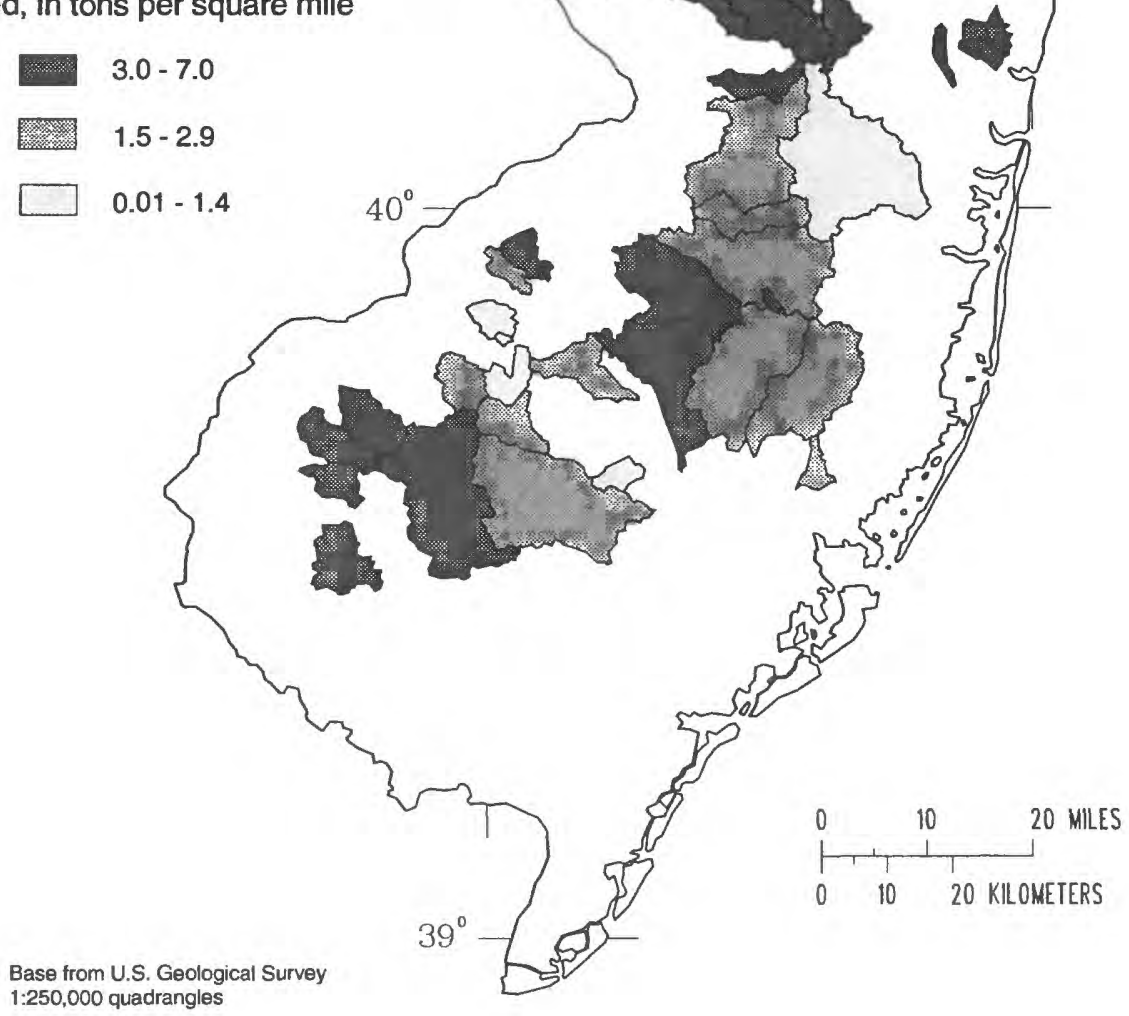

Figure 9. Estimated amounts of phosphorus fertilizer applied in the New Jersey drainage basins studied, 1980. 
Table 17. Results of statistical testing for trends in the use of phosphorus and nitrogen fertilizers in the New Jersey drainage basins studied, 1975-85

[n, sample size for trends testing, equal to the number of yearly values; tau, Kendell's tau computed test statistic; p-value, the probability that fertilizer application values occur randomly rather than occur as a result of actual changes in application]

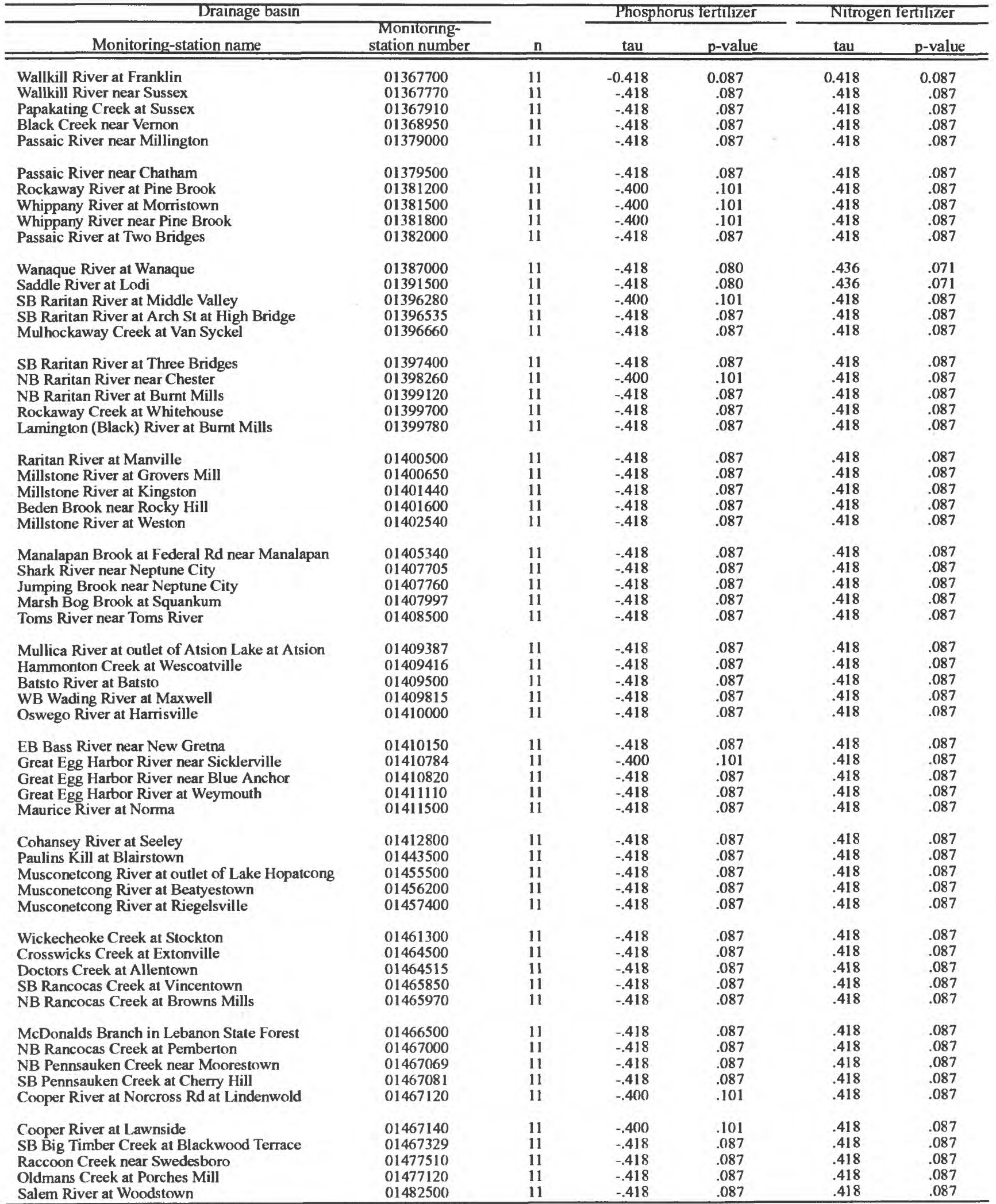


Table 18. Cropland soil-erosion rates and estimated acreage irrigated in 39 of the New Jersey drainage basins studied, 1986

[Data from U.S. Department of Agriculture (1986); ton/acre/yr, tons per acre per year; <, less than]

\begin{tabular}{|c|c|c|c|c|}
\hline \multicolumn{2}{|l|}{ Drainage basin } & \multirow{2}{*}{$\begin{array}{l}\text { Rate of } \\
\text { cropland } \\
\text { erosion } \\
\text { (ton/acre/yr) }\end{array}$} & \multicolumn{2}{|c|}{ Estimated irrigated acreage } \\
\hline Monitoring-station name & $\begin{array}{l}\text { Monitoring- } \\
\text { station number }\end{array}$ & & $\begin{array}{l}\text { Number of } \\
\text { acres }\end{array}$ & $\begin{array}{l}\text { Percentage of } \\
\text { drainage- } \\
\text { basin area }\end{array}$ \\
\hline Wallkill River at Franklin & 01367700 & 3.6 & 0 & 0 \\
\hline Wallkill River near Sussex & 01367770 & 3.6 & 0 & 0 \\
\hline Papakating Creek at Sussex & 01367910 & 3.6 & 0 & 0 \\
\hline Black Creek near Vernon & 01368950 & 3.6 & 0 & 0 \\
\hline SB Raritan River at Middle Valley & 01396280 & 10.0 & 172 & $<1$ \\
\hline SB Raritan River at Arch St at High Bridge & 01396535 & 10.0 & 251 & $<1$ \\
\hline Mulhockaway Creek at Van Syckel & 01396660 & 10.0 & 43 & $<1$ \\
\hline SB Raritan River at Three Bridges & 01397400 & 10.0 & 661 & $<1$ \\
\hline NB Raritan River near Chester & 01398260 & 8.1 & 0 & 0 \\
\hline NB Raritan River at Burnt Mills & 01399120 & 8.1 & 0 & 0 \\
\hline Rockaway Creek at Whitehouse & 01399700 & 8.1 & 0 & 0 \\
\hline Lamington (Black) River at Burnt Mills & 01399780 & 8.1 & 0 & 0 \\
\hline Millstone River at Grovers Mill & 01400650 & 7.2 & 1,762 & 6 \\
\hline Millstone River at Kingston & 01401440 & 7.2 & 6,983 & 6 \\
\hline Beden Brook near Rocky Hill & 01401600 & 7.2 & 1,121 & 6 \\
\hline Millstone River at Weston & 01402540 & 7.2 & 10,927 & 6 \\
\hline Manalapan Brook at Federal Rd near Manalapan & 01405340 & 7.2 & 174 & $<1$ \\
\hline Marsh Bog Brook at Squankum & 01407997 & 4.8 & 0 & 0 \\
\hline Maurice River at Norma & 01411500 & 7.2 & 5,735 & 8 \\
\hline Cohansey River at Seeley & 01412800 & 7.1 & 2,477 & 8 \\
\hline Paulins Kill at Blairstown & 01443500 & 3.6 & 0 & 0 \\
\hline Musconetcong River at outlet of Lake Hopatcong & 01455500 & 9.8 & 51 & $<1$ \\
\hline Musconetcong River at Beatyestown & 01456200 & 9.8 & 184 & $<1$ \\
\hline Musconetcong River at Riegelsville & 01457400 & 9.8 & 240 & $<1$ \\
\hline Wickecheoke Creek at Stockton & 01461300 & 9.8 & 0 & 0 \\
\hline Crosswicks Creek at Extonville & 01464500 & 5.8 & 2,379 & 5 \\
\hline Doctors Creek at Allentown & 01464515 & 5.8 & 508 & 5 \\
\hline SB Rancocas Creek at Vincentown & 01465850 & 4.0 & 1,029 & 2 \\
\hline NB Rancocas Creek at Browns Mills & 01465970 & 4.0 & 437 & 2 \\
\hline McDonalds Branch in Lebanon State Forest & 01466500 & 4.0 & 38 & 2 \\
\hline NB Rancocas Creek at Pemberton & 01467000 & 4.0 & 1,882 & 2 \\
\hline NB Pennsauken Creek near Moorestown & 01467069 & 5.1 & 1,173 & 14 \\
\hline SB Pennsauken Creek at Cherry Hill & 01467081 & 5.1 & 823 & 14 \\
\hline Cooper River at Norcross Rd at Lindenwold & 01467120 & 5.1 & 102 & 14 \\
\hline Cooper River at Lawnside & 01467140 & 5.1 & 1,162 & 14 \\
\hline SB Big Timber Creek at Blackwood Terrace & 01467329 & 5.1 & 1,749 & 14 \\
\hline Raccoon Creek near Swedesboro & 01477120 & 5.1 & 2,463 & 14 \\
\hline Oldmans Creek at Porches Mill & 01477510 & 5.1 & 809 & 6 \\
\hline Salem River at Woodstown & 01482500 & 8.5 & 562 & 6 \\
\hline
\end{tabular}




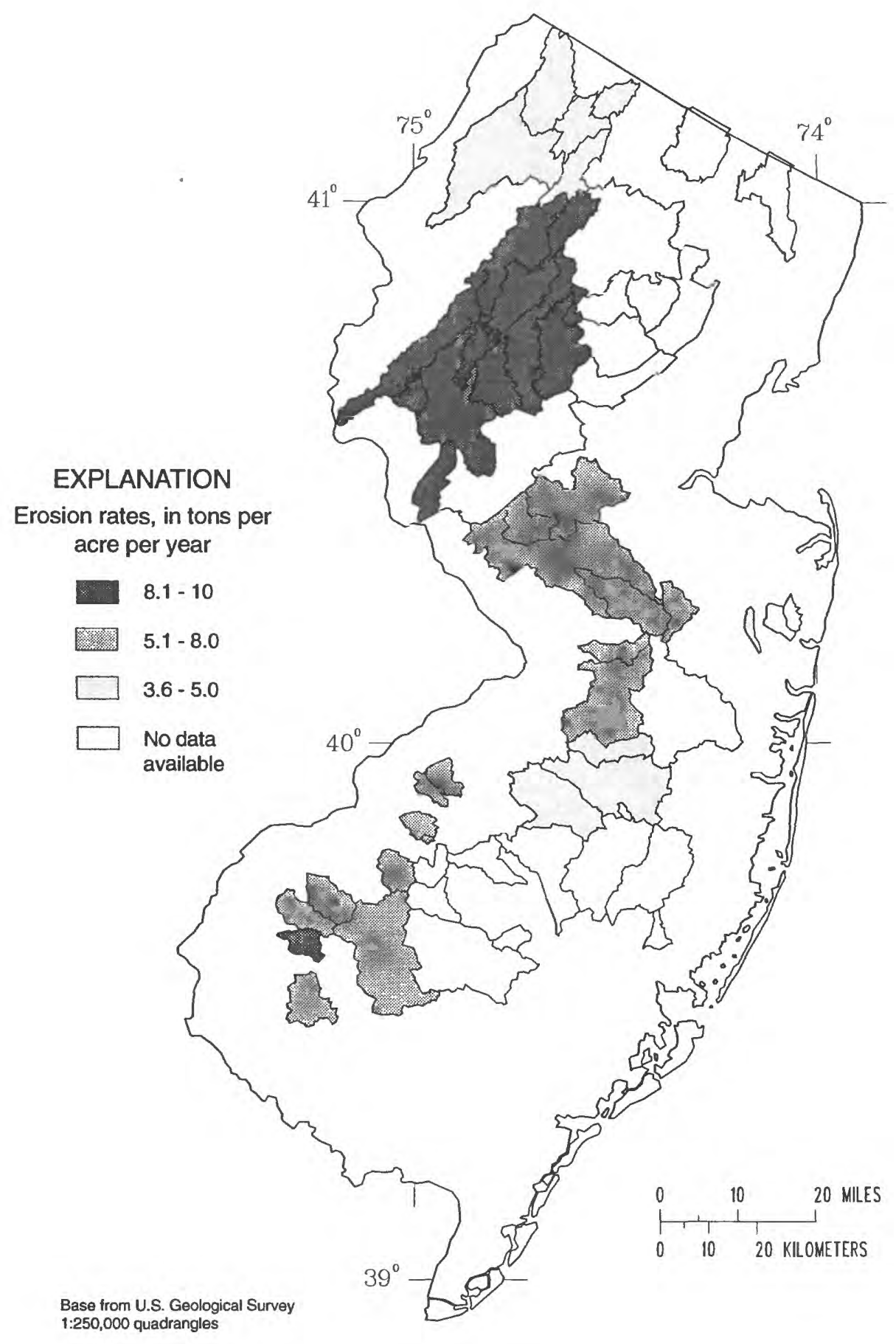

Figure 10. Estimated cropland soil-erosion rates in 39 of the New Jersey drainage basins studied, early 1980's. 


\section{METHODS OF STUDY}

\section{Statistical Methods for Associating Water-Quality Trends and Drainage-Basin characteristics}

Associations between water-quality trends and drainage-basin characteristics were determined by means of contingency-table analyses. A contingency-table analysis (Snedecor and Cochran, 1967) is designed to test the association of one variable (for example, trends for a streamwater constituent or property) with a second variable (for example, a basin characteristic). A contingency-table analysis is not intended to imply a statistical cause-and- effect relation, as might be implied by results from regression or correlation analysis. Smith and others (1987a, 1987b) and Lettenmaier and others (1991) used contingency-table analyses in their interpretations of nationwide water-quality trends. In this study also, contingency-table analyses were used to identify associations among the trends of the 15 constituents and properties with each other and to determine whether each trend had a seasonal component.

Contingency tables associating water-quality trends to basin characteristics were usually constructed in $3 \times 3$ arrays: the trend results for a constituent or property (upward trend, downward trend, or no trend) against three categorical definitions of a drainage-basin characteristic. The null hypothesis, $\mathrm{H}_{0}$, of the contingency-table analysis is that the row classification (water-quality trend) is independent of the column classification (basin characteristic).

Contingency tables are used to compare the expected count for each cell in the array with the observed count. The expected count for a cell is the count one would expect to find, given the assumption of independence, if only the row and column counts are known. If the differences between expected and observed counts are small, then the null hypothesis is accepted. If the difference between the observed and expected counts is sufficiently large (larger than the test statistic at a given $\alpha$ level), then the null hypothesis is rejected. In this case, a significant statistical association between the row and column classifications is considered to exist. Significance of association between two variables in a contingency table is tested by use of a chi-square statistic or by the exact significance through enumeration of all possible table configurations.

Associations were considered to be statistically significant at $\alpha \leq 0.10$; this is the probability that an apparent association between the two variables could have resulted solely by chance arrangement of the data rather than through probable association between the two variables.

If more than one cell in a contingency table had an expected frequency of less than 1 , or if more than 20 percent of the cells had an expected frequency of less than 5 , then the observed data were not considered to be distributed sufficiently to represent a chi-square distribution (Cochran, 1952). Either arrangement can result in inaccurate probabilities from chi-square testing. When this insufficient distribution of expected values in the contingency table occurred, the exact significance was determined through enumeration of all possible table configurations (Cochran, 1952). 
All drainage-basin characteristics analyzed for association with the water-quality trends were classified into three categories for use in contingency tables. If the basin characteristic was described in terms of a continuous value, such as the amount of road salt applied in a basin, then the category assigned to a basin was based on the 33rd and 67th percentiles of the ranked values for all basins. If the basin characteristic was a categorical term (such as physiographic province), then the terms were grouped into three classes on the basis of similar characteristics. A description of the categories of drainage-basin characteristics used in contingency-table analyses is given in table 19 .

One set of contingency-table variables, seasonal trends for selected constituents and properties, was divided into six categories. Each category was a water-quality trend result for data from a 2-month period (or season) during WY 1976-86. This category resulted in a 36 array; WY 1976-86 trend result reported by Hay and Campbell (1990) against the trend result for each 2-month period. All contingency-table results are listed in table 20 (at back of report).

As mentioned previously, a contingency-table analysis test for the presence or absence of statistical association between water-quality trends and a drainage-basin characteristic does not determine cause-and-effect relations between the variables. In addition, streamwater quality can be affected by many factors, some of which could not be quantified and tested for association. As a result, the associations identified in this report by the contingency-table analyses are designed to indicate which drainage-basin characteristics and activities may have affected the trends in water quality in New Jersey streams. These associations can serve as the basis for future cause-and-effect investigations of water quality.

\section{Methods for Selecting Draingae Basins}

In using chi-square contingency-table analysis, one assumes that the variable data are independent and random. In 10 of the 60 drainage basins analyzed, 2 or more monitoring stations tested for trends in water-quality data are on the same stream. This can mean that the water-quality data, and therefore, trends in those data, may not be independent. Water-quality trends that are related to other water-quality trends on the same stream had to be eliminated before contingency-table analysis could be done. Correlation analyses of data for each constituent and property measured at the monitoring stations on the same stream were done to identify which water-quality trends were not independent.

The water-quality data used in the correlation analysis were the same data used in the trend analysis by Hay and Campbell (1990). Where significant correlation was found in the data for a particular constituent at two monitoring stations, one monitoring station was dropped from the data base. As a result, a unique list of monitoring stations (or drainage basins) was generated for each constituent and property for use in the contingency-table analysis.

A nonparametric statistical test for data correlation, Spearman's Rank- Correlation Coefficient (Conover, 1980), was used to identify the presence of correlation in the waterquality data. This statistical method identifies relation between variables on the basis of ranks of the data for each variable; no assumptions on the distribution of the data are required. To ensure 
Table 19. Categorical definitions of drainage-basin characteristics as used in contingency-table analysis of data for the New Jersey drainage basins studied

[The category definitions for continuous variables were calculated by use of the 33rd and 67th percentiles of the distribution of the data; $\mathrm{mi}^{2}$, square miles; $\mathrm{Mgal} / \mathrm{d} / \mathrm{mi}^{2}$, million gallons per day per square mile; $\mathrm{kg} / \mathrm{d} / \mathrm{mi}^{2}$, kilograms per day per square mile; ton $/ \mathrm{mi}^{2}$, tons per square mile]

\begin{tabular}{|c|c|c|c|}
\hline \multirow{2}{*}{ Basin characteristic } & \multicolumn{3}{|c|}{ Category } \\
\hline & 1 & 2 & 3 \\
\hline Drainage-basin size $\left(\mathrm{mi}^{2}\right)$ & $1.13-25.3$ & $26.0-68.8$ & $69.0-490$ \\
\hline Physiographic province & Coastal Plain & Piedmont & $\begin{array}{l}\text { New England; } \\
\text { Valley and Ridge }\end{array}$ \\
\hline Dominant land-use type & Urban & Forest & Agriculture \\
\hline \multicolumn{4}{|l|}{ Population } \\
\hline a. 1986 population (persons) & $48-9,482$ & $9,483-29,780$ & $29,781-424,547$ \\
\hline b. 1986 population density (persons per square mile) & $21-303$ & $304-663$ & $664-2,889$ \\
\hline $\begin{array}{l}\text { c. Change in population density, 1975-86 (persons per } \\
\text { square mile) }\end{array}$ & $-75-+29$ & $+30-+70$ & $+71-+438$ \\
\hline \multicolumn{4}{|l|}{ Permitted wastewater discharge } \\
\hline a. Change in number of treatment facilities, $1975-86$ & Decrease & No change & Increase \\
\hline \multicolumn{4}{|l|}{ b. Wastewater yields in drainage basin } \\
\hline 1. Wastewater discharge (Mgal/d/mi $)$ & 0.000 & $<0.001-0.031$ & $0.032-28.0$ \\
\hline 2. Biochemical oxygen demand, 5 -day $\left(\mathrm{kg} / \mathrm{d} / \mathrm{mi}^{2}\right)$ & $0.000-0.071$ & $0.072-0.481$ & $0.482-48.9$ \\
\hline 3. Total suspended solids $\left(\mathrm{kg} / \mathrm{d} / \mathrm{mi}^{2}\right)$ & $0.000-0.091$ & $0.092-2.05$ & $2.06-28.0$ \\
\hline $\begin{array}{l}\text { c. Percentage of wastewater flow, biological oxygen } \\
\text { demand, and total suspended soilds originating from } \\
\text { municipal facilities }\end{array}$ & $0-40$ & $41-60$ & $61-100$ \\
\hline \multicolumn{4}{|l|}{$\begin{array}{l}\text { d. Wastewater yields in area } 3.1 \text { miles upstream from the } \\
\text { monitoring station }\end{array}$} \\
\hline 1. Wastewater discharge $\left(\mathrm{Mgal} / \mathrm{d} / \mathrm{mi}^{2}\right)$ & $0.000-0.005$ & $0.006-0.012$ & $0.013-0.216$ \\
\hline 2. Biochemical oxygen demand, 5-day $\left(\mathrm{kg} / \mathrm{d} / \mathrm{mi}^{2}\right)$ & $0.000-0.067$ & $0.068-0.671$ & $0.672-37.5$ \\
\hline 3. Total suspended solids $\left(\mathrm{kg} / \mathrm{d} / \mathrm{mi}^{2}\right)$ & $0.000-0.093$ & $0.094-0.710$ & $0.711-23.8$ \\
\hline \multicolumn{4}{|l|}{ Road-salt application in winter, $1980-81$} \\
\hline a. Calcium (ton $\left./ \mathrm{mi}^{2}\right)$ & $0.09-0.10$ & $0.11-0.63$ & $0.64-1.1$ \\
\hline b. Sodium (ton $/ \mathrm{mi}^{2}$ ) & $0.46-0.52$ & $0.53-2.7$ & $2.8-4.1$ \\
\hline c. Chloride (ton $\left./ \mathrm{mi}^{2}\right)$ & $0.82-0.92$ & $0.93-4.9$ & $5.0-7.6$ \\
\hline \multicolumn{4}{|l|}{ Agricultural activities } \\
\hline \multicolumn{4}{|l|}{ a. 1980 fertilizer application $\left(\operatorname{ton} / \mathrm{mi}^{2}\right)$} \\
\hline 1. Phosphorus & $0.01-1.4$ & $1.5-2.9$ & $3.0-7.0$ \\
\hline 2. Nitrogen & $0.02-1.9$ & 2.0-3.9 & $4.0-9.5$ \\
\hline b. Cropland soil erosion rate (tons per acre per year) & $3.6-5.0$ & $5.1-8.0$ & $8.1-10$ \\
\hline c. Irrigated land in basin (percent of drainage basin) & $0.00-1.00$ & $1.01-5.00$ & $5.01-14.0$ \\
\hline
\end{tabular}


that the water-quality data were collected under similar hydrologic conditions, the data from the two monitoring stations used in the correlation analysis had to be collected on the same day. Significant correlation was identified at $\alpha=0.05$.

Before the correlation analysis was done, all remark codes in the water-quality data were eliminated. Data with "less than" remark codes were assigned half the detection limit, and data with "greater than" remark codes were assigned the highest detection value plus 1 .

When the data for a constituent or property were significantly correlated at two or more monitoring stations, the following criteria were applied to select the monitoring station for use in the contingency-table analysis:

- If either no trend or a trend in the same direction was found for a constituent or property at two monitoring stations on the same stream, then the downstream station was selected.

- If an upward trend or a downward trend was found for a constituent or property at one station but no trend was found at the second station, then the station where the trend was found was selected regardless of its location on the stream.

- If a constituent or property was tested for trends at only one of two stations, then the station analyzed for trends was selected.

- If three stations were on the same stream, then each pair was tested as above. If data at the most upstream and most downstream stations were both correlated with the data at the middle station but not with each other, and if trends were found at all three stations, then the middle station was excluded.

Results of the correlation analysis are listed in table 21 (at back of report). Correlation analyses resulted in the elimination of one to eight monitoring stations depending on the constituent or property (table 22). Specific conductance, $\mathrm{pH}$, and concentrations of dissolved oxygen and total organic carbon, were most frequently correlated on the same stream. The number of drainage basins remaining in the data set after the correlation analyses ranged from 17 for concentrations of total ammonia to 52 for concentrations of dissolved sodium (table 22). The water-quality trends for these drainage basins (Hay and Campbell, 1990) are listed in table 23 (at back of report) and shown in figures 11-13.

\section{ASSOCIATIONS BETWEEN WATER-QUALITY TRENDS AND DRAINAGE-BASIN CHARACTERISTICS}

Contingency-table analyses reveal that trends of many of the streamwater constituents and properties found in New Jersey streams during WY 1976-86 appear to be affected by the drainage-basin characteristics described in this report. Test results show that all of the drainagebasin characteristics were statistically associated with the trends of one or more of the 13 constituents or 2 physical properties (table 24). A detailed discussion of the results of 
Table 22. Summary of correlation-test results on water-quality data collected at New Jersey stream-monitoring stations whose trends were used in contingency-table analysis

[ $\mu \mathrm{S} / \mathrm{cm}$, microsiemens per centimeter at 25 degrees Celsius; $\mathrm{mg} / \mathrm{L}$, milligrams per liter; MPN/100 mL, most probable number per 100 milliliter]

\begin{tabular}{|c|c|c|c|}
\hline Constituent or property (units) & $\begin{array}{c}\text { Number of } \\
\text { monitoring } \\
\text { stations tested for } \\
\text { correlation }\end{array}$ & $\begin{array}{l}\text { Number of } \\
\text { monitoring } \\
\text { stations } \\
\text { eliminated } \\
\text { because of data } \\
\text { correlation }\end{array}$ & $\begin{array}{c}\text { Number of } \\
\text { monitoring } \\
\text { stations whose } \\
\text { trends were tested } \\
\text { for association } \\
\text { with basin } \\
\text { characteristics }\end{array}$ \\
\hline Specifc conductance $(\mu \mathrm{S} / \mathrm{cm})$ & 23 & 7 & 50 \\
\hline Dissolved oxygen $(\mathrm{mg} / \mathrm{L})$ & 23 & 8 & 47 \\
\hline Biochemical oxygen demand, 5-day (mg/L) & 23 & 5 & 39 \\
\hline pH (standard units) & 23 & 6 & 48 \\
\hline Total nitrogen, as $\mathrm{N}(\mathrm{mg} / \mathrm{L})$ & 23 & 1 & 20 \\
\hline Total ammonia, as N (mg/L) & 23 & 2 & 17 \\
\hline Total phosphorus, as $\mathrm{P}(\mathrm{mg} / \mathrm{L})$ & 23 & 3 & 39 \\
\hline Total organic carbon $(\mathrm{mg} / \mathrm{L})$ & 23 & 7 & 43 \\
\hline Dissolved calcium (mg/L) & 23 & 4 & 50 \\
\hline Dissolved magnesium $(\mathrm{mg} / \mathrm{L})$ & 23 & 4 & 50 \\
\hline Dissolved sodium (mg/L) & 23 & 4 & 52 \\
\hline Dissolved potassium (mg/L) & 23 & 4 & 51 \\
\hline Dissolved chloride $(\mathrm{mg} / \mathrm{L})$ & 23 & 5 & 51 \\
\hline Fecal coliform (MPN/100 mL) & 23 & 3 & 48 \\
\hline Fecal streptococcus (MPN/100 mL) & 23 & 2 & 45 \\
\hline
\end{tabular}



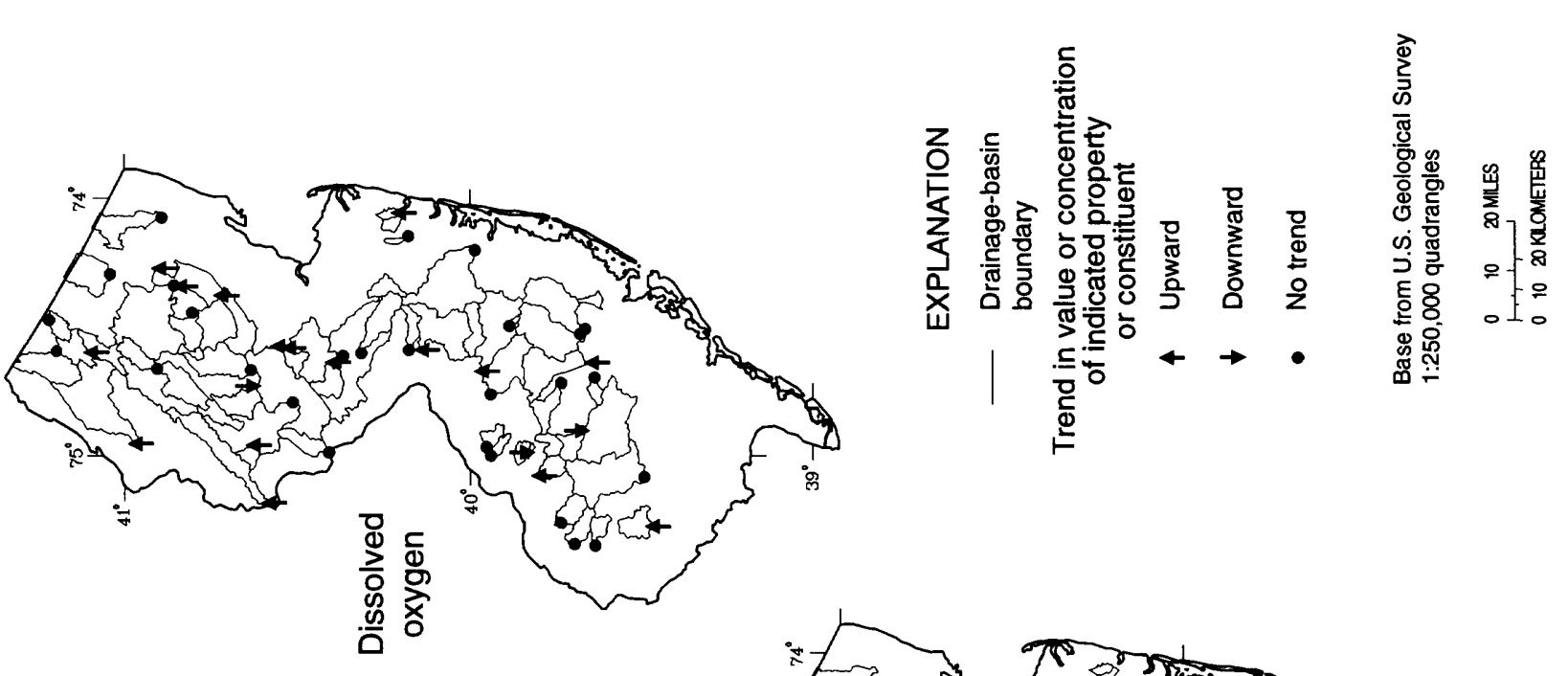

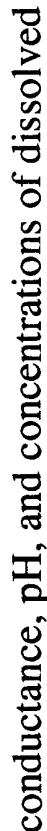

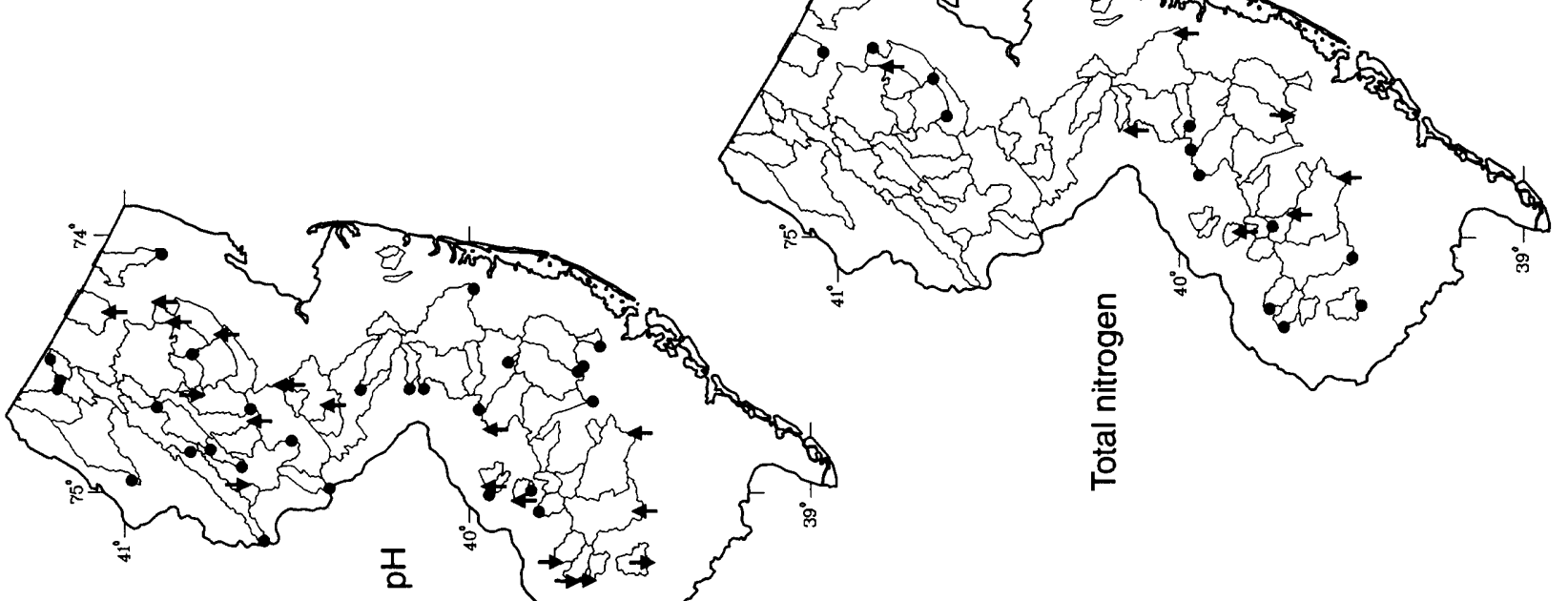

등
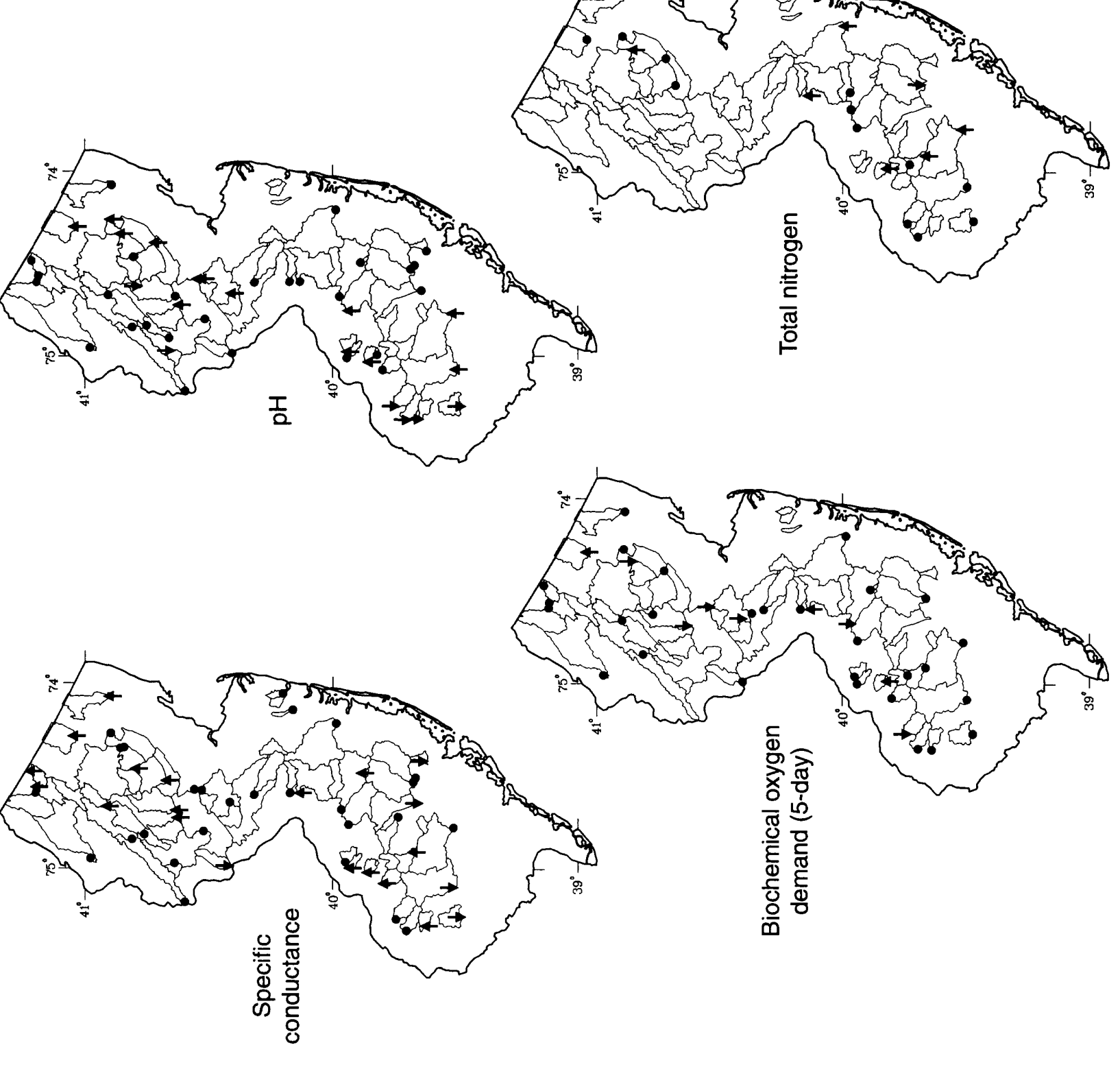


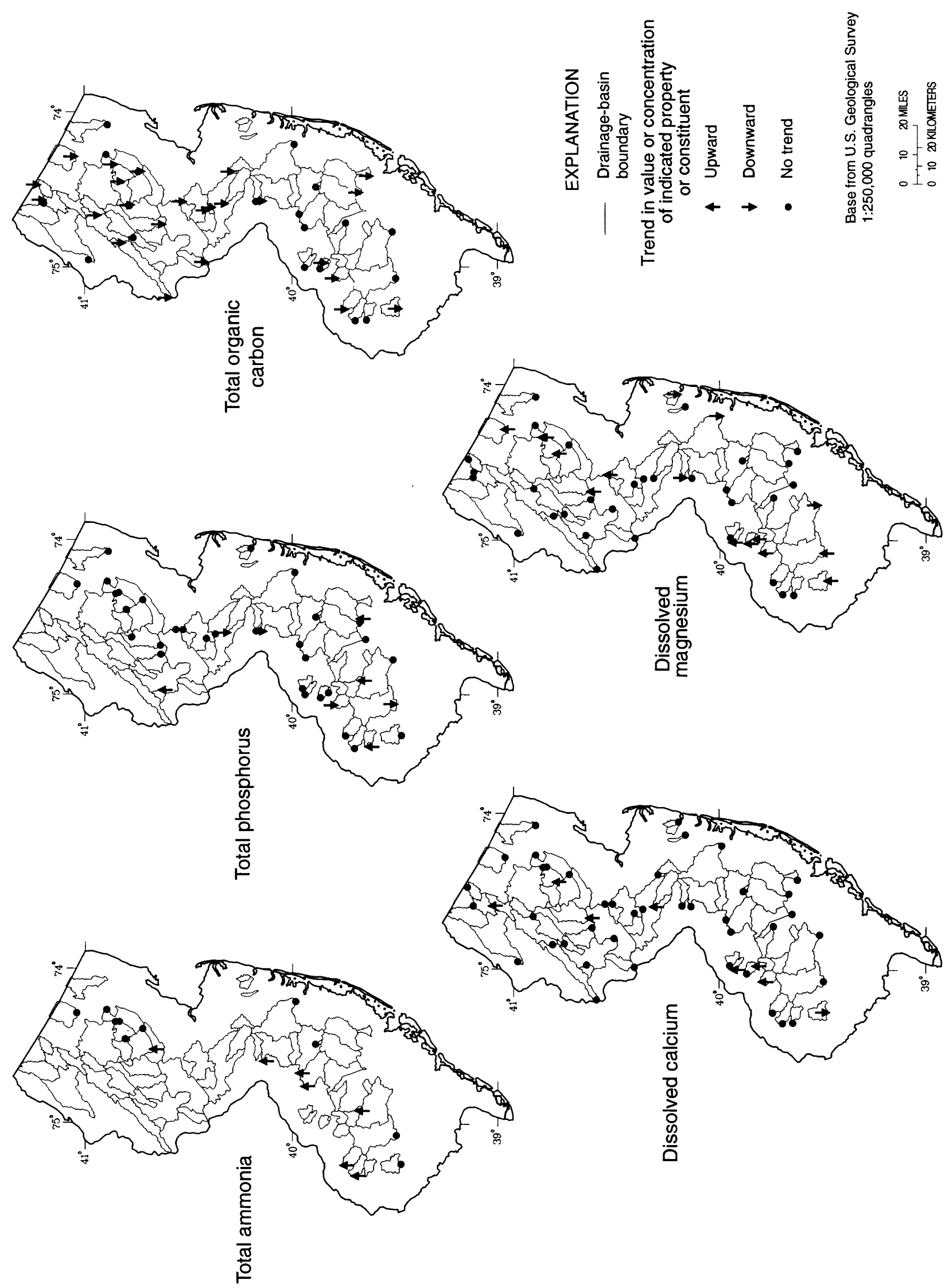

导

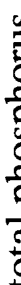

ơ

4

苛 


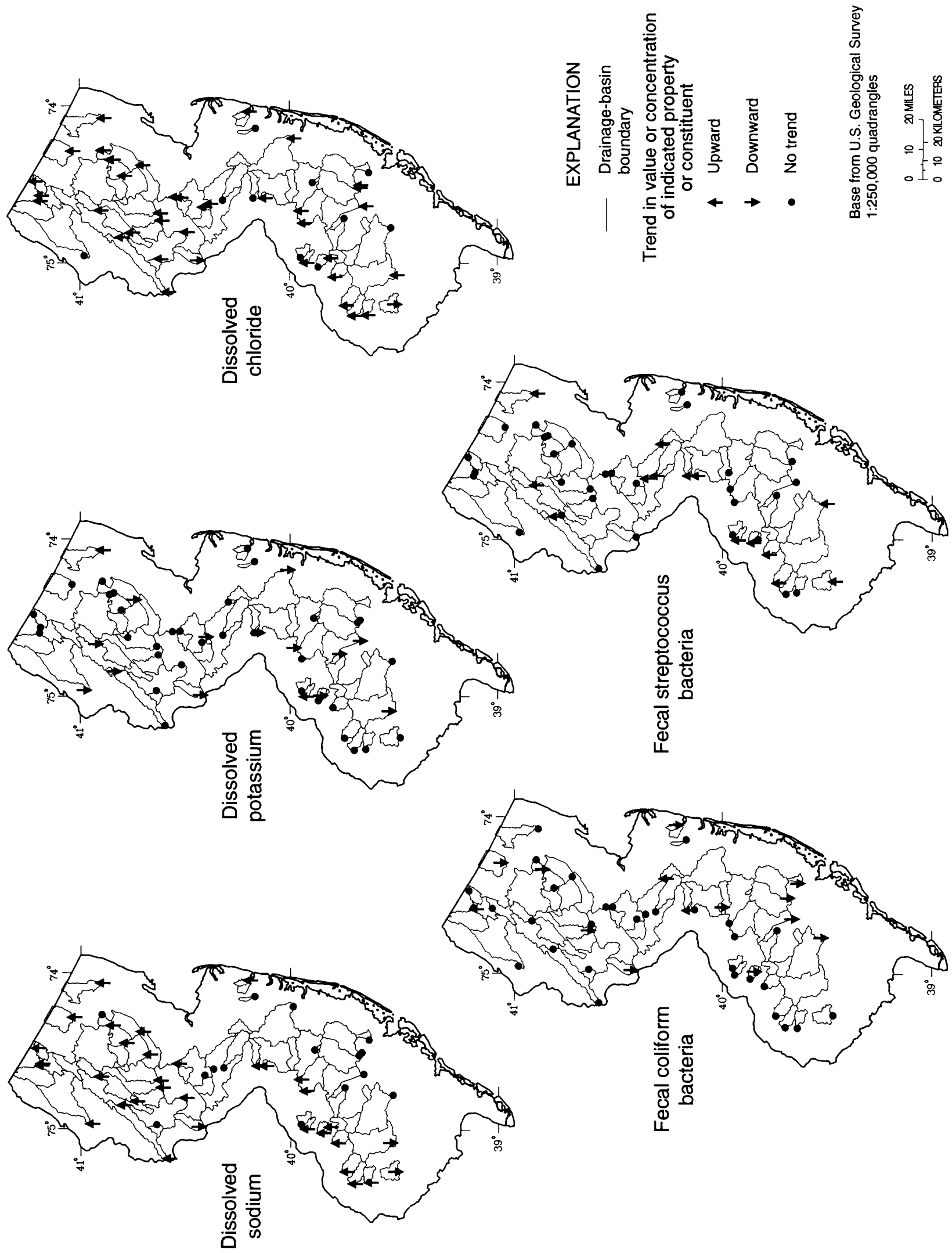


Table 24. Streamwater constituents and properties whose trends were significantly associated with drainage-basin characteristics in New Jersey

Drainage-basin characteristic
Associated trends in constituent concentrations or physical property
1. Drainage-basin size

2. Physiographic region of drainage basin

3. Presence of agricultural, urban, or forest land as the dominant land use
a. Entire drainage basin
b. Area 3.1 miles upstream from monitoring station
c. Area 0.62 mile upstream from monitoring station

4. Population
a. 1986 drainage-basin population
b. 1986 drainage-basin population density
c. Change in population density, 1975-86

5. Wastewater disposal

a. Change in number of wastewater discharges, 1975-86

b. Wastewater yields in entire drainage basin

1. Wastewater discharge bacteria

2. Biochemical oxygen demand, 5-day

3. Total suspended solids

c. Wastewater yields in area 3.1 miles upstream from monitoring station

1. Wastewater discharge

2. Biochemical oxygen demand, 5-day

3. Total suspended solids

d. Percentage of yields in entire basin originating from municipal facilities

1. Wastewater discharge

2. Biochemical oxygen demand, 5-day

3. Total suspended solids

e. Percentage of yields in area 3.1 miles upstream from monitoring station originating from municipal facilities

1. Wastewater discharge

2. Biochemical oxygen demand, 5-day

3. Total suspended solids

6. Road-salt application

a. Trends in sodium use, 1975-86

b. Trends in chloride use, 1975-86

c. Road-salt application (winter 1980)

\section{Chloride}

2. Sodium

3. Calcium

7. Agricultural Activities
a. Trends in phosphorous fertilizer application, 1975-85
b. 1980 applications of phosphorous and nitrogen fertilizers
c. Soil erosion rates
d. Percentage of drainage basin area irrigated

Specific conductance, dissolved chloride

Total organic carbon, dissolved sodium

$\mathrm{pH}$, dissolved potassium, fecal streptococcus bacteria

$\mathrm{pH}$, total ammonia, dissolved magnesium, dissolved potassium, fecal coliform bacteria

$\mathrm{pH}$, total ammonia, dissolved magnesium, dissolved sodium, dissolved chloride

$\mathrm{pH}$

Specific conductance, $\mathrm{pH}$

Biochemical oxygen demand

Specific conductance, total ammonia

$\mathrm{pH}$, dissolved sodium, fecal streptococcus

$\mathrm{pH}$, dissolved sodium

$\mathrm{pH}$, dissolved sodium

Total ammonia, as $\mathrm{N}$

Dissolved calcium, dissolved sodium, dissolved chloride, fecal coliform bacteria

Dissolved calcium, dissolved chloride

Dissolved oxygen

Specific conductance

Dissolved chloride, fecal streptococcus bacteria

Dissolved oxygen, fecal coliform bacteria

Dissolved oxygen

Dissolved oxygen

Dissolved magnesium

Dissolved magnesium

Specific conductance, dissolved magnesium, dissolved sodium, dissolved chloride

Specific conductance, $\mathrm{pH}$

Specific conductance, $\mathrm{pH}$

Dissolved magnesium

Specific conductance, $\mathrm{pH}$

Fecal streptococcus bacteria

$\mathrm{pH}$, dissolved magnesium 
contingency-table analyses by drainage-basin characteristic is given in the paragraphs that follow. Probabilities of statistically strong associations $(p \leq 0.01)$ are noted in the text, as are the probabilities for tests that were nearly statistically significant $(p>0.10$ and $p<0.15)$.

Contingency-table testing for associations among the water-quality trends revealed a number of expected associations (table 25). Increases in the concentration of dissolved ions were frequently associated with one another, as were increases in specific conductance and increases in dissolved ions. Because specific conductance is a measure of electrical conductivity in water, concentrations of dissolved ions are closely related to specific conductance (Hem, 1985). Another expected relation among the trends was an increase in dissolved-oxygen concentration with decreasing biochemical oxygen demand (BOD). Although in-stream dissolved-oxygen concentrations are typically affected by many factors, the amount of oxygendemanding waste (measured as BOD), can be a dominant factor. Certain expected associations between trends, such as between concentrations of dissolved oxygen and nutrients and between fecal coliform and fecal streptococcus bacteria, were not found.

Trends in the concentrations of only one constituent, dissolved sodium, were associated with seasons. Upward trends in dissolved sodium were more frequent during October through January than at other times of the year. Increasing chloride concentrations were nearly associated with seasons $(p=0.146)$ because increases in chloride concentration tended to occur during October through January. These results indicate that basin activities during the fall and winter appear to have a stronger affect on the upward trends in dissolved sodium and chloride concentrations than do activities at other times of the year. Increasing dissolved-oxygen concentrations also were nearly associated with seasons $(p=0.109)$; increasing dissolved oxygen concentrations were present more frequently during August through November than in other months of the year.

\section{Streamflow}

Tests of association between water-quality trends and trends in instantaneous streamflow were done to identify possible effects of changes in streamflow on the observed water-quality changes. These tests also served to measure the effectiveness of the flow-adjustment procedure used by Hay and Campbell (1990).

A statistically significant association between the trends in total nitrogen concentrations and instantaneous-streamflow trends was the only significant association found for all 15 streamwater constituents and properties. Upward trends in this constituent generally occurred where no trends in streamflow were found.

Fecal streptococcus bacteria trends were nearly associated with instantaneousstreamflow trends $(p=0.107)$. Like the total nitrogen trends, upward trends in fecal streptococcus bacteria were detected primarily at stations where no streamflow trends were found. 
Table 25. Significant associations among water-quality trends in the New Jersey drainage basins studied

\begin{tabular}{ll}
\hline $\begin{array}{c}\text { Streamwater property or } \\
\text { constituent with trend }\end{array}$ & \multicolumn{1}{c}{ Associated streamwater property or constituent trends } \\
\hline \hline Specific conductance & $\begin{array}{c}\text { Biochemical oxygen demand, dissolved sodium, dissolved chloride, dissolved } \\
\text { potassium } \\
\text { Biochemical oxygen demand }\end{array}$ \\
Dissolved oxygen & Specific conductance, dissolved oxygen \\
Biochemical oxygen demand & Dissolved calcium \\
pH & -1 \\
Total nitrogen & Dissolved sodium, dissolved magnesium \\
Total ammonia & Fecal streptococcus bacteria \\
Total phosphorus & Dissolved chloride \\
Total organic carbon & pH, dissolved magnesium \\
Dissolved calcium & Dissolved calcium, total ammonia \\
Dissolved magnesium & Dissolved chloride, total ammonia, specific conductance \\
Dissolved sodium & Specific conductance \\
Dissolved potassium & Specific conductance, dissolved sodium, total organic carbon \\
Dissolved chloride & - - \\
Fecal coliform bacteria & Total phosphorus \\
Fecal streptococcus bacteria &
\end{tabular}

${ }^{1}$ No other streamwater-property or -constituent trends associated with trends in this property or constituent. 
The trends determined by Hay and Campbell (1990) for total nitrogen and fecal streptococcus bacteria were, for the most part, not based on flow-adjusted values. As such, associations between trends and streamflow would be expected for these constituents. Overall, the absence of associations between water-quality trends and instantaneous-streamflow trends indicates that the flow-adjustment procedure used by Hay and Campbell (1990) was effective in removing the effects of streamflow variation on the water-quality data.

\section{Geographic Setting}

Most identified trends were uniform throughout the State and were independent of physiographic factors, as evidenced by the absence of associations between the water-quality trends and the physiographic province of the drainage basin. Trends in only two water-quality constituents, downward trends in total organic carbon concentrations and upward trends in dissolved sodium concentrations, were significantly associated with physiographic province. Downward trends in total organic carbon were more common than expected north of the Fall Line. The reasons for this are not known. In contrast, upward trends in dissolved sodium tended to occur at more stations north of the Fall Line ( 70 percent of the drainage basins) than south of the Fall Line ( 52 percent of the drainage basins). The upward trends in dissolved sodium could be due to the more frequent use of road salts in the northern part of the State.

In a second set of tests to determine the distribution of the water-quality trends, associations between the trends and drainage-basin size were evaluated. Overall, the waterquality trends showed little association with drainage-basin size. Only trends in specific conductance and concentrations of dissolved chloride were significantly associated with drainage-basin size. For specific conductance, nearly one-half of the upward trends were found in the smallest drainage basins (smaller than $25.5 \mathrm{mi}^{2}$ ). Increases in dissolved-chloride concentrations were more common at stations in the largest drainage basins (larger than $68.7 \mathrm{mi}^{2}$ ), whereas the smallest drainage basins had a higher number of stations where no trends were found. The conservative chemical behavior of chloride in surface water may explain the frequent increases in the larger drainage basins. This conservative chemical behavior causes chloride concentrations to increase in a downstream direction.

\section{Land Use}

Trends in $\mathrm{pH}$ and several chemical constituents were associated with the dominant land use in the drainage basin. These associations were found at more monitoring stations and were statistically stronger where the dominant land use nearest the monitoring station, as opposed to that of the entire basin, was associated with the trends. In addition to $\mathrm{pH}$, constituents whose trends were associated with land use were concentrations of fecal coliform and streptococcus bacteria, dissolved magnesium, dissolved potassium, dissolved chloride, dissolved sodium, and total ammonia.

Trends in $\mathrm{pH}$ were consistently associated with the dominant land use above the monitoring station, regardless of whether the entire drainage basin or only the areas $3.1 \mathrm{mi}$ and $0.62 \mathrm{mi}$ upstream from the monitoring station were considered. Downward trends in $\mathrm{pH}$ were found at more stations than expected in agricultural basins, whereas upward trends in $\mathrm{pH}$ were 
found at more stations than expected in urban drainage basins. The associations for $\mathrm{pH}$ trends were strongest for the dominant land use in the area $0.62 \mathrm{mi}$ upstream from the monitoring station. These results match information in the literature describing land-use effects on the quality of runoff. Increases in the $\mathrm{pH}$ of runoff from urban drainages have been documented by Halverson and others (1984) and Harrison and Wilson (1985). Dornbush and others (1974) report $\mathrm{pH}$ reductions in runoff from agricultural fields due to the leaching of cations.

Upward trends in the concentrations of dissolved magnesium and potassium were found at more stations than expected in drainage basins dominated by urban land. The association between dissolved-magnesium trends and the dominant land use in the area $0.62 \mathrm{mi}$ upstream from the monitoring station was highly significant $(p=0.002)$. Downward trends in dissolvedpotassium concentrations tended to occur at monitoring stations in forested basins. Trends in the concentrations of two additional dissolved ions, sodium and chloride, were statistically associated with the land use in the area $0.62 \mathrm{mi}$ upstream from the monitoring station and not with the dominant land use in the entire drainage basin. Upward trends in sodium and chloride concentrations during WY 1976-86 were found at monitoring stations where the nearby upstream land use was primarily urban or agriculture. Lettenmaier and others (1991) also reported statistical associations between upward trends in concentrations of dissolved ions and the presence of urban lands.

Trends in the concentrations of fecal streptococcus bacteria were associated with land use in the entire drainage basin, but showed no association with nearby land uses; upward trends were common in agricultural drainage basins. In contrast, fecal coliform bacteria trends were associated with land use only in the area $3.1 \mathrm{mi}$ upstream from the monitoring station. Downward trends in fecal coliform bacteria concentrations were most frequent where the area $3.1 \mathrm{mi}$ upstream was dominated by forest. The ratio of fecal coliform bacteria to fecal streptococcus bacteria is often used to distinguish whether the source of the bacteria is either humans or other warm-blooded animals. In order to determine if the trends in the concentrations of fecal coliform and fecal streptococus bacteria had any association to the fecal coliform/fecal streptococcus ratio, contingency table tests were done with those two variables. No significant associations were detected in the tests; hence, trends in concentrations of these bacteria appear to be independent of each other.

Trends in concentrations of only one of three nutrients were found to be statistically associated with land use. Trends in concentrations of total ammonia were associated with the dominant land use in the areas 0.62 and 3.1 mi upstream from the monitoring station. In these associations, upward trends in concentrations of total ammonia were found at more monitoring stations than expected in agricultural basins; these upward trends were mostly in the southern half of the State. Various forms of ammonia are used in fertilizers to promote plant growth. In a comparison of the quality of streams draining agricultural, urban, and forested lands, Crawford and Lenat (1989) found higher concentrations of dissolved ammonia in the agricultural streams than in streams draining the other land uses. 


\section{Population}

Contingency-table tests that associated water-quality trends with estimates of drainagebasin population and change in population density from 1975 to 1986 identified few statistically significant associations. Only trends in $\mathrm{pH}$, specific conductance, and BOD concentrations were associated with drainage-basin population.

Trends in $\mathrm{pH}$ were strongly associated with estimates of 1986 drainage-basin population $(p=0.007)$ and to population-density estimates. Drainage basins having the lowest estimated population (less than 9,482 persons) and estimated density (less than 303 persons per square mile) had a higher proportion of the $\mathrm{pH}$ downward trends (fig. 14). In contrast, upward trends in $\mathrm{pH}$ occurred more frequently than expected in drainage basins having the greatest estimated population (over 29,781 persons) and density (more than 663 persons per square mile). These associations between $\mathrm{pH}$ trends and population tend to follow the same pattern as the associations found between land use and $\mathrm{pH}$ trends (for example, $\mathrm{pH}$ upward trends in urbanized basins and $\mathrm{pH}$ downward trends in agricultural basins).

Specific conductance trends were associated with drainage-basin population. As with $\mathrm{pH}$ trends, specific conductance downward trends were more common than expected in drainage basins with the lowest population density, whereas upward trends were found where population densities were the greatest.

Estimated change in population density from 1975 to 1986 in the 60 drainage basins was associated with the trends in only one constituent, BOD. Drainage basins with comparatively moderate population growth of 34-70 persons per square mile had the majority of trends in BOD during the 11-year period. A possible cause for the BOD trends in these basins may be the varying ability of existing infrastructure to accommodate the population changes and ultimately treat oxygen-consuming wastes.

\section{Effluent Discharge}

The upgrading of municipal wastewater-treatment systems throughout New Jersey has been considered a major success in water-pollution control in the State (New Jersey Department of Environmental Protection, 1984, 1986). An unanswered question is, "Have these efforts in wastewater management resulted in improved water quality in many of the State's streams?" In a partial attempt to answer this question, the water-quality trends observed by Hay and Campbell (1990) were associated with measures of permitted effluent discharge such as change in the number of permitted effluent discharges during 1975-86, the quality and quantity of reported effluent discharged to streams during 1986-89, and the relative proportion of effluent discharged to streams from municipal facilities and nonmunicipal facilities.

Trends in $\mathrm{pH}$ and in concentrations of dissolved calcium, dissolved sodium, dissolved chloride, and fecal coliform bacteria were most frequently associated with estimates of the quality and quantity of effluent discharged in a drainage basin. Dissolved-oxygen trends were associated with data describing the source of the effluent as either municipal or nonmunicipal. Trends in other streamwater constituents whose presence is frequently related to wastewater- 

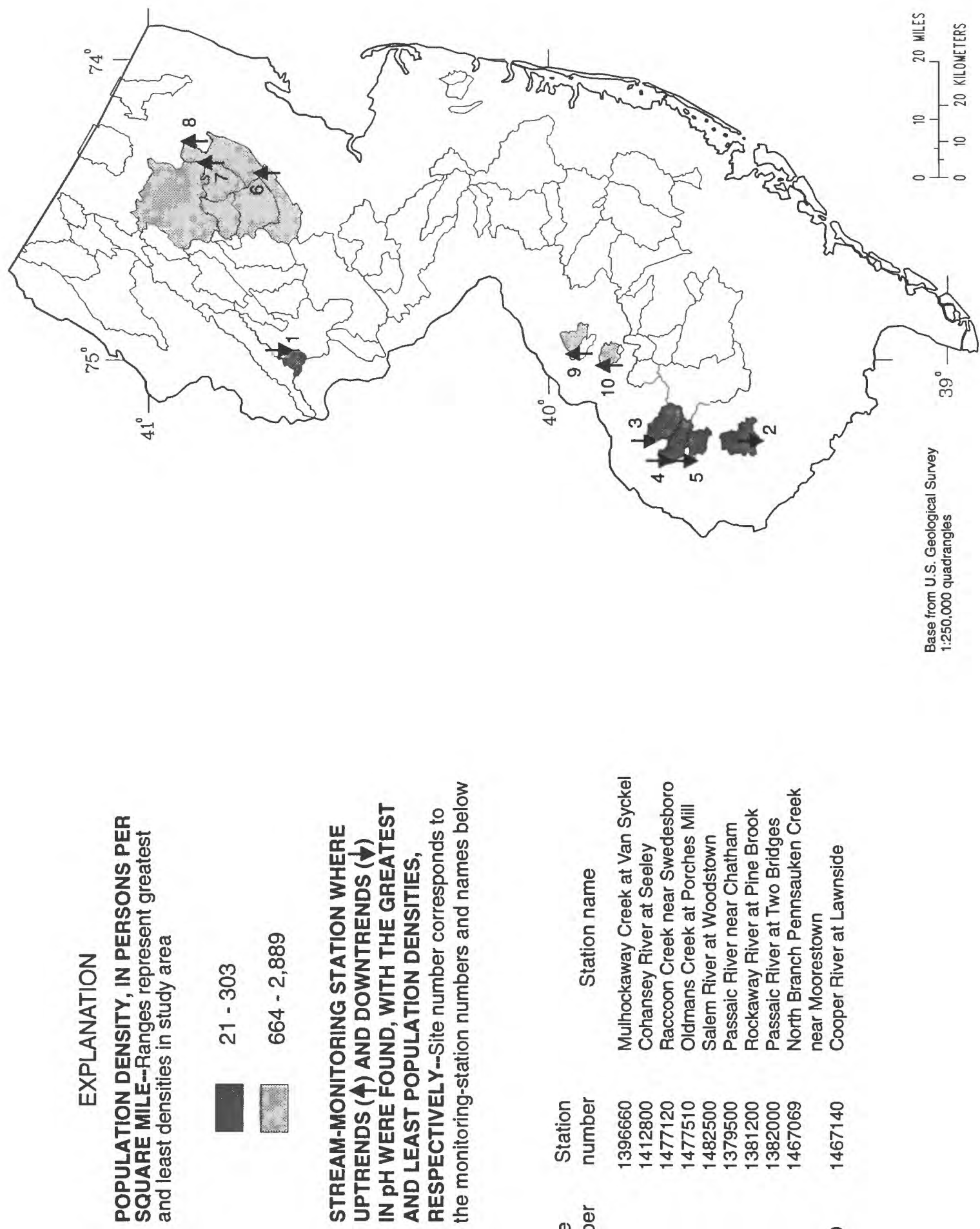

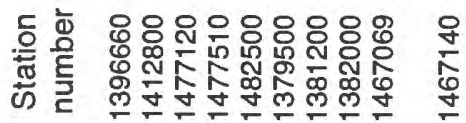
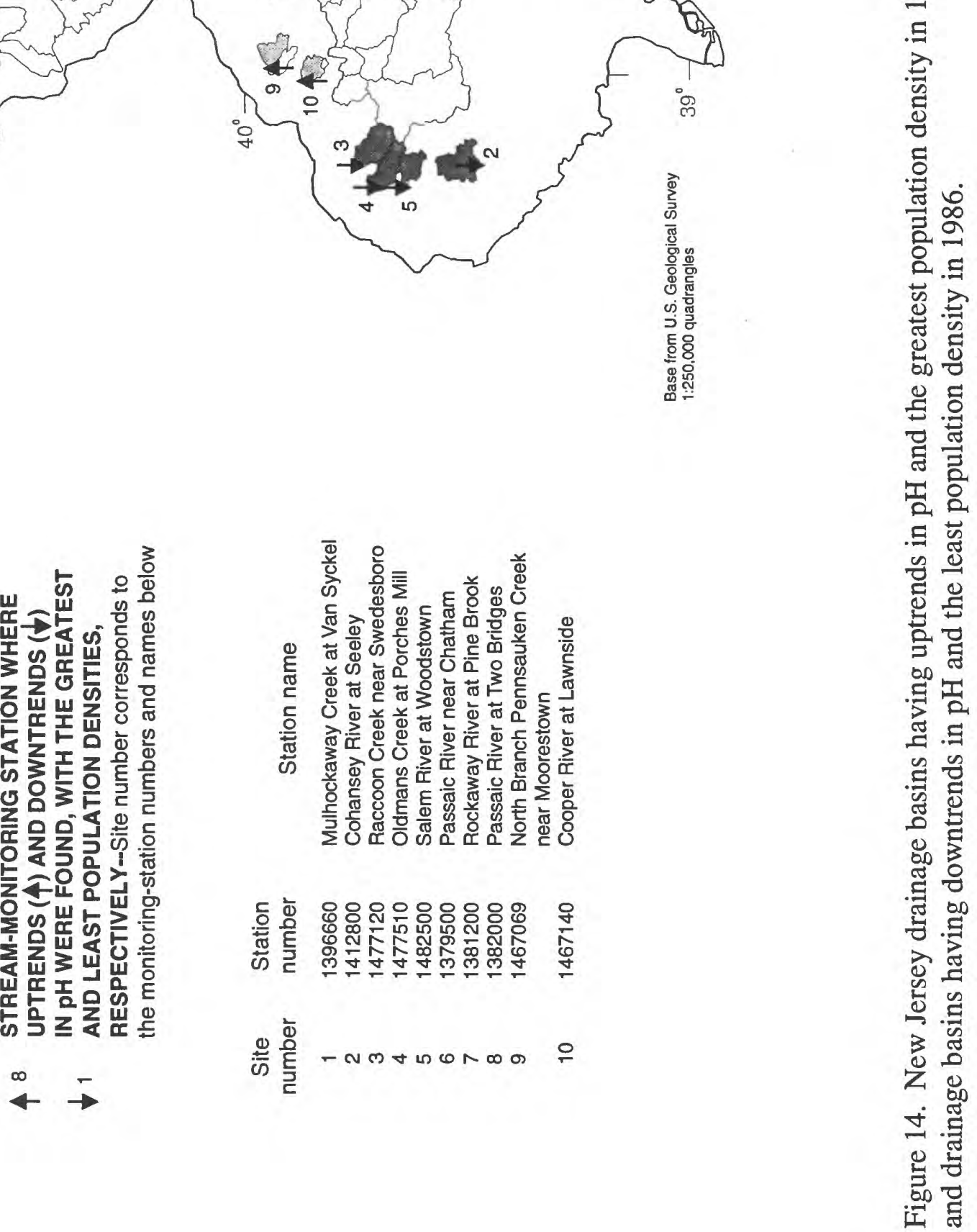
disposal practices (for example, concentrations of $\mathrm{BOD}$, total nitrogen, total organic carbon, and total phosphorus) were found to be minimally related to the measures of effluent discharge used in the analyses. This absence of trends may be indicative of too great a distance between the monitoring station and the zone of assimilation that typically develops below effluent-discharge sites.

Streams in New Jersey where reduced dissolved-oxygen concentrations are most frequent appear to be significantly affected by effluent discharges (New Jersey Department of Environmental Protection, 1990). However, the dissolved-oxygen trends identified by Hay and Campbell (1990) had no statistical association with the yields of effluent or with yields of BOD and TSS discharged upstream from the monitoring station (table 17). Statistical associations were found between the trends in dissolved-oxygen concentrations and information describing the source of effluent in a drainage basin. Upward trends in dissolved-oxygen concentrations were more common than expected in drainage basins where greater than 60 percent of the total effluent was discharged from nonmunicipal treatment facilities (fig. 15). Similar associations were found when the source of the effluent discharge and BOD and TSS loads near the monitoring station (within $3.1 \mathrm{mi}$ ) were compared to the trends in dissolved-oxygen concentrations. These results indicate that nonmunicipal effluent disposal may have had the greater effect on dissolved-oxygen upward trends in the streams. These results are similar to those reported by Smith and others (1987a, 1987b) in their analysis of national water-quality trends, although they tested for trends in dissolved-oxygen deficit rather than dissolved-oxygen concentration. In several water-quality modeling studies for New Jersey streams, the investigators have concluded that instream dissolved-oxygen concentrations will not change significantly for a number of years after improved wastewater treatment at municipal facilities because of the persisting sediment oxygen demand downstream from the facilities (New Jersey Department of Environmental Protection, 1987a, 1987b). This may explain the lack of associations between the trends in dissolved-oxygen concentrations and measures of municipal effluent disposal.

Interpretation of trends in dissolved-oxygen concentrations should also take into account instream temperature change because dissolved-oxygen concentrations vary inversely with temperature. Smith and others $(1987 \mathrm{a}, 1987 \mathrm{~b})$ attempted to reduce the effect of temperature on dissolved-oxygen concentration data by testing for trends in dissolved-oxygen deficit (DOD). The DOD is equal to the dissolved-oxygen-saturation concentration at the time of sample collection minus the observed dissolved-oxygen concentration. Another method for factoring out temperature effects on dissolved oxygen would be to compare trends in dissolved-oxygen concentrations to trends in temperature. Hay and Campbell (1990) detected significant temperature trends at 4 of the 60 monitoring stations. At two of these four monitoring stations, Passaic River at Two Bridges and Raritan River at Manville, concurrent upward trends in temperature and dissolved-oxygen concentrations indicate that the dissolved-oxygen trends may be independent of temperature changes. These two monitoring stations were also below stream reaches where high yields of effluent, BOD, and TSS were discharged.

Trends in $\mathrm{pH}$ were associated with the yield of effluent, BOD, and TSS discharged in the drainage basin; the associations with BOD yields were highly significant $(p=0.006)$.

Monitoring stations where $\mathrm{pH}$ upward trends were found tended to be in drainage basins having 


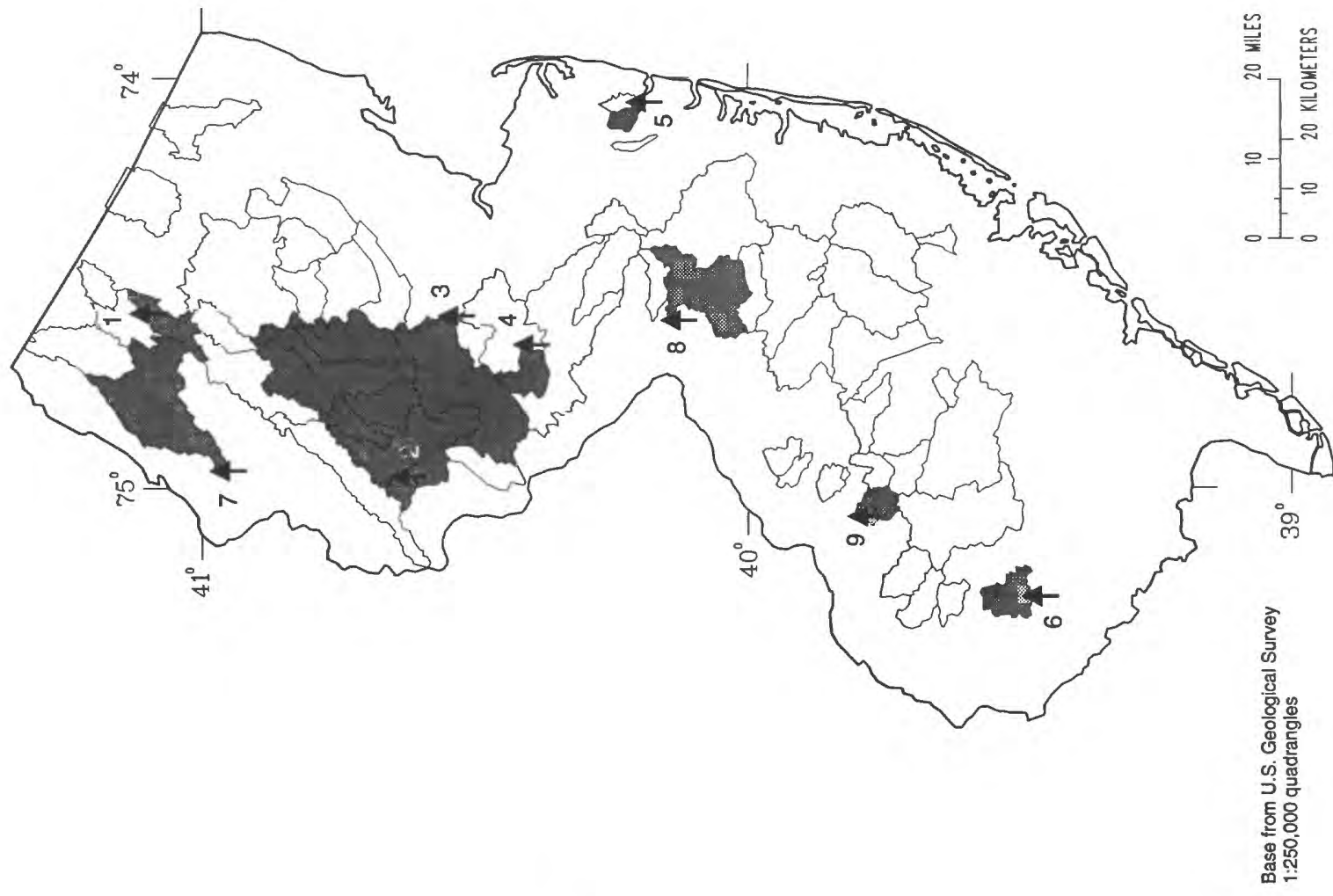

융
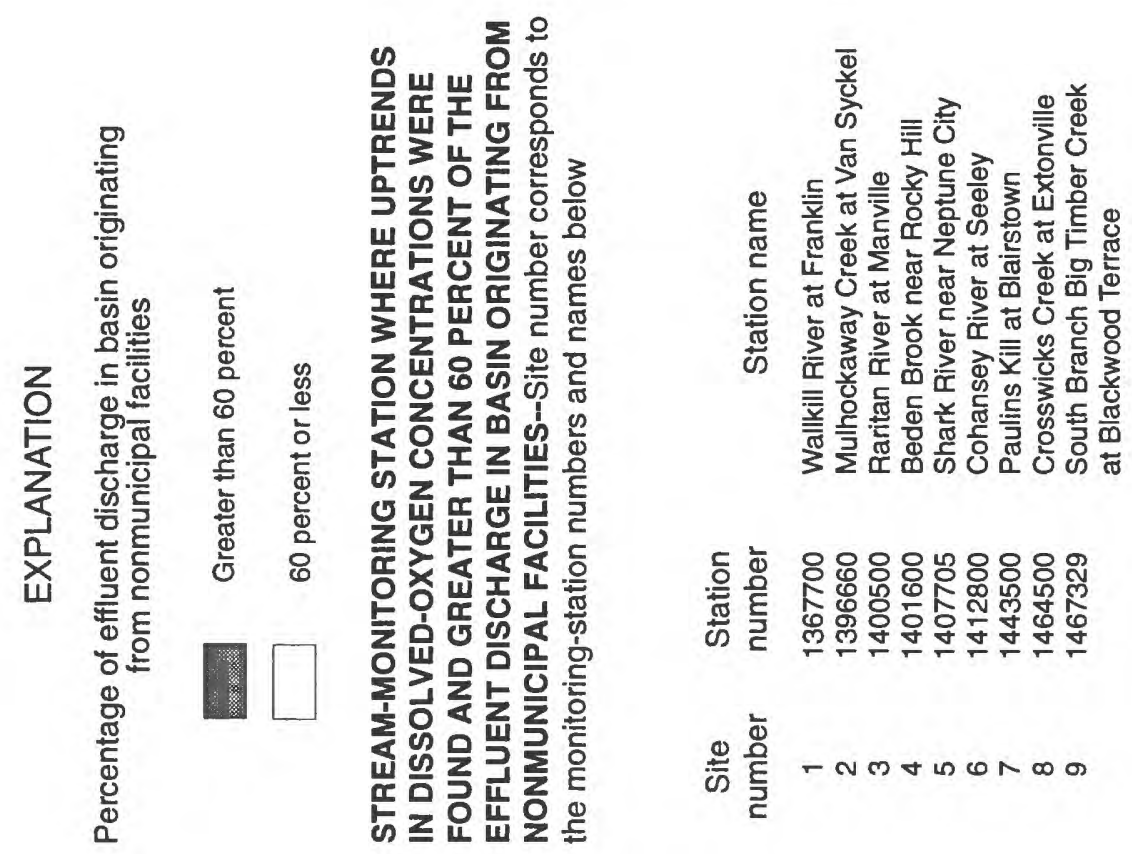

$\infty$

乙。

ஸे

莺 
the greatest yields of these measures of discharged effluent. However, the $\mathrm{pH}$ upward trends were not associated with the primary type of facility (municipal or nonmunicipal) present upstream. Because many of the drainage basins having the greatest yields of effluent also tended to be predominantly urban, a clear pattern is present between $\mathrm{pH}$ increases in the State's waters and urban land use. The investigators hypothesize that the $\mathrm{pH}$ increases are related to stream eutrophication, which in turn leads to increased aquatic-plant growth in the streams. Respiration (photosynthesis) of aquatic plants during daylight hours can cause $\mathrm{pH}$ to rise.

Trends in concentrations of fecal coliform and fecal streptococcus bacteria had statistical associations with various measures of effluent discharge upstream from the monitoring stations. Smith and others $(1987 \mathrm{a}, 1987 \mathrm{~b})$ also reported associations between trends in fecal coliform and streptococcus bacteria and measures of municipal effluent. All downward trends in fecal coliform bacteria concentrations were associated with effluent primarily from municipal sources within the area $3.1 \mathrm{mi}$ upstream from the monitoring stations. Other studies have documented downward trends in fecal bacteria concentrations in streams that received effluent from improved municipal treatment facilities (U.S. General Accounting Office, 1986). Increasing concentrations of fecal streptococcus bacteria had somewhat conflicting associations with measures of effluent discharged. Upward trends in the concentrations of these bacteria were more frequent than expected where effluent was discharged in the drainage basin, as well as where BOD loads in the area $3.1 \mathrm{mi}$ upstream from the monitoring station were smallest (less than $0.08 \mathrm{~kg} / \mathrm{d} / \mathrm{mi} 2$ ). These results indicate that sources other than effluent disposal are the principal influence on fecal streptoccus bacteria concentrations in New Jersey streams. Such possible sources may include waterflow wastes, pet wastes, and urban and agricultural runoff.

Effluent discharged to streams, especially effluent of municipal origin, can contain large quantities of dissolved ions. Upward trends in dissolved calcium, sodium, and chloride in New Jersey streams were frequently associated with measures of effluent discharge. Increases in these dissolved ions were found in drainage basins where yields of effluent discharge were moderate to high. Upward trends in dissolved-chloride concentrations exhibited a statistically strong association with greater amounts of BOD discharged within the area $3.1 \mathrm{mi}$ upstream from the monitoring station $(p=0.006)$. This association may be due to the presence of chloride in sewage and (or) to the chlorination process for disinfecting sewage. Although municipal effluent discharges have been identified as contributing much of the BOD loads in New Jersey during the study period (New Jersey Department of Environmental Protection, 1984), the upward trends in dissolved-chloride concentrations were more prevalent in drainage basins where effluent-related BOD and TSS yields originated from nonmunicipal facilities. Taken together, these associations indicate that permitted effluent discharges and urban land-use activities are likely significant sources of dissolved ions in New Jersey streams. Smith and others $(1987 \mathrm{a}, 1987 \mathrm{~b})$ noted that they expected but did not find statistical associations between trends in dissolved chloride and measures of effluent quantity and quality.

Specific-conductance trends were associated with change in the number of effluent discharges in a drainage basin from 1975 to 1986 and yields of BOD. Upward trends in specific conductance tended to occur at monitoring stations where the number of permitted effluent 
discharges increased and in drainage basins where effluent BOD yields were primarily from municipal facilities. The specific-conductance upward trends may be due to dissolved and suspended ions in the discharged effluent and (or) to the chlorination of wastewaters.

Total ammonia concentration was the only measure of nutrients whose trends had statistical association with measures of effluent discharge. All upward trends in concentrations of total ammonia were in drainage basins having moderate amounts of effluent discharge $(0.003$ $-0.013 \mathrm{Mgal} / \mathrm{day} / \mathrm{square}$ mile). Trends in total phosphorus were not statistically associated with the effluent yield or the source of the effluent, but three of the four upward trends in total phosphorus detected by Hay and Campbell (1990) were in drainage basins where either no effluent or the least amount of effluent was discharged. The overall absence of associations between measures of effluent discharge and nutrient-concentration trends indicate that nonpoint sources may be equal to point sources or of greater importance as a source of nutrients in New Jersey streams. Smith and others (1987a, 1987b) reported strong associations between downward trends in total-phosphorus concentrations and large point-source loads to streams.

\section{Road-Salt Application}

The use of road salt in the 1980-81 winter season was strongly associated with the increased concentrations of dissolved chloride, magnesium, and sodium detected at many monitoring stations on New Jersey streams. Trends in specific conductance also were statistically associated with road-salt applications. However, only trends in dissolvedmagnesium concentrations were statistically associated with trends in the use of chloride or sodium as a road salt during 1975-86. Noteworthy are the absence of associations between instream dissolved-calcium trends and data on calcium use as a deicing material. The dissolvedcalcium trends are, thus, apparently independent of the use of calcium in deicing materials. Most of the loads of calcium in New Jersey's streams probably originate from other sources; road salts may be contributing minor amounts of the total calcium loads.

Trends in concentrations of dissolved chloride and sodium were significantly associated with the estimated 1980-81 winter application of chloride for road deicing. Upward trends in dissolved-chloride and -sodium concentrations were found more frequently than expected in drainage basins having the highest application rates of chloride (fig. 16). Smith and others (1987a, 1987b) also found that increasing dissolved-chloride concentrations were positively correlated with road-salt-application rates. No significant associations were found, however, when dissolved-chloride and -sodium concentration trends were compared to estimated amounts of sodium used for road deicing in 1980 or to trends in chloride and sodium use during 1975-86. As with calcium, other sources or sinks of sodium may mask the detected trends.

A statistically strong association was found between upward trends in dissolvedmagnesium concentrations and drainage basins with the greatest chloride application rates in $1980(p=0.009)$. In addition, upward trends in dissolved-magnesium concentration were strongly associated with trends in chloride and sodium use as road-deicing agents during 1975$86(p=0.002)$. Because magnesium was not identified as being a component in the road salts used by the NJDOT, the increases in dissolved magnesium are probably originating from other sources. The associations with road-salt use are probably coincidental. Dissolved magnesium in 


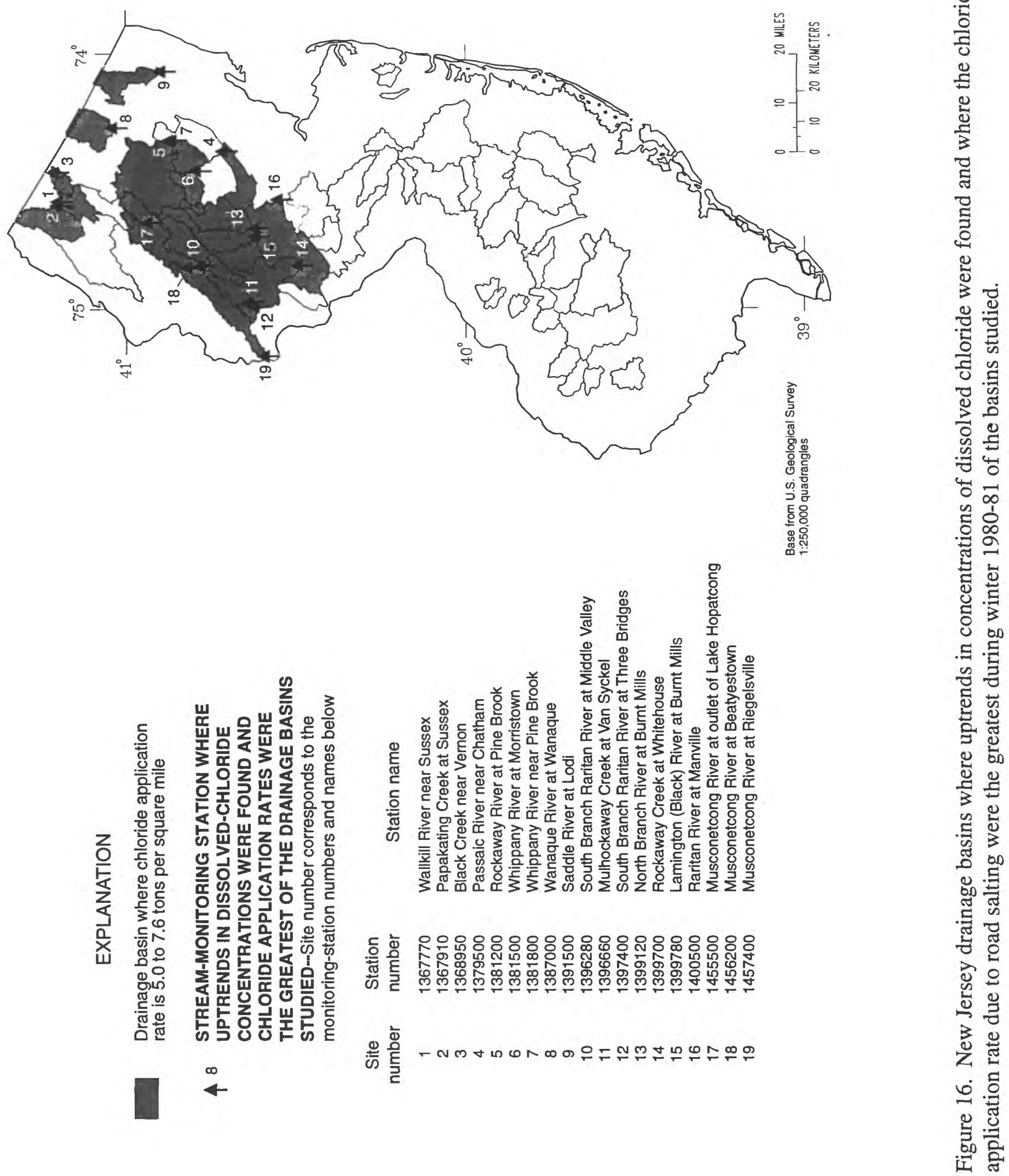


streams has frequently been identified in association with runoff from urban areas, soils, and roadways (Harrison and Wilson, 1985, and Athayde and others, 1983). Tedrow (1986) mentions that magnesium is a common macronutrient in many New Jersey soils and that some soils contain excessive amounts of magnesium. These soils, by way of surface runoff, may be contributing to the increasing dissolved-magnesium concentrations observed. Together, this information points to storm runoff and nonpoint sources as a strong factor in the upward trends in dissolved magnesium.

Statistical associations were found between specific-conductance upward trends and sodium and chloride use in winter 1980-81. Increases in specific conductance were positively associated with drainage basins in which the greatest amounts of sodium and chloride were applied to roadways. Upward trends in specific conductance were nearly associated with drainage basins identified as areas of increasing chloride and sodium use during 1975-86 $(p=0.110)$.

\section{Aaricultural Actlvities}

Four indicators of agriculturally related activities in the 60 drainage basins were compared to the water-quality trends. These indicators were trends in agricultural-fertilizer use during 1975-85 (measured as tons of phosphorus per square mile), amount of agricultural fertilizer applied in 1980 (tons of phosphorus and nitrogen per square mile), soil-erosion rates (tons per acre per year), and percentage of a drainage basin being irrigated (expressed as a percentage of the total acres in the drainage basin). All four of these indicators had few statistically significant associations with the water-quality trends.

Trends in dissolved-magnesium concentrations were associated with the percentage of the area in a drainage basin irrigated and nearly associated with soil-erosion rates. Increases in dissolved-magnesium concentrations were also strongly associated with drainage basins where phosphorus-fertilizer use did not change $(p=0.007)$. Upward trends in dissolved-magnesium concentrations also tended to occur in drainage basins having the greatest percentage of irrigated land. Upward trends in dissolved-magnesium concentrations also were nearly associated with drainage basins where soil-erosion rates were moderate $(5-8$ ton/acre/yr $)(p=0.102)$. The overall strength of associations between dissolved-magnesium upward trends and measures of agricultural activity indicates that either agricultural runoff or soil erosion or both may be contributing factors in the magnesium trends.

Trends in $\mathrm{pH}$ were strongly associated with fertilizer use in the drainage basin $(p=0.009)$ and with the percentage of areas in the basin being irrigated. Upward trends in $\mathrm{pH}$ were found frequently in drainage basins where the greatest amounts of phosphorus and nitrogen fertilizer were applied. Some of this fertilizer is perhaps being transported to area streams through runoff and may be causing eutrophication in those streams. As discussed earlier, increased photosynthesis in streams as a result of eutrophication can contribute to upward trends in $\mathrm{pH}$. Upward trends and downward trends in $\mathrm{pH}$ tended to occur in basins where the highest percentage of land was being irrigated. These $\mathrm{pH}$ trends may be due not only to the release of soil cations during irrigation, which causes $\mathrm{pH}$ to decrease, but also to the flushing of soil nutrients to stream, which results in increased $\mathrm{pH}$ through entrophication. 
Specific-conductance trends were inversely associated with agricultural fertilizer use in 1980. More than one-half of monitoring stations where specific-conductance upward trends were found were in drainage basins where fertilizer-use rates were lowest. Most specificconductance downward trends were found in drainage basins with the highest application rates. These results seem to signify considerable independence between the specific-conductance trends and agricultural activities.

Trends in only one constituent, concentrations of fecal streptococcus bacteria, were found to be significantly associated with soil-erosion estimates, but this association was strong $(p=0.004)$. Drainage basins where cropland soil-erosion rates were moderate (5-8 ton/acre/yr) were associated with upward trends in fecal-streptococcus-bacteria concentrations; these associations were found across the southern part of the State (fig. 17). The increases in concentrations of fecal streptococcus bacteria in basins having moderate soil-erosion rates indicate that agricultural activities are influencing the concentration of this constituent in streamwater.

Upward trends in total phosphorus concentrations were nearly associated with soilerosion rates $(p=0.126)$. These upward trends tended to be more frequent than expected in drainage basins where soil-erosion estimates were greater than 8 ton/acre/yr, an indication that agricultural activities are a source of the increases in total phosphorus concentrations. Smith and others $(1987 \mathrm{a}, 1987 \mathrm{~b})$ reported associations between upward trends in total-phosphorus concentrations throughout the nation and measures of agricultural activities in drainage basins.

\section{SUMMARY AND CONCLUSIONS}

Trends in the concentrations of 13 streamwater constituents and the values of 2 physical properties measured at selected monitoring stations on New Jersey streams during water years 1976-86 (as reported by Hay and Campbell, 1990) were analyzed for statistical associations with various drainage-basin characteristics, including human activities within the basins. These analyses were designed to identify possible causal factors for the water-quality trends. The constituents and properties used in the analyses were $\mathrm{pH}$, specific conductance, and concentrations of dissolved oxygen, biochemical oxygen demand (BOD), total nitrogen, total ammonia, total phosphorus, total organic carbon, dissolved calcium, dissolved magnesium, dissolved sodium, dissolved potassium, dissolved chloride, fecal coliform bacteria, and fecal streptococcus bacteria.

Basin-characteristic data were collected for the drainage basins that were analyzed for trends and whose drainages are entirely or predominantly within New Jersey. Data for some constituents and properties from some monitoring stations on the same streams were excluded from the analysis because correlation among the data violated fundamental analytical assumptions. The number of monitoring stations varied by constituent or property, from 17 for total ammonia to 52 for dissolved sodium.

Information collected on drainage-basin characteristics included measures of physiographic province, area, location, dominant land use, population, effluent discharge, application of road salt, agricultural-fertilizer use, cropland soil-erosion rates, and the amount of 


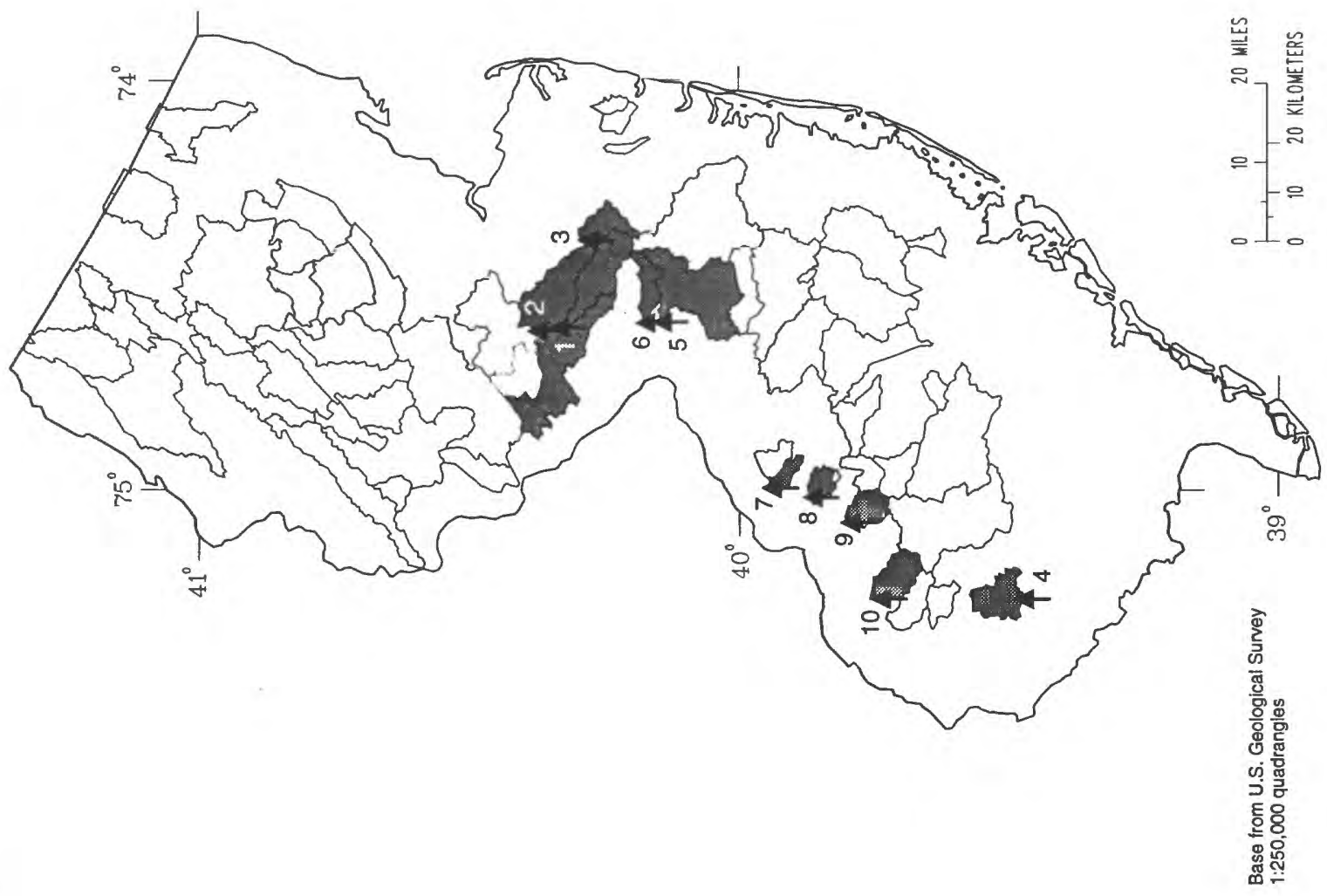

를

选

흐

글

एँ

:

吕

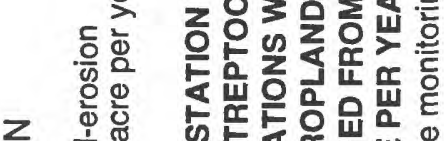

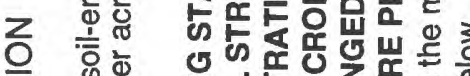

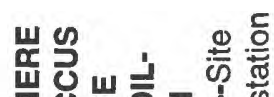
T话可
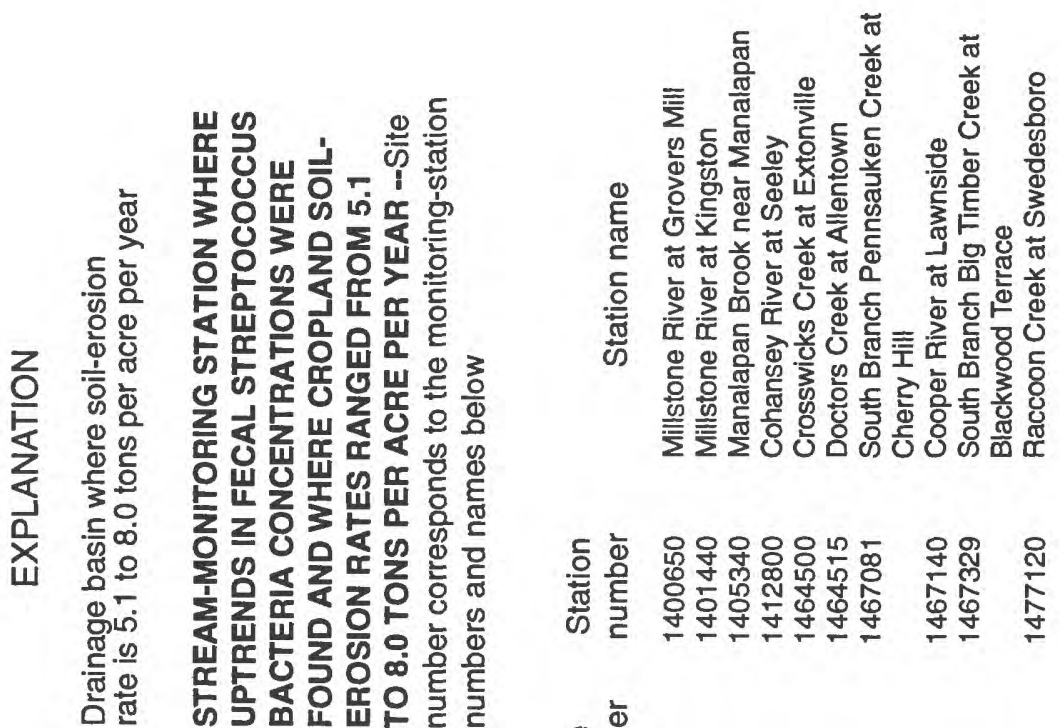

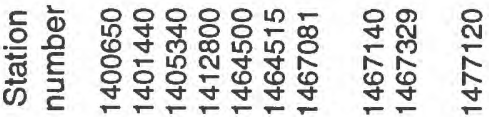

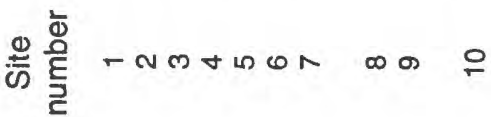

$\infty$ 우

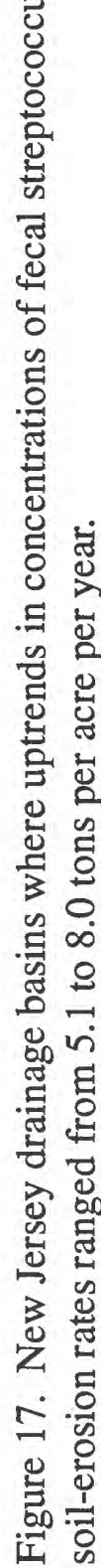


land irrigated for agricultural purposes. Data describing trends during 1975-86 in a particular drainage-basin characteristic were gathered if available, but data were unavailable for most of the characteristics for the full range of years. Otherwise, the characteristic was described for a shorter period within that range. Detailed information on nonpoint sources and atmospheric deposition, two sources suspected as major contributors of dissolved constituents in streamwater, was unavailable for the 60 drainage basins in New Jersey.

Statistical associations between the water-quality trends and drainage-basin characteristics were determined by the use of contingency-table analyses. The contingency-table analyses were also used to identify associations among the water-quality trends and the seasonal occurrence of trends. A significant association was identified at $\alpha=0.10$.

Trends in the concentrations of dissolved constituents were commonly associated with one another, as were the trends in specific conductance with trends in concentrations of dissolved constituents. Another notable association among trend results was upward trends in dissolved-oxygen concentrations with downward trends in BOD concentrations. Most trends in constituent concentrations were associated with trends in the concentration of one or more other constituents.

Most trends in water-quality data were found to be independent of seasons; however, trends in concentrations of dissolved sodium and dissolved oxygen did show seasonal effects. Increases in dissolved-oxygen concentrations were nearly significantly associated with increases in concentrations during late summer and early fall, whereas increases in dissolved-sodium concentrations were associated with increases in dissolved sodium in late fall and early winter. The trends in water-quality data also showed few associations with trends in instantaneous streamflow, an indication that the flow-adjustment procedures used by Hay and Campbell (1990) prior to their trends testing was effective in removing the effects of streamflow on the water-quality data.

The trends in water quality also appear to be generally independent of the area and location of the upstream drainage basins. Of the few associations found, the most notable was the occurrence of upward trends in dissolved-chloride concentrations in the largest drainage basins. This occurrence tends to match the conservative behavior of this ion in streams that results in larger concentrations in a downstream direction.

Tests for association between the dominant land use in the drainage basins and trends in water-quality data revealed various associations, indicating that land use is a strong influence on many of the observed trends. These associations were strongest for the dominant land use closest to the monitoring stations; thus, for certain constituents, local land uses rather than land use in the entire drainage basin have the greater effects on changes in the data. Upward trends in $\mathrm{pH}$ and concentrations of dissolved potassium, calcium, sodium and chloride were strongly associated to land use in many drainage basins, most commonly at stations in urbanized areas. In agricultural basins, upward trends in concentrations of fecal streptococcus bacteria and total ammonia and downward trends in $\mathrm{pH}$ were common. These associations are consistent with results of other studies relating land use to water-quality conditions and trends. 
Measures of drainage-basin population had few associations with trends in water-quality data. Upward trends in specific conductance were common in basins having the highest population densities in the State, whereas downward trends in $\mathrm{pH}$ occurred in basins having the lowest population densities. These associations are similar to those found between the trends in water-quality data and land use (for example, upward trends in specific conductance in urban drainage basins and downward trends in $\mathrm{pH}$ in agricultural drainage basins).

The number of permitted effluent discharges and their releases of BOD and TSS seems to have had a strong affect on water-quality trends in New Jersey based on the number of significant associations between the two variables, even though most discharge points are more than 3 mi upstream from monitoring stations. Upward trends in $\mathrm{pH}$ and concentrations of dissolved sodium and chloride were associated with basins having the greatest yields of effluent, BOD, and TSS. Trends in fecal coliform and streptococcus bacteria concentrations were associated with various measures of effluent discharge. Downward trends in fecal coliform bacteria were found more commonly at monitoring stations near municipal sources of effluent than at stations not near municipal wastewater-treatment facilities. More dissolved-oxygenconcentration upward trends were found at stations where effluents were of nonmunicipal origin than where effluents were of municipal origin. However, trends in dissolved-oxygen concentrations did not show any association with the yields of effluent discharges in a drainage basin. Few associations were found between trends in instream BOD and nutrient concentrations when compared with measures of effluent discharges. This indicates that nonpoint sources could be equal to point sources or of greater importance as contributors of oxygen-demanding substances and nutrients to surface waters of New Jersey or that the monitoring stations are too far downstream from the effluent discharges for BOD and nutrient concentrations to be affected by the discharges.

The prevalence of upward trends in dissolved sodium and chloride concentrations at similar monitoring stations in the State indicates a possible common source of these constituents. One such source is the use of road salt, which increased during the WY 1976-86 period of trend analysis. Positive and commonly strong associations were found between upward trends in concentrations of these dissolved ions at monitoring stations and the amount of sodium and chloride used for road salting upstream; thus, road salting appears to be a significant source of these dissolved ions.

Upward trends in dissolved-magnesium concentrations also were strongly associated with road-salt use. In addition, upward trends in dissolved-magnesium concentrations were strongly associated with urban land and agricultural-fertilizer use. Because magnesium is not a major component of road salts, the cause of the increases in this dissolved ion could be impervious-surface runoff, soil-disturbing activities, and (or) atmospheric deposition.

Upward trends in specific conductance and $\mathrm{pH}$ were found in drainage basins having the greatest amounts of agricultural phosphorus- and nitrogen-fertilizer use in the State. Upward trends in fecal streptococcus bacteria concentration were found in drainage basins where cropland soil-erosion rates were moderate, whereas upward trends in total phosphorus concentrations were nearly associated with the greatest soil-erosion rates in the State. Upward 
trends in $\mathrm{pH}$ and dissolved-magnesium concentrations were found where the amount of land being irrigated was largest. These associations indicate that nonpoint sources could be important factors in the detected trends in the water-quality data.

The downward trends in concentrations of total organic carbon found at monitoring stations throughout New Jersey had very few associations with basin characteristics. This absence of associations indicates that possible causes of the downward trends were not addressed in this report and that further analysis of the trends would be necessary to identify possible causes.

Some of the associations identified between water-quality trends and drainage-basin characteristics in this study are consistent with those reported in earlier studies; these include upward trends in dissolved chloride and sodium in drainage basins having the greatest road-salt applications and upward trends in dissolved-oxygen concentrations where nonmunicipal effluent discharges predominate. Other associations described by those investigators were not found in New Jersey, such as decreases in total phosphorus in drainage basins receiving the greatest amounts of effluent. In addition, many of the associations presented in this report are supported by the results of other studies that more intensely assessed the effects of land use and drainagebasin characteristics on water quality. The absence of associations in New Jersey between trends in nutrients and measures of stream oxygen with basin characteristics indicates that more intensive analysis would be required to identify possible causes for these trends. 


\section{REFERENCES CITED}

Alexander, R.B., and Smith, R.A., 1990, County-level estimates of nitrogen and phosphorus fertilizer use in the United States, 1945 to 1985: U.S. Gcological Survey Open-File Report 90-130, $12 \mathrm{p}$.

Anderson, J.R., Hardy, E.E., Roach, J.T., and Witmer, R.E., 1976, A land use and land cover classification system for use with remote sensor data: U.S. Geological Survey Professional Paper 964, 28 p.

Athayde, D.N., Shelley, P.E., Driscoll, E.D., Gaboury, D., and Boyd, G., 1983, Results of the nationwide urban runoff program, volume 1-final report: U.S. Environmental Protection Agency, Office of Water Program Operations, Washington, D.C., 200 p.

Association of State and Interstate Water Pollution Control Administrators (ASIWPCA), 1984, America's clean water: The State's evaluation of progress: 1972-1982; Washington, D.C., $17 \mathrm{p}$.

Ayers, M.A., and Pustay, E.A., 1988, New Jersey ground-water quality, in U.S. Geological Survey, National Water Summary 1986--hydrologic events and ground-water quality: U.S. Geological Survey Water-Supply Paper 2325, p. 369-376.

Bauersfeld, W.R., Schopp, R.D., and Shulman, M.D., 1991, New Jersey floods and droughts, in U.S. Geological Survey, National water summary 1988-89-- hydrologic events and floods and droughts: U.S. Geological Survey Water-Supply Paper 2375, p. 401-408.

Cochran, W.G., 1952, The $\mathrm{x}^{2}$ test of goodness-of-fit: Annals of Mathematical Statistics, v. 23, p. 315-345.

Cohn, T.A., and Stedinger, J.R., 1987, Use of historical information in a maximum-likelihood framework: Journal of Hydrology, v. 96, p. 215-223.

Conover, W.J., 1980, Practical nonparametric statistics (2d ed.): New York, Wiley and Sons, $493 \mathrm{p}$.

Crawford, C.G., Slack, J.R., and Hirsch, R.M., 1983, Nonparametric tests for trends in waterquality data using the Statistical Analysis System: U.S. Geological Survey Open-File Report 83-550, $102 \mathrm{p}$.

Crawford, J.K., and Lenat, D.R., 1989, Effects of land use on the water quality and biota of three streams in the Piedmont Province of North Carolina: U.S. Geological Survey WaterResources Investigations Report 89-4007, 67 p. 


\section{REFERENCES CITED--Continued}

Dornbush, J.N., Andersen, J.R., and Harmes, L.L., 1974, Quantification of pollutants in agricultural runoff: Washington, D.C., U.S. Environmental Protection Agency, EPA-660/ 2-74-005, $149 \mathrm{p}$.

Halverson, H.G., DeWalle, D.R., and Sharpe, W.E., 1984, Contribution of precipitation to quality of urban storm runoff: Water Resources Bulletin, v. 20, p. 859-864.

Harrison, R.M., and Wilson, S.J., 1985, Chemical composition of highway drainage waters-I. major ions and selected trace metals: Science of the Total Environment, v. 43, p. 63-77.

Hay, L.E., and Campbell, J.P, 1990, Water-quality trends in New Jersey streams: U.S. Geological Survey Water-Resources Investigations Report 90-4046, 297 p.

Hem, J.D., 1985, Study and interpretation of the chemical characteristics of natural water (3d ed.): U.S. Geological Survey Water-Supply Paper 2254, 263 p.

Hirsch, R.M., Slack, J.R., and Smith, R.A., 1982, Techniques of trend analysis for monthly water quality data: Water Resources Research, v. 18, p. 107-121.

Hirsch, R.M., and Slack, J.R., 1984, A nonparametric test for seasonal data with serial dependence: Water Resources Research, v. 20, p. 727-732.

Lettenmaier, D.P., Hooper, E.R., Wagoner, Colin, and Faris, K.B., 1991, Trends in stream quality in the continental United States, 1978-87: Water Resources Research, v. 27, p. 327-339.

Ludlum, D.M., 1983, The New Jersey weather book: New Brunswick, N.J., Rutgers University Press, 249 p.

Mitchell, W.B., Guptill, S.C., Anderson, K.E., Fegeas, R.C., and Hallam, C.A., 1977, GIRAS-geographic information retrieval and analysis system for handling land use and land cover data: U.S. Geological Survey Professional Paper 1059, 16 p.

Morgan, K.O., Morgan, Scott, and Quitno, Neal, eds., 1992, State rankings, 1992--a statistical view of the 50 United States: Lawrence, Kans., Morgan Quitno Corporation, 459 p.

New Jersey Department of Agriculture, 1989, New Jersey agriculture 1989 annual report-agricultural statistics: Trenton, N.J., Circular 524, 86 p.

New Jersey Department of Environmental Protection, 1984, New Jersey 1984 state water quality inventory report--report on the status of water quality in New Jersey pursuant to the New Jersey Water Pollution Control Act and Section 305(b) of the Federal Clean Water Act: Trenton, N.J., $116 \mathrm{p}$. 


\section{REFERENCES CITED--Continued}

1985, Environmental information inventory--Division of Water Resources: Trenton, N.J., variously paginated.

1986, New Jersey 1986 state water quality inventory report--report on the status of water quality in New Jersey pursuant to the New Jersey Water Pollution Control Act and Section 305(b) of the Federal Clean Water Act: Trenton, N.J., 298 p.

1987a, Passaic River water quality management study: Trenton, N.J., Division of Water Resources, special report, $249 \mathrm{p}$.

1987b, Upper Millstone River water quality management study: Trenton, N.J., Division of Water Resources, special report, $148 \mathrm{p}$.

1988, New Jersey 1988 state water quality inventory report--report on the status of water quality in New Jersey pursuant to the New Jersey Water Pollution Control Act and Section 305(b) of the Federal Clean Water Act: Trenton, N.J., 360 p.

1990, New Jersey 1990 state water quality inventory report--report on the status of water quality in New Jersey pursuant to the New Jersey Water Pollution Control Act and Section 305(b) of the Federal Clean Water Act: Trenton, N.J., 471 p.

New Jersey Department of Labor, 1976, Population estimates for New Jersey-- July 1, 1976: Trenton, N.J., 42 p.

1987, Population estimates for New Jersey--July 1, 1986: Trenton, N.J., 40 p.

Robinson, K.W., Price, C.V., and Smith, R.A., 1995, Development of a computerized data base of permitted wastewater discharges in New Jersey: U.S. Geological Survey Open-File Report 95-152, $14 \mathrm{p}$.

Scott, W.S., 1980, Road salt movement in two Toronto streams: Journal of the Environmental Engineering Division, American Society of Civil Engineers, v. 106, p. 547-560.

Seaber, P.R., Kapinos, F.P., and Knapp, G.L., 1987, Hydrologic unit maps: U.S. Geological Survey Water-Supply Paper 2294, 63 p.

Smith, R.A., Alexander, R.B., and Wolman, M.G., 1987a, Analysis and interpretation of waterquality trends in major U.S. rivers, 1974-81: U.S. Geological Survey Water Supply Paper $2307,25 \mathrm{p}$.

1987b, Water-quality trends in the Nation's rivers: Science, v. 235, p. 1607-1615. 


\section{REFERENCES CITED--Continued}

Snedecor, G.W., and Cochran, W.G., 1967, Statistical methods (6th ed.): Ames, Iowa, The Iowa State University Press, 593 p.

Tedrow, J.C.F., 1986, Soils of New Jersey: Malabar, Fla., Robert E. Krieger Publishing Company, $479 \mathrm{p}$.

U.S. Department of Agriculture, 1986, The state erosion, sediment, and animal waste (SESAW) study--New Jersey: Somerset, N.J., 59 p.

U.S. Department of Commerce, 1991, State and metropolitan area data book, 1991, metropolitan areas, central cities, states--a statistical abstract supplement: Washington, D.C., Bureau of the Census, $388 \mathrm{p}$.

U.S. Environmental Protection Agency, 1984, Report to Congress--nonpoint source pollution in the U.S.: Washington, D.C., 97 p., 3 app.

1987, National water quality inventory--1986 report to Congress: Washington, D.C., 141 p., 1 app.

1990a, National water quality inventory--1988 report to Congress: Washington, D.C., 189 p., 1 app.

1990b, Office of Water--environmental and program information systems compendium-FY 1990: U.S. Environmental Protection Agency, EPA 500/9-90-002, 125 p.

U.S. General Accounting Office, 1986, An evaluation method for the construction grants program--methodology: Washington, D.C., Report to the Administrator, Environmental Protection Agency, GAO/PEMD-87-4A, 82 p.

U.S. Geological Survey, 1990, National water summary 1987--Hydrologic events and water supply and use: U.S. Geological Survey Water-Supply Paper 2350, 553 p.

U.S. Office of Management and Budget, 1987, Standard industrial classification manual--1987:

Washington, D.C., Executive Office of the President, 705 p.

van Belle, Gerald, and Hughes, J.P., 1984, Nonparametric tests for trend in water quality: Water Resources Research, v. 20, p. 127-136.

Velnich, A.J., 1982, Drainage areas in New Jersey--Delaware River Basin and streams tributary to Delaware Bay: U.S. Geological Survey Open-File Report 82-572, 33 p.

1984, Drainage areas in New Jersey--Atlantic coastal basins, South Amboy to Cape May: U.S. Geological Survey Open-File Report 84-150, 48 p. 
Table 9. Permitted effluent discharges in the New Jersey drainage basins studied, 1975

[NJPDES, New Jersey Pollutant Discharge Elimination System; --, no name of permitted discharge found. Source: Data from Michael Dillon, New Jersey Department of Environmental Protection, written commun., 1989]

\begin{tabular}{|c|c|c|c|}
\hline \multicolumn{2}{|c|}{ Drainage basin } & \multirow{2}{*}{$\begin{array}{c}\text { NJPDES } \\
\text { permit } \\
\text { number }\end{array}$} & \multirow[b]{2}{*}{ Permitted effluent discharger } \\
\hline Monitoring-station name & $\begin{array}{l}\text { Monitoring- } \\
\text { station number }\end{array}$ & & \\
\hline \multirow[t]{2}{*}{ Wallkill River at Franklin } & 01367700 & 4596 & THE NEW JERSEY ZINC COMPANY \\
\hline & & 33472 & TRI-COUN'TY WATER CONDITIONING CO. \\
\hline \multirow[t]{9}{*}{ Wallkill River near Sussex } & 01367770 & 141 & AMES RUBBER CORPORATION \\
\hline & & 2275 & ACCURATE FORMING CORPORATION \\
\hline & & 4596 & THE NEW JERSEY ZINC COMPANY \\
\hline & & 6661 & PLASTOID CORPORATION \\
\hline & & 20885 & PLASTOID CORPORATION \\
\hline & & 22055 & FRANKLIN, BD. OF PUBLIC WORKS, BOROUGH \\
\hline & & 27367 & WALLKILL SEWER COMPANY \\
\hline & & 31038 & FRANKLIN BD. OF PUBLIC WORKS \\
\hline & & 33472 & TRI-COUN'TY WATER CONDITIONING CO. \\
\hline Papakating Creek at Sussex & 01367910 & 29041 & REGENCY APARTMENTS \\
\hline \multirow[t]{3}{*}{ Black Creek near Vernon } & 01368950 & 21814 & VERNON VALLEY RECREATION ASSN \\
\hline & & 23841 & VERNON TWP. SCHOOL BOARD \\
\hline & & 23949 & GREAT GORGE'S MOUNTAIN VIEW \\
\hline \multirow[t]{2}{*}{ Passaic River near Millington } & 01379000 & 2925 & MILLINGTON QUARRY \\
\hline & & 29912 & NEW JERSEY DEPT. OF TRANSPORTATION \\
\hline \multirow[t]{16}{*}{ Passaic River near Chatham } & 01379500 & 442 & AT\&T BELL LABORATORIES \\
\hline & & 2551 & REHEIS CHEMICAL CO. \\
\hline & & 2607 & PLASMA GRAPHICS CORPORATION \\
\hline & & 2925 & -- \\
\hline & & 3042 & MILLMASTER CHEMICAL COMPANY \\
\hline & & 3140 & AZOPLATE CORPORATION \\
\hline & & 20281 & PARK CENTRAL SEWAGE TREATMENT \\
\hline & & 21083 & VETERANS ADMINISTRATION \\
\hline & & 21636 & NEW PROVIDENCE BOROUGH OF WTP \\
\hline & & 22489 & WARREN TWP. SEWAGE AUTHORITY \\
\hline & & 22497 & STAGE IV SEWAGE TREATMENT PLT \\
\hline & & 22845 & HARRISON BROOK \\
\hline & & 24465 & PASSAIC, TOWNSHIP OF \\
\hline & & 27961 & WATER POLLUTION CONTROL \\
\hline & & 29912 & NEW JERSEY DEPT. OF TRANSPORTATION \\
\hline & & 29963 & GLASFLEX CORPORATION \\
\hline \multirow[t]{5}{*}{ Rockaway River at Pine Brook } & 01381200 & 1261 & KEUFFEL \& ESSER CO \\
\hline & & 1635 & HOWMET TURBINE COMPONENTS CORP. \\
\hline & & 2496 & MC WILLIAMS FORGE CO INC \\
\hline & & 2500 & PICATINNY ARSENAL \\
\hline & & 2593 & GHA LOCK JOINT \\
\hline
\end{tabular}


Table 9. Permitted effluent discharges in the New Jersey drainage basins studied, 1975--Continued

\begin{tabular}{|c|c|c|c|}
\hline \multicolumn{2}{|l|}{ Drainage basin } & \multirow{2}{*}{$\begin{array}{c}\text { NJPDES } \\
\text { permit } \\
\text { number }\end{array}$} & \multirow[b]{2}{*}{ Permitted effluent discharger } \\
\hline Monitoring-station name & $\begin{array}{l}\text { Monitoring- } \\
\text { station number }\end{array}$ & & \\
\hline \multirow[t]{22}{*}{ Rockaway River at Pine Brook--Continued } & $01381200-$ Cont. & 3409 & MT HOPE ROCKS PRODUCTS \\
\hline & & 3441 & PYAH INDUSTRIES INC \\
\hline & & 3506 & ADRON \\
\hline & & 3611 & CARPENTER LE \& COMPANY INC \\
\hline & & 21181 & CEDAR HILL SCHOOL STP \\
\hline & & 22349 & ROCKAWAY VALLEY REG S.A. \\
\hline & & 22802 & WHITE MEADOW LAKE PROP.OWNERS \\
\hline & & 24457 & OUR LADY OF THE MAGNIFICAT \\
\hline & & 25712 & VIBRATION MOUNTING \& CONTROLS \\
\hline & & 26115 & HARDEN FUEL / V \& H TRUCKING \\
\hline & & 26603 & RANDOLPH HIGH SCHOOL STP \\
\hline & & 26867 & JEFFERSON TWP.-ROCK \\
\hline & & 27847 & SERVOMETER CORPORATION \\
\hline & & 29394 & BERKSHIRE SAND \& STONE CO INC \\
\hline & & 30287 & MONTVILLE TOWNSHIP MUA \\
\hline & & 30317 & MONTVILE TOWNSHIP MUA \\
\hline & & 30911 & SCERBO BROTHERS INC \\
\hline & & 32166 & ASCO ELECTRIC \\
\hline & & 32221 & LESLIE CO \\
\hline & & 32808 & ROCKAWAY TOWNSQUARE MALL \\
\hline & & 35050 & W P REALTY CO \\
\hline & & 52396 & PNEU HYDRO PRODUCTS INC \\
\hline \multirow[t]{7}{*}{ Whippany River at Morristown } & 01381500 & 2542 & PARKE-DAVIS DIV WARNER LAMBERT \\
\hline & & 24929 & WOODLAND STP \\
\hline & & 25674 & ACTION TECHNOLOGY COMPANY \\
\hline & & 26689 & ENGR. DEPT.-GREYSTONE PARK \\
\hline & & 26751 & ST MARY'S ABBEY \\
\hline & & 29734 & FABRICATED PLASTICS \\
\hline & & 33684 & CHAMPION INTL DAIRYPAK DIV \\
\hline \multirow[t]{10}{*}{ Whippany River near Pine Brook } & 01381800 & 1155 & SANDOZ PHARMACEUTICALS CORPORATION. \\
\hline & & 1708 & SANDOZ PHARMACEUTICALS CORPORATION. \\
\hline & & 1881 & PRECISION ROLLED PRODUCTS INC. \\
\hline & & 2542 & PARKE-DAVIS DIV WARNER LAMBERT \\
\hline & & 3450 & LEEMING PACQUIN COMPANY \\
\hline & & 3476 & EXXON RESEARCH \& ENGRG CO \\
\hline & & 3697 & COLLOID CHEMICAL INC \\
\hline & & 24902 & HANOVER SEWERAGE AUTHORITY \\
\hline & & 24911 & BUTTERWORTH STP \\
\hline & & 24929 & WOODLAND STP \\
\hline
\end{tabular}


Table 9. Permitted effluent discharges in the New Jersey drainage basins studied, 1975--Continued

\begin{tabular}{|c|c|c|c|}
\hline \multicolumn{2}{|l|}{ Drainage basin } & \multirow{2}{*}{$\begin{array}{c}\text { NJPDES } \\
\text { permit } \\
\text { number }\end{array}$} & \multirow[b]{2}{*}{ Permitted effluent discharger } \\
\hline Monitoring-station name & $\begin{array}{l}\text { Monitoring- } \\
\text { station number }\end{array}$ & & \\
\hline \multirow[t]{10}{*}{ Whippany River near Pine Brook--Continued } & 01381800-Cont. & 25496 & MORRISTOWN TOWN OF \\
\hline & & 25674 & ACTION TECHNOLOGY COMPANY \\
\hline & & 25739 & LITTON INDUSTRIES INC AIRTRON \\
\hline & & 26689 & ENGR. DEPT.-GREYSTONE PARK \\
\hline & & 26751 & ST MARY'S ABBEY \\
\hline & & 28339 & CAMPBELL-PRATT OIL CO \\
\hline & & 29734 & FABRICATED PLASTICS \\
\hline & & 33685 & CHAMPION INTL DAIRYPAK DIV \\
\hline & & 35777 & EXXON CENTRAL SERVICES \\
\hline & & 36081 & EAST HANOVER TWP WELL NO2 FLTR \\
\hline \multirow[t]{30}{*}{ Passaic River at Two Bridges } & 01382000 & 442 & AT\&T BELL LABORATORIES \\
\hline & & 540 & CIBA-GEIGY CORPORATION \\
\hline & & 1155 & SANDOZ PHARMACEUTICALS CORP. \\
\hline & & 1261 & KEUFFEL \& ESSER CO \\
\hline & & 1490 & ORANGE PRODUCTS INC \\
\hline & & 1635 & HOWMET TURBINE COMPONENTS CORP. \\
\hline & & 1651 & FRITZSCHE DODGE \& OLCOTT \\
\hline & & 1708 & ROWE INTERNATIONAL OF CANADA \\
\hline & & 1881 & PRECISION ROLLED PRODUCTS INC. \\
\hline & & 2003 & AUTOMATIC SWITCH COMPANY \\
\hline & & 2496 & MC WILLIAMS FORGE CO INC \\
\hline & & 2500 & PICATINNY ARSENAL \\
\hline & & 2542 & PARKE-DAVIS DIV WARNER LAMBERT \\
\hline & & 2551 & REHEIS CHEMICAL CO. \\
\hline & & 2593 & GHA LOCK JOINT \\
\hline & & 2607 & -- \\
\hline & & 2828 & WHIPPANY PAPERBOARD CO. \\
\hline & & 2925 & -- \\
\hline & & 3042 & -- \\
\hline & & 3140 & -- \\
\hline & & 3409 & MT HOPE ROCKS PRODUCTS \\
\hline & & 3441 & -- \\
\hline & & 3450 & LEEMING PACQUIN COMPANY \\
\hline & & 3476 & EXXON RESEARCH \& ENGRG CO \\
\hline & & 3506 & ADRON \\
\hline & & 3611 & CARPENTER LE \& COMPANY INC \\
\hline & & 3620 & W F \& JOHN BARNES \\
\hline & & 3697 & COLLOID CHEMICAL INC \\
\hline & & 3743 & M.POLNAR INC. \\
\hline & & 20281 & PARK CENTRAL SEWAGE TREATMENT \\
\hline
\end{tabular}


Table 9. Permitted effluent discharges in the New Jersey drainage basins studied, 1975--Continued

\begin{tabular}{|c|c|c|c|}
\hline \multicolumn{2}{|l|}{ Drainage basin } & \multirow{2}{*}{$\begin{array}{c}\text { NJPDES } \\
\text { permit } \\
\text { number }\end{array}$} & \multirow[b]{2}{*}{ Permitted effluent discharger } \\
\hline Monitoring-station name & $\begin{array}{l}\text { Monitoring- } \\
\text { station number }\end{array}$ & & \\
\hline \multirow[t]{40}{*}{ Passaic River at Two Bridges--Continued } & 01382000-Cont. & 20427 & CALDWELL, BOROUGH OF \\
\hline & & 21083 & VETERANS ADMINISTRATION \\
\hline & & 21181 & CEDAR HILL SCHOOL STP \\
\hline & & 21636 & NEW PROVIDENCE BOROUGH OF WTP \\
\hline & & 21938 & US ARMY NIKE 79/80 E HANOVER \\
\hline & & 22349 & ROCKAWAY VALLEY REG S.A. \\
\hline & & 22489 & WARREN TWP. SEWAGE AUTHORITY \\
\hline & & 22497 & STAGE IV SEWAGE TREATMENT PLT \\
\hline & & 22802 & WHITE MEADOW LAKE PROP.OWNERS \\
\hline & & 22845 & HARRISON BROOK \\
\hline & & 24431 & MONTVILLE TOWNSHIP MUA \\
\hline & & 24457 & OUR LADY OF THE MAGNIFICAT \\
\hline & & 24465 & PASSAIC TOWNSHIP OF \\
\hline & & 24511 & LIVINGSTON TOWNSHIP OF \\
\hline & & 24902 & HANOVER SEWERAGE AUTHORITY \\
\hline & & 24911 & BUTTERWORTH STP \\
\hline & & 24929 & WOODLAND STP \\
\hline & & 24937 & MADISON-CHATHAM JOINT MEETING \\
\hline & & 24970 & PARSIPPANY, TOWNSHIP OF \\
\hline & & 25496 & MORRISTOWN TOWN OF \\
\hline & & 25518 & FLORHAM PARK STP \\
\hline & & 25674 & ACTION TECHNOLOGY COMPANY \\
\hline & & 25712 & VIBRATION MOUNTING \& CONTROLS \\
\hline & & 25721 & BUTLER WATER DEPARTMENT \\
\hline & & 25739 & LITTON INDUSTRIES INC AIRTRON \\
\hline & & 26115 & -- \\
\hline & & 26603 & RANDOLPH HIGH SCHOOL STP \\
\hline & & 26654 & SISTERS OF CHARITY-ST. ELIZABE \\
\hline & & 26689 & ENGR. DEPT.-GREYSTONE PARK \\
\hline & & 26751 & ST MARY'S ABBEY \\
\hline & & 26867 & JEFFERSON TWP.-ROCK \\
\hline & & 27847 & -- \\
\hline & & 27961 & WATER POLLUTION CONTROL \\
\hline & & 28096 & PCI INC. \\
\hline & & 28339 & CAMPBELL-PRATT OIL CO \\
\hline & & 29394 & -- \\
\hline & & 29734 & FABRICATED PLASTICS \\
\hline & & 29912 & NEW JERSEY DEPT. OF TRANSPORTATION \\
\hline & & 29955 & RESISTOFLEX CORPORATION \\
\hline & & 29963 & GLASFLEX CORPORATION \\
\hline
\end{tabular}


Table 9. Permitted effluent discharges in the New Jersey drainage basins studied, 1975--Continued

\begin{tabular}{|c|c|c|c|}
\hline \multicolumn{2}{|l|}{ Drainage basin } & \multirow{2}{*}{$\begin{array}{c}\text { NJPDES } \\
\text { permit } \\
\text { number }\end{array}$} & \multirow[b]{2}{*}{ Permitted effluent discharger } \\
\hline Monitoring-station name & $\begin{array}{l}\text { Monitoring- } \\
\text { station number }\end{array}$ & & \\
\hline \multirow[t]{15}{*}{ Passaic River at Two Bridges--Continued } & 01382000-Cont. & 30287 & MONTVILLE TOWNSHIP MUA \\
\hline & & 30317 & MONTVILLE TOWNSHIP MUA \\
\hline & & 30911 & SCERBO BROTHERS INC \\
\hline & & 32166 & -- \\
\hline & & 32221 & LESLIE CO \\
\hline & & 32573 & NATIONAL MANUFACTURING CO \\
\hline & & 32808 & ROCKAWAY TOWNSQUARE MALL \\
\hline & & 33685 & CHAMPION INTL DAIRYPAK DIV \\
\hline & & 34053 & CARSAU CORPORATION \\
\hline & & 34134 & GREEN HAMMER METAL PRODUCTS CO \\
\hline & & 35050 & W P REALTY CO \\
\hline & & 35424 & BROE WN BOVERT-RECOMA INC \\
\hline & & 35777 & EXXON CENTRAL SERVICES \\
\hline & & 36081 & -- \\
\hline & & 52396 & PNEU HYDRO PRODUCTS INC \\
\hline \multirow[t]{2}{*}{ Wanaque River at Wanaque } & 01387000 & 30261 & WANAQUE BOROUGH SEWERAGE AUTHO \\
\hline & & 32395 & RINGWOOD PLAZA S T P \\
\hline \multirow[t]{8}{*}{ Saddle River at Lodi } & 01391500 & 1244 & DART INDUSTRIES INC \\
\hline & & 3221 & ALL PURPOSE ROLL LEAF \\
\hline & & 23931 & HEARTHSTONE AT MAHWAH \\
\hline & & 24791 & RIDGEWOOD VILLAGE COMPOST-FRAN \\
\hline & & 24813 & NORTHWEST BERGEN COUNTY S.A. \\
\hline & & 27910 & HOME FUEL OIL COMPANY \\
\hline & & 29726 & C M \& SON TRUCKING INC \\
\hline & & 33987 & IBM CORPORATION \\
\hline \multirow[t]{6}{*}{ SB Raritan River at Middle Valley } & 01396280 & 1236 & WELSH FARMS INC \\
\hline & & 21954 & MT OLIVE TOWNSHIP \\
\hline & & 22683 & SKYVIEW STP \\
\hline & & 23493 & WASHINGTON TWP M.U.A. \\
\hline & & 28304 & QUALITY INN \\
\hline & & 34975 & ADVANCED ENVIRON TECH CORPORATION \\
\hline \multirow[t]{6}{*}{ SB Raritan River at High Bridge } & 01396535 & 1236 & WELSH FARMS INC \\
\hline & & 21954 & MT OLIVE TOWNSHIP \\
\hline & & 22683 & SKYVIEW STP \\
\hline & & 23493 & WASHINGTON TWP M.U.A. \\
\hline & & 28304 & QUALITY INN \\
\hline & & 34975 & ADVANCED ENVIRON TECH CORPORATION \\
\hline Mulhockaway Creek at Van Syckel & 01396660 & 24091 & UNION TOWNSHIP BD OF ED \\
\hline
\end{tabular}


Table 9. Permitted effluent discharges in the New Jersey drainage basins studied, 1975--Continued

\begin{tabular}{|c|c|c|c|}
\hline \multicolumn{2}{|c|}{ Drainage basin } & \multirow{2}{*}{$\begin{array}{c}\text { NJPDES } \\
\text { permit } \\
\text { number }\end{array}$} & \multirow[b]{2}{*}{ Permitted effluent discharger } \\
\hline Monitoring-station name & $\begin{array}{l}\text { Monitoring- } \\
\text { station number }\end{array}$ & & \\
\hline \multirow[t]{16}{*}{ SB Raritan River at Three Bridges } & 01397400 & 1236 & WELSH FARMS INC \\
\hline & & 20389 & CLINTON SEWERAGE AUTHORITY \\
\hline & & 21954 & MT OLIVE TOWNSHIP \\
\hline & & 22047 & RARITAN TOWNSHIP STP \\
\hline & & 22144 & GLEN GARDNER CTR. FOR GERIATRI \\
\hline & & 22683 & SKYVIEW STP \\
\hline & & 23175 & CLINTON TOWNSHIP BD OF ED \\
\hline & & 23493 & WASHINGTON TWP M.U.A. \\
\hline & & 24091 & UNION TOWNSHIP BD OF ED \\
\hline & & 24571 & BORO OF CARTERET STP \\
\hline & & 26450 & LENTINE AGGREGATES \\
\hline & & 28304 & QUALITY INN \\
\hline & & 28436 & FLEMINGTON BOROUGH COUNCIL \\
\hline & & 28487 & YOUTH CORRECTIONAL INSTITUTION \\
\hline & & 28754 & MEENAN OIL CO INC \\
\hline & & 34975 & ADVANCED ENVIRON TECH CORPORATION \\
\hline NB Raritan River near Chester & 01398260 & 21334 & MENDHAM BOROUGH STP \\
\hline \multirow[t]{9}{*}{ NB Raritan River at Burnt Mills } & 01399120 & 21334 & MENDHAM BOROUGH STP \\
\hline & & 21881 & PEAPACK \& GLADSTONE, BOROUGH \\
\hline & & 26387 & BERNARDSVILLE, BOROUGH OF \\
\hline & & 26824 & CHESTER SHOPPING CENTER \\
\hline & & 27227 & JOHN Z. DELOREAN \\
\hline & & 28495 & BEDMINISTER STP \\
\hline & & 29637 & BERNARDSVILLE QUARRY, INC. \\
\hline & & 29807 & NJ DEPT. OF TRANS.-MAINTENANCE FAC \\
\hline & & 33995 & ENVIRONMENTAL DISPOSAL CORPORATION \\
\hline \multirow[t]{3}{*}{ Rockaway Creek at Whitehouse } & 01399700 & 2917 & OLDWICK MATERIALS INC \\
\hline & & 28452 & BEST A. M. CO INC \\
\hline & & 98922 & READINGTON-LEBANON SEWERAGE AU \\
\hline \multirow[t]{10}{*}{ Lamington River at Burnt Mills } & 01399780 & 876 & HERCULES CORPORATION \\
\hline & & 2861 & COUNTY CONCRETE CORPORATION \\
\hline & & 2917 & OLDWICK MATERIALS INC \\
\hline & & 20338 & BRANCHBURG, TOWNSHIP OF \\
\hline & & 21865 & LAMINGTON RIVER FARMS \\
\hline & & 22675 & ROXBURY-AJAX TER.STP, TOWNSHIP \\
\hline & & 22781 & VALLEY ROAD SEWERAGE CO \\
\hline & & 28452 & BEST A. M. CO INC \\
\hline & & 31755 & JIM SALERNO PONTIAC INC \\
\hline & & 98922 & READINGTON-LEBANON SEWERAGE AU \\
\hline
\end{tabular}


Table 9. Permitted effluent discharges in the New Jersey drainage basins studied, 1975--Continued

\begin{tabular}{|c|c|c|c|}
\hline \multicolumn{2}{|c|}{ Drainage basin } & \multirow{2}{*}{$\begin{array}{c}\text { NJPDES } \\
\text { permit } \\
\text { number }\end{array}$} & \multirow[b]{2}{*}{ Permitted effluent discharger } \\
\hline Monitoring-station name & $\begin{array}{l}\text { Monitoring- } \\
\text { station number }\end{array}$ & & \\
\hline \multirow[t]{40}{*}{ Raritan River at Manville } & 01400500 & 876 & -- \\
\hline & & 892 & EXXON CO USA \\
\hline & & 1139 & ETHICON INC \\
\hline & & 1236 & WELSH FARMS INC \\
\hline & & 1333 & NATIONAL STARCH \& CHEMICAL CO \\
\hline & & 1961 & DEVRO INC \\
\hline & & 2569 & RCA CORPORATION SOLID STATE PLANT \\
\hline & & 2861 & COUNTY CONCRETE CORPORATION \\
\hline & & 2917 & OLDWICK MATERIALS INC \\
\hline & & 3051 & WILSON-FIBERFIL/DART \& KRAFT \\
\hline & & 3158 & PRINTING PRODUCTS DIVISION \\
\hline & & 3298 & ETHYL CORPORATION \\
\hline & & 3638 & TAYLOR FORGE STAINLESS \\
\hline & & 3905 & 3 BRIDGES FARM \\
\hline & & 20338 & BRANCHBURG, TOWNSHIP OF \\
\hline & & 20354 & BRANCHBURG NESHANIC STP \\
\hline & & 20362 & BRANCHBURG, TOWNSHIP OF \\
\hline & & 20389 & CLINTON SEWERAGE AUTHORITY \\
\hline & & 20991 & SAINT BERNARDS CHURCH \\
\hline & & 21334 & MENDHAM BOROUGH STP \\
\hline & & 21865 & LAMINGTON RIVER FARMS \\
\hline & & 21881 & PEAPACK \& GLADSTONE BOROUGH \\
\hline & & 21954 & MT OLIVE TOWNSHIP \\
\hline & & 22047 & RARITAN TOWNSHIP STP \\
\hline & & 22144 & GLEN GARDNER CTR. FOR GERIATRI \\
\hline & & 22675 & -- \\
\hline & & 22683 & SKYVIEW STP \\
\hline & & 22781 & VALLEY ROAD SEWERAGE CO \\
\hline & & 23175 & CLINTON TOWNSHIP BD OF ED \\
\hline & & 23493 & WASHINGTON TWP M.U.A. \\
\hline & & 23914 & NJ TRANSIT RAIL OPERATIONS INC \\
\hline & & 24091 & UNION TOWNSHIP BD OF ED \\
\hline & & 24571 & -- \\
\hline & & 26387 & BERNARDSVILLE BOROUGH OF \\
\hline & & 26450 & LENTINE AGGREGATES \\
\hline & & 26697 & READINGTON TOWNSHIP BD OF ED \\
\hline & & 26824 & CHESTER SHOPPING CENTER \\
\hline & & 27227 & JOHN Z. DELOREAN \\
\hline & & 28304 & QUALITY INN \\
\hline & & 28436 & FLEMINGTON BOROUGH COUNCIL \\
\hline
\end{tabular}


Table 9. Permitted effluent discharges in the New Jersey drainage basins studied, 1975--Continued

\begin{tabular}{|c|c|c|c|}
\hline \multicolumn{2}{|c|}{ Drainage basin } & \multirow{2}{*}{$\begin{array}{c}\text { NJPDES } \\
\text { permit } \\
\text { number }\end{array}$} & \multirow[b]{2}{*}{ Permitted effluent discharger } \\
\hline Monitoring-station name & $\begin{array}{l}\text { Monitoring- } \\
\text { station number }\end{array}$ & & \\
\hline \multirow[t]{14}{*}{ Raritan River at Manville--Continued } & 01400500-Cont. & 28452 & BEST A. M. CO INC \\
\hline & & 28487 & YOUTH CORRECTIONAL INSTITUTION \\
\hline & & 28495 & BEDMINISTER STP \\
\hline & & 28754 & MEENAN OIL CO INC \\
\hline & & 29271 & TAYLOR OIL CO. \\
\hline & & 29637 & BERNARDSVILLE QUARRY INC. \\
\hline & & 29807 & NJ DEPT. OF TRANS.-MAINTENANCE FAC \\
\hline & & 29921 & CRESTLINE DIV OF N A PRODUCTS \\
\hline & & 31755 & JIM SALERNO PONTIAC INC \\
\hline & & 32328 & VIANINI PIPE INC \\
\hline & & 32662 & DARTCO MANUFACTURING \\
\hline & & 33995 & ENVIRONMENTAL DISPOSAL CORPORATION \\
\hline & & 34975 & ADVANCED ENVIRON TECH CORPORATION \\
\hline & & 98922 & READINGTON-LEBANON SEWERAGE AU \\
\hline \multirow[t]{4}{*}{ Millstone River at Grovers Mill } & 01400650 & 2666 & CARTER-WALLACE INC \\
\hline & & 22918 & ROOSEVELT, BOROUGH OF \\
\hline & & 23787 & EAST WINDSOR WATER POLLUTION \\
\hline & & 29475 & HIGHTSTOWN STP \\
\hline \multirow[t]{21}{*}{ Millstone River at Kingston } & 01401440 & 191 & COLUMBIAN CHEMICALS COMPANY \\
\hline & & 272 & DAVID SARNOFF RESEARCH CENTER \\
\hline & & 426 & IBM CORPORATION-CARD MFG PLANT \\
\hline & & 795 & MOBIL TECHNICAL CENTER \\
\hline & & 981 & STONY BROOK PLT \\
\hline & & 2666 & CARTER-WALLACE INC \\
\hline & & 4561 & COCA-COLA FOODS \\
\hline & & 20729 & NEW JERSEY TURNPIKE AUTHORITY \\
\hline & & 20770 & PRINCETON SEWER OPER. COMM. \\
\hline & & 22110 & EDUCATIONAL TESTING SERVICE \\
\hline & & 22560 & PRINCETON FARMS WTP \\
\hline & & 22918 & - \\
\hline & & 23205 & PRINCETON THEOLOGICAL SEMINARY \\
\hline & & 23787 & EAST WINDSOR WATER POLLUTION \\
\hline & & 24104 & THE LINPRO COMPANY \\
\hline & & 27731 & FMC CORPORATION \\
\hline & & 29475 & HIGHTSTOWN STP \\
\hline & & 31445 & FIRMENICH INCORPORATED \\
\hline & & 31950 & HUB SERVALL RECORD MFG CORPORATION \\
\hline & & 32611 & NATIONAL METALIZING \\
\hline & & 35319 & PENNINGTON FARMS WTP \\
\hline
\end{tabular}


Table 9. Permitted effluent discharges in the New Jersey drainage basins studied, 1975--Continued

\begin{tabular}{|c|c|c|c|}
\hline \multicolumn{2}{|c|}{ Drainage basin } & \multirow{2}{*}{$\begin{array}{c}\text { NJPDES } \\
\text { permit } \\
\text { number }\end{array}$} & \multirow[b]{2}{*}{ Permitted effluent discharger } \\
\hline Monitoring-station name & $\begin{array}{l}\text { Monitoring- } \\
\text { station number }\end{array}$ & & \\
\hline \multirow[t]{2}{*}{ Beden Brook near Rocky Hill } & 01401600 & 22390 & NORTH PRINCETON DEV SLF \\
\hline & & 32417 & BEDENS BROOK CLUB \\
\hline \multirow[t]{36}{*}{ Millstone River at Weston } & 01402540 & 191 & COLUMBLAN CHEMICALS COMPANY \\
\hline & & 272 & DAVID SARNOFF RESEARCH CENTER \\
\hline & & 426 & IBM CORPORATION-CARD MFG PLANT \\
\hline & & 795 & MOBIL TECHNICAL CENTER \\
\hline & & 981 & STONY BROOK PLT \\
\hline & & 2666 & CARTER-WALLACE INC \\
\hline & & 3255 & 3M COMPANY \\
\hline & & 4561 & COCA-COLA FOODS \\
\hline & & 20656 & GENERAL SERVICE ADMINISTRATION \\
\hline & & 20729 & -- \\
\hline & & 20770 & PRINCETON SEWER OPER. COMM. \\
\hline & & 20796 & PRINCETON SEWER OPER. COMM. \\
\hline & & 22110 & EDUCATIONAL TESTING SERVICE \\
\hline & & 22390 & NORTH PRINCETON DEV SLF \\
\hline & & 22560 & PRINCETON FARMS WTP \\
\hline & & 22918 & -- \\
\hline & & 23124 & MONTGOMERY TOWNSHIP OF \\
\hline & & 23205 & PRINCETON THEOLOGICAL SEMINARY \\
\hline & & 23523 & OKONITE CO., THE \\
\hline & & 23663 & CARRIER CLINIC \\
\hline & & 23787 & EAST WINDSOR WATER POLLUTION \\
\hline & & 24104 & THE LINPRO COMPANY \\
\hline & & 26140 & JOHNSON \& JOHNSON BABY PRODS. \\
\hline & & 26891 & MONTGOMERY STP 1 \\
\hline & & 26905 & MONTGOMERY STP\#2 TOWNSHIP OF \\
\hline & & 26913 & SLEEPY HOLLOW STP \\
\hline & & 27731 & FMC CORPORATION \\
\hline & & 29475 & HIGHTSTOWN STP \\
\hline & & 31119 & STONY BROOK REGIONAL S.A. \\
\hline & & 31445 & FIRMENICH INCORPORATIONORATED \\
\hline & & 31950 & HUB SERVALL RECORD MFG CORPORATION \\
\hline & & 32417 & BEDENS BROOK CLUB \\
\hline & & 32565 & INGERSOLL-RAND RESEARCH INC \\
\hline & & 32611 & NATIONAL METALIZING \\
\hline & & 35190 & NORTH BRUNSWICK, TOWNSHIP OF \\
\hline & & 35319 & PENNINGTON FARMS WTP \\
\hline Shark River near Neptune City & 01407705 & 21148 & MONMOUTH SERVICE AREA STP \\
\hline
\end{tabular}


Table 9. Permitted effluent discharges in the New Jersey drainage basins studied, 1975--Continued

\begin{tabular}{|c|c|c|c|}
\hline \multicolumn{2}{|l|}{ Drainage basin } & \multirow{2}{*}{$\begin{array}{c}\text { NJPDES } \\
\text { permit } \\
\text { number }\end{array}$} & \multirow[b]{2}{*}{ Permitted effluent discharger } \\
\hline Monitoring-station name & $\begin{array}{c}\text { Monitoring- } \\
\text { station number }\end{array}$ & & \\
\hline Jumping Brook near Neptune City & 01407760 & 3573 & FINETEX INC \\
\hline \multirow[t]{2}{*}{ Marsh Bog Brook at Squankum } & 01407997 & 26638 & FARMINGDALE GARDEN APTS. \\
\hline & & 28622 & FREQUENCY ENGINEERING LABS \\
\hline \multirow[t]{3}{*}{ Toms River near Toms River } & 01408500 & 4120 & CIBA-GEIGY CORPORATION \\
\hline & & 35041 & MAPLE GLEN PARK \\
\hline & & 35653 & FOUNTAINHEAD PARK INC \\
\hline \multirow[t]{2}{*}{ Hammonton Creek at Westcoatville } & 01409416 & 24210 & WHITEHALL LABORATORIES \\
\hline & & 25160 & HAMMONTON WASTEWATER TREATMENT \\
\hline Great Egg Harbor near Sicklerville & 01410784 & 26972 & BERLIN BOROUGH WPC PLANT \\
\hline Great Egg Harbor near Blue Anchor & 01410820 & 26972 & BERLIN BOROUGH WPC PLANT \\
\hline \multirow[t]{4}{*}{ Great Egg Harbor at Weymouth } & 01411110 & 4588 & AKZO CHEMICALS INC. \\
\hline & & 26409 & LINDENWOLD BOROUGH MUA \\
\hline & & 26522 & CENTRAL MAINT MP 275 \\
\hline & & 26972 & BERLIN BOROUGH WPC PLANT \\
\hline \multirow[t]{6}{*}{ Maurice River at Norma } & 01411500 & 4103 & SHIELDALLOY CORPORATION \\
\hline & & 5312 & O I GLASS CONTAINER STS INC \\
\hline & & 20125 & MILES PETROLEUM INC \\
\hline & & 25658 & PIONEER METAL FINISHERS INC \\
\hline & & 32361 & RON-SON MUSHROOM PROD INC \\
\hline & & 36129 & MARSHALL SERVICE INC \\
\hline Cohansey River at Seeley & 01412800 & 24147 & CUMBERLAND COUNTY UTILITIES \\
\hline \multirow[t]{7}{*}{ Paulins Kill at Blairstown } & 01443500 & 4791 & LIMECREST PLANT SPARTA \\
\hline & & 20184 & NEWTON, TOWN OF \\
\hline & & 22063 & SUSSEX COUNTY BRD FREEHOLDERS \\
\hline & & 24163 & 'BIG N' SHOPPING CENTER STP \\
\hline & & 26701 & SUSSEX COUNTY BRD FREEHOLDERS \\
\hline & & 27057 & SPARTA TWP PLAZA SEWAGE PLANT \\
\hline & & 28819 & HART \& ILIFF FUEL OIL COMPANY \\
\hline \multirow[t]{3}{*}{ Musconetcong River at Outlet of Lake Hopatcong } & 01455500 & 21105 & ARTHUR STANLICK SCHOOL \\
\hline & & 21156 & CONSOLIDATED SCHOOL \\
\hline & & 26212 & MT ARLINGTON SANITATION CORPORATION \\
\hline \multirow[t]{5}{*}{ Musconetcong River at Beatyestown } & 01456200 & 4600 & US MINERAL PRODUCTS \\
\hline & & 5568 & COOKE COLOR \& CHEMICAL CO. \\
\hline & & 21105 & ARTHUR STANLICK SCHOOL \\
\hline & & 21156 & CONSOLIDATED SCHOOL \\
\hline & & 21369 & HACKETTSTOWN MUA \\
\hline
\end{tabular}


Table 9. Permitted effluent discharges in the New Jersey drainage basins studied, 1975--Continued

\begin{tabular}{|c|c|c|c|}
\hline \multicolumn{2}{|l|}{ Drainage basin } & \multirow{2}{*}{$\begin{array}{c}\text { NJPDES } \\
\text { permit } \\
\text { number }\end{array}$} & \multirow[b]{2}{*}{ Permitted effluent discharger } \\
\hline Monitoring-station name & $\begin{array}{l}\text { Monitoring- } \\
\text { station number }\end{array}$ & & \\
\hline \multirow[t]{2}{*}{ Musconetcong River at Beatyestown--Continued } & 01456200-Cont. & 22632 & BYRAM TOWNSHIP BD OF ED \\
\hline & & 26212 & MT ARLINGTON SANITATION CORPORATION \\
\hline \multirow[t]{12}{*}{ Musconetcong River at Riegelsville } & 01457400 & 4421 & RIEGEL PRODUCTS CORPORATION \\
\hline & & 4448 & WARREN GLEN MILL \\
\hline & & 4600 & US MINERAL PRODUCTS \\
\hline & & 5568 & -- \\
\hline & & 21105 & ARTHUR STANLICK SCHOOL \\
\hline & & 21156 & CONSOLIDATED SCHOOL \\
\hline & & 21369 & HACKETTSTOWN MUA \\
\hline & & 22632 & BYRAM TOWNSHIP BD OF ED \\
\hline & & 25569 & BLOOMSBURY WATER COMPANY \\
\hline & & 26212 & MT ARLINGTON SANITATION CORPORATION \\
\hline & & 28592 & DIAMOND HILL ESTATES SEWAGE CO \\
\hline & & 31208 & ASBURY GRAPHITE MILLS INC \\
\hline \multirow[t]{2}{*}{ Wickechcoke Creek at Stockton } & 01461300 & 27537 & MAGNESIUM ELEKTRON INC \\
\hline & & 27561 & DELAWARE TOWNSHIP MUA \\
\hline \multirow[t]{6}{*}{ Crosswicks Creek at Extonville } & 01464500 & 4855 & U.S. ARMY FT. DIX \& TRNG. CNTR \\
\hline & & 21091 & JEFFERSON MIDDLE \& HIGH SCHOOL \\
\hline & & 21407 & PLUMSTEAD TWP SCHOOL DIST. \\
\hline & & 22985 & WRIGHTSTOWN MUA \\
\hline & & 27464 & HANOVER MOBILE VILLAGE \\
\hline & & 27511 & CALIFORNIA VILLA MOBILE \\
\hline Doctors Creek at Allentown & 01464515 & 20206 & ALLENTOWN WTP \\
\hline \multirow[t]{3}{*}{ SB Rancocas Creek at Vincentown } & 01465850 & 21768 & NEW LISBON STATE SCHOOL \\
\hline & & 23736 & SOUTHAMPTON SEWERAGE CO \\
\hline & & 33367 & STOKES OF VINCENTOWN INC \\
\hline \multirow[t]{2}{*}{ NB Rancocas Creek at Brownsmills } & 01467000 & 21733 & PEMBERTON \\
\hline & & 28665 & MOBILE ESTATES OF SOUTHAMPTON \\
\hline NB Pennsauken Creek near Moorestown & 01467069 & 23981 & MOUNT LAUREL MUA \\
\hline \multirow[t]{2}{*}{ SB Pennsauken Creek at Cherry Hill } & 01467081 & 24040 & WOODSTREAM STP \\
\hline & & 31879 & MAPLE SHADE TOWNSHIP OF \\
\hline \multirow[t]{5}{*}{ Cooper River at Lawnside } & 01467140 & 3999 & HUSSMANN REFRIGERATOR CO \\
\hline & & 20621 & LAWNSIDE BOROUGH OF \\
\hline & & 21652 & SOMERDALE, BOROUGH OF \\
\hline & & 22403 & VOORHEES TOWNSHIP \\
\hline & & 25101 & CHERRY HILL TOWNSHIP \\
\hline
\end{tabular}


Table 9. Permitted effluent discharges in the New Jersey drainage basins studied, 1975--Continued

\begin{tabular}{|c|c|c|c|}
\hline \multicolumn{2}{|l|}{ Drainage basin } & \multirow{2}{*}{$\begin{array}{c}\text { NJPDES } \\
\text { permit } \\
\text { number }\end{array}$} & \multirow[b]{2}{*}{ Permitted effluent discharger } \\
\hline Monitoring-station name & $\begin{array}{l}\text { Monitoring- } \\
\text { station number }\end{array}$ & & \\
\hline \multirow{2}{*}{ Cooper River at Lawnside--Continued } & 01467140-Cont. & 25119 & CHERRY HILL TOWNSHIP \\
\hline & & 26361 & GIBBSBORO SEWAGE CORPORATION \\
\hline SB Big Timber Creek at Blackwood Terrace & 01467329 & 29840 & CAMDEN C'NTY SEWAGE TRETMT PLT \\
\hline Raccoon Creek near Swedesboro & 01477120 & 20532 & HARRISON TWP-MULLICA HILL STP \\
\hline
\end{tabular}




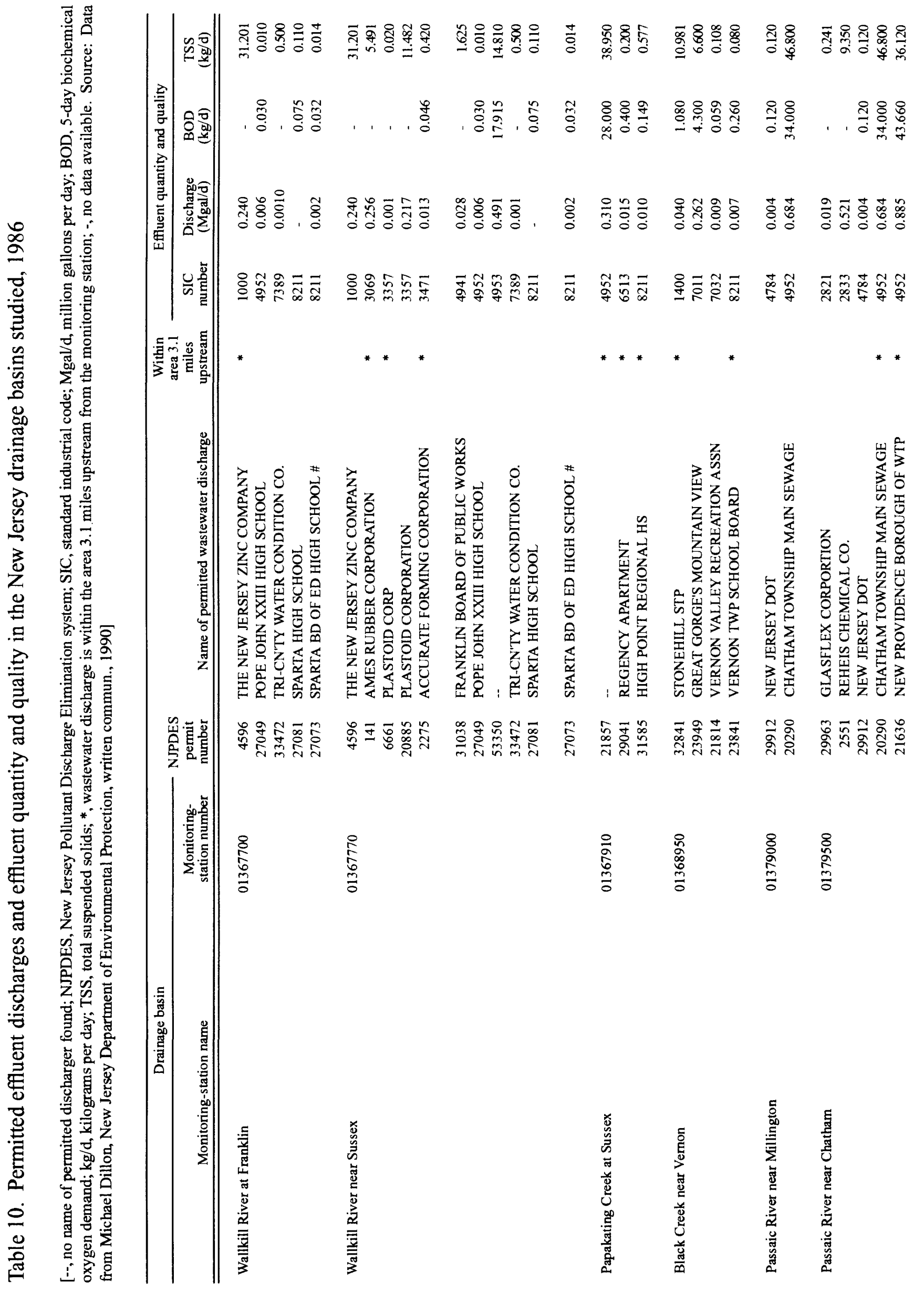




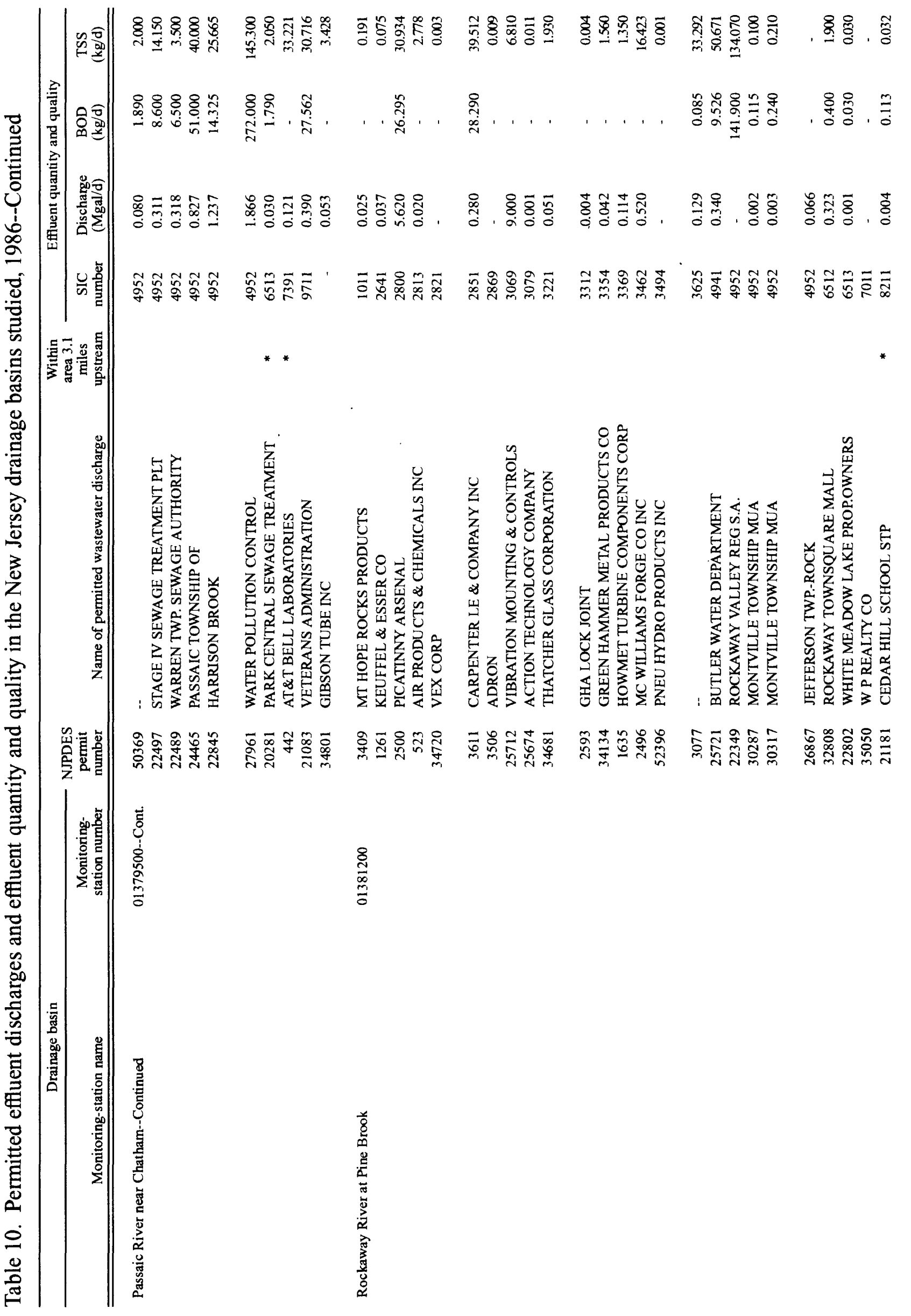




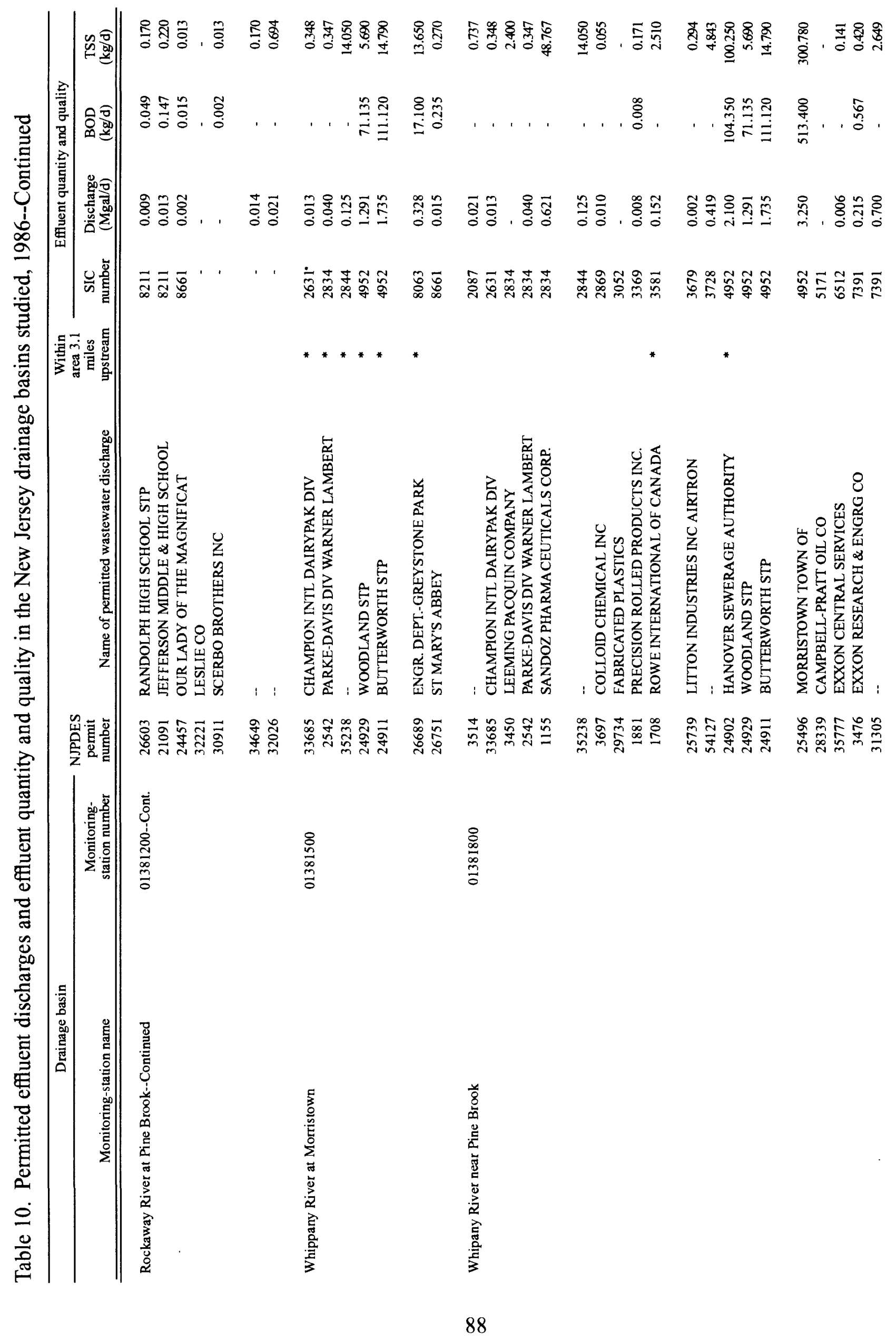




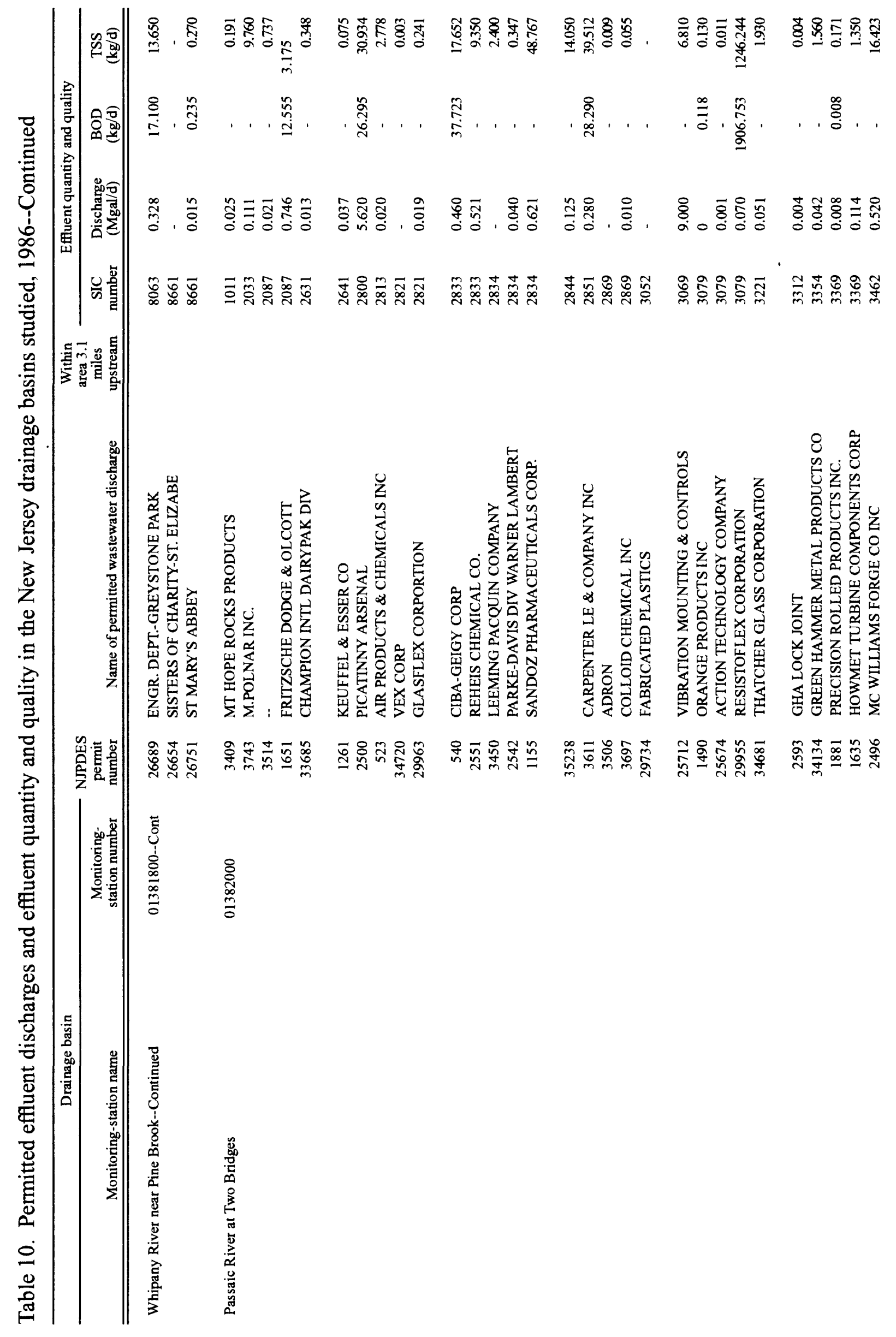




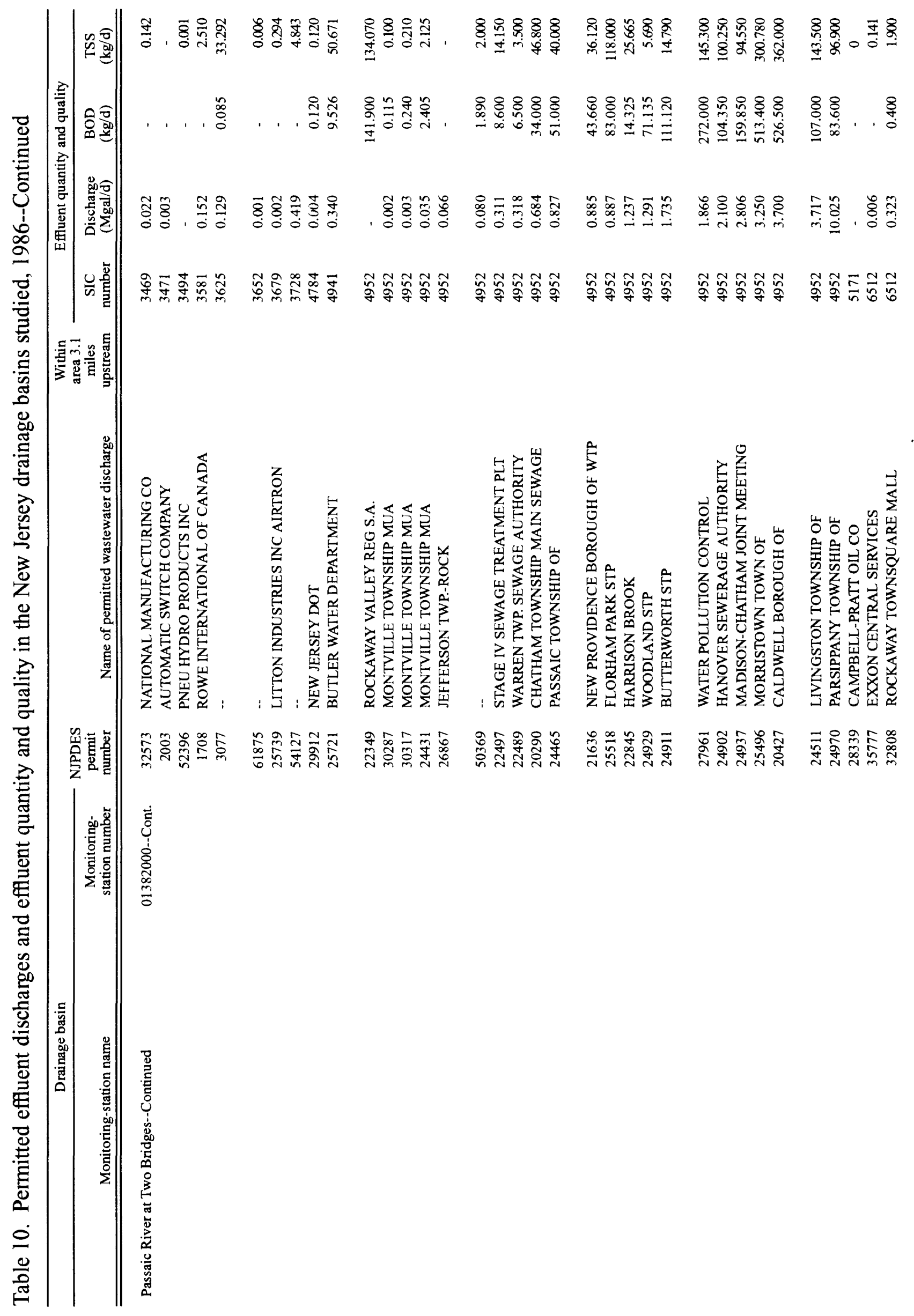




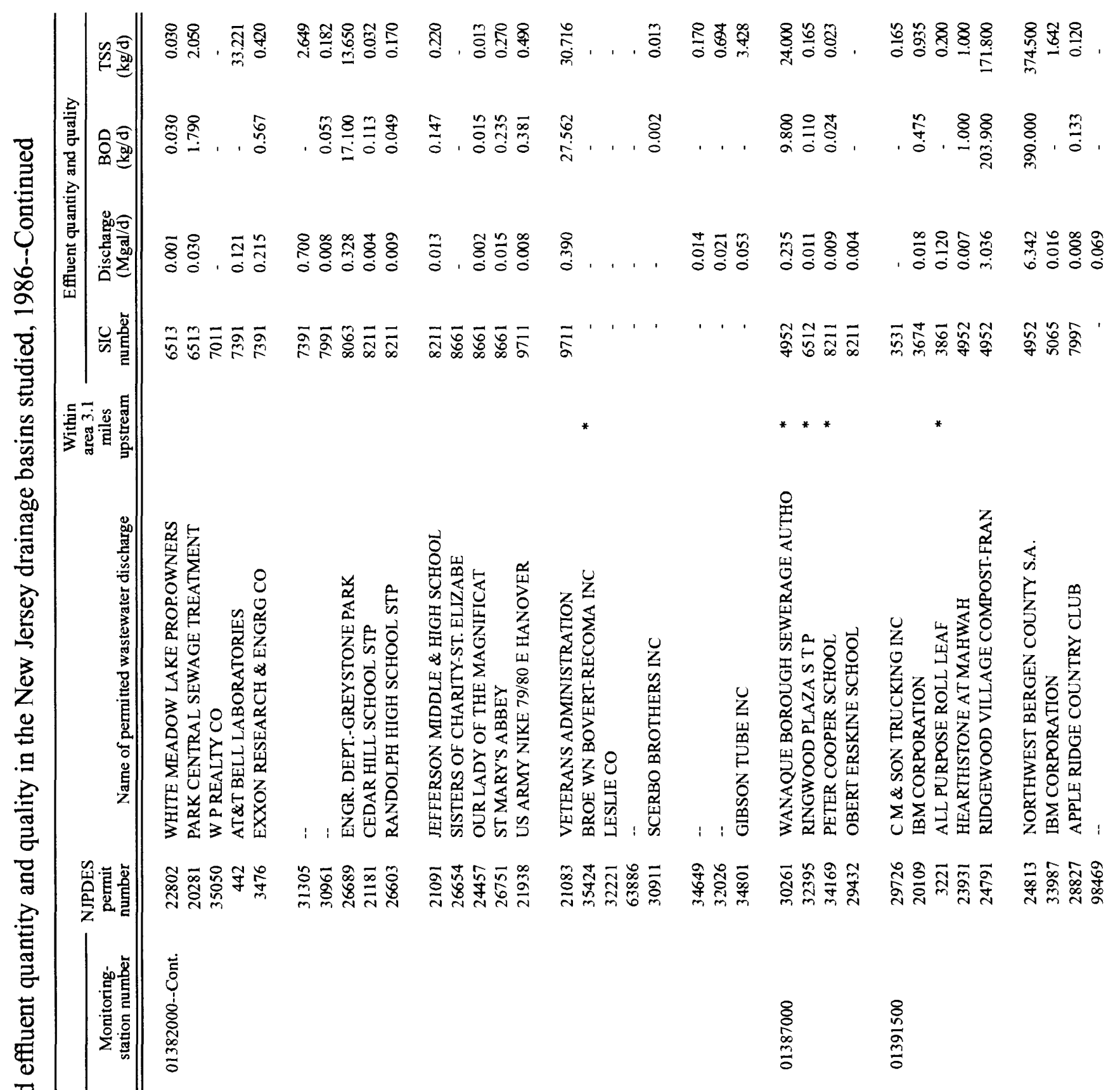




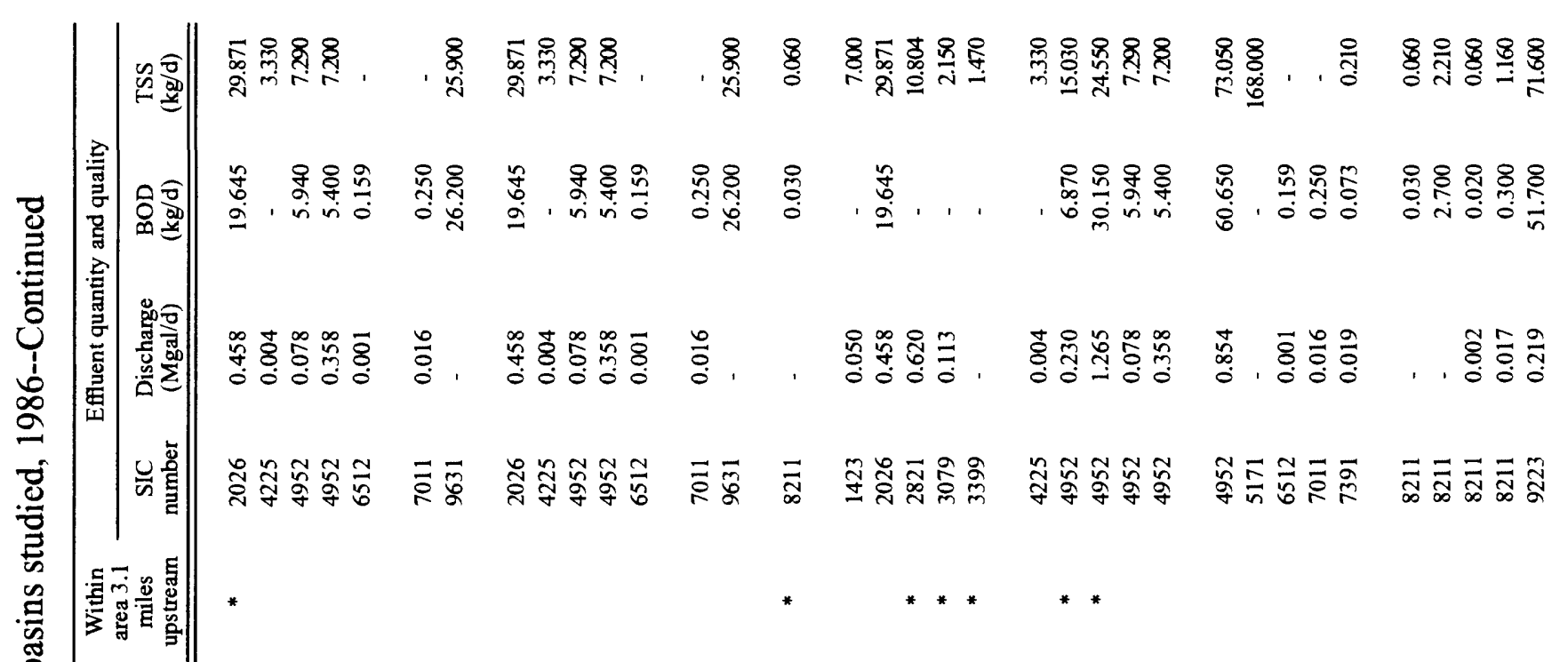




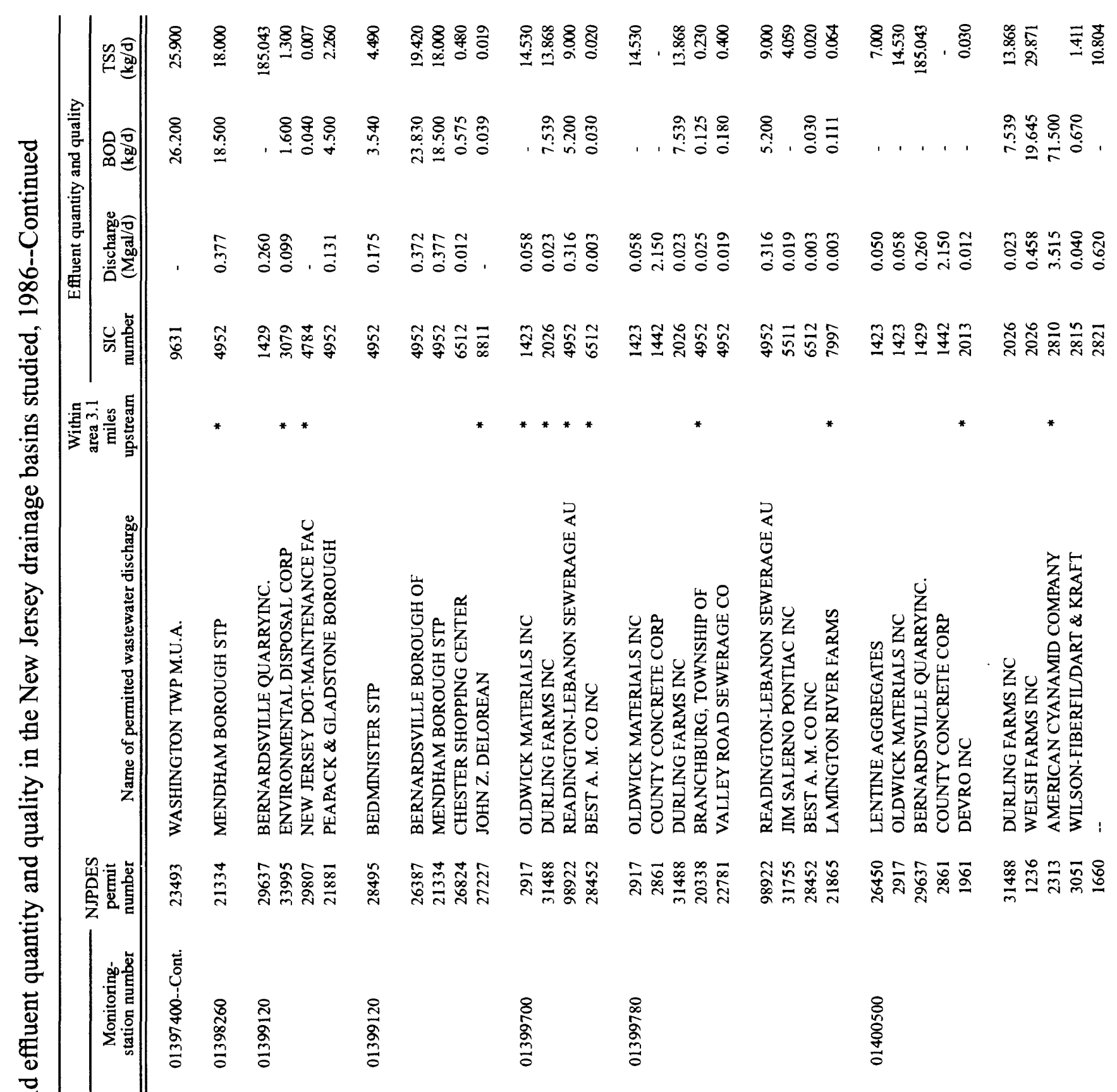




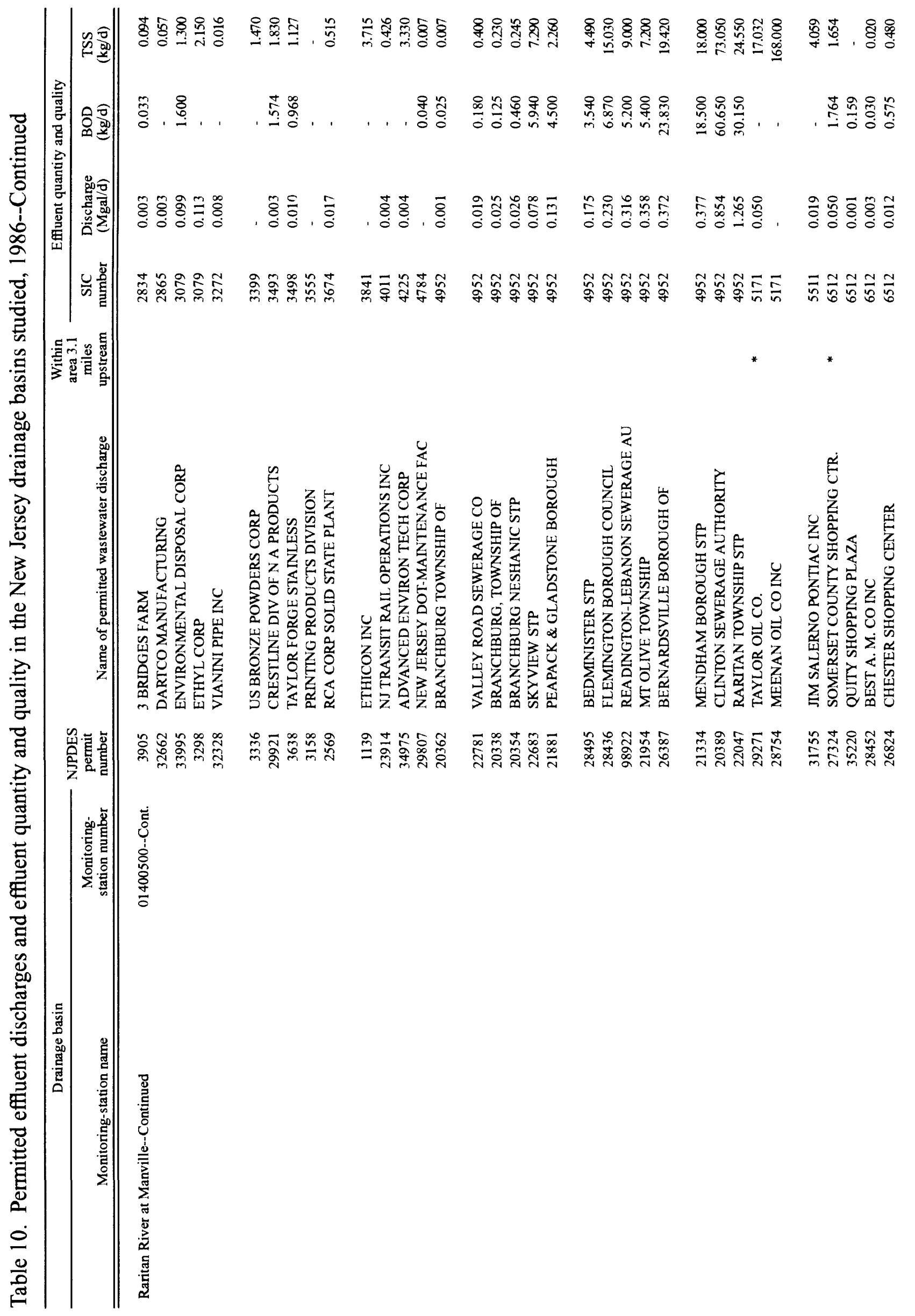




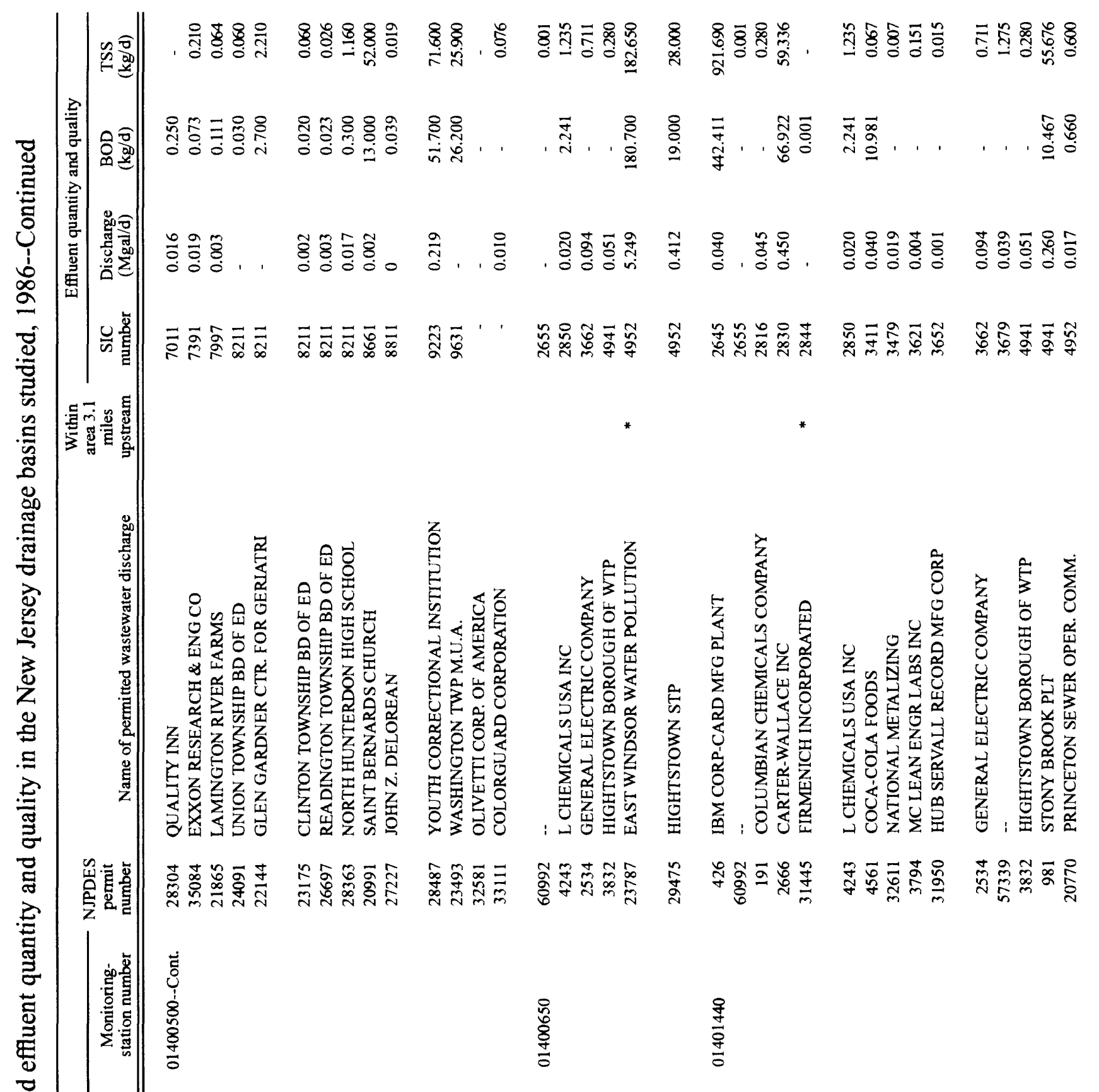




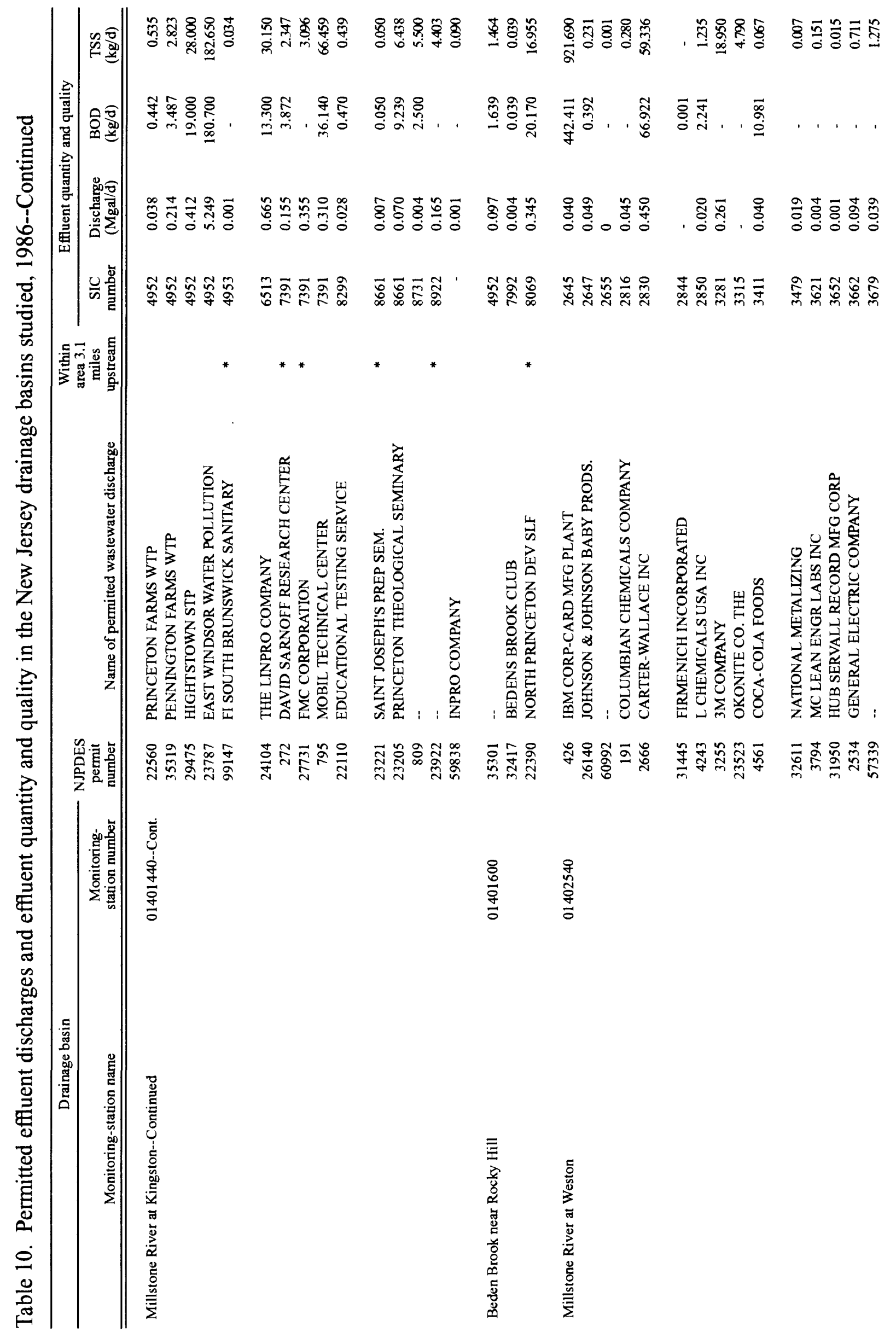




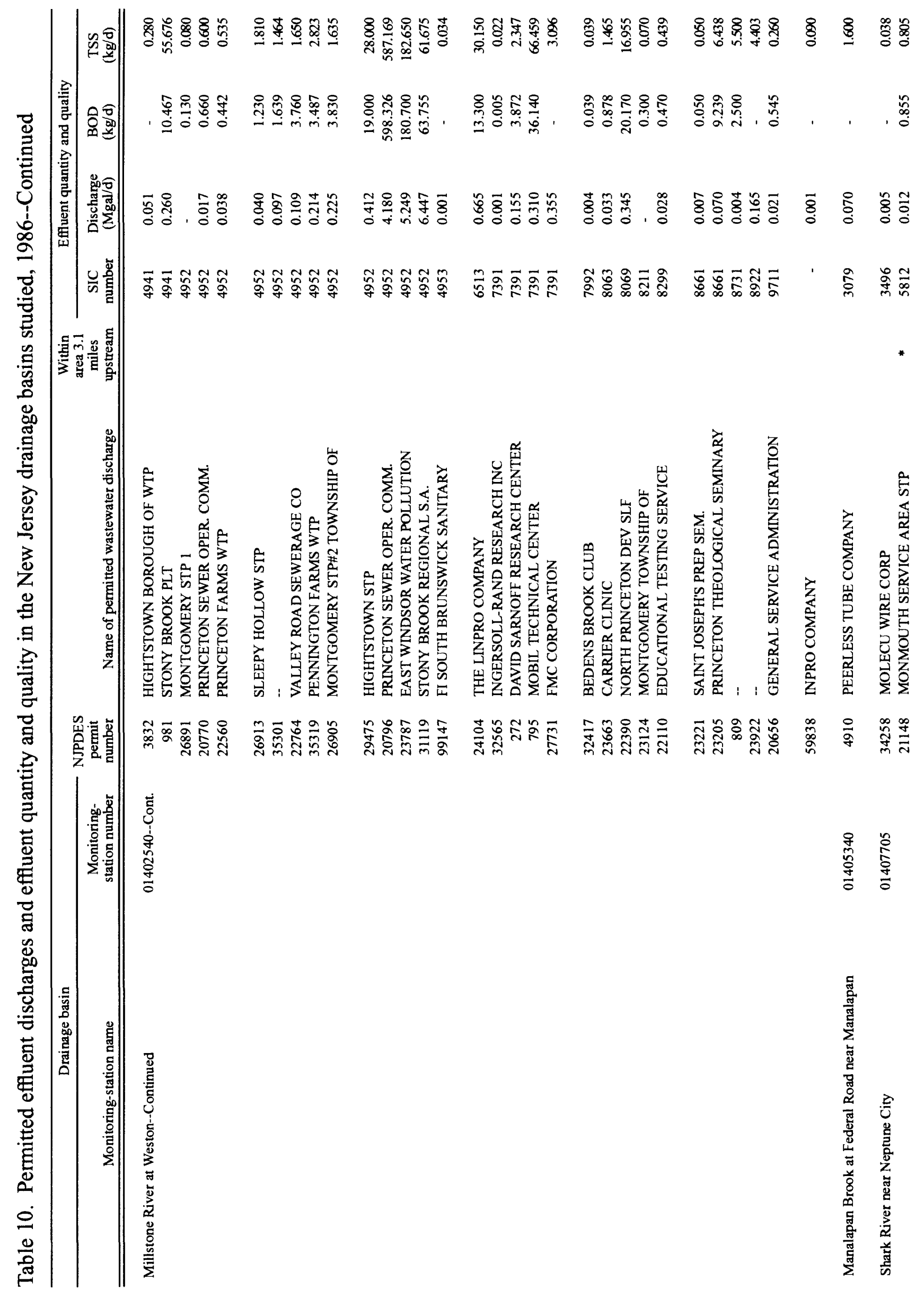




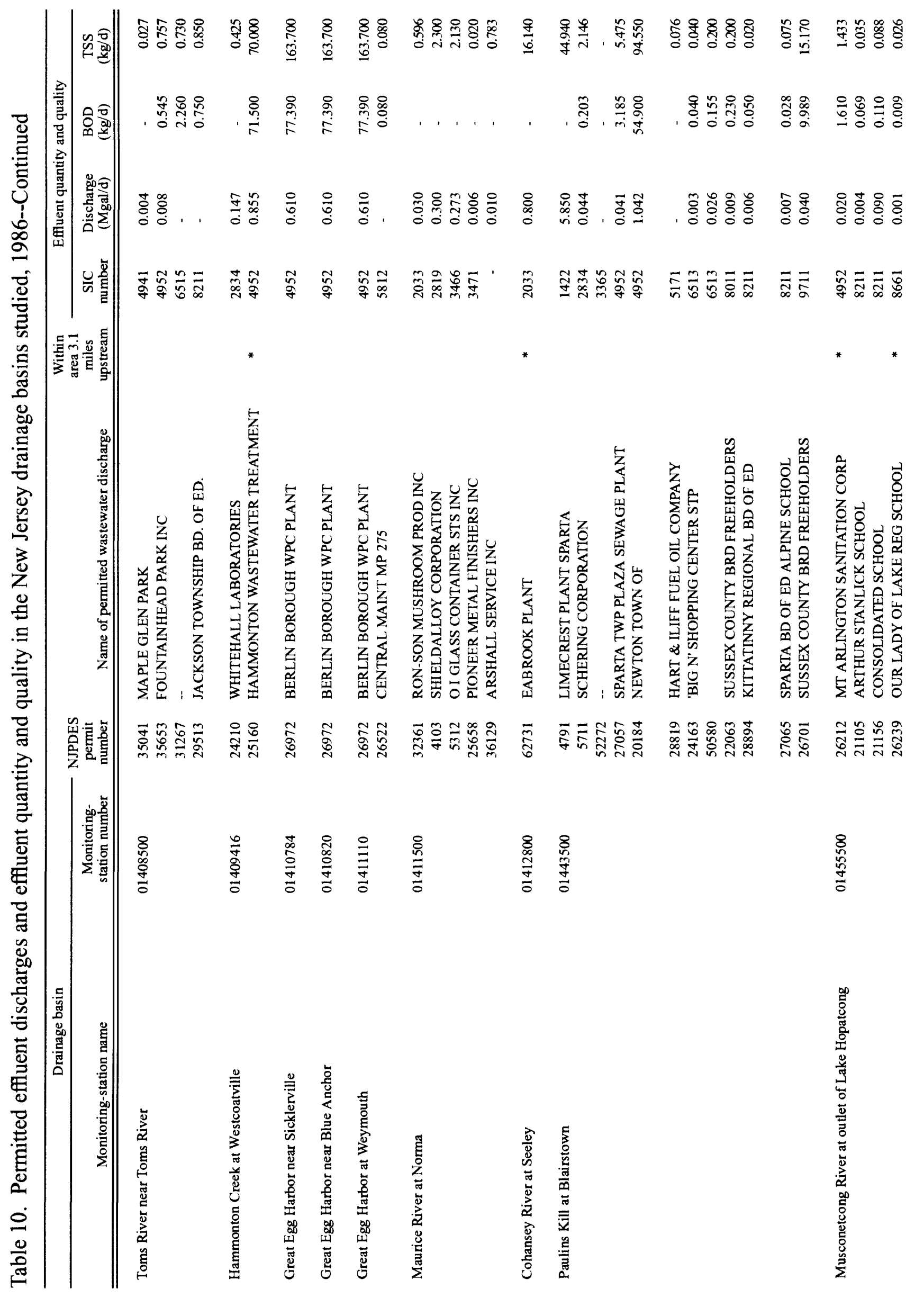




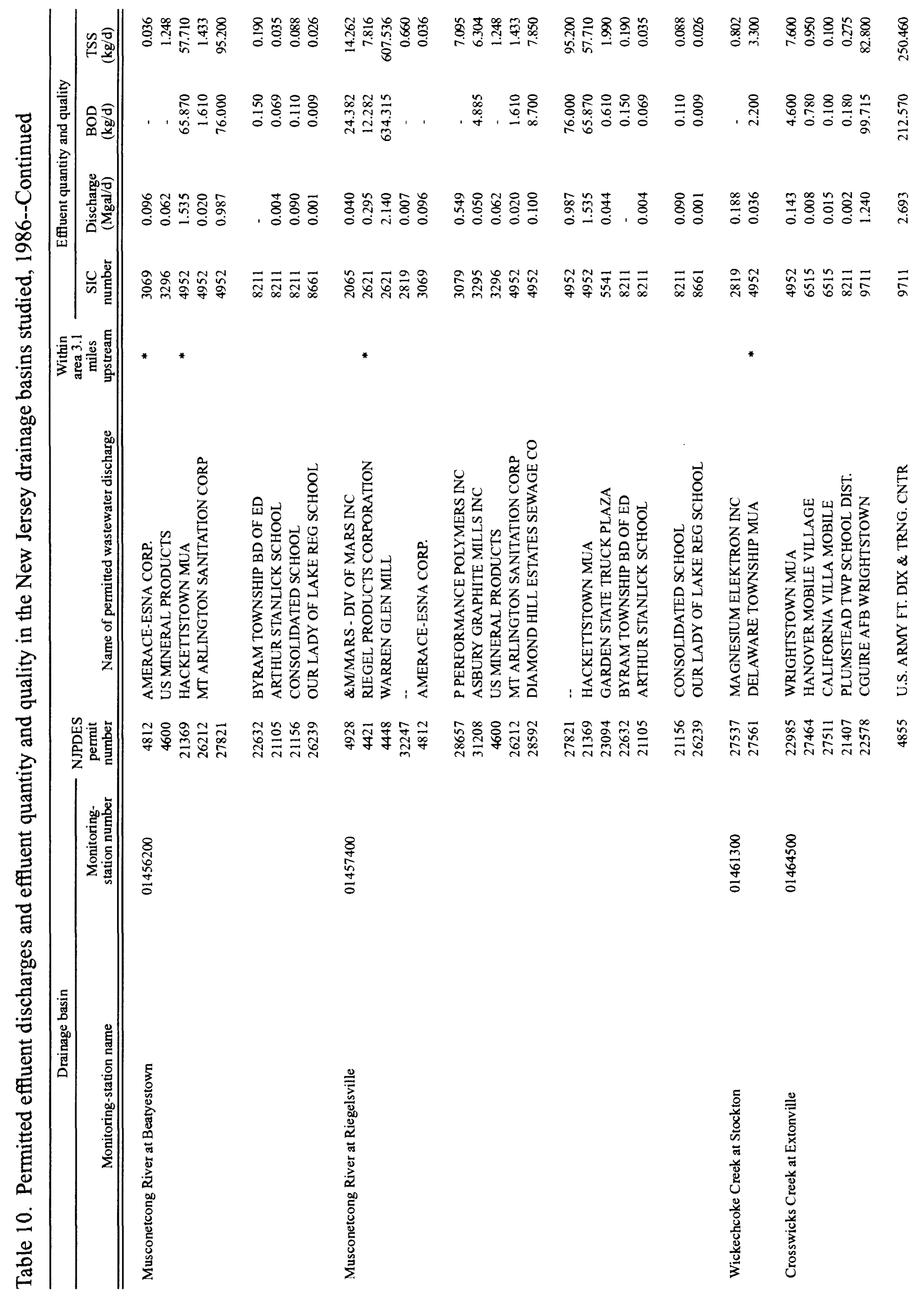




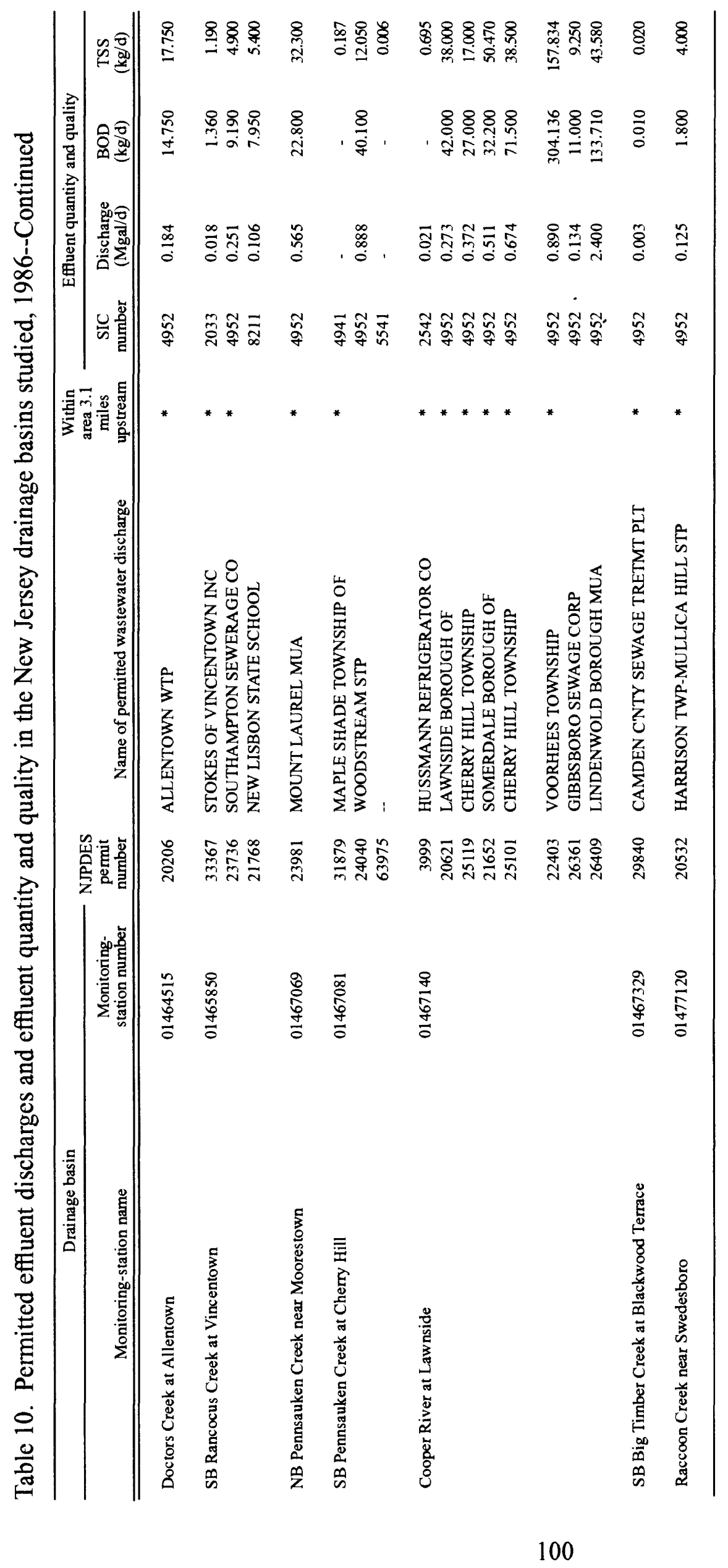


Table 13. Road-salt application in Regions I-IV in New Jersey by winter season, I975-76 through I986-87

[All data are tons applied. Source: A.T. Woodrow, New Jersey Department of Transportation, written commun., 1989]

\begin{tabular}{|c|c|c|c|c|}
\hline $\begin{array}{l}\text { Region }{ }^{1} \text { and } \\
\text { winter season }\end{array}$ & $\begin{array}{l}\text { Sodium } \\
\text { chloride }\end{array}$ & $\begin{array}{l}\text { Calcium chloride } \\
\text { in solution }\end{array}$ & $\begin{array}{l}\text { Calcium chloride } \\
\text { bulk (dry) }\end{array}$ & $\begin{array}{l}\text { Sodium chloride/ } \\
\text { calcium chloride } \\
\text { premix }\end{array}$ \\
\hline \multicolumn{5}{|l|}{ Region 1} \\
\hline $1975-76$ & 14,367 & 0 & 2,682 & 0 \\
\hline $1976-77$ & 4,722 & 0 & 1,026 & 9,106 \\
\hline $1977-78$ & 10,738 & 0 & 1,678 & 12,150 \\
\hline $1978-79$ & 8,129 & 0 & 1,123 & 12,199 \\
\hline $1979-80$ & 1,686 & 0 & 187 & 9,318 \\
\hline $1980-81$ & 5,008 & 0 & 555 & 11,416 \\
\hline $1981-82$ & 29,015 & 154,994 & 613 & 3,181 \\
\hline $1982-83$ & 18,365 & 115,193 & 62 & 0 \\
\hline $1983-84$ & 34,983 & 239,278 & 642 & 0 \\
\hline $1984-85$ & 29,161 & 188,226 & 188 & 0 \\
\hline $1985-86$ & 34,819 & 242,995 & 82 & 0 \\
\hline $1986-87$ & 38,753 & 237,680 & 2 & 0 \\
\hline \multicolumn{5}{|l|}{ Region 2} \\
\hline $1975-76$ & 8,278 & 0 & 1,570 & 0 \\
\hline $1976-77$ & 4,310 & 0 & 682 & 0 \\
\hline $1977-78$ & 7,149 & 5,553 & 1,170 & 5,553 \\
\hline $1978-79$ & 6,071 & 4,898 & 882 & 4,898 \\
\hline $1979-80$ & 1,889 & 4,370 & 345 & 4,370 \\
\hline $1980-81$ & 1,483 & 7,650 & 104 & 7,650 \\
\hline $1981-82$ & 11,705 & 61,990 & 234 & 3,655 \\
\hline $1982-83$ & 11,146 & 58,357 & 9 & 77 \\
\hline $1983-84$ & 18,983 & 131,506 & 0 & 0 \\
\hline $1984-85$ & 17,784 & 115,684 & 0 & 0 \\
\hline $1985-86$ & 19,807 & 114,050 & 0 & 0 \\
\hline $1986-87$ & 21,615 & 98,231 & 0 & 0 \\
\hline
\end{tabular}


Table 13. Road-salt application in Regions I-IV in New Jersey by winter season, 1975-76 through 1986-87--Continued

[All data are tons applied. Source: A.T. Woodrow, New Jersey Department of Transportation, written commun., 1989]

\begin{tabular}{|c|c|c|c|c|}
\hline $\begin{array}{l}\text { Region }{ }^{1} \text { and } \\
\text { winter season }\end{array}$ & $\begin{array}{l}\text { Sodium } \\
\text { chloride }\end{array}$ & $\begin{array}{l}\text { Calcium chloride } \\
\text { in solution }\end{array}$ & $\begin{array}{l}\text { Calcium chloride } \\
\text { bulk (dry) }\end{array}$ & $\begin{array}{l}\text { Sodium chloride/ } \\
\text { calcium chloride } \\
\text { premix }\end{array}$ \\
\hline \multicolumn{5}{|l|}{ Region 3} \\
\hline $1975-76$ & 11,589 & 0 & 820 & 0 \\
\hline $1976-77$ & 4,175 & 0 & 94 & 6,676 \\
\hline $1977-78$ & 4,118 & 0 & 200 & 9,144 \\
\hline $1978-79$ & 1,500 & 0 & 0 & 9,871 \\
\hline $1979-80$ & 1,302 & 0 & 0 & 5,220 \\
\hline $1980-81$ & 1,952 & 0 & 5 & 6,656 \\
\hline $1981-82$ & 9,968 & 34,837 & 77 & 4,796 \\
\hline $1982-83$ & 7,056 & 43,028 & 8 & 452 \\
\hline $1983-84$ & 14,327 & 99,797 & 0 & 0 \\
\hline $1984-85$ & 10,992 & 81,849 & 288 & 0 \\
\hline $1985-86$ & 13,153 & 90,243 & 0 & 0 \\
\hline $1986-87$ & 21,164 & 126,830 & 0 & 0 \\
\hline \multicolumn{5}{|l|}{ Region 4} \\
\hline $1975-76$ & 4,518 & 0 & 326 & 0 \\
\hline $1976-77$ & 1,811 & 0 & 93 & 4,102 \\
\hline $1977-78$ & 1,145 & 0 & 129 & 5,872 \\
\hline $1978-79$ & 839 & 0 & 81 & 6,600 \\
\hline $1979-80$ & 909 & 0 & 38 & 4,106 \\
\hline $1980-81$ & 1,286 & 0 & 3 & 2,971 \\
\hline $1981-82$ & 4,289 & 22,747 & 53 & 45 \\
\hline $1982-83$ & 3,800 & 26,472 & 514 & 0 \\
\hline $1983-84$ & 7,409 & 79,923 & 192 & 0 \\
\hline $1984-85$ & 9,147 & 82,417 & 0 & 0 \\
\hline $1985-86$ & 12,769 & 103,245 & 60 & 0 \\
\hline $1986-87$ & 15,867 & 128,040 & 0 & 0 \\
\hline
\end{tabular}

${ }^{1}$ Regions described on page 34 . 
Table I4. Estimated road-salt application in the New Jersey drainage basins studied, in terms of chloride, sodium, and calcium by basin and winter season, 1975-76 through 1986-87

[Source: A.T. Woodrow, New Jersey Department of Transportation, written commun., 1989]

\begin{tabular}{|c|c|c|c|c|c|}
\hline \multicolumn{2}{|c|}{ Drainage basin } & \multirow[b]{2}{*}{$\begin{array}{l}\text { Winter } \\
\text { season }\end{array}$} & \multicolumn{3}{|c|}{ Application rate, in tons per square mile } \\
\hline Monitoring-station name & $\begin{array}{l}\text { Monitoring- } \\
\text { station number }\end{array}$ & & Chloride & Sodium & Calcium \\
\hline \multirow[t]{3}{*}{ Wallkill River at Franklin } & 01367700 & $\begin{array}{l}1975-76 \\
1976-77 \\
1977-78 \\
1978-79 \\
1979-80\end{array}$ & $\begin{array}{l}4.93 \\
4.29 \\
7.10 \\
6.20 \\
3.23\end{array}$ & $\begin{array}{l}2.64 \\
2.26 \\
3.84 \\
3.36 \\
1.74\end{array}$ & $\begin{array}{l}0.68 \\
0.65 \\
0.94 \\
0.80 \\
0.44\end{array}$ \\
\hline & & $\begin{array}{l}1980-81 \\
1981-82 \\
1982-83 \\
1983-84 \\
1984-85\end{array}$ & $\begin{array}{r}4.91 \\
9.53 \\
5.36 \\
10.38 \\
8.54\end{array}$ & $\begin{array}{l}2.67 \\
5.82 \\
3.38 \\
6.43 \\
5.36\end{array}$ & $\begin{array}{l}0.62 \\
0.36 \\
0.07 \\
0.27 \\
0.13\end{array}$ \\
\hline & & $\begin{array}{l}1985-86 \\
1986-87\end{array}$ & $\begin{array}{l}10.16 \\
11.27\end{array}$ & $\begin{array}{l}6.40 \\
7.13\end{array}$ & $\begin{array}{l}0.13 \\
0.11\end{array}$ \\
\hline \multirow[t]{3}{*}{ Wallkill River near Sussex } & 01367770 & $\begin{array}{l}1975-76 \\
1976-77 \\
1977-78 \\
1978-79 \\
1979-80\end{array}$ & $\begin{array}{l}4.93 \\
4.29 \\
7.10 \\
6.20 \\
3.23\end{array}$ & $\begin{array}{l}2.64 \\
2.26 \\
3.84 \\
3.36 \\
1.74\end{array}$ & $\begin{array}{l}0.68 \\
0.65 \\
0.94 \\
0.80 \\
0.44\end{array}$ \\
\hline & & $\begin{array}{l}1980-81 \\
1981-82 \\
1982-83 \\
1983-84 \\
1984-85\end{array}$ & $\begin{array}{r}4.91 \\
9.53 \\
5.36 \\
10.38 \\
8.54\end{array}$ & $\begin{array}{l}2.67 \\
5.82 \\
3.38 \\
6.43 \\
5.36\end{array}$ & $\begin{array}{l}0.62 \\
0.36 \\
0.07 \\
0.27 \\
0.13\end{array}$ \\
\hline & & $\begin{array}{l}1985-86 \\
1986-87\end{array}$ & $\begin{array}{l}10.16 \\
11.27\end{array}$ & $\begin{array}{l}6.40 \\
7.13\end{array}$ & $\begin{array}{l}0.13 \\
0.11\end{array}$ \\
\hline \multirow[t]{3}{*}{ Papakating Creek at Sussex } & 01367910 & $\begin{array}{l}1975-76 \\
1976-77 \\
1977-78 \\
1978-79 \\
1979-80\end{array}$ & $\begin{array}{l}4.93 \\
4.29 \\
7.10 \\
6.20 \\
3.23\end{array}$ & $\begin{array}{l}2.64 \\
2.26 \\
3.84 \\
3.36 \\
1.74\end{array}$ & $\begin{array}{l}0.68 \\
0.65 \\
0.94 \\
0.80 \\
0.44\end{array}$ \\
\hline & & $\begin{array}{l}1980-81 \\
1981-82 \\
1982-83 \\
1983-84 \\
1984-85\end{array}$ & $\begin{array}{r}4.91 \\
9.53 \\
5.36 \\
10.38 \\
8.54\end{array}$ & $\begin{array}{l}2.67 \\
5.82 \\
3.38 \\
6.43 \\
5.36\end{array}$ & $\begin{array}{l}0.62 \\
0.36 \\
0.07 \\
0.27 \\
0.13\end{array}$ \\
\hline & & $\begin{array}{r}1985-86 \\
1986-87\end{array}$ & $\begin{array}{l}10.16 \\
11.27\end{array}$ & $\begin{array}{l}6.40 \\
7.13\end{array}$ & $\begin{array}{l}0.13 \\
0.11\end{array}$ \\
\hline \multirow[t]{3}{*}{ Black Creek near Vernon } & 01368950 & $\begin{array}{l}1975-76 \\
1976-77 \\
1977-78 \\
1978-79 \\
1979-80\end{array}$ & $\begin{array}{l}4.93 \\
4.29 \\
7.10 \\
6.20 \\
3.23\end{array}$ & $\begin{array}{l}2.64 \\
2.26 \\
3.84 \\
3.36 \\
1.74\end{array}$ & $\begin{array}{l}0.68 \\
0.65 \\
0.94 \\
0.80 \\
0.44\end{array}$ \\
\hline & & $\begin{array}{l}1980-81 \\
1981-82 \\
1982-83 \\
1983-84 \\
1984-85\end{array}$ & $\begin{array}{r}4.91 \\
9.53 \\
5.36 \\
10.38 \\
8.54\end{array}$ & $\begin{array}{l}2.67 \\
5.82 \\
3.38 \\
6.43 \\
5.36\end{array}$ & $\begin{array}{l}0.62 \\
0.36 \\
0.07 \\
0.27 \\
0.13\end{array}$ \\
\hline & & $\begin{array}{l}1985-86 \\
1986-87\end{array}$ & $\begin{array}{l}10.16 \\
11.27\end{array}$ & $\begin{array}{l}6.40 \\
7.13\end{array}$ & $\begin{array}{l}0.13 \\
0.11\end{array}$ \\
\hline
\end{tabular}


Table 14. Estimated road-salt application in the New Jersey drainage basins studied, in terms of chloride, sodium, and calcium by basin and winter season, 1975-76 through 1986-87--Continued

[Source: A.T. Woodrow, New Jersey Department of Transportation, written commun., 1989]

\begin{tabular}{|c|c|c|c|c|c|}
\hline \multicolumn{2}{|c|}{ Drainage basin } & \multirow[b]{2}{*}{$\begin{array}{l}\text { Winter } \\
\text { season }\end{array}$} & \multicolumn{3}{|c|}{ Application rate, in tons per square mile } \\
\hline Monitoring-station name & $\begin{array}{l}\text { Monitoring- } \\
\text { station number }\end{array}$ & & Chloride & Sodium & Calcium \\
\hline \multirow[t]{3}{*}{ Passaic River near Millington } & 01379000 & $\begin{array}{l}1975-76 \\
1976-77 \\
1977-78 \\
1978-79 \\
1979-80\end{array}$ & $\begin{array}{l}4.93 \\
4.29 \\
7.10 \\
6.20 \\
3.23\end{array}$ & $\begin{array}{l}2.64 \\
2.26 \\
3.84 \\
3.36 \\
1.74\end{array}$ & $\begin{array}{l}0.68 \\
0.65 \\
0.94 \\
0.80 \\
0.44\end{array}$ \\
\hline & & $\begin{array}{l}1980-81 \\
1981-82 \\
1982-83 \\
1983-84 \\
1984-85\end{array}$ & $\begin{array}{r}4.91 \\
9.53 \\
5.36 \\
10.38 \\
8.54\end{array}$ & $\begin{array}{l}2.67 \\
5.82 \\
3.38 \\
6.43 \\
5.36\end{array}$ & $\begin{array}{l}0.62 \\
0.36 \\
0.07 \\
0.27 \\
0.13\end{array}$ \\
\hline & & $\begin{array}{l}1985-86 \\
1986-87\end{array}$ & $\begin{array}{l}10.16 \\
11.27\end{array}$ & $\begin{array}{l}6.40 \\
7.13\end{array}$ & $\begin{array}{l}0.13 \\
0.11\end{array}$ \\
\hline \multirow[t]{3}{*}{ Passaic River near Chatham } & 01379500 & $\begin{array}{l}1975-76 \\
1976-77 \\
1977-78 \\
1978-79 \\
1979-80\end{array}$ & $\begin{array}{l}5.24 \\
4.63 \\
7.52 \\
6.55 \\
3.45\end{array}$ & $\begin{array}{l}2.81 \\
2.45 \\
4.07 \\
3.56 \\
1.85\end{array}$ & $\begin{array}{l}0.73 \\
0.68 \\
1.00 \\
0.84 \\
0.48\end{array}$ \\
\hline & & $\begin{array}{l}1980-81 \\
1981-82 \\
1982-83 \\
1983-84 \\
1984-85\end{array}$ & $\begin{array}{r}5.17 \\
9.86 \\
5.74 \\
10.90 \\
9.15\end{array}$ & $\begin{array}{l}2.81 \\
6.01 \\
3.62 \\
6.78 \\
5.75\end{array}$ & $\begin{array}{l}0.66 \\
0.39 \\
0.07 \\
0.26 \\
0.13\end{array}$ \\
\hline & & $\begin{array}{l}1985-86 \\
1986-87\end{array}$ & $\begin{array}{l}10.78 \\
11.92\end{array}$ & $\begin{array}{l}6.79 \\
7.54\end{array}$ & $\begin{array}{l}0.13 \\
0.11\end{array}$ \\
\hline \multirow[t]{3}{*}{ Rockaway River at Pine Brook } & 01381200 & $\begin{array}{l}1975-76 \\
1976-77 \\
1977-78 \\
1978-79 \\
1979-80\end{array}$ & $\begin{array}{l}4.93 \\
4.29 \\
7.10 \\
6.20 \\
3.23\end{array}$ & $\begin{array}{l}2.64 \\
2.26 \\
3.84 \\
3.36 \\
1.74\end{array}$ & $\begin{array}{l}0.68 \\
0.65 \\
0.94 \\
0.80 \\
0.44\end{array}$ \\
\hline & & $\begin{array}{l}1980-81 \\
1981-82 \\
1982-83 \\
1983-84 \\
1984-85\end{array}$ & $\begin{array}{r}4.91 \\
9.53 \\
5.36 \\
10.38 \\
8.54\end{array}$ & $\begin{array}{l}2.67 \\
5.82 \\
3.38 \\
6.43 \\
5.36\end{array}$ & $\begin{array}{l}0.62 \\
0.36 \\
0.07 \\
0.27 \\
0.13\end{array}$ \\
\hline & & $\begin{array}{l}1985-86 \\
1986-87\end{array}$ & $\begin{array}{l}10.16 \\
11.27\end{array}$ & $\begin{array}{l}6.40 \\
7.13\end{array}$ & $\begin{array}{l}0.13 \\
0.11\end{array}$ \\
\hline \multirow[t]{3}{*}{ Whippany River at Morristown } & 01381500 & $\begin{array}{l}1975-76 \\
1976-77 \\
1977-78 \\
1978-79 \\
1979-80\end{array}$ & $\begin{array}{l}4.93 \\
4.29 \\
7.10 \\
6.20 \\
3.23\end{array}$ & $\begin{array}{l}2.64 \\
2.26 \\
3.84 \\
3.36 \\
1.74\end{array}$ & $\begin{array}{l}0.68 \\
0.65 \\
0.94 \\
0.80 \\
0.44\end{array}$ \\
\hline & & $\begin{array}{l}1980-81 \\
1981-82 \\
1982-83 \\
1983-84 \\
1984-85\end{array}$ & $\begin{array}{r}4.91 \\
9.53 \\
5.36 \\
10.38 \\
8.54\end{array}$ & $\begin{array}{l}2.67 \\
5.82 \\
3.38 \\
6.43 \\
5.36\end{array}$ & $\begin{array}{l}0.62 \\
0.36 \\
0.07 \\
0.27 \\
0.13\end{array}$ \\
\hline & & $\begin{array}{l}1985-86 \\
1986-87\end{array}$ & $\begin{array}{l}10.16 \\
11.27\end{array}$ & $\begin{array}{l}6.40 \\
7.13\end{array}$ & $\begin{array}{l}0.13 \\
0.11\end{array}$ \\
\hline
\end{tabular}


Table 14. Estimated road-salt application in the New Jersey drainage basins studied, in terms of chloride, sodium, and calcium by basin and winter season, 1975-76 through 1986-87--Continued

[Source: A.T. Woodrow, New Jersey Department of Transportation, written commun., 1989]

\begin{tabular}{|c|c|c|c|c|c|}
\hline \multicolumn{2}{|c|}{ Drainage basin } & \multirow[b]{2}{*}{$\begin{array}{l}\text { Winter } \\
\text { season }\end{array}$} & \multicolumn{3}{|c|}{ Application rate, in tons per square mile } \\
\hline Monitoring-station name & $\begin{array}{l}\text { Monitoring- } \\
\text { station number }\end{array}$ & & Chloride & Sodium & Calcium \\
\hline \multirow[t]{3}{*}{ Whippany River near Pine Brook } & 01381800 & $\begin{array}{l}1975-76 \\
1976-77 \\
1977-78 \\
1978-79 \\
1979-80\end{array}$ & $\begin{array}{l}4.93 \\
4.29 \\
7.10 \\
6.20 \\
3.23\end{array}$ & $\begin{array}{l}2.64 \\
2.26 \\
3.84 \\
3.36 \\
1.74\end{array}$ & $\begin{array}{l}0.68 \\
0.65 \\
0.94 \\
0.80 \\
0.44\end{array}$ \\
\hline & & $\begin{array}{l}1980-81 \\
1981-82 \\
1982-83 \\
1983-84 \\
1984-85\end{array}$ & $\begin{array}{r}4.91 \\
9.53 \\
5.36 \\
10.38 \\
8.54\end{array}$ & $\begin{array}{l}2.67 \\
5.82 \\
3.38 \\
6.43 \\
5.36\end{array}$ & $\begin{array}{l}0.62 \\
0.36 \\
0.07 \\
0.27 \\
0.13\end{array}$ \\
\hline & & $\begin{array}{l}1985-86 \\
1986-87\end{array}$ & $\begin{array}{l}10.16 \\
11.27\end{array}$ & $\begin{array}{l}6.40 \\
7.13\end{array}$ & $\begin{array}{l}0.13 \\
0.11\end{array}$ \\
\hline \multirow[t]{3}{*}{ Passaic River at Two Bridges } & 01382000 & $\begin{array}{l}1975-76 \\
1976-77 \\
1977-78 \\
1978-79 \\
1979-80\end{array}$ & $\begin{array}{l}5.58 \\
4.99 \\
7.99 \\
6.93 \\
3.69\end{array}$ & $\begin{array}{l}2.99 \\
2.65 \\
4.32 \\
3.77 \\
1.98\end{array}$ & $\begin{array}{l}0.78 \\
0.73 \\
1.06 \\
0.89 \\
0.51\end{array}$ \\
\hline & & $\begin{array}{l}1980-81 \\
1981-82 \\
1982-83 \\
1983-84 \\
1984-85\end{array}$ & $\begin{array}{r}5.46 \\
10.23 \\
6.17 \\
11.49 \\
9.82\end{array}$ & $\begin{array}{l}2.97 \\
6.22 \\
3.89 \\
7.16 \\
6.18\end{array}$ & $\begin{array}{l}0.70 \\
0.43 \\
0.07 \\
0.25 \\
0.13\end{array}$ \\
\hline & & $\begin{array}{l}1985-86 \\
1986-87\end{array}$ & $\begin{array}{l}11.45 \\
12.63\end{array}$ & $\begin{array}{l}7.22 \\
7.99\end{array}$ & $\begin{array}{l}0.13 \\
0.11\end{array}$ \\
\hline \multirow[t]{3}{*}{ Wanaque River at Wanaque } & 01387000 & $\begin{array}{l}1975-76 \\
1976-77 \\
1977-78 \\
1978-79 \\
1979-80\end{array}$ & $\begin{array}{r}8.16 \\
7.72 \\
11.49 \\
9.81 \\
5.47\end{array}$ & $\begin{array}{l}4.36 \\
4.17 \\
6.21 \\
5.35 \\
2.91\end{array}$ & $\begin{array}{l}1.14 \\
1.02 \\
1.53 \\
1.24 \\
0.78\end{array}$ \\
\hline & & $\begin{array}{l}1980-81 \\
1981-82 \\
1982-83 \\
1983-84 \\
1984-85\end{array}$ & $\begin{array}{r}7.65 \\
12.97 \\
9.34 \\
15.84 \\
14.83\end{array}$ & $\begin{array}{r}4.14 \\
7.77 \\
5.91 \\
10.00 \\
9.37\end{array}$ & $\begin{array}{l}1.00 \\
0.69 \\
0.09 \\
0.17 \\
0.15\end{array}$ \\
\hline & & $\begin{array}{l}1985-86 \\
1986-87\end{array}$ & $\begin{array}{l}16.49 \\
17.96\end{array}$ & $\begin{array}{l}10.44 \\
11.39\end{array}$ & $\begin{array}{l}0.15 \\
0.12\end{array}$ \\
\hline \multirow[t]{3}{*}{ Saddle River at Lodi } & 01391500 & $\begin{array}{l}1975-76 \\
1976-77 \\
1977-78 \\
1978-79 \\
1979-80\end{array}$ & $\begin{array}{r}8.16 \\
7.72 \\
11.49 \\
9.81 \\
5.47\end{array}$ & $\begin{array}{l}4.36 \\
4.17 \\
6.21 \\
5.35 \\
2.91\end{array}$ & $\begin{array}{l}1.14 \\
1.02 \\
1.53 \\
1.24 \\
0.78\end{array}$ \\
\hline & & $\begin{array}{l}1980-81 \\
1981-82 \\
1982-83 \\
1983-84 \\
1984-85\end{array}$ & $\begin{array}{r}7.65 \\
12.97 \\
9.34 \\
15.84 \\
14.83\end{array}$ & $\begin{array}{r}4.14 \\
7.77 \\
5.91 \\
10.00 \\
9.37\end{array}$ & $\begin{array}{l}1.00 \\
0.69 \\
0.09 \\
0.17 \\
0.15\end{array}$ \\
\hline & & $\begin{array}{l}1985-86 \\
1986-87\end{array}$ & $\begin{array}{l}16.49 \\
17.96\end{array}$ & $\begin{array}{l}10.44 \\
11.39\end{array}$ & $\begin{array}{l}0.15 \\
0.12\end{array}$ \\
\hline
\end{tabular}


Table 14. Estimated road-salt application in the New Jersey drainage basins studied, in terms of chloride, sodium, and calcium by basin and winter season, 1975-76 through 1986-87--Continued

[Source: A.T. Woodrow, New Jersey Department of Transportation, written commun., 1989]

\begin{tabular}{|c|c|c|c|c|c|}
\hline \multicolumn{2}{|c|}{ Drainage basin } & \multirow[b]{2}{*}{$\begin{array}{l}\text { Winter } \\
\text { season }\end{array}$} & \multicolumn{3}{|c|}{ Application rate, in tons per square mile } \\
\hline Monitoring-station name & $\begin{array}{l}\text { Monitoring- } \\
\text { station number }\end{array}$ & & Chloride & Sodium & Calcium \\
\hline \multirow[t]{3}{*}{ SB Raritan River at Middle Valley } & 01396280 & $\begin{array}{l}1975-76 \\
1976-77 \\
1977-78 \\
1978-79 \\
1979-80\end{array}$ & $\begin{array}{l}4.93 \\
4.29 \\
7.10 \\
6.20 \\
3.23\end{array}$ & $\begin{array}{l}2.64 \\
2.26 \\
3.84 \\
3.36 \\
1.74\end{array}$ & $\begin{array}{l}0.68 \\
0.65 \\
0.94 \\
0.80 \\
0.44\end{array}$ \\
\hline & & $\begin{array}{l}1980-81 \\
1981-82 \\
1982-83 \\
1983-84 \\
1984-85\end{array}$ & $\begin{array}{r}4.91 \\
9.53 \\
5.36 \\
10.38 \\
8.54\end{array}$ & $\begin{array}{l}2.67 \\
5.82 \\
3.38 \\
6.43 \\
5.36\end{array}$ & $\begin{array}{l}0.62 \\
0.36 \\
0.07 \\
0.27 \\
0.13\end{array}$ \\
\hline & & $\begin{array}{l}1985-86 \\
1986-87\end{array}$ & $\begin{array}{l}10.16 \\
11.27\end{array}$ & $\begin{array}{l}6.40 \\
7.13\end{array}$ & $\begin{array}{l}0.13 \\
0.11\end{array}$ \\
\hline \multirow[t]{3}{*}{ SB Raritan River at High Bridge } & 01396535 & $\begin{array}{l}1975-76 \\
1976-77 \\
1977-78 \\
1978-79 \\
1979-80\end{array}$ & $\begin{array}{l}4.93 \\
4.29 \\
7.10 \\
6.20 \\
3.23\end{array}$ & $\begin{array}{l}2.64 \\
2.26 \\
3.84 \\
3.36 \\
1.74\end{array}$ & $\begin{array}{l}0.68 \\
0.65 \\
0.94 \\
0.80 \\
0.44\end{array}$ \\
\hline & & $\begin{array}{l}1980-81 \\
1981-82 \\
1982-83 \\
1983-84 \\
1984-85\end{array}$ & $\begin{array}{r}4.91 \\
9.53 \\
5.36 \\
10.38 \\
8.54\end{array}$ & $\begin{array}{l}2.67 \\
5.82 \\
3.38 \\
6.43 \\
5.36\end{array}$ & $\begin{array}{l}0.62 \\
0.36 \\
0.07 \\
0.27 \\
0.13\end{array}$ \\
\hline & & $\begin{array}{l}1985-86 \\
1986-87\end{array}$ & $\begin{array}{l}10.16 \\
11.27\end{array}$ & $\begin{array}{l}6.40 \\
7.13\end{array}$ & $\begin{array}{l}0.13 \\
0.11\end{array}$ \\
\hline \multirow[t]{3}{*}{ Mulhockaway Creek at Van Syckel } & 01396660 & $\begin{array}{l}1975-76 \\
1976-77 \\
1977-78 \\
1978-79 \\
1979-80\end{array}$ & $\begin{array}{l}4.93 \\
4.29 \\
7.10 \\
6.20 \\
3.23\end{array}$ & $\begin{array}{l}2.64 \\
2.26 \\
3.84 \\
3.36 \\
1.74\end{array}$ & $\begin{array}{l}0.68 \\
0.65 \\
0.94 \\
0.80 \\
0.44\end{array}$ \\
\hline & & $\begin{array}{c}1980-81 \\
1981-82 \\
1982-83 \\
1983-84 \\
1984-85\end{array}$ & $\begin{array}{r}4.91 \\
9.53 \\
5.36 \\
10.38 \\
8.54\end{array}$ & $\begin{array}{l}2.67 \\
5.82 \\
3.38 \\
6.43 \\
5.36\end{array}$ & $\begin{array}{l}0.62 \\
0.36 \\
0.07 \\
0.27 \\
0.13\end{array}$ \\
\hline & & $\begin{array}{l}1985-86 \\
1986-87\end{array}$ & $\begin{array}{l}10.16 \\
11.27\end{array}$ & $\begin{array}{l}6.40 \\
7.13\end{array}$ & $\begin{array}{l}0.13 \\
0.11\end{array}$ \\
\hline \multirow[t]{3}{*}{ SB Raritan River at Three Bridges } & 01397400 & $\begin{array}{l}1975-76 \\
1976-77 \\
1977-78 \\
1978-79 \\
1979-80\end{array}$ & $\begin{array}{l}4.93 \\
4.29 \\
7.10 \\
6.20 \\
3.23\end{array}$ & $\begin{array}{l}2.64 \\
2.26 \\
3.84 \\
3.36 \\
1.74\end{array}$ & $\begin{array}{l}0.68 \\
0.65 \\
0.94 \\
0.80 \\
0.44\end{array}$ \\
\hline & & $\begin{array}{l}1980-81 \\
1981-82 \\
1982-83 \\
1983-84 \\
1984-85\end{array}$ & $\begin{array}{r}4.91 \\
9.53 \\
5.36 \\
10.38 \\
8.54\end{array}$ & $\begin{array}{l}2.67 \\
5.82 \\
3.38 \\
6.43 \\
5.36\end{array}$ & $\begin{array}{l}0.62 \\
0.36 \\
0.07 \\
0.27 \\
0.13\end{array}$ \\
\hline & & $\begin{array}{l}1985-86 \\
1986-87\end{array}$ & $\begin{array}{l}10.16 \\
11.27\end{array}$ & $\begin{array}{l}6.40 \\
7.13\end{array}$ & $\begin{array}{l}0.13 \\
0.11\end{array}$ \\
\hline
\end{tabular}


Table I4. Estimated road-salt application in the New Jersey drainage basins studied, in terms of chloride, sodium, and calcium by basin and winter season, 1975-76 through I986-87--Continued

[Source: A.T. Woodrow, New Jersey Department of Transportation, written commun., 1989]

\begin{tabular}{|c|c|c|c|c|c|}
\hline \multicolumn{2}{|c|}{ Drainage basin } & \multirow[b]{2}{*}{$\begin{array}{l}\text { Winter } \\
\text { season }\end{array}$} & \multicolumn{3}{|c|}{ Application rate, in tons per square mile } \\
\hline Monitoring-station name & $\begin{array}{l}\text { Monitoring- } \\
\text { station number }\end{array}$ & & Chloride & Sodium & Calcium \\
\hline \multirow[t]{3}{*}{ NB Raritan River near Chester } & 01398260 & $\begin{array}{l}1975-76 \\
1976-77 \\
1977-78 \\
1978-79 \\
1979-80\end{array}$ & $\begin{array}{l}4.93 \\
4.29 \\
7.10 \\
6.20 \\
3.23\end{array}$ & $\begin{array}{l}2.64 \\
2.26 \\
3.84 \\
3.36 \\
1.74\end{array}$ & $\begin{array}{l}0.68 \\
0.65 \\
0.94 \\
0.80 \\
0.44\end{array}$ \\
\hline & & $\begin{array}{l}1980-81 \\
1981-82 \\
1982-83 \\
1983-84 \\
1984-85\end{array}$ & $\begin{array}{r}4.91 \\
9.53 \\
5.36 \\
10.38 \\
8.54\end{array}$ & $\begin{array}{l}2.67 \\
5.82 \\
3.38 \\
6.43 \\
5.36\end{array}$ & $\begin{array}{l}0.62 \\
0.36 \\
0.07 \\
0.27 \\
0.13\end{array}$ \\
\hline & & $\begin{array}{l}1985-86 \\
1986-87\end{array}$ & $\begin{array}{l}10.16 \\
11.27\end{array}$ & $\begin{array}{l}6.40 \\
7.13\end{array}$ & $\begin{array}{l}0.13 \\
0.11\end{array}$ \\
\hline \multirow[t]{3}{*}{ NB Raritan River at Burnt Mills } & 01399120 & $\begin{array}{l}1975-76 \\
1976-77 \\
1977-78 \\
1978-79 \\
1979-80\end{array}$ & $\begin{array}{l}4.93 \\
4.29 \\
7.10 \\
6.20 \\
3.23\end{array}$ & $\begin{array}{l}2.64 \\
2.26 \\
3.84 \\
3.36 \\
1.74\end{array}$ & $\begin{array}{l}0.68 \\
0.65 \\
0.94 \\
0.80 \\
0.44\end{array}$ \\
\hline & & $\begin{array}{l}1980-81 \\
1981-82 \\
1982-83 \\
1983-84 \\
1984-85\end{array}$ & $\begin{array}{r}4.91 \\
9.53 \\
5.36 \\
10.38 \\
8.54\end{array}$ & $\begin{array}{l}2.67 \\
5.82 \\
3.38 \\
6.43 \\
5.36\end{array}$ & $\begin{array}{l}0.62 \\
0.36 \\
0.07 \\
0.27 \\
0.13\end{array}$ \\
\hline & & $\begin{array}{l}1985-86 \\
1986-87\end{array}$ & $\begin{array}{l}10.16 \\
11.27\end{array}$ & $\begin{array}{l}6.40 \\
7.13\end{array}$ & $\begin{array}{l}0.13 \\
0.11\end{array}$ \\
\hline \multirow[t]{3}{*}{ Rockaway Creek at Whitehouse } & 01399700 & $\begin{array}{l}1975-76 \\
1976-77 \\
1977-78 \\
1978-79 \\
1979-80\end{array}$ & $\begin{array}{l}4.93 \\
4.29 \\
7.10 \\
6.20 \\
3.23\end{array}$ & $\begin{array}{l}2.64 \\
2.26 \\
3.84 \\
3.36 \\
1.74\end{array}$ & $\begin{array}{l}0.68 \\
0.65 \\
0.94 \\
0.80 \\
0.44\end{array}$ \\
\hline & & $\begin{array}{l}1980-81 \\
1981-82 \\
1982-83 \\
1983-84 \\
1984-85\end{array}$ & $\begin{array}{r}4.91 \\
9.53 \\
5.36 \\
10.38 \\
8.54\end{array}$ & $\begin{array}{l}2.67 \\
5.82 \\
3.38 \\
6.43 \\
5.36\end{array}$ & $\begin{array}{l}0.62 \\
0.36 \\
0.07 \\
0.27 \\
0.13\end{array}$ \\
\hline & & $\begin{array}{l}1985-86 \\
1986-87\end{array}$ & $\begin{array}{l}10.16 \\
11.27\end{array}$ & $\begin{array}{l}6.40 \\
7.13\end{array}$ & $\begin{array}{l}0.13 \\
0.11\end{array}$ \\
\hline \multirow[t]{3}{*}{ Lamington River at Burnt Mills } & 01399780 & $\begin{array}{l}1975-76 \\
1976-77 \\
1977-78 \\
1978-79 \\
1979-80\end{array}$ & $\begin{array}{l}4.93 \\
4.29 \\
7.10 \\
6.20 \\
3.23\end{array}$ & $\begin{array}{l}2.64 \\
2.26 \\
3.84 \\
3.36 \\
1.74\end{array}$ & $\begin{array}{l}0.68 \\
0.65 \\
0.94 \\
0.80 \\
0.44\end{array}$ \\
\hline & & $\begin{array}{l}1980-81 \\
1981-82 \\
1982-83 \\
1983-84 \\
1984-85\end{array}$ & $\begin{array}{r}4.91 \\
9.53 \\
5.36 \\
10.38 \\
8.54\end{array}$ & $\begin{array}{l}2.67 \\
5.82 \\
3.38 \\
6.43 \\
5.36\end{array}$ & $\begin{array}{l}0.62 \\
0.36 \\
0.07 \\
0.27 \\
0.13\end{array}$ \\
\hline & & $\begin{array}{l}1985-86 \\
1986-87\end{array}$ & $\begin{array}{l}10.16 \\
11.27\end{array}$ & $\begin{array}{l}6.40 \\
7.13\end{array}$ & $\begin{array}{l}0.13 \\
0.11\end{array}$ \\
\hline
\end{tabular}


Table I4. Estimated road-salt application in the New Jersey drainage basins studied, in terms of chloride, sodium, and calcium by basin and winter season, I975-76 through I986-87--Continued

[Source: A.T. Woodrow, New Jersey Department of Transportation, written commun., 1989]

\begin{tabular}{|c|c|c|c|c|c|}
\hline \multicolumn{2}{|c|}{ Drainage basin } & \multirow[b]{2}{*}{$\begin{array}{l}\text { Winter } \\
\text { season }\end{array}$} & \multicolumn{3}{|c|}{ Application rate, in tons per square mile } \\
\hline Monitoring-station name & $\begin{array}{l}\text { Monitoring- } \\
\text { station number }\end{array}$ & & Chloride & Sodium & Calcium \\
\hline \multirow[t]{3}{*}{ Raritan River at Manville } & 01400500 & $\begin{array}{l}1975-76 \\
1976-77 \\
1977-78 \\
1978-79 \\
1979-80\end{array}$ & $\begin{array}{l}4.93 \\
4.29 \\
7.10 \\
6.20 \\
3.23\end{array}$ & $\begin{array}{l}2.64 \\
2.26 \\
3.84 \\
3.36 \\
1.74\end{array}$ & $\begin{array}{l}0.68 \\
0.65 \\
0.94 \\
0.80 \\
0.44\end{array}$ \\
\hline & & $\begin{array}{l}1980-81 \\
1981-82 \\
1982-83 \\
1983-84 \\
1984-85\end{array}$ & $\begin{array}{r}4.91 \\
9.53 \\
5.36 \\
10.38 \\
8.54\end{array}$ & $\begin{array}{l}2.67 \\
5.82 \\
3.38 \\
6.43 \\
5.36\end{array}$ & $\begin{array}{l}0.62 \\
0.36 \\
0.07 \\
0.27 \\
0.13\end{array}$ \\
\hline & & $\begin{array}{l}1985-86 \\
1986-87\end{array}$ & $\begin{array}{l}10.16 \\
11.27\end{array}$ & $\begin{array}{l}6.40 \\
7.13\end{array}$ & $\begin{array}{l}0.13 \\
0.11\end{array}$ \\
\hline \multirow[t]{3}{*}{ Millstone River at Grovers Mill } & 01400650 & $\begin{array}{l}1975-76 \\
1976-77 \\
1977-78 \\
1978-79 \\
1979-80\end{array}$ & $\begin{array}{l}4.23 \\
3.73 \\
4.60 \\
3.88 \\
2.23\end{array}$ & $\begin{array}{l}2.52 \\
2.12 \\
2.55 \\
2.11 \\
1.23\end{array}$ & $\begin{array}{l}0.25 \\
0.36 \\
0.52 \\
0.49 \\
0.26\end{array}$ \\
\hline & & $\begin{array}{l}1980-81 \\
1981-82 \\
1982-83 \\
1983-84 \\
1984-85\end{array}$ & $\begin{array}{l}2.94 \\
5.04 \\
2.58 \\
4.93 \\
3.89\end{array}$ & $\begin{array}{l}1.63 \\
3.01 \\
1.62 \\
3.12 \\
2.39\end{array}$ & $\begin{array}{l}0.33 \\
0.28 \\
0.05 \\
0.05 \\
0.13\end{array}$ \\
\hline & & $\begin{array}{l}1985-86 \\
1986-87\end{array}$ & $\begin{array}{l}4.53 \\
7.43\end{array}$ & $\begin{array}{l}2.86 \\
4.70\end{array}$ & $\begin{array}{l}0.05 \\
0.07\end{array}$ \\
\hline \multirow[t]{3}{*}{ Millstone River at Kingston } & 01401440 & $\begin{array}{l}1975-76 \\
1976-77 \\
1977-78 \\
1978-79 \\
1979-80\end{array}$ & $\begin{array}{l}4.26 \\
3.76 \\
4.71 \\
3.99 \\
2.27\end{array}$ & $\begin{array}{l}2.53 \\
2.12 \\
2.61 \\
2.17 \\
1.25\end{array}$ & $\begin{array}{l}0.27 \\
0.38 \\
0.54 \\
0.51 \\
0.27\end{array}$ \\
\hline & & $\begin{array}{l}1980-81 \\
1981-82 \\
1982-83 \\
1983-84 \\
1984-85\end{array}$ & $\begin{array}{l}3.03 \\
5.25 \\
2.71 \\
5.18 \\
4.10\end{array}$ & $\begin{array}{l}1.68 \\
3.14 \\
1.70 \\
3.27 \\
2.53\end{array}$ & $\begin{array}{l}0.35 \\
0.28 \\
0.05 \\
0.06 \\
0.13\end{array}$ \\
\hline & & $\begin{array}{l}1985-86 \\
1986-87\end{array}$ & $\begin{array}{l}4.79 \\
7.60\end{array}$ & $\begin{array}{l}3.02 \\
4.81\end{array}$ & $\begin{array}{l}0.05 \\
0.07\end{array}$ \\
\hline \multirow[t]{3}{*}{ Beden Brook near Rocky Hill } & 01401600 & $\begin{array}{l}1975-76 \\
1976-77 \\
1977-78 \\
1978-79 \\
1979-80\end{array}$ & $\begin{array}{l}4.70 \\
4.11 \\
6.27 \\
5.43 \\
2.90\end{array}$ & $\begin{array}{l}2.60 \\
2.21 \\
3.41 \\
2.95 \\
1.57\end{array}$ & $\begin{array}{l}0.54 \\
0.55 \\
0.80 \\
0.70 \\
0.38\end{array}$ \\
\hline & & $\begin{array}{l}1980-81 \\
1981-82 \\
1982-83 \\
1983-84 \\
1984-85\end{array}$ & $\begin{array}{l}4.26 \\
8.05 \\
4.44 \\
8.58 \\
7.01\end{array}$ & $\begin{array}{l}2.33 \\
4.90 \\
2.80 \\
5.34 \\
4.38\end{array}$ & $\begin{array}{l}0.53 \\
0.33 \\
0.06 \\
0.20 \\
0.13\end{array}$ \\
\hline & & $\begin{array}{l}1985-86 \\
1986-87\end{array}$ & $\begin{array}{r}8.31 \\
10.00\end{array}$ & $\begin{array}{l}5.23 \\
6.32\end{array}$ & $\begin{array}{l}0.10 \\
0.09\end{array}$ \\
\hline
\end{tabular}


Table 14. Estimated road-salt application in the New Jersey drainage basins studied, in terms of chloride, sodium, and calcium by basin and winter season, 1975-76 through 1986-87--Continued

[Source: A.T. Woodrow, New Jersey Department of Transportation, written commun., I989]

\begin{tabular}{|c|c|c|c|c|c|}
\hline \multicolumn{2}{|c|}{ Drainage basin } & \multirow[b]{2}{*}{$\begin{array}{l}\text { Winter } \\
\text { season }\end{array}$} & \multicolumn{3}{|c|}{ Application rate, in tons per square mile } \\
\hline Monitoring-station name & $\begin{array}{l}\text { Monitoring- } \\
\text { station number }\end{array}$ & & Chloride & Sodium & Calcium \\
\hline \multirow[t]{12}{*}{ Millstone River at Weston } & 01402540 & $1975-76$ & 4.43 & 2.56 & 0.37 \\
\hline & & $1976-77$ & 3.90 & 2.16 & 0.45 \\
\hline & & $1977-78$ & 5.33 & 2.93 & 0.64 \\
\hline & & $1978-79$ & 4.56 & 2.48 & 0.59 \\
\hline & & $1979-80$ & 2.52 & 1.38 & 0.31 \\
\hline & & $1980-81$ & 3.51 & 1.93 & 0.42 \\
\hline & & $1981-82$ & 6.35 & 3.83 & 0.31 \\
\hline & & $1982-83$ & 3.39 & 2.13 & 0.05 \\
\hline & & $1983-84$ & 6.52 & 4.08 & 0.12 \\
\hline & & $1984-85$ & 5.24 & 3.26 & 0.13 \\
\hline & & $1985-86$ & 6.17 & 3.89 & 0.07 \\
\hline & & $1986-87$ & 8.55 & 5.41 & 0.08 \\
\hline \multirow{11}{*}{\multicolumn{2}{|c|}{ Manalapan Brook at Federal Rd near Manalapan 01405340}} & $1975-76$ & 4.23 & 2.52 & 0.25 \\
\hline & & $1976-77$ & 3.73 & 2.12 & 0.36 \\
\hline & & $1977-78$ & 4.60 & 2.55 & 0.52 \\
\hline & & $\begin{array}{l}1978-79 \\
1979-80\end{array}$ & $\begin{array}{l}3.88 \\
2.23\end{array}$ & $\begin{array}{l}2.11 \\
1.23\end{array}$ & $\begin{array}{l}0.49 \\
0.26\end{array}$ \\
\hline & & & & & \\
\hline & & $\begin{array}{l}1980-81 \\
1981-82\end{array}$ & $\begin{array}{l}2.94 \\
5.04\end{array}$ & $\begin{array}{l}1.63 \\
3.01\end{array}$ & $\begin{array}{l}0.33 \\
0.28\end{array}$ \\
\hline & & $1982-83$ & 2.58 & 1.62 & 0.05 \\
\hline & & $1983-84$ & 4.93 & 3.12 & 0.05 \\
\hline & & $1984-85$ & 3.89 & 2.39 & 0.13 \\
\hline & & $1985-86$ & 4.53 & 2.86 & 0.05 \\
\hline & & $1986-87$ & 7.43 & 4.70 & 0.07 \\
\hline \multirow[t]{12}{*}{ Shark River near Neptune City } & 01407705 & $1975-76$ & 4.23 & 2.52 & 0.25 \\
\hline & & $1976-77$ & 3.73 & 2.12 & 0.36 \\
\hline & & $1977-78$ & 4.60 & 2.55 & 0.52 \\
\hline & & $1978-79$ & 3.88 & 2.11 & 0.49 \\
\hline & & $1979-80$ & 2.23 & 1.23 & 0.26 \\
\hline & & $1980-81$ & 2.94 & 1.63 & 0.33 \\
\hline & & $1981-82$ & 5.04 & 3.01 & 0.28 \\
\hline & & $1982-83$ & 2.58 & 1.62 & 0.05 \\
\hline & & $1983-84$ & 4.93 & 3.12 & 0.05 \\
\hline & & $1984-85$ & 3.89 & 2.39 & 0.13 \\
\hline & & $1985-86$ & 4.53 & 2.86 & 0.05 \\
\hline & & $1986-87$ & 7.43 & 4.70 & 0.07 \\
\hline \multirow[t]{11}{*}{ Jumping Brook near Neptune City } & 01407760 & $1975-76$ & 4.23 & 2.52 & 0.25 \\
\hline & & $1976-77$ & 3.73 & 2.12 & 0.36 \\
\hline & & $\begin{array}{l}1977-78 \\
1978-79\end{array}$ & $\begin{array}{l}4.60 \\
3.88\end{array}$ & $\begin{array}{l}2.55 \\
2.11\end{array}$ & $\begin{array}{l}0.52 \\
0.49\end{array}$ \\
\hline & & $1979-80$ & 2.23 & 1.23 & 0.26 \\
\hline & & 1980-81 & 2.94 & 1.63 & 0.33 \\
\hline & & $1981-82$ & 5.04 & 3.01 & 0.28 \\
\hline & & $1982-83$ & 2.58 & 1.62 & 0.05 \\
\hline & & $1983-84$ & 4.93 & 3.12 & 0.05 \\
\hline & & $1984-85$ & 3.89 & 2.39 & 0.13 \\
\hline & & $1985-86$ & 4.53 & 2.86 & 0.05 \\
\hline & & $1986-87$ & 7.43 & 4.70 & 0.07 \\
\hline
\end{tabular}


Table 14. Estimated road-salt application in the New Jersey drainage basins studied, in terms of chloride, sodium, and calcium by basin and winter season, 1975-76 through 1986-87--Continued

[Source: A.T. Woodrow, New Jersey Department of Transportation, written commun., 1989]

\begin{tabular}{|c|c|c|c|c|c|}
\hline \multicolumn{2}{|l|}{ Drainage basin } & \multirow[b]{2}{*}{$\begin{array}{l}\text { Winter } \\
\text { season }\end{array}$} & \multicolumn{3}{|c|}{ Application rate, in tons per square mile } \\
\hline Monitoring-station name & $\begin{array}{c}\text { Monitoring- } \\
\text { station number }\end{array}$ & & Chloride & Sodium & Calcium \\
\hline \multirow[t]{3}{*}{ Marsh Bog Brook at Squankum } & 01407997 & $\begin{array}{l}1975-76 \\
1976-77 \\
1977-78 \\
1978-79 \\
1979-80\end{array}$ & $\begin{array}{l}4.23 \\
3.73 \\
4.60 \\
3.88 \\
2.23\end{array}$ & $\begin{array}{l}2.52 \\
2.12 \\
2.55 \\
2.11 \\
1.23\end{array}$ & $\begin{array}{l}0.25 \\
0.36 \\
0.52 \\
0.49 \\
0.26\end{array}$ \\
\hline & & $\begin{array}{l}1980-81 \\
1981-82 \\
1982-83 \\
1983-84 \\
1984-85\end{array}$ & $\begin{array}{l}2.94 \\
5.04 \\
2.58 \\
4.93 \\
3.89\end{array}$ & $\begin{array}{l}1.63 \\
3.01 \\
1.62 \\
3.12 \\
2.39\end{array}$ & $\begin{array}{l}0.33 \\
0.28 \\
0.05 \\
0.05 \\
0.13\end{array}$ \\
\hline & & $\begin{array}{l}1985-86 \\
1986-87\end{array}$ & $\begin{array}{l}4.53 \\
7.43\end{array}$ & $\begin{array}{l}2.86 \\
4.70\end{array}$ & $\begin{array}{l}0.05 \\
0.07\end{array}$ \\
\hline \multirow[t]{3}{*}{ Toms River near Toms River } & 01408500 & $\begin{array}{l}1975-76 \\
1976-77 \\
1977-78 \\
1978-79 \\
1979-80\end{array}$ & $\begin{array}{l}4.23 \\
3.73 \\
4.60 \\
3.88 \\
2.23\end{array}$ & $\begin{array}{l}2.52 \\
2.12 \\
2.55 \\
2.11 \\
1.23\end{array}$ & $\begin{array}{l}0.25 \\
0.36 \\
0.52 \\
0.49 \\
0.26\end{array}$ \\
\hline & & $\begin{array}{l}1980-81 \\
1981-82 \\
1982-83 \\
1983-84 \\
1984-85\end{array}$ & $\begin{array}{l}2.94 \\
5.04 \\
2.58 \\
4.93 \\
3.89\end{array}$ & $\begin{array}{l}1.63 \\
3.01 \\
1.62 \\
3.12 \\
2.39\end{array}$ & $\begin{array}{l}0.33 \\
0.28 \\
0.05 \\
0.05 \\
0.13\end{array}$ \\
\hline & & $\begin{array}{l}1985-86 \\
1986-87\end{array}$ & $\begin{array}{l}4.53 \\
7.43\end{array}$ & $\begin{array}{l}2.86 \\
4.70\end{array}$ & $\begin{array}{l}0.05 \\
0.07\end{array}$ \\
\hline \multirow[t]{3}{*}{ Mullica River at outlet of Atsion Lake at Atsion } & 01409387 & $\begin{array}{l}1975-76 \\
1976-77 \\
1977-78 \\
1978-79 \\
1979-80\end{array}$ & $\begin{array}{l}0.95 \\
1.18 \\
1.40 \\
1.47 \\
0.99\end{array}$ & $\begin{array}{l}0.56 \\
0.65 \\
0.75 \\
0.79 \\
0.54\end{array}$ & $\begin{array}{l}0.06 \\
0.13 \\
0.19 \\
0.20 \\
0.12\end{array}$ \\
\hline & & $\begin{array}{l}1980-81 \\
1981-82 \\
1982-83 \\
1983-84 \\
1984-85\end{array}$ & $\begin{array}{l}0.82 \\
1.44 \\
0.86 \\
1.51 \\
1.81\end{array}$ & $\begin{array}{l}0.46 \\
0.84 \\
0.48 \\
0.92 \\
1.14\end{array}$ & $\begin{array}{l}0.09 \\
0.10 \\
0.10 \\
0.06 \\
0.03\end{array}$ \\
\hline & & $\begin{array}{l}1985-86 \\
1986-87\end{array}$ & $\begin{array}{l}2.54 \\
3.14\end{array}$ & $\begin{array}{l}1.59 \\
1.98\end{array}$ & $\begin{array}{l}0.04 \\
0.04\end{array}$ \\
\hline \multirow[t]{3}{*}{ Hammonton Creek at Westcoatville } & 01409416 & $\begin{array}{l}1975-76 \\
1976-77 \\
1977-78 \\
1978-79 \\
1979-80\end{array}$ & $\begin{array}{l}0.95 \\
1.18 \\
1.40 \\
1.47 \\
0.99\end{array}$ & $\begin{array}{l}0.56 \\
0.65 \\
0.75 \\
0.79 \\
0.54\end{array}$ & $\begin{array}{l}0.06 \\
0.13 \\
0.19 \\
0.20 \\
0.12\end{array}$ \\
\hline & & $\begin{array}{l}1980-81 \\
1981-82 \\
1982-83 \\
1983-84 \\
1984-85\end{array}$ & $\begin{array}{l}0.82 \\
1.44 \\
0.86 \\
1.51 \\
1.81\end{array}$ & $\begin{array}{l}0.46 \\
0.84 \\
0.48 \\
0.92 \\
1.14\end{array}$ & $\begin{array}{l}0.09 \\
0.10 \\
0.10 \\
0.06 \\
0.03\end{array}$ \\
\hline & & $\begin{array}{l}1985-86 \\
1986-87\end{array}$ & $\begin{array}{l}2.54 \\
3.14\end{array}$ & $\begin{array}{l}1.59 \\
1.98\end{array}$ & $\begin{array}{l}0.04 \\
0.04\end{array}$ \\
\hline
\end{tabular}


Table 14. Estimated road-salt application in the New Jersey drainage basins studied, in terms of chloride, sodium, and calcium by basin and winter season, 1975-76 through I986-87--Continued

[Source: A.T. Woodrow, New Jersey Department of Transportation, written commun., I989]

\begin{tabular}{|c|c|c|c|c|c|}
\hline \multicolumn{2}{|l|}{ Drainage basin } & \multirow[b]{2}{*}{$\begin{array}{l}\text { Winter } \\
\text { season }\end{array}$} & \multicolumn{3}{|c|}{ Application rate, in tons per square mile } \\
\hline Monitoring-station name & $\begin{array}{c}\text { Monitoring- } \\
\text { station number }\end{array}$ & & Chloride & Sodium & Calcium \\
\hline \multirow[t]{11}{*}{ Batsto River at Batsto } & 01409500 & $1975-76$ & 0.95 & 0.56 & 0.06 \\
\hline & & $1976-77$ & 1.18 & 0.65 & 0.13 \\
\hline & & $1977-78$ & 1.40 & 0.75 & 0.19 \\
\hline & & $\begin{array}{l}1978-79 \\
1979-80\end{array}$ & $\begin{array}{l}1.47 \\
0.99\end{array}$ & $\begin{array}{l}0.79 \\
0.54\end{array}$ & $\begin{array}{l}0.20 \\
0.12\end{array}$ \\
\hline & & & & & \\
\hline & & $1981-82$ & 0.02 & 0.40 & 0.10 \\
\hline & & $1982-83$ & 0.86 & 0.48 & 0.10 \\
\hline & & 1983-84 & 1.51 & 0.92 & 0.06 \\
\hline & & 1984-85 & 1.81 & 1.14 & 0.03 \\
\hline & & $1985-86$ & 2.54 & 1.59 & 0.04 \\
\hline & & $1986-87$ & 3.14 & 1.98 & 0.04 \\
\hline \multirow[t]{12}{*}{ West Branch Wading River at Maxwell } & 01409815 & $1975-76$ & 0.96 & 0.57 & 0.06 \\
\hline & & $1976-77$ & 1.19 & 0.66 & 0.13 \\
\hline & & $1977-78$ & 1.41 & 0.76 & 0.19 \\
\hline & & $1978-79$ & 1.48 & 0.80 & 0.20 \\
\hline & & $1979-80$ & 0.99 & 0.54 & 0.12 \\
\hline & & $1980-81$ & 0.83 & 0.47 & 0.09 \\
\hline & & $1981-82$ & 1.45 & 0.85 & 0.10 \\
\hline & & $1982-83$ & 0.87 & 0.48 & 0.10 \\
\hline & & $1983-84$ & 1.53 & 0.93 & 0.06 \\
\hline & & $1984-85$ & 1.82 & 1.14 & 0.03 \\
\hline & & $1985-86$ & 2.55 & 1.60 & 0.04 \\
\hline & & $1986-87$ & 3.16 & 1.99 & 0.04 \\
\hline \multirow[t]{12}{*}{ Oswego River at Harrisville } & 01410000 & $1975-76$ & 2.21 & 1.32 & 0.13 \\
\hline & & 1976-77 & 2.17 & 1.22 & 0.22 \\
\hline & & $1977-78$ & 2.64 & 1.45 & 0.32 \\
\hline & & $1978-79$ & 2.41 & 1.30 & 0.32 \\
\hline & & $1979-80$ & 1.47 & 0.81 & 0.18 \\
\hline & & $1980-81$ & 1.64 & 0.91 & 0.18 \\
\hline & & $1981-82$ & 2.83 & 1.68 & 0.17 \\
\hline & & $1982-83$ & 1.53 & 0.92 & 0.08 \\
\hline & & $1983-84$ & 2.83 & 1.77 & 0.05 \\
\hline & & $1984-85$ & 2.62 & 1.62 & 0.06 \\
\hline & & $1985-86$ & 3.31 & 2.08 & 0.04 \\
\hline & & $1986-87$ & 4.80 & 3.03 & 0.05 \\
\hline \multirow[t]{12}{*}{ East Branch Bass River near New Gretna } & 01410150 & $1975-76$ & 1.14 & 0.68 & 0.07 \\
\hline & & $1976-77$ & 1.33 & 0.74 & 0.15 \\
\hline & & $1977-78$ & 1.59 & 0.86 & 0.21 \\
\hline & & $1978-79$ & 1.61 & 0.87 & 0.22 \\
\hline & & $1979-80$ & 1.06 & 0.58 & 0.13 \\
\hline & & $1980-81$ & 0.95 & 0.53 & 0.10 \\
\hline & & $1981-82$ & 1.65 & 0.97 & 0.11 \\
\hline & & $1982-83$ & 0.96 & 0.54 & 0.10 \\
\hline & & 1983-84 & 1.71 & 1.05 & 0.06 \\
\hline & & $1984-85$ & 1.93 & 1.21 & 0.03 \\
\hline & & $1985-86$ & 2.65 & 1.67 & 0.04 \\
\hline & & 1986-87 & 3.39 & 2.14 & 0.04 \\
\hline
\end{tabular}


Table 14. Estimated road-salt application in the New Jersey drainage basins studied, in terms of chloride, sodium, and calcium by basin and winter season, 1975-76 through 1986-87--Continued

[Source: A.T. Woodrow, New Jersey Department of Transportation, written commun., 1989]

\begin{tabular}{|c|c|c|c|c|c|}
\hline \multicolumn{2}{|c|}{ Drainage basin } & \multirow[b]{2}{*}{$\begin{array}{l}\text { Winter } \\
\text { season }\end{array}$} & \multicolumn{3}{|c|}{ Application rate, in tons per square mile } \\
\hline Monitoring-station name & $\begin{array}{c}\text { Monitoring- } \\
\text { station number }\end{array}$ & & Chloride & Sodium & Calcium \\
\hline \multirow[t]{3}{*}{ Great Egg Harbor near Sicklerville } & 01410784 & $\begin{array}{l}1975-76 \\
1976-77 \\
1977-78 \\
1978-79 \\
1979-80\end{array}$ & $\begin{array}{l}0.95 \\
1.18 \\
1.40 \\
1.47 \\
0.99\end{array}$ & $\begin{array}{l}0.56 \\
0.65 \\
0.75 \\
0.79 \\
0.54\end{array}$ & $\begin{array}{l}0.06 \\
0.13 \\
0.19 \\
0.20 \\
0.12\end{array}$ \\
\hline & & $\begin{array}{l}1980-81 \\
1981-82 \\
1982-83 \\
1983-84 \\
1984-85\end{array}$ & $\begin{array}{l}0.82 \\
1.44 \\
0.86 \\
1.51 \\
1.81\end{array}$ & $\begin{array}{l}0.46 \\
0.84 \\
0.48 \\
0.92 \\
1.14\end{array}$ & $\begin{array}{l}0.09 \\
0.10 \\
0.10 \\
0.06 \\
0.03\end{array}$ \\
\hline & & $\begin{array}{l}1985-86 \\
1986-87\end{array}$ & $\begin{array}{l}2.54 \\
3.14\end{array}$ & $\begin{array}{l}1.59 \\
1.98\end{array}$ & $\begin{array}{l}0.04 \\
0.04\end{array}$ \\
\hline \multirow[t]{3}{*}{ Great Egg Harbor near Blue Anchor } & 01410820 & $\begin{array}{l}1975-76 \\
1976-77 \\
1977-78 \\
1978-79 \\
1979-80\end{array}$ & $\begin{array}{l}0.95 \\
1.18 \\
1.40 \\
1.47 \\
0.99\end{array}$ & $\begin{array}{l}0.56 \\
0.65 \\
0.75 \\
0.79 \\
0.54\end{array}$ & $\begin{array}{l}0.06 \\
0.13 \\
0.19 \\
0.20 \\
0.12\end{array}$ \\
\hline & & $\begin{array}{l}1980-81 \\
1981-82 \\
1982-83 \\
1983-84 \\
1984-85\end{array}$ & $\begin{array}{l}0.82 \\
1.44 \\
0.86 \\
1.51 \\
1.81\end{array}$ & $\begin{array}{l}0.46 \\
0.84 \\
0.48 \\
0.92 \\
1.14\end{array}$ & $\begin{array}{l}0.09 \\
0.10 \\
0.10 \\
0.06 \\
0.03\end{array}$ \\
\hline & & $\begin{array}{l}1985-86 \\
1986-87\end{array}$ & $\begin{array}{l}2.54 \\
3.14\end{array}$ & $\begin{array}{l}1.59 \\
1.98\end{array}$ & $\begin{array}{l}0.04 \\
0.04\end{array}$ \\
\hline \multirow[t]{3}{*}{ Great Egg Harbor at Weymouth } & 01411110 & $\begin{array}{l}1975-76 \\
1976-77 \\
1977-78 \\
1978-79 \\
1979-80\end{array}$ & $\begin{array}{l}0.95 \\
1.18 \\
1.40 \\
1.47 \\
0.99\end{array}$ & $\begin{array}{l}0.56 \\
0.65 \\
0.75 \\
0.79 \\
0.54\end{array}$ & $\begin{array}{l}0.06 \\
0.13 \\
0.19 \\
0.20 \\
0.12\end{array}$ \\
\hline & & $\begin{array}{l}1980-81 \\
1981-82 \\
1982-83 \\
1983-84 \\
1984-85\end{array}$ & $\begin{array}{l}0.82 \\
1.44 \\
0.86 \\
1.51 \\
1.81\end{array}$ & $\begin{array}{l}0.46 \\
0.84 \\
0.48 \\
0.92 \\
1.14\end{array}$ & $\begin{array}{l}0.09 \\
0.10 \\
0.10 \\
0.06 \\
0.03\end{array}$ \\
\hline & & $\begin{array}{l}1985-86 \\
1986-87\end{array}$ & $\begin{array}{l}2.54 \\
3.14\end{array}$ & $\begin{array}{l}1.59 \\
1.98\end{array}$ & $\begin{array}{l}0.04 \\
0.04\end{array}$ \\
\hline \multirow[t]{3}{*}{ Maurice River at Norma } & 01411500 & $\begin{array}{l}1975-76 \\
1976-77 \\
1977-78 \\
1978-79 \\
1979-80\end{array}$ & $\begin{array}{l}0.95 \\
1.18 \\
1.40 \\
1.47 \\
0.99\end{array}$ & $\begin{array}{l}0.56 \\
0.65 \\
0.75 \\
0.79 \\
0.54\end{array}$ & $\begin{array}{l}0.06 \\
0.13 \\
0.19 \\
0.20 \\
0.12\end{array}$ \\
\hline & & $\begin{array}{l}1980-81 \\
1981-82 \\
1982-83 \\
1983-84 \\
1984-85\end{array}$ & $\begin{array}{l}0.82 \\
1.44 \\
0.86 \\
1.51 \\
1.81\end{array}$ & $\begin{array}{l}0.46 \\
0.84 \\
0.48 \\
0.92 \\
1.14\end{array}$ & $\begin{array}{l}0.09 \\
0.10 \\
0.10 \\
0.06 \\
0.03\end{array}$ \\
\hline & & $\begin{array}{l}1985-86 \\
1986-87\end{array}$ & $\begin{array}{l}2.54 \\
3.14\end{array}$ & $\begin{array}{l}1.59 \\
1.98\end{array}$ & $\begin{array}{l}0.04 \\
0.04\end{array}$ \\
\hline
\end{tabular}


Table I4. Estimated road-salt application in the New Jersey drainage basins studied, in terms of chloride, sodium, and calcium by basin and winter season, 1975-76 through 1986-87--Continued

[Source: A.T. Woodrow, New Jersey Department of Transportation, written commun., 1989]

\begin{tabular}{|c|c|c|c|c|c|}
\hline \multicolumn{2}{|l|}{ Drainage basin } & \multirow[b]{2}{*}{$\begin{array}{l}\text { Winter } \\
\text { season }\end{array}$} & \multicolumn{3}{|c|}{ Application rate, in tons per square mile } \\
\hline Monitoring-station name & $\begin{array}{l}\text { Monitoring- } \\
\text { station number }\end{array}$ & & Chloride & Sodium & Calcium \\
\hline \multirow[t]{12}{*}{ Cohansey River at Seeley } & 01412800 & $1975-76$ & 0.95 & 0.56 & 0.06 \\
\hline & & $1976-77$ & 1.18 & 0.65 & 0.13 \\
\hline & & $1977-78$ & 1.40 & 0.75 & 0.19 \\
\hline & & $1978-79$ & 1.47 & 0.79 & 0.20 \\
\hline & & $1979-80$ & 0.99 & 0.54 & 0.12 \\
\hline & & $1980-81$ & 0.82 & 0.46 & 0.09 \\
\hline & & $1981-82$ & 1.44 & 0.84 & 0.10 \\
\hline & & $1982-83$ & 0.86 & 0.48 & 0.10 \\
\hline & & $1983-84$ & 1.51 & 0.92 & 0.06 \\
\hline & & $1984-85$ & 1.81 & 1.14 & 0.03 \\
\hline & & $1985-86$ & 2.54 & 1.59 & 0.04 \\
\hline & & $1986-87$ & 3.14 & 1.98 & 0.04 \\
\hline \multirow[t]{12}{*}{ Paulins Kill at Blairstown } & 01443500 & $1975-76$ & 4.93 & 2.64 & 0.68 \\
\hline & & $1976-77$ & 4.29 & 2.26 & 0.65 \\
\hline & & $1977-78$ & 7.10 & 3.84 & 0.94 \\
\hline & & $\begin{array}{r}1978-79 \\
1079-80\end{array}$ & $\begin{array}{l}6.20 \\
3.23\end{array}$ & $\begin{array}{l}3.36 \\
1.74\end{array}$ & $\begin{array}{l}0.80 \\
0.44\end{array}$ \\
\hline & & $1919=80$ & & & \\
\hline & & $1980-81$ & 4.91 & 2.67 & 0.62 \\
\hline & & $1981-82$ & 9.53 & 5.82 & 0.36 \\
\hline & & $1982-83$ & 5.36 & 3.38 & 0.07 \\
\hline & & $1983-84$ & 10.38 & 6.43 & 0.27 \\
\hline & & $1984-85$ & 8.54 & 5.36 & 0.13 \\
\hline & & $1985-86$ & 10.16 & 6.40 & 0.13 \\
\hline & & $1986-87$ & 11.27 & 7.13 & 0.11 \\
\hline \multirow[t]{12}{*}{ Musconetcong River Outlet of Lake Hopatcong } & 01455500 & $1975-76$ & 4.93 & 2.64 & 0.68 \\
\hline & & $1976-77$ & 4.29 & 2.26 & 0.65 \\
\hline & & $1977-78$ & 7.10 & 3.84 & 0.94 \\
\hline & & $1978-79$ & 6.20 & 3.36 & 0.80 \\
\hline & & $1979-80$ & 3.23 & 1.74 & 0.44 \\
\hline & & $1980-81$ & 4.91 & 2.67 & 0.62 \\
\hline & & $1981-82$ & 9.53 & 5.82 & 0.36 \\
\hline & & $1982-83$ & 5.36 & 3.38 & 0.07 \\
\hline & & $1983-84$ & 10.38 & 6.43 & 0.27 \\
\hline & & $1984-85$ & 8.54 & 5.36 & 0.13 \\
\hline & & $1985-86$ & 10.16 & 6.40 & 0.13 \\
\hline & & $1986-87$ & 11.27 & 7.13 & 0.11 \\
\hline \multirow[t]{11}{*}{ Musconetcong River at Beattystown } & 01456200 & $1975-76$ & 4.93 & 2.64 & 0.68 \\
\hline & & $1976-77$ & 4.29 & 2.26 & 0.65 \\
\hline & & $1977-78$ & 7.10 & 3.84 & 0.94 \\
\hline & & $1978-79$ & 6.20 & 3.36 & 0.80 \\
\hline & & $1979-80$ & 3.23 & 1.74 & 0.44 \\
\hline & & $1980-81$ & 4.91 & 2.67 & 0.62 \\
\hline & & $1981-82$ & 9.53 & 5.82 & 0.36 \\
\hline & & $1982-83$ & 5.36 & 3.38 & 0.07 \\
\hline & & $1983-84$ & 10.38 & 6.43 & 0.27 \\
\hline & & $1984-85$ & 8.54 & 5.36 & 0.13 \\
\hline & & $\begin{array}{l}1985-86 \\
1986-87\end{array}$ & $\begin{array}{l}10.16 \\
11.27\end{array}$ & $\begin{array}{l}6.40 \\
7.13\end{array}$ & $\begin{array}{l}0.13 \\
0.11\end{array}$ \\
\hline
\end{tabular}


Table 14. Estimated road-salt application in the New Jersey drainage basins studied, in terms of chloride, sodium, and calcium by basin and winter season, 1975-76 through 1986-87--Continued

[Source: A.T. Woodrow, New Jersey Department of Transportation, written commun., 1989]

\begin{tabular}{|c|c|c|c|c|c|}
\hline \multicolumn{2}{|c|}{ Drainage basin } & \multirow[b]{2}{*}{$\begin{array}{l}\text { Winter } \\
\text { season }\end{array}$} & \multicolumn{3}{|c|}{ Application rate, in tons per square mile } \\
\hline Monitoring-station name & $\begin{array}{l}\text { Monitoring- } \\
\text { station number }\end{array}$ & & Chloride & Sodium & Calcium \\
\hline \multirow[t]{12}{*}{ Musconetcong River at Riegelsville } & 01457400 & $1975-76$ & 4.93 & 2.64 & 0.68 \\
\hline & & $1976-77$ & 4.29 & 2.26 & 0.65 \\
\hline & & $1977-78$ & 7.10 & 3.84 & 0.94 \\
\hline & & $1978-79$ & 6.20 & 3.36 & 0.80 \\
\hline & & $1979-80$ & 3.23 & 1.74 & 0.44 \\
\hline & & $1980-81$ & 4.91 & 2.67 & 0.62 \\
\hline & & $1981-82$ & 9.53 & 5.82 & 0.36 \\
\hline & & $1982-83$ & 5.36 & 3.38 & 0.07 \\
\hline & & $1983-84$ & 10.38 & 6.43 & 0.27 \\
\hline & & $1984-85$ & 8.54 & 5.36 & 0.13 \\
\hline & & $1985-86$ & 10.16 & 6.40 & 0.13 \\
\hline & & 1986-87 & 11.27 & 7.13 & 0.11 \\
\hline \multirow[t]{12}{*}{ Wickechcoke Creek at Stockton } & 01461300 & $1975-76$ & 4.93 & 2.64 & 0.68 \\
\hline & & $1976-77$ & 4.29 & 2.26 & 0.65 \\
\hline & & $1977-78$ & 7.10 & 3.84 & 0.94 \\
\hline & & 1978-79 & 6.20 & 3.36 & 0.80 \\
\hline & & $1979-80$ & 3.23 & 1.74 & 0.44 \\
\hline & & $1980-81$ & 4.91 & 2.67 & 0.62 \\
\hline & & $1981-82$ & 9.53 & 5.82 & 0.36 \\
\hline & & $1982-83$ & 5.36 & 3.38 & 0.07 \\
\hline & & 1983-84 & 10.38 & 6.43 & 0.27 \\
\hline & & $1984-85$ & 8.54 & 5.36 & 0.13 \\
\hline & & $1985-86$ & 10.16 & 6.40 & 0.13 \\
\hline & & $1986-87$ & 11.27 & 7.13 & 0.11 \\
\hline \multirow[t]{12}{*}{ Crosswicks Creek at Extonville } & 01464500 & $1975-76$ & 3.20 & 1.91 & 0.19 \\
\hline & & $1976-77$ & 2.94 & 1.66 & 0.29 \\
\hline & & $1977-78$ & 3.60 & 1.99 & 0.42 \\
\hline & & $1978-79$ & 3.13 & 1.70 & 0.40 \\
\hline & & 1979-80 & 1.84 & 1.02 & 0.22 \\
\hline & & $1980-81$ & 2.28 & 1.27 & 0.26 \\
\hline & & $1981-82$ & 3.92 & 2.34 & 0.23 \\
\hline & & $1982-83$ & 2.05 & 1.26 & 0.06 \\
\hline & & $1983-84$ & 3.87 & 2.43 & 0.05 \\
\hline & & $1984-85$ & 3.24 & 2.00 & 0.10 \\
\hline & & $1985-86$ & 3.91 & 2.47 & 0.05 \\
\hline & & $1986-87$ & 6.09 & 3.85 & 0.06 \\
\hline \multirow[t]{12}{*}{ Doctors Creek at Allentown } & 01464515 & $1975-76$ & 4.23 & 2.52 & 0.25 \\
\hline & & $1976-77$ & 3.73 & 2.12 & 0.36 \\
\hline & & $1977-78$ & 4.60 & 2.55 & 0.52 \\
\hline & & $1978-79$ & 3.88 & 2.11 & 0.49 \\
\hline & & 1979-80 & 2.23 & 1.23 & 0.26 \\
\hline & & $1980-81$ & 2.94 & 1.63 & 0.33 \\
\hline & & $1981-82$ & 5.04 & 3.01 & 0.28 \\
\hline & & 1982-83 & 2.58 & 1.62 & 0.05 \\
\hline & & $1983-84$ & 4.93 & 3.12 & 0.05 \\
\hline & & $1984-85$ & 3.89 & 2.39 & 0.13 \\
\hline & & $1985-86$ & 4.53 & 2.86 & 0.05 \\
\hline & & $1986-87$ & 7.43 & 4.70 & 0.07 \\
\hline
\end{tabular}


Table 14. Estimated road-salt application in the New Jersey drainage basins studied, in terms of chloride, sodium, and calcium by basin and winter season, 1975-76 through 1986-87--Continued

[Source: A.T. Woodrow, New Jersey Department of Transportation, written commun., 1989]

\begin{tabular}{|c|c|c|c|c|c|}
\hline \multicolumn{2}{|l|}{ Drainage basin } & \multirow[b]{2}{*}{$\begin{array}{l}\text { Winter } \\
\text { season }\end{array}$} & \multicolumn{3}{|c|}{ Application rate, in tons per square mile } \\
\hline Monitoring-station name & $\begin{array}{l}\text { Monitoring- } \\
\text { station number }\end{array}$ & & Chloride & Sodium & Calcium \\
\hline \multirow[t]{12}{*}{ SB Rancocas Creek at Vincentown } & 01465850 & $1975-76$ & 0.95 & 0.56 & 0.06 \\
\hline & & $1976-77$ & 1.18 & 0.65 & 0.13 \\
\hline & & $1977-78$ & 1.40 & 0.75 & 0.19 \\
\hline & & $1978-79$ & 1.47 & 0.79 & 0.20 \\
\hline & & $1979-80$ & 0.99 & 0.54 & 0.12 \\
\hline & & $1980-81$ & 0.82 & 0.46 & 0.09 \\
\hline & & $1981-82$ & 1.44 & 0.84 & 0.10 \\
\hline & & $1982-83$ & 0.86 & 0.48 & 0.10 \\
\hline & & $1983-84$ & 1.51 & 0.92 & 0.06 \\
\hline & & $1984-85$ & 1.81 & 1.14 & 0.03 \\
\hline & & $1985-86$ & 2.54 & 1.59 & 0.04 \\
\hline & & $1986-87$ & 3.14 & 1.98 & 0.04 \\
\hline \multirow[t]{12}{*}{ NB Rancocas Creek at Browns Mills } & 01465970 & $1975-76$ & 2.25 & 1.34 & 0.13 \\
\hline & & $1976-77$ & 2.19 & 1.23 & 0.23 \\
\hline & & $1977-78$ & 2.67 & 1.47 & 0.32 \\
\hline & & $1978-79$ & 2.43 & 1.32 & 0.32 \\
\hline & & $1979-80$ & 1.48 & 0.81 & 0.18 \\
\hline & & $1980-81$ & 1.67 & 0.93 & 0.18 \\
\hline & & $1981-82$ & 2.87 & 1.71 & 0.17 \\
\hline & & $1982-83$ & 1.55 & 0.93 & 0.08 \\
\hline & & 1983-84 & 2.87 & 1.79 & 0.05 \\
\hline & & $1984-85$ & 2.64 & 1.64 & 0.07 \\
\hline & & $1985-86$ & 3.33 & 2.10 & 0.04 \\
\hline & & $1986-87$ & 4.84 & 3.06 & 0.05 \\
\hline \multirow[t]{12}{*}{ McDonalds Branch in Lebanon State Forest } & 01466500 & $1975-76$ & 0.95 & 0.56 & 0.06 \\
\hline & & $1976-77$ & 1.18 & 0.65 & 0.13 \\
\hline & & $1977-78$ & 1.40 & 0.75 & 0.19 \\
\hline & & $1978-79$ & 1.47 & 0.79 & 0.20 \\
\hline & & $1979-80$ & 0.99 & 0.54 & 0.12 \\
\hline & & $1980-81$ & 0.82 & 0.46 & 0.09 \\
\hline & & $1981-82$ & 1.44 & 0.84 & 0.10 \\
\hline & & $1982-83$ & 0.86 & 0.48 & 0.10 \\
\hline & & $1983-84$ & 1.51 & 0.92 & 0.06 \\
\hline & & $1984-85$ & 1.81 & 1.14 & 0.03 \\
\hline & & $1985-86$ & 2.54 & 1.59 & 0.04 \\
\hline & & $1986-87$ & 3.14 & 1.98 & 0.04 \\
\hline \multirow[t]{12}{*}{ NB Rancocas Creek at Browns Mills } & 01467000 & $1975-76$ & 2.14 & 1.28 & 0.13 \\
\hline & & $1976-77$ & 2.11 & 1.19 & 0.22 \\
\hline & & $1977-78$ & 2.56 & 1.41 & 0.31 \\
\hline & & $1978-79$ & 2.35 & 1.27 & 0.31 \\
\hline & & $1979-80$ & 1.44 & 0.79 & 0.17 \\
\hline & & $1980-81$ & 1.60 & 0.89 & 0.18 \\
\hline & & $1981-82$ & 2.75 & 1.63 & 0.17 \\
\hline & & $1982-83$ & 1.49 & 0.89 & 0.08 \\
\hline & & $1983-84$ & 2.76 & 1.72 & 0.05 \\
\hline & & $1984-85$ & 2.57 & 1.60 & 0.06 \\
\hline & & $\begin{array}{l}1985-86 \\
1986-87\end{array}$ & $\begin{array}{l}3.26 \\
470\end{array}$ & 2.05 & 0.04 \\
\hline & & $1986-87$ & 4.70 & 2.97 & 0.05 \\
\hline
\end{tabular}


Table 14. Estimated road-salt application in the New Jersey drainage basins studied, in terms of chloride, sodium, and calcium by basin and winter season, 1975-76 through 1986-87--Continued

[Source: A.T. Woodrow, New Jersey Department of Transportation, written commun., 1989]

\begin{tabular}{|c|c|c|c|c|c|}
\hline \multicolumn{2}{|l|}{ Drainage basin } & \multirow[b]{2}{*}{$\begin{array}{l}\text { Winter } \\
\text { season }\end{array}$} & \multicolumn{3}{|c|}{ Application rate, in tons per square mile } \\
\hline Monitoring-station name & $\begin{array}{c}\text { Monitoring- } \\
\text { station number }\end{array}$ & & Chloride & Sodium & Calcium \\
\hline \multirow[t]{3}{*}{ NB Pennsauken Creek near Moorestown } & 01467069 & $\begin{array}{l}1975-76 \\
1976-77 \\
1977-78 \\
1978-79 \\
1979-80\end{array}$ & $\begin{array}{l}0.95 \\
1.18 \\
1.40 \\
1.47 \\
0.99\end{array}$ & $\begin{array}{l}0.56 \\
0.65 \\
0.75 \\
0.79 \\
0.54\end{array}$ & $\begin{array}{l}0.06 \\
0.13 \\
0.19 \\
0.20 \\
0.12\end{array}$ \\
\hline & & $\begin{array}{l}1980-81 \\
1981-82 \\
1982-83 \\
1983-84 \\
1984-85\end{array}$ & $\begin{array}{l}0.82 \\
1.44 \\
0.86 \\
1.51 \\
1.81\end{array}$ & $\begin{array}{l}0.46 \\
0.84 \\
0.48 \\
0.92 \\
1.14\end{array}$ & $\begin{array}{l}0.09 \\
0.10 \\
0.10 \\
0.06 \\
0.03\end{array}$ \\
\hline & & $\begin{array}{l}1985-86 \\
1986-87\end{array}$ & $\begin{array}{l}2.54 \\
3.14\end{array}$ & $\begin{array}{l}1.59 \\
1.98\end{array}$ & $\begin{array}{l}0.04 \\
0.04\end{array}$ \\
\hline \multirow[t]{3}{*}{ SB Pennsauken Creek at Cherry Hill } & 01467081 & $\begin{array}{l}1975-76 \\
1976-77 \\
1977-78 \\
1978-79 \\
1979-80\end{array}$ & $\begin{array}{l}0.95 \\
1.18 \\
1.40 \\
1.47 \\
0.99\end{array}$ & $\begin{array}{l}0.56 \\
0.65 \\
0.75 \\
0.79 \\
0.54\end{array}$ & $\begin{array}{l}0.06 \\
0.13 \\
0.19 \\
0.20 \\
0.12\end{array}$ \\
\hline & & $\begin{array}{l}1980-81 \\
1981-82 \\
1982-83 \\
1983-84 \\
1984-85\end{array}$ & $\begin{array}{l}0.82 \\
1.44 \\
0.86 \\
1.51 \\
1.81\end{array}$ & $\begin{array}{l}0.46 \\
0.84 \\
0.48 \\
0.92 \\
1.14\end{array}$ & $\begin{array}{l}0.09 \\
0.10 \\
0.10 \\
0.06 \\
0.03\end{array}$ \\
\hline & & $\begin{array}{l}1985-86 \\
1986-87\end{array}$ & $\begin{array}{l}2.54 \\
3.14\end{array}$ & $\begin{array}{l}1.59 \\
1.98\end{array}$ & $\begin{array}{l}0.04 \\
0.04\end{array}$ \\
\hline \multirow[t]{3}{*}{ Cooper River at Norcross Rd at Lindenwold } & 01467120 & $\begin{array}{l}1975-76 \\
1976-77 \\
1977-78 \\
1978-79 \\
1979-80\end{array}$ & $\begin{array}{l}0.95 \\
1.18 \\
1.40 \\
1.47 \\
0.99\end{array}$ & $\begin{array}{l}0.56 \\
0.65 \\
0.75 \\
0.79 \\
0.54\end{array}$ & $\begin{array}{l}0.06 \\
0.13 \\
0.19 \\
0.20 \\
0.12\end{array}$ \\
\hline & & $\begin{array}{l}1980-81 \\
1981-82 \\
1982-83 \\
1983-84 \\
1984-85\end{array}$ & $\begin{array}{l}0.82 \\
1.44 \\
0.86 \\
1.51 \\
1.81\end{array}$ & $\begin{array}{l}0.46 \\
0.84 \\
0.48 \\
0.92 \\
1.14\end{array}$ & $\begin{array}{l}0.09 \\
0.10 \\
0.10 \\
0.06 \\
0.03\end{array}$ \\
\hline & & $\begin{array}{l}1985-86 \\
1986-87\end{array}$ & $\begin{array}{l}2.54 \\
3.14\end{array}$ & $\begin{array}{l}1.59 \\
1.98\end{array}$ & $\begin{array}{l}0.04 \\
0.04\end{array}$ \\
\hline \multirow[t]{3}{*}{ Cooper River at Lawnside } & 01467140 & $\begin{array}{l}1975-76 \\
1976-77 \\
1977-78 \\
1978-79 \\
1979-80\end{array}$ & $\begin{array}{l}0.95 \\
1.18 \\
1.40 \\
1.47 \\
0.99\end{array}$ & $\begin{array}{l}0.56 \\
0.65 \\
0.75 \\
0.79 \\
0.54\end{array}$ & $\begin{array}{l}0.06 \\
0.13 \\
0.19 \\
0.20 \\
0.12\end{array}$ \\
\hline & & $\begin{array}{l}1980-81 \\
1981-82 \\
1982-83 \\
1983-84 \\
1984-85\end{array}$ & $\begin{array}{l}0.82 \\
1.44 \\
0.86 \\
1.51 \\
1.81\end{array}$ & $\begin{array}{l}0.46 \\
0.84 \\
0.48 \\
0.92 \\
1.14\end{array}$ & $\begin{array}{l}0.09 \\
0.10 \\
0.10 \\
0.06 \\
0.03\end{array}$ \\
\hline & & $\begin{array}{l}1985-86 \\
1986-87\end{array}$ & $\begin{array}{l}2.54 \\
3.14\end{array}$ & $\begin{array}{l}1.59 \\
1.98\end{array}$ & $\begin{array}{l}0.04 \\
0.04\end{array}$ \\
\hline
\end{tabular}


Table I4. Estimated road-salt application in the New Jersey drainage basins studied, in terms of chloride, sodium, and calcium by basin and winter season, 1975-76 through 1986-87--Continued

[Source: A.T. Woodrow, New Jersey Department of Transportation, written commun., 1989]

\begin{tabular}{|c|c|c|c|c|c|}
\hline \multicolumn{2}{|l|}{ Drainage basin } & \multirow[b]{2}{*}{$\begin{array}{l}\text { Winter } \\
\text { season }\end{array}$} & \multicolumn{3}{|c|}{ Application rate, in tons per square mile } \\
\hline Monitoring-station name & $\begin{array}{c}\text { Monitoring- } \\
\text { station number }\end{array}$ & & Chloride & Sodium & Calcium \\
\hline \multirow[t]{3}{*}{ SB Big Timber Creek at Blackwood Terrace } & 01467329 & $\begin{array}{l}1975-76 \\
1976-77 \\
1977-78 \\
1978-79 \\
1979-80\end{array}$ & $\begin{array}{l}0.95 \\
1.18 \\
1.40 \\
1.47 \\
0.99\end{array}$ & $\begin{array}{l}0.56 \\
0.65 \\
0.75 \\
0.79 \\
0.54\end{array}$ & $\begin{array}{l}0.06 \\
0.13 \\
0.19 \\
0.20 \\
0.12\end{array}$ \\
\hline & & $\begin{array}{l}1980-81 \\
1981-82 \\
1982-83 \\
1983-84 \\
1984-85\end{array}$ & $\begin{array}{l}0.82 \\
1.44 \\
0.86 \\
1.51 \\
1.81\end{array}$ & $\begin{array}{l}0.46 \\
0.84 \\
0.48 \\
0.92 \\
1.14\end{array}$ & $\begin{array}{l}0.09 \\
0.10 \\
0.10 \\
0.06 \\
0.03\end{array}$ \\
\hline & & $\begin{array}{l}1985-86 \\
1986-87\end{array}$ & $\begin{array}{l}2.54 \\
3.14\end{array}$ & $\begin{array}{l}1.59 \\
1.98\end{array}$ & $\begin{array}{l}0.04 \\
0.04\end{array}$ \\
\hline \multirow[t]{3}{*}{ Raccoon Creek near Swedesboro } & 01477120 & $\begin{array}{l}1975-76 \\
1976-77 \\
1977-78 \\
1978-79 \\
1979-80\end{array}$ & $\begin{array}{l}0.95 \\
1.18 \\
1.40 \\
1.47 \\
0.99\end{array}$ & $\begin{array}{l}0.56 \\
0.65 \\
0.75 \\
0.79 \\
0.54\end{array}$ & $\begin{array}{l}0.06 \\
0.13 \\
0.19 \\
0.20 \\
0.12\end{array}$ \\
\hline & & $\begin{array}{l}1980-81 \\
1981-82 \\
1982-83 \\
1983-84 \\
1984-85\end{array}$ & $\begin{array}{l}0.82 \\
1.44 \\
0.86 \\
1.51 \\
1.81\end{array}$ & $\begin{array}{l}0.46 \\
0.84 \\
0.48 \\
0.92 \\
1.14\end{array}$ & $\begin{array}{l}0.09 \\
0.10 \\
0.10 \\
0.06 \\
0.03\end{array}$ \\
\hline & & $\begin{array}{l}1985-86 \\
1986-87\end{array}$ & $\begin{array}{l}2.54 \\
3.14\end{array}$ & $\begin{array}{l}1.59 \\
1.98\end{array}$ & $\begin{array}{l}0.04 \\
0.04\end{array}$ \\
\hline \multirow[t]{3}{*}{ Oldmans Creek at Porches Mill } & 01477510 & $\begin{array}{l}1975-76 \\
1976-77 \\
1977-78 \\
1978-79 \\
1979-80\end{array}$ & $\begin{array}{l}0.95 \\
1.18 \\
1.40 \\
1.47 \\
0.99\end{array}$ & $\begin{array}{l}0.56 \\
0.65 \\
0.75 \\
0.79 \\
0.54\end{array}$ & $\begin{array}{l}0.06 \\
0.13 \\
0.19 \\
0.20 \\
0.12\end{array}$ \\
\hline & & $\begin{array}{l}1980-81 \\
1981-82 \\
1982-83 \\
1983-84 \\
1984-85\end{array}$ & $\begin{array}{l}0.82 \\
1.44 \\
0.86 \\
1.51 \\
1.81\end{array}$ & $\begin{array}{l}0.46 \\
0.84 \\
0.48 \\
0.92 \\
1.14\end{array}$ & $\begin{array}{l}0.09 \\
0.10 \\
0.10 \\
0.06 \\
0.03\end{array}$ \\
\hline & & $\begin{array}{l}1985-86 \\
1986-87\end{array}$ & $\begin{array}{l}2.54 \\
3.14\end{array}$ & $\begin{array}{l}1.59 \\
1.98\end{array}$ & $\begin{array}{l}0.04 \\
0.04\end{array}$ \\
\hline \multirow[t]{3}{*}{ Salem River at Woodstown } & 01482500 & $\begin{array}{l}1975-76 \\
1976-77 \\
1977-78 \\
1978-79 \\
1979-80\end{array}$ & $\begin{array}{l}0.95 \\
1.18 \\
1.40 \\
1.47 \\
0.99\end{array}$ & $\begin{array}{l}0.56 \\
0.65 \\
0.75 \\
0.79 \\
0.54\end{array}$ & $\begin{array}{l}0.06 \\
0.13 \\
0.19 \\
0.20 \\
0.12\end{array}$ \\
\hline & & $\begin{array}{c}1980-81 \\
1981-82 \\
1982-83 \\
1983-84 \\
1984-85\end{array}$ & $\begin{array}{l}0.82 \\
1.44 \\
0.86 \\
1.51 \\
1.81\end{array}$ & $\begin{array}{l}0.46 \\
0.84 \\
0.48 \\
0.92 \\
1.14\end{array}$ & $\begin{array}{l}0.09 \\
0.10 \\
0.10 \\
0.06 \\
0.03\end{array}$ \\
\hline & & $\begin{array}{l}1985-86 \\
1986-87 \\
\end{array}$ & $\begin{array}{l}2.54 \\
3.14 \\
\end{array}$ & $\begin{array}{l}1.59 \\
1.98 \\
\end{array}$ & $\begin{array}{l}0.04 \\
0.04 \\
\end{array}$ \\
\hline
\end{tabular}


Table 16. Estimated application of phosphorus and nitrogen fertilizer in the New Jersey drainage basins studied, 1975-85

[Based on data supplied by R.B. Alexander, U.S. Geological Survey, written commun., 1989]

\begin{tabular}{|c|c|c|c|c|}
\hline \multicolumn{2}{|c|}{ Drainage basin } & \multirow[b]{2}{*}{ Year } & \multicolumn{2}{|c|}{$\begin{array}{l}\text { Fertilizer application rate, in tons per } \\
\text { square mile }\end{array}$} \\
\hline Monitoring-station name & $\begin{array}{l}\text { Monitoring- } \\
\text { station number }\end{array}$ & & Phosphorus & Nitrogen \\
\hline \multirow[t]{11}{*}{ Wallkill River at Franklin } & 01367700 & 1975 & 1.42 & 1.75 \\
\hline & & 1976 & 1.40 & 1.80 \\
\hline & & 1977 & 1.53 & 1.75 \\
\hline & & 1978 & 1.25 & 1.65 \\
\hline & & 1979 & 1.34 & 1.78 \\
\hline & & 1980 & 1.32 & 1.78 \\
\hline & & 1981 & 1.27 & 1.90 \\
\hline & & 1982 & 1.29 & 1.91 \\
\hline & & 1983 & 1.07 & 1.49 \\
\hline & & 1984 & 1.34 & 1.95 \\
\hline & & 1985 & 1.29 & 2.08 \\
\hline \multirow[t]{11}{*}{ Wallkill River near Sussex } & 01367770 & 1975 & 1.41 & 1.73 \\
\hline & & 1976 & 1.38 & 1.78 \\
\hline & & 1977 & 1.52 & 1.73 \\
\hline & & 1978 & 1.23 & 1.64 \\
\hline & & 1979 & 1.33 & 1.76 \\
\hline & & 1980 & 1.31 & 1.76 \\
\hline & & 1981 & 1.26 & 1.88 \\
\hline & & 1982 & 1.27 & 1.89 \\
\hline & & 1983 & 1.06 & 1.48 \\
\hline & & 1984 & 1.33 & 1.93 \\
\hline & & 1985 & 1.28 & 2.06 \\
\hline \multirow[t]{11}{*}{ Papakating Creek at Sussex } & 01367910 & 1975 & 1.40 & 1.73 \\
\hline & & 1976 & 1.38 & 1.78 \\
\hline & & 1977 & 1.51 & 1.73 \\
\hline & & 1978 & 1.23 & 1.63 \\
\hline & & 1979 & 1.32 & 1.76 \\
\hline & & 1980 & 1.30 & 1.75 \\
\hline & & 1981 & 1.25 & 1.87 \\
\hline & & 1982 & 1.27 & 1.88 \\
\hline & & 1983 & 1.05 & 1.47 \\
\hline & & 1984 & 1.32 & 1.93 \\
\hline & & 1985 & 1.27 & 2.05 \\
\hline \multirow[t]{11}{*}{ Black Creek near Vernon } & 01368950 & 1975 & 1.40 & 1.73 \\
\hline & & 1976 & 1.38 & 1.78 \\
\hline & & 1977 & 1.51 & 1.73 \\
\hline & & 1978 & 1.23 & 1.63 \\
\hline & & 1979 & 1.32 & 1.76 \\
\hline & & 1980 & 1.30 & 1.75 \\
\hline & & 1981 & 1.25 & 1.87 \\
\hline & & 1982 & 1.27 & 1.88 \\
\hline & & 1983 & 1.05 & 1.47 \\
\hline & & 1984 & 1.32 & 1.93 \\
\hline & & 1985 & 1.27 & 2.05 \\
\hline
\end{tabular}


Table 16. Estimated application of phosphorus and nitrogen fertilizer in the New Jersey drainage basins studied, 1975-85--Continued

[Based on data supplied by R.B. Alexander, U.S. Geological Survey, written commun.,1989]

\begin{tabular}{|c|c|c|c|c|}
\hline \multicolumn{2}{|l|}{ Drainage basin } & \multirow[b]{2}{*}{ Year } & \multicolumn{2}{|c|}{$\begin{array}{l}\text { Fertilizer application rate, in tons per } \\
\text { square mile }\end{array}$} \\
\hline Monitoring-station name & $\begin{array}{l}\text { Monitoring- } \\
\text { station number }\end{array}$ & & Phosphorus & Nitrogen \\
\hline Passaic River near Millington & 01379000 & $\begin{array}{l}1975 \\
1976 \\
1977 \\
1978 \\
1979\end{array}$ & $\begin{array}{l}1.13 \\
1.12 \\
1.22 \\
0.99 \\
1.07\end{array}$ & $\begin{array}{l}1.40 \\
1.44 \\
1.40 \\
1.32 \\
1.42\end{array}$ \\
\hline & & $\begin{array}{l}1980 \\
1981 \\
1982 \\
1983 \\
1984\end{array}$ & $\begin{array}{l}1.05 \\
1.01 \\
1.02 \\
0.85 \\
1.07\end{array}$ & $\begin{array}{l}1.42 \\
1.51 \\
1.52 \\
1.19 \\
1.56\end{array}$ \\
\hline & & 1985 & 1.03 & 1.66 \\
\hline Passaic River near Chatham & 01379500 & $\begin{array}{l}1975 \\
1976 \\
1977 \\
1978 \\
1979\end{array}$ & $\begin{array}{l}1.59 \\
1.56 \\
1.71 \\
1.39 \\
1.50\end{array}$ & $\begin{array}{l}1.96 \\
2.01 \\
1.96 \\
1.85 \\
1.99\end{array}$ \\
\hline & & $\begin{array}{l}1980 \\
1981 \\
1982 \\
1983 \\
1984\end{array}$ & $\begin{array}{l}1.48 \\
1.42 \\
1.44 \\
1.19 \\
1.50\end{array}$ & $\begin{array}{l}1.99 \\
2.12 \\
2.13 \\
1.67 \\
2.18\end{array}$ \\
\hline & & 1985 & 1.44 & 2.32 \\
\hline Rockaway River at Pine Brook & 01381200 & $\begin{array}{l}1975 \\
1976 \\
1977 \\
1978 \\
1979\end{array}$ & $\begin{array}{l}0.75 \\
0.74 \\
0.81 \\
0.66 \\
0.71\end{array}$ & $\begin{array}{l}0.93 \\
0.96 \\
0.93 \\
0.88 \\
0.95\end{array}$ \\
\hline & & $\begin{array}{l}1980 \\
1981 \\
1982 \\
1983 \\
1984\end{array}$ & $\begin{array}{l}0.70 \\
0.67 \\
0.68 \\
0.57 \\
0.71\end{array}$ & $\begin{array}{l}0.94 \\
1.01 \\
1.01 \\
0.79 \\
1.04\end{array}$ \\
\hline & & 1985 & 0.69 & 1.10 \\
\hline Whippany River at Morristown & 01381500 & $\begin{array}{l}1975 \\
1976 \\
1977 \\
1978 \\
1979\end{array}$ & $\begin{array}{l}0.72 \\
0.71 \\
0.77 \\
0.63 \\
0.68\end{array}$ & $\begin{array}{l}0.89 \\
0.91 \\
0.88 \\
0.84 \\
0.90\end{array}$ \\
\hline & & $\begin{array}{l}1980 \\
1981 \\
1982 \\
1983 \\
1984\end{array}$ & $\begin{array}{l}0.67 \\
0.64 \\
0.65 \\
0.54 \\
0.68\end{array}$ & $\begin{array}{l}0.90 \\
0.96 \\
0.96 \\
0.75 \\
0.99\end{array}$ \\
\hline & & 1985 & 0.65 & 1.05 \\
\hline
\end{tabular}


Table 16. Estimated application of phosphorus and nitrogen fertilizer in the New Jersey drainage basins studied, 1975-85--Continued

[Based on data supplied by R.B. Alexander, U.S. Geological Survey, written commun., 1989]

\begin{tabular}{|c|c|c|c|c|}
\hline \multicolumn{2}{|c|}{ Drainage basin } & \multirow[b]{2}{*}{ Year } & \multicolumn{2}{|c|}{$\begin{array}{c}\text { Fertilizer application rate, in tons per } \\
\text { square mile }\end{array}$} \\
\hline Monitoring-station name & $\begin{array}{l}\text { Monitoring- } \\
\text { station number }\end{array}$ & & Phosphorus & Nitrogen \\
\hline \multirow[t]{3}{*}{ Whippany River near Pine Brook } & 01381800 & $\begin{array}{l}1975 \\
1976 \\
1977 \\
1978 \\
1979\end{array}$ & $\begin{array}{l}0.72 \\
0.71 \\
0.77 \\
0.63 \\
0.68\end{array}$ & $\begin{array}{l}0.89 \\
0.91 \\
0.88 \\
0.84 \\
0.90\end{array}$ \\
\hline & & $\begin{array}{l}1980 \\
1981 \\
1982 \\
1983 \\
1984\end{array}$ & $\begin{array}{l}0.67 \\
0.64 \\
0.65 \\
0.54 \\
0.68\end{array}$ & $\begin{array}{l}0.90 \\
0.96 \\
0.96 \\
0.75 \\
0.99\end{array}$ \\
\hline & & 1985 & 0.65 & 1.05 \\
\hline \multirow[t]{3}{*}{ Passaic River at Two Bridges } & 01382000 & $\begin{array}{l}1975 \\
1976 \\
1977 \\
1978 \\
1979\end{array}$ & $\begin{array}{l}0.90 \\
0.89 \\
0.97 \\
0.79 \\
0.85\end{array}$ & $\begin{array}{l}1.12 \\
1.14 \\
1.11 \\
1.05 \\
1.13\end{array}$ \\
\hline & & $\begin{array}{l}1980 \\
1981 \\
1982 \\
1983 \\
1984\end{array}$ & $\begin{array}{l}0.84 \\
0.81 \\
0.82 \\
0.68 \\
0.85\end{array}$ & $\begin{array}{l}1.13 \\
1.21 \\
1.21 \\
0.95 \\
1.24\end{array}$ \\
\hline & & 1985 & 0.82 & 1.32 \\
\hline \multirow[t]{3}{*}{ Wanaque River at Wanaque } & 01387000 & $\begin{array}{l}1975 \\
1976 \\
1977 \\
1978 \\
1979\end{array}$ & $\begin{array}{l}0.01 \\
0.01 \\
0.01 \\
0.01 \\
0.01\end{array}$ & $\begin{array}{l}0.02 \\
0.02 \\
0.02 \\
0.02 \\
0.02\end{array}$ \\
\hline & & $\begin{array}{l}1980 \\
1981 \\
1982 \\
1983 \\
1984\end{array}$ & $\begin{array}{l}0.01 \\
0.01 \\
0.01 \\
0.01 \\
0.01\end{array}$ & $\begin{array}{l}0.02 \\
0.02 \\
0.02 \\
0.01 \\
0.02\end{array}$ \\
\hline & & 1985 & 0.01 & 0.02 \\
\hline \multirow[t]{3}{*}{ Saddle River at Lodi } & 01391500 & $\begin{array}{l}1975 \\
1976 \\
1977 \\
1978 \\
1979\end{array}$ & $\begin{array}{l}0.14 \\
0.14 \\
0.15 \\
0.13 \\
0.13\end{array}$ & $\begin{array}{l}0.17 \\
0.18 \\
0.17 \\
0.17 \\
0.18\end{array}$ \\
\hline & & $\begin{array}{l}1980 \\
1981 \\
1982 \\
1983 \\
1984\end{array}$ & $\begin{array}{l}0.13 \\
0.13 \\
0.13 \\
0.11 \\
0.13\end{array}$ & $\begin{array}{l}0.18 \\
0.19 \\
0.19 \\
0.15 \\
0.19\end{array}$ \\
\hline & & 1985 & 0.13 & 0.21 \\
\hline
\end{tabular}


Table 16. Estimated application of phosphorus and nitrogen fertilizer in the New Jersey drainage basins studied, 1975-85--Continued

[Based on data supplied by R.B. Alexander, U.S. Geological Survey, written commun.,1989]

\begin{tabular}{|c|c|c|c|c|}
\hline \multicolumn{2}{|l|}{ Drainage basin } & \multirow[b]{2}{*}{ Year } & \multicolumn{2}{|c|}{$\begin{array}{l}\text { Fertilizer application rate, in tons per } \\
\text { square mile }\end{array}$} \\
\hline Monitoring-station name & $\begin{array}{l}\text { Monitoring- } \\
\text { station number }\end{array}$ & & Phosphorus & Nitrogen \\
\hline \multirow[t]{3}{*}{ SB Raritan River at Middle Valley } & 01396280 & $\begin{array}{l}1975 \\
1976 \\
1977 \\
1978 \\
1979\end{array}$ & $\begin{array}{l}0.72 \\
0.71 \\
0.77 \\
0.63 \\
0.68\end{array}$ & $\begin{array}{l}0.89 \\
0.91 \\
0.88 \\
0.84 \\
0.90\end{array}$ \\
\hline & & $\begin{array}{l}1980 \\
1981 \\
1982 \\
1983 \\
1984\end{array}$ & $\begin{array}{l}0.67 \\
0.64 \\
0.65 \\
0.54 \\
0.68\end{array}$ & $\begin{array}{l}0.90 \\
0.96 \\
0.96 \\
0.75 \\
0.99\end{array}$ \\
\hline & & 1985 & 0.65 & 1.05 \\
\hline \multirow[t]{3}{*}{ SB Raritan Raritan River at High Bridge } & 01396535 & $\begin{array}{l}1975 \\
1976 \\
1977 \\
1978 \\
1979\end{array}$ & $\begin{array}{l}1.63 \\
1.61 \\
1.76 \\
1.43 \\
1.54\end{array}$ & $\begin{array}{l}2.01 \\
2.07 \\
2.01 \\
1.90 \\
2.05\end{array}$ \\
\hline & & $\begin{array}{l}1980 \\
1981 \\
1982 \\
1983 \\
1984\end{array}$ & $\begin{array}{l}1.52 \\
1.46 \\
1.47 \\
1.23 \\
1.54\end{array}$ & $\begin{array}{l}2.04 \\
2.18 \\
2.19 \\
1.71 \\
2.24\end{array}$ \\
\hline & & 1985. & 1.48 & 2.39 \\
\hline \multirow[t]{3}{*}{ Mulhockaway Creek at Van Syckel } & 01396660 & $\begin{array}{l}1975 \\
1976 \\
1977 \\
1978 \\
1979\end{array}$ & $\begin{array}{l}4.85 \\
4.77 \\
5.23 \\
4.25 \\
4.58\end{array}$ & $\begin{array}{l}5.98 \\
6.14 \\
5.97 \\
5.64 \\
6.08\end{array}$ \\
\hline & & $\begin{array}{l}1980 \\
1981 \\
1982 \\
1983 \\
1984\end{array}$ & $\begin{array}{l}4.51 \\
4.34 \\
4.38 \\
3.64 \\
4.57\end{array}$ & $\begin{array}{l}6.07 \\
6.47 \\
6.50 \\
5.09 \\
6.67\end{array}$ \\
\hline & & 1985 & 4.40 & 7.09 \\
\hline \multirow[t]{3}{*}{ SB Raritan River at Three Bridges } & 01397400 & $\begin{array}{l}1975 \\
1976 \\
1977 \\
1978 \\
1979\end{array}$ & $\begin{array}{l}3.64 \\
3.58 \\
3.92 \\
3.19 \\
3.43\end{array}$ & $\begin{array}{l}4.48 \\
4.61 \\
4.48 \\
4.23 \\
4.56\end{array}$ \\
\hline & & $\begin{array}{l}1980 \\
1981 \\
1982 \\
1983 \\
1984\end{array}$ & $\begin{array}{l}3.38 \\
3.25 \\
3.29 \\
2.73 \\
3.43\end{array}$ & $\begin{array}{l}4.55 \\
4.86 \\
4.88 \\
3.82 \\
5.00\end{array}$ \\
\hline & & 1985 & 3.30 & 5.32 \\
\hline
\end{tabular}


Table 16. Estimated application of phosphorus and nitrogen fertilizer in the New Jersey drainage basins studied, 1975-85--Continued

[Based on data supplied by R.B. Alexander, U.S. Geological Survey, written commun., 1989]

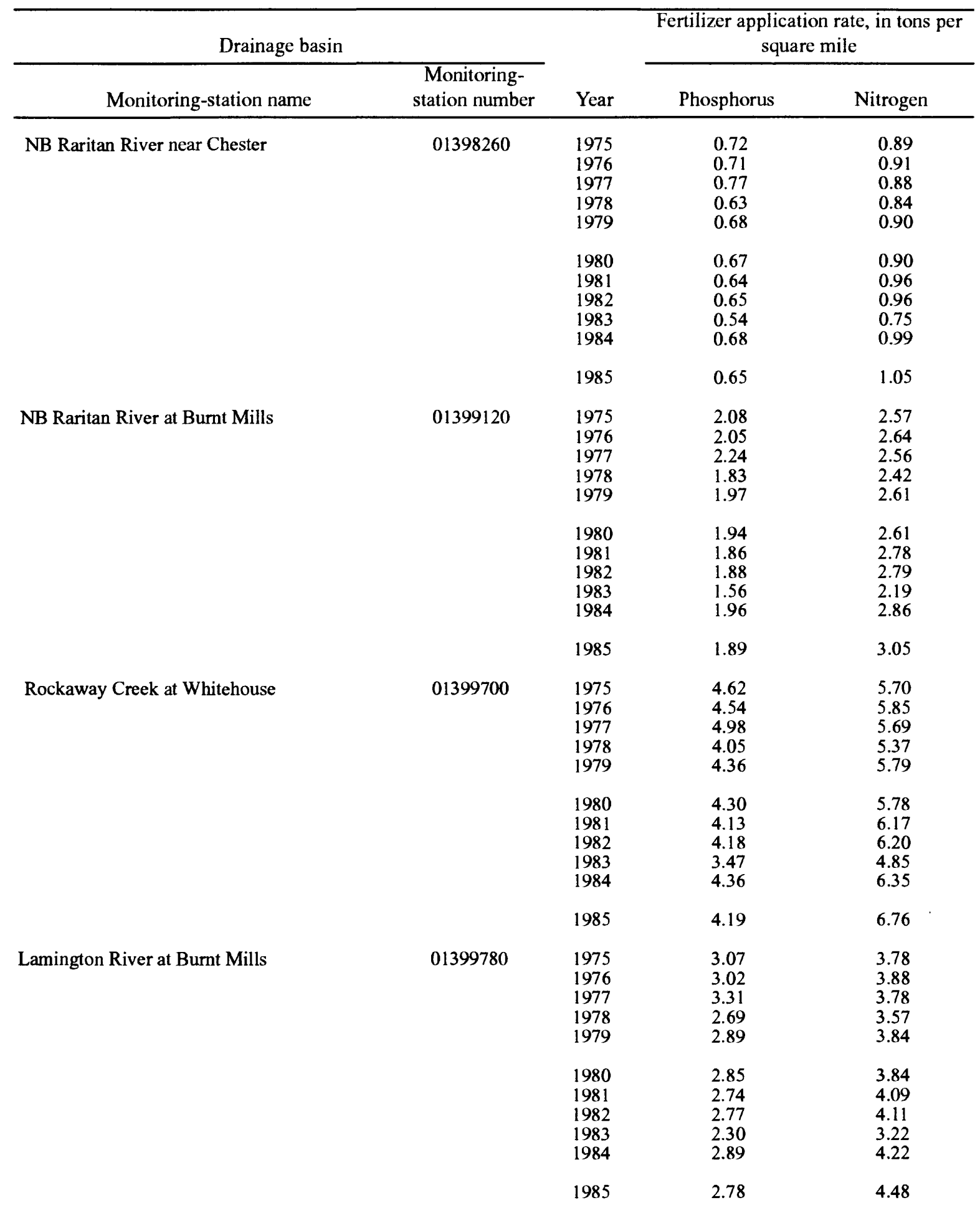


Table 16. Estimated application of phosphorus and nitrogen fertilizer in the New Jersey drainage basins studied, 1975-85--Continued

[Based on data supplied by R.B. Alexander, U.S. Geological Survey, written commun.,1989]

\begin{tabular}{|c|c|c|c|c|}
\hline \multicolumn{2}{|c|}{ Drainage basin } & \multirow[b]{2}{*}{ Year } & \multicolumn{2}{|c|}{$\begin{array}{c}\text { Fertilizer application rate, in tons per } \\
\text { square mile }\end{array}$} \\
\hline Monitoring-station name & $\begin{array}{l}\text { Monitoring- } \\
\text { station number }\end{array}$ & & Phosphorus & Nitrogen \\
\hline \multirow[t]{3}{*}{ Raritan River at Manville } & 01400500 & $\begin{array}{l}1975 \\
1976 \\
1977 \\
1978 \\
1979\end{array}$ & $\begin{array}{l}3.46 \\
3.41 \\
3.73 \\
3.04 \\
3.27\end{array}$ & $\begin{array}{l}4.27 \\
4.39 \\
4.27 \\
4.03 \\
4.34\end{array}$ \\
\hline & & $\begin{array}{l}1980 \\
1981 \\
1982 \\
1983 \\
1984\end{array}$ & $\begin{array}{l}3.22 \\
3.10 \\
3.13 \\
2.60 \\
3.27\end{array}$ & $\begin{array}{l}4.34 \\
4.63 \\
4.65 \\
3.64 \\
4.76\end{array}$ \\
\hline & · & 1985 & 3.14 & 5.07 \\
\hline \multirow[t]{3}{*}{ Millstone River at Grovers Mill } & 01400650 & $\begin{array}{l}1975 \\
1976 \\
1977 \\
1978 \\
1979\end{array}$ & $\begin{array}{l}3.80 \\
3.74 \\
4.10 \\
3.34 \\
3.59\end{array}$ & $\begin{array}{l}4.69 \\
4.82 \\
4.69 \\
4.43 \\
4.77\end{array}$ \\
\hline & & $\begin{array}{l}1980 \\
1981 \\
1982 \\
1983 \\
1984\end{array}$ & $\begin{array}{l}3.54 \\
3.40 \\
3.44 \\
2.86 \\
3.59\end{array}$ & $\begin{array}{l}4.76 \\
5.08 \\
5.10 \\
4.00 \\
5.23\end{array}$ \\
\hline & & 1985 & 3.45 & 5.56 \\
\hline \multirow[t]{3}{*}{ Millstone River at Kingston } & 01401440 & $\begin{array}{l}1975 \\
1976 \\
1977 \\
1978 \\
1979\end{array}$ & $\begin{array}{l}4.15 \\
4.09 \\
4.48 \\
3.65 \\
3.92\end{array}$ & $\begin{array}{l}5.12 \\
5.27 \\
5.12 \\
4.84 \\
5.21\end{array}$ \\
\hline & & $\begin{array}{l}1980 \\
1981 \\
1982 \\
1983 \\
1984\end{array}$ & $\begin{array}{l}3.87 \\
3.72 \\
3.76 \\
3.12 \\
3.92\end{array}$ & $\begin{array}{l}5.20 \\
5.55 \\
5.58 \\
4.37 \\
5.72\end{array}$ \\
\hline & & 1985 & 3.77 & 6.08 \\
\hline \multirow[t]{3}{*}{ Beden Brook near Rocky Hill } & 01401600 & $\begin{array}{l}1975 \\
1976 \\
1977 \\
1978 \\
1979\end{array}$ & $\begin{array}{l}3.97 \\
3.90 \\
4.28 \\
3.48 \\
3.75\end{array}$ & $\begin{array}{l}4.89 \\
5.03 \\
4.89 \\
4.62 \\
4.97\end{array}$ \\
\hline & & $\begin{array}{l}1980 \\
1981 \\
1982 \\
1983 \\
1984\end{array}$ & $\begin{array}{l}3.69 \\
3.55 \\
3.59 \\
2.98 \\
3.74\end{array}$ & $\begin{array}{l}4.97 \\
5.30 \\
5.32 \\
4.17 \\
5.45\end{array}$ \\
\hline & & 1985 & 3.60 & 5.80 \\
\hline
\end{tabular}


Table 16. Estimated application of phosphorus and nitrogen fertilizer in the New Jersey drainage basins studied, 1975-85--Continued

[Based on data supplied by R.B. Alexander, U.S. Geological Survey, written commun., 1989]

\begin{tabular}{|c|c|c|c|c|}
\hline \multicolumn{2}{|l|}{ Drainage basin } & \multirow[b]{2}{*}{ Year } & \multicolumn{2}{|c|}{$\begin{array}{l}\text { Fertilizer application rate, in tons per } \\
\text { square mile }\end{array}$} \\
\hline Monitoring-station name & $\begin{array}{l}\text { Monitoring- } \\
\text { station number }\end{array}$ & & Phosphorus & Nitrogen \\
\hline \multirow[t]{3}{*}{ Millstone River at Weston } & 01402540 & $\begin{array}{l}1975 \\
1976 \\
1977 \\
1978 \\
1979\end{array}$ & $\begin{array}{l}3.88 \\
3.82 \\
4.18 \\
3.40 \\
3.66\end{array}$ & $\begin{array}{l}4.78 \\
4.91 \\
4.78 \\
4.51 \\
4.86\end{array}$ \\
\hline & & $\begin{array}{l}1980 \\
1981 \\
1982 \\
1983 \\
1984\end{array}$ & $\begin{array}{l}3.61 \\
3.47 \\
3.51 \\
2.91 \\
3.66\end{array}$ & $\begin{array}{l}4.85 \\
5.18 \\
5.20 \\
4.07 \\
5.33\end{array}$ \\
\hline & & 1985 & 3.52 & 5.67 \\
\hline \multirow[t]{3}{*}{ Manalapan Brook at Federal Rd near Manalapan } & 01405340 & $\begin{array}{l}1975 \\
1976 \\
1977 \\
1978 \\
1979\end{array}$ & $\begin{array}{l}3.22 \\
3.17 \\
3.47 \\
2.83 \\
3.04\end{array}$ & $\begin{array}{r}3.97 \\
4.08 \\
3.97 \\
3.75 \\
4.04\end{array}$ \\
\hline & & $\begin{array}{l}1980 \\
1981 \\
1982 \\
1983 \\
1984\end{array}$ & $\begin{array}{l}3.00 \\
2.88 \\
2.91 \\
2.42 \\
3.04\end{array}$ & $\begin{array}{l}4.03 \\
4.30 \\
4.32 \\
3.39 \\
4.43\end{array}$ \\
\hline & & 1985 & 2.92 & 4.71 \\
\hline \multirow[t]{3}{*}{ Shark River near Neptune City } & 01407705 & $\begin{array}{l}1975 \\
1976 \\
1977 \\
1978 \\
1979\end{array}$ & $\begin{array}{l}3.62 \\
3.56 \\
3.91 \\
3.18 \\
3.42\end{array}$ & $\begin{array}{l}4.47 \\
4.59 \\
4.46 \\
4.22 \\
4.54\end{array}$ \\
\hline & & $\begin{array}{l}1980 \\
1981 \\
1982 \\
1983 \\
1984\end{array}$ & $\begin{array}{l}3.37 \\
3.24 \\
3.28 \\
2.72 \\
3.42\end{array}$ & $\begin{array}{l}4.54 \\
4.84 \\
4.86 \\
3.81 \\
4.98\end{array}$ \\
\hline & & 1985 & 3.29 & 5.30 \\
\hline \multirow[t]{3}{*}{ Jumping Brook near Neptune City } & 01407760 & $\begin{array}{l}1975 \\
1976 \\
1977 \\
1978 \\
1979\end{array}$ & $\begin{array}{l}3.62 \\
3.56 \\
3.91 \\
3.18 \\
3.42\end{array}$ & $\begin{array}{l}4.47 \\
4.59 \\
4.46 \\
4.22 \\
4.54\end{array}$ \\
\hline & & $\begin{array}{l}1980 \\
1981 \\
1982 \\
1983 \\
1984\end{array}$ & $\begin{array}{l}3.37 \\
3.24 \\
3.28 \\
2.72 \\
3.42\end{array}$ & $\begin{array}{l}4.54 \\
4.84 \\
4.86 \\
3.81 \\
4.98\end{array}$ \\
\hline & & 1985 & 3.29 & 5.30 \\
\hline
\end{tabular}


Table 16. Estimated application of phosphorus and nitrogen fertilizer in the New Jersey drainage basins studied, 1975-85--Continued

[Based on data supplied by R.B. Alexander, U.S. Geological Survey, written commun.,1989]

\begin{tabular}{|c|c|c|c|c|}
\hline \multicolumn{2}{|l|}{ Drainage basin } & \multirow[b]{2}{*}{ Year } & \multicolumn{2}{|c|}{$\begin{array}{l}\text { Fertilizer application rate, in tons per } \\
\text { square mile }\end{array}$} \\
\hline Monitoring-station name & $\begin{array}{l}\text { Monitoring- } \\
\text { station number }\end{array}$ & & Phosphorus & Nitrogen \\
\hline \multirow[t]{3}{*}{ Marsh Bog Brook at Squankum } & 01407997 & $\begin{array}{l}1975 \\
1976 \\
1977 \\
1978 \\
1979\end{array}$ & $\begin{array}{l}3.62 \\
3.56 \\
3.91 \\
3.18 \\
3.42\end{array}$ & $\begin{array}{l}4.47 \\
4.59 \\
4.46 \\
4.22 \\
4.54\end{array}$ \\
\hline & & $\begin{array}{l}1980 \\
1981 \\
1982 \\
1983 \\
1984\end{array}$ & $\begin{array}{l}3.37 \\
3.24 \\
3.28 \\
2.72 \\
3.42\end{array}$ & $\begin{array}{l}4.54 \\
4.84 \\
4.86 \\
3.81 \\
4.98\end{array}$ \\
\hline & & 1985 & 3.29 & 5.30 \\
\hline \multirow[t]{3}{*}{ Toms River near Toms River } & 01408500 & $\begin{array}{l}1975 \\
1976 \\
1977 \\
1978 \\
1979\end{array}$ & $\begin{array}{l}0.28 \\
0.28 \\
0.31 \\
0.25 \\
0.27\end{array}$ & $\begin{array}{l}0.35 \\
0.36 \\
0.35 \\
0.33 \\
0.36\end{array}$ \\
\hline & & $\begin{array}{l}1980 \\
1981 \\
1982 \\
1983 \\
1984\end{array}$ & $\begin{array}{l}0.26 \\
0.26 \\
0.26 \\
0.21 \\
0.27\end{array}$ & $\begin{array}{l}0.36 \\
0.38 \\
0.38 \\
0.30 \\
0.39\end{array}$ \\
\hline & & 1985 & 0.26 & 0.42 \\
\hline \multirow[t]{3}{*}{ Mullica River at outlet of Atsion Lake at Atsion } & 01409387 & $\begin{array}{l}1975 \\
1976 \\
1977 \\
1978 \\
1979\end{array}$ & $\begin{array}{l}2.36 \\
2.33 \\
2.55 \\
2.07 \\
2.23\end{array}$ & $\begin{array}{l}2.92 \\
3.00 \\
2.91 \\
2.75 \\
2.97\end{array}$ \\
\hline & & $\begin{array}{l}1980 \\
1981 \\
1982 \\
1983 \\
1984\end{array}$ & $\begin{array}{l}2.20 \\
2.12 \\
2.14 \\
1.78 \\
2.23\end{array}$ & $\begin{array}{l}2.96 \\
3.16 \\
3.17 \\
2.49 \\
3.25\end{array}$ \\
\hline & & 1985 & 2.15 & 3.46 \\
\hline \multirow[t]{3}{*}{ Hammonton Creek at Westcoatville } & 01409416 & $\begin{array}{l}1975 \\
1976 \\
1977 \\
1978 \\
1979\end{array}$ & $\begin{array}{l}0.89 \\
0.88 \\
0.96 \\
0.78 \\
0.84\end{array}$ & $\begin{array}{l}1.10 \\
1.13 \\
1.10 \\
1.04 \\
1.12\end{array}$ \\
\hline & & $\begin{array}{l}1980 \\
1981 \\
1982 \\
1983 \\
1984\end{array}$ & $\begin{array}{l}0.83 \\
0.80 \\
0.81 \\
0.67 \\
0.84\end{array}$ & $\begin{array}{l}1.12 \\
1.19 \\
1.20 \\
0.94 \\
1.23\end{array}$ \\
\hline & & 1985 & 0.81 & 1.31 \\
\hline
\end{tabular}


Table 16. Estimated application of phosphorus and nitrogen fertilizer in the New Jersey drainage basins studied, 1975-85--Continued

[Based on data supplied by R.B. Alexander, U.S. Geological Survey, written commun., 1989]

\begin{tabular}{|c|c|c|c|c|}
\hline \multicolumn{2}{|l|}{ Drainage basin } & \multirow[b]{2}{*}{ Year } & \multicolumn{2}{|c|}{$\begin{array}{l}\text { Fertilizer application rate, in tons per } \\
\text { square mile }\end{array}$} \\
\hline Monitoring-station name & $\begin{array}{l}\text { Monitoring- } \\
\text { station number }\end{array}$ & & Phosphorus & Nitrogen \\
\hline Batsto River at Batsto & 01409500 & $\begin{array}{l}1975 \\
1976 \\
1977 \\
1978 \\
1979\end{array}$ & $\begin{array}{l}3.09 \\
3.04 \\
3.34 \\
2.71 \\
2.92\end{array}$ & $\begin{array}{l}3.81 \\
3.92 \\
3.81 \\
3.60 \\
3.88\end{array}$ \\
\hline \multirow[t]{3}{*}{ Batsto River at Batsto--Continued } & $\begin{array}{l}01409500- \\
\text { Cont. }\end{array}$ & 1980 & 2.88 & 3.87 \\
\hline & & $\begin{array}{l}1981 \\
1982 \\
1983 \\
1984\end{array}$ & $\begin{array}{l}2.77 \\
2.80 \\
2.32 \\
2.92\end{array}$ & $\begin{array}{l}4.13 \\
4.15 \\
3.25 \\
4.25\end{array}$ \\
\hline & & 1985 & 2.81 & 4.52 \\
\hline \multirow[t]{3}{*}{ West Branch Wading River at Maxwell } & 01409815 & $\begin{array}{l}1975 \\
1976 \\
1977 \\
1978 \\
1979\end{array}$ & $\begin{array}{l}3.08 \\
3.03 \\
3.32 \\
2.70 \\
2.91\end{array}$ & $\begin{array}{l}3.80 \\
3.90 \\
3.80 \\
3.59 \\
3.86\end{array}$ \\
\hline & & $\begin{array}{l}1980 \\
1981 \\
1982 \\
1983 \\
1984\end{array}$ & $\begin{array}{l}2.87 \\
2.76 \\
2.79 \\
2.32 \\
2.90\end{array}$ & $\begin{array}{l}3.86 \\
4.11 \\
4.13 \\
3.24 \\
4.24\end{array}$ \\
\hline & & 1985 & 2.80 & 4.51 \\
\hline \multirow[t]{3}{*}{ Oswego River at Harrisville } & 01410000 & $\begin{array}{l}1975 \\
1976 \\
1977 \\
1978 \\
1979\end{array}$ & $\begin{array}{l}1.96 \\
1.93 \\
2.12 \\
1.72 \\
1.85\end{array}$ & $\begin{array}{l}2.42 \\
2.49 \\
2.42 \\
2.28 \\
2.46\end{array}$ \\
\hline & & $\begin{array}{l}1980 \\
1981 \\
1982 \\
1983 \\
1984\end{array}$ & $\begin{array}{l}1.83 \\
1.76 \\
1.77 \\
1.48 \\
1.85\end{array}$ & $\begin{array}{l}2.46 \\
2.62 \\
2.63 \\
2.06 \\
2.70\end{array}$ \\
\hline & & 1985 & 1.78 & 2.87 \\
\hline \multirow[t]{3}{*}{ East Branch Bass River near New Gretna } & 01410150 & $\begin{array}{l}1975 \\
1976 \\
1977 \\
1978 \\
1979\end{array}$ & $\begin{array}{l}2.97 \\
2.92 \\
3.20 \\
2.60 \\
2.80\end{array}$ & $\begin{array}{l}3.66 \\
3.76 \\
3.66 \\
3.46 \\
3.72\end{array}$ \\
\hline & & $\begin{array}{l}1980 \\
1981 \\
1982 \\
1983 \\
1984\end{array}$ & $\begin{array}{l}2.76 \\
2.66 \\
2.69 \\
2.23 \\
2.80\end{array}$ & $\begin{array}{l}3.72 \\
3.96 \\
3.98 \\
3.12 \\
4.08\end{array}$ \\
\hline & & 1985 & 2.69 & 4.34 \\
\hline
\end{tabular}


Table 16. Estimated application of phosphorus and nitrogen fertilizer in the New Jersey drainage basins studied, 1975-85--Continued

[Based on data supplied by R.B. Alexander, U.S. Geological Survey, written commun.,1989]

\begin{tabular}{|c|c|c|c|c|}
\hline \multicolumn{2}{|c|}{ Drainage basin } & \multirow[b]{2}{*}{ Year } & \multicolumn{2}{|c|}{$\begin{array}{l}\text { Fertilizer application rate, in tons per } \\
\text { square mile }\end{array}$} \\
\hline Monitoring-station name & $\begin{array}{l}\text { Monitoring- } \\
\text { station number }\end{array}$ & & Phosphorus & Nitrogen \\
\hline \multirow[t]{3}{*}{ Great Egg Harbor near Sicklerville } & 01410784 & $\begin{array}{l}1975 \\
1976 \\
1977 \\
1978 \\
1979\end{array}$ & $\begin{array}{l}1.51 \\
1.49 \\
1.63 \\
1.33 \\
1.43\end{array}$ & $\begin{array}{l}1.87 \\
1.92 \\
1.87 \\
1.76 \\
1.90\end{array}$ \\
\hline & & $\begin{array}{l}1980 \\
1981 \\
1982 \\
1983 \\
1984\end{array}$ & $\begin{array}{l}1.41 \\
1.35 \\
1.37 \\
1.14 \\
1.43\end{array}$ & $\begin{array}{l}1.90 \\
2.02 \\
2.03 \\
1.59 \\
2.08\end{array}$ \\
\hline & & 1985 & 1.38 & 2.22 \\
\hline \multirow[t]{3}{*}{ Great Harbor near Blue Anchor } & 01410820 & $\begin{array}{l}1975 \\
1976 \\
1977 \\
1978 \\
1979\end{array}$ & $\begin{array}{l}2.16 \\
2.13 \\
2.33 \\
1.90 \\
2.04\end{array}$ & $\begin{array}{l}2.67 \\
2.74 \\
2.66 \\
2.52 \\
2.71\end{array}$ \\
\hline & & $\begin{array}{l}1980 \\
1981 \\
1982 \\
1983 \\
1984\end{array}$ & $\begin{array}{l}2.01 \\
1.93 \\
1.95 \\
1.62 \\
2.04\end{array}$ & $\begin{array}{l}2.70 \\
2.89 \\
2.90 \\
2.27 \\
2.97\end{array}$ \\
\hline & & 1985 & 1.96 & 3.16 \\
\hline \multirow[t]{3}{*}{ Great Egg Harbor at Weymouth } & 01411110 & $\begin{array}{l}1975 \\
1976 \\
1977 \\
1978 \\
1979\end{array}$ & $\begin{array}{l}2.55 \\
2.51 \\
2.75 \\
2.24 \\
2.41\end{array}$ & $\begin{array}{l}3.15 \\
3.24 \\
3.15 \\
2.97 \\
3.20\end{array}$ \\
\hline & & $\begin{array}{l}1980 \\
1981 \\
1982 \\
1983 \\
1984\end{array}$ & $\begin{array}{l}2.37 \\
2.29 \\
2.31 \\
1.92 \\
2.41\end{array}$ & $\begin{array}{l}3.20 \\
3.41 \\
3.43 \\
2.68 \\
3.51\end{array}$ \\
\hline & & 1985 & 2.32 & 3.74 \\
\hline \multirow[t]{3}{*}{ Maurice River at Norma } & 01411500 & $\begin{array}{l}1975 \\
1976 \\
1977 \\
1978 \\
1979\end{array}$ & $\begin{array}{l}5.08 \\
4.99 \\
5.48 \\
4.45 \\
4.80\end{array}$ & $\begin{array}{l}6.26 \\
6.43 \\
6.26 \\
5.91 \\
6.37\end{array}$ \\
\hline & & $\begin{array}{l}1980 \\
1981 \\
1982 \\
1983 \\
1984\end{array}$ & $\begin{array}{l}4.72 \\
4.54 \\
4.59 \\
3.82 \\
4.79\end{array}$ & $\begin{array}{l}6.36 \\
6.78 \\
6.81 \\
5.34 \\
6.98\end{array}$ \\
\hline & & 1985 & 4.61 & 7.43 \\
\hline
\end{tabular}


Table 16. Estimated application of phosphorus and nitrogen fertilizer in the New Jersey drainage basins studied, 1975-85--Continued

[Based on data supplied by R.B. Alexander, U.S. Geological Survey, written commun., 1989]

\begin{tabular}{|c|c|c|c|c|}
\hline \multicolumn{2}{|l|}{ Drainage basin } & \multirow[b]{2}{*}{ Year } & \multicolumn{2}{|c|}{$\begin{array}{c}\text { Fertilizer application rate, in tons per } \\
\text { square mile }\end{array}$} \\
\hline Monitoring-station name & $\begin{array}{l}\text { Monitoring- } \\
\text { station number }\end{array}$ & & Phosphorus & Nitrogen \\
\hline \multirow[t]{3}{*}{ Cohansey River at Seeley } & 01412800 & $\begin{array}{l}1975 \\
1976 \\
1977 \\
1978 \\
1979\end{array}$ & $\begin{array}{l}6.34 \\
6.23 \\
6.83 \\
5.56 \\
5.99\end{array}$ & $\begin{array}{l}7.81 \\
8.03 \\
7.81 \\
7.37 \\
7.95\end{array}$ \\
\hline & & $\begin{array}{l}1980 \\
1981 \\
1982 \\
1983 \\
1984\end{array}$ & $\begin{array}{l}5.89 \\
5.67 \\
5.73 \\
4.76 \\
5.97\end{array}$ & $\begin{array}{l}7.93 \\
8.46 \\
8.50 \\
6.66 \\
8.71\end{array}$ \\
\hline & & 1985 & 5.75 & 9.27 \\
\hline \multirow[t]{3}{*}{ Paulins Kill at Blairstown } & 01443500 & $\begin{array}{l}1975 \\
1976 \\
1977 \\
1978 \\
1979\end{array}$ & $\begin{array}{l}1.75 \\
1.72 \\
1.89 \\
1.54 \\
1.65\end{array}$ & $\begin{array}{l}2.16 \\
2.22 \\
2.16 \\
2.04 \\
2.20\end{array}$ \\
\hline & & $\begin{array}{l}1980 \\
1981 \\
1982 \\
1983 \\
1984\end{array}$ & $\begin{array}{l}1.63 \\
1.57 \\
1.58 \\
1.32 \\
1.65\end{array}$ & $\begin{array}{l}2.19 \\
2.34 \\
2.35 \\
1.84 \\
2.41\end{array}$ \\
\hline & & 1985 & 1.59 & 2.56 \\
\hline \multirow[t]{3}{*}{ Musconetcong River Outlet of Lake Hopatcong } & 01455500 & $\begin{array}{l}1975 \\
1976 \\
1977 \\
1978 \\
1979\end{array}$ & $\begin{array}{l}0.94 \\
0.92 \\
1.01 \\
0.82 \\
0.88\end{array}$ & $\begin{array}{l}1.15 \\
1.19 \\
1.15 \\
1.09 \\
1.17\end{array}$ \\
\hline & & $\begin{array}{l}1980 \\
1981 \\
1982 \\
1983 \\
1984\end{array}$ & $\begin{array}{l}0.87 \\
0.84 \\
0.85 \\
0.70 \\
0.88\end{array}$ & $\begin{array}{l}1.17 \\
1.25 \\
1.26 \\
0.98 \\
1.29\end{array}$ \\
\hline & & 1985 & 0.85 & 1.37 \\
\hline \multirow[t]{3}{*}{ Musconetcong River at Beattystown } & 01456200 & $\begin{array}{l}1975 \\
1976 \\
1977 \\
1978 \\
1979\end{array}$ & $\begin{array}{l}1.40 \\
1.38 \\
1.51 \\
1.23 \\
1.33\end{array}$ & $\begin{array}{l}1.73 \\
1.78 \\
1.73 \\
1.64 \\
1.76\end{array}$ \\
\hline & & $\begin{array}{l}1980 \\
1981 \\
1982 \\
1983 \\
1984\end{array}$ & $\begin{array}{l}1.31 \\
1.26 \\
1.27 \\
1.06 \\
1.32\end{array}$ & $\begin{array}{l}1.76 \\
1.88 \\
1.89 \\
1.48 \\
1.93\end{array}$ \\
\hline & & 1985 & 1.28 & 2.06 \\
\hline
\end{tabular}


Table 16. Estimated application of phosphorus and nitrogen fertilizer in the New Jersey drainage basins studied, 1975-85--Continued

[Based on data supplied by R.B. Alexander, U.S. Geological Survey, written commun.,1989]

\begin{tabular}{|c|c|c|c|c|}
\hline \multicolumn{2}{|c|}{ Drainage basin } & \multirow[b]{2}{*}{ Year } & \multicolumn{2}{|c|}{$\begin{array}{c}\text { Fertilizer application rate, in tons per } \\
\text { square mile }\end{array}$} \\
\hline Monitoring-station name & $\begin{array}{l}\text { Monitoring- } \\
\text { station number }\end{array}$ & & Phosphorus & Nitrogen \\
\hline \multirow[t]{3}{*}{ Musconetcong River at Riegelsville } & 01457400 & $\begin{array}{l}1975 \\
1976 \\
1977 \\
1978 \\
1979\end{array}$ & $\begin{array}{l}2.93 \\
2.89 \\
3.16 \\
2.57 \\
2.77\end{array}$ & $\begin{array}{l}3.62 \\
3.72 \\
3.62 \\
3.41 \\
3.68\end{array}$ \\
\hline & & $\begin{array}{l}1980 \\
1981 \\
1982 \\
1983 \\
1984\end{array}$ & $\begin{array}{l}2.73 \\
2.63 \\
2.65 \\
2.21 \\
2.77\end{array}$ & $\begin{array}{l}3.67 \\
3.92 \\
3.94 \\
3.08 \\
4.04\end{array}$ \\
\hline & & 1985 & 2.66 & 4.29 \\
\hline \multirow[t]{3}{*}{ Wickechcoke Creek at Stockton } & 01461300 & $\begin{array}{l}1975 \\
1976 \\
1977 \\
1978 \\
1979\end{array}$ & $\begin{array}{l}4.85 \\
4.77 \\
5.23 \\
4.25 \\
4.58\end{array}$ & $\begin{array}{l}5.98 \\
6.14 \\
5.97 \\
5.64 \\
6.08\end{array}$ \\
\hline & & $\begin{array}{l}1980 \\
1981 \\
1982 \\
1983 \\
1984\end{array}$ & $\begin{array}{l}4.51 \\
4.34 \\
4.38 \\
3.64 \\
4.57\end{array}$ & $\begin{array}{l}6.07 \\
6.47 \\
6.50 \\
5.09 \\
6.67\end{array}$ \\
\hline & & 1985 & 4.40 & 7.09 \\
\hline \multirow[t]{3}{*}{ Crosswicks Creek at Extonville } & 01464500 & $\begin{array}{l}1975 \\
1976 \\
1977 \\
1978 \\
1979\end{array}$ & $\begin{array}{l}2.05 \\
2.01 \\
2.21 \\
1.79 \\
1.93\end{array}$ & $\begin{array}{l}2.52 \\
2.59 \\
2.52 \\
2.38 \\
2.57\end{array}$ \\
\hline & & $\begin{array}{l}1980 \\
1981 \\
1982 \\
1983 \\
1984\end{array}$ & $\begin{array}{l}1.90 \\
1.83 \\
1.85 \\
1.54 \\
1.93\end{array}$ & $\begin{array}{l}2.56 \\
2.73 \\
2.75 \\
2.15 \\
2.81\end{array}$ \\
\hline & & 1985 & 1.86 & 2.99 \\
\hline \multirow[t]{3}{*}{ Doctors Creek at Allentown } & 01464515 & $\begin{array}{l}1975 \\
1976 \\
1977 \\
1978 \\
1979\end{array}$ & $\begin{array}{l}3.63 \\
3.58 \\
3.92 \\
3.19 \\
3.43\end{array}$ & $\begin{array}{l}4.48 \\
4.61 \\
4.48 \\
4.23 \\
4.56\end{array}$ \\
\hline & & $\begin{array}{l}1980 \\
1981 \\
1982 \\
1983 \\
1984\end{array}$ & $\begin{array}{l}3.38 \\
3.25 \\
3.29 \\
2.73 \\
3.43\end{array}$ & $\begin{array}{l}4.55 \\
4.85 \\
4.88 \\
3.82 \\
5.00\end{array}$ \\
\hline & & 1985 & 3.30 & 5.32 \\
\hline
\end{tabular}


Table 16. Estimated application of phosphorus and nitrogen fertilizer in the New Jersey drainage basins studied, 1975-85--Continued

[Based on data supplied by R.B. Alexander, U.S. Geological Survey, written commun.,1989]

\begin{tabular}{|c|c|c|c|c|}
\hline \multicolumn{2}{|l|}{ Drainage basin } & \multirow[b]{2}{*}{ Year } & \multicolumn{2}{|c|}{$\begin{array}{c}\text { Fertilizer application rate, in tons per } \\
\text { square mile }\end{array}$} \\
\hline Monitoring-station name & $\begin{array}{l}\text { Monitoring- } \\
\text { station number }\end{array}$ & & Phosphorus & Nitrogen \\
\hline \multirow[t]{3}{*}{ SB Rancocas Creek at Vincentown } & 01465850 & $\begin{array}{l}1975 \\
1976 \\
1977 \\
1978 \\
1979\end{array}$ & $\begin{array}{l}3.09 \\
3.04 \\
3.34 \\
2.71 \\
2.92\end{array}$ & $\begin{array}{l}3.81 \\
3.92 \\
3.81 \\
3.60 \\
3.88\end{array}$ \\
\hline & & $\begin{array}{l}1980 \\
1981 \\
1982 \\
1983 \\
1984\end{array}$ & $\begin{array}{l}2.88 \\
2.77 \\
2.80 \\
2.32 \\
2.92\end{array}$ & $\begin{array}{l}3.87 \\
4.13 \\
4.15 \\
3.25 \\
4.25\end{array}$ \\
\hline & & 1985 & 2.81 & 4.52 \\
\hline \multirow[t]{3}{*}{ NB Rancocas Creek at Brown Mills } & 01465970 & $\begin{array}{l}1975 \\
1976 \\
1977 \\
1978 \\
1979\end{array}$ & $\begin{array}{l}1.93 \\
1.90 \\
2.08 \\
1.69 \\
1.82\end{array}$ & $\begin{array}{l}2.38 \\
2.44 \\
2.38 \\
2.24 \\
2.42\end{array}$ \\
\hline & & $\begin{array}{l}1980 \\
1981 \\
1982 \\
1983 \\
1984\end{array}$ & $\begin{array}{l}1.79 \\
1.73 \\
1.74 \\
1.45 \\
1.82\end{array}$ & $\begin{array}{l}2.41 \\
2.58 \\
2.59 \\
2.03 \\
2.65\end{array}$ \\
\hline & & 1985 & 1.75 & 2.82 \\
\hline \multirow[t]{3}{*}{ McDonalds Branch in Lebanon State Forest } & 01466500 & $\begin{array}{l}1975 \\
1976 \\
1977 \\
1978 \\
1979\end{array}$ & $\begin{array}{l}3.09 \\
3.04 \\
3.34 \\
2.71 \\
2.92\end{array}$ & $\begin{array}{l}3.81 \\
3.92 \\
3.81 \\
3.60 \\
3.88\end{array}$ \\
\hline & & $\begin{array}{l}1980 \\
1981 \\
1982 \\
1983 \\
1984\end{array}$ & $\begin{array}{l}2.88 \\
2.77 \\
2.80 \\
2.32 \\
2.92\end{array}$ & $\begin{array}{l}3.87 \\
4.13 \\
4.15 \\
3.25 \\
4.25\end{array}$ \\
\hline & & 1985 & 2.81 & 4.52 \\
\hline \multirow[t]{3}{*}{ NB Rancocas Creek at Browns Mills } & 01467000 & $\begin{array}{l}1975 \\
1976 \\
1977 \\
1978 \\
1979\end{array}$ & $\begin{array}{l}2.02 \\
1.99 \\
2.18 \\
1.77 \\
1.91\end{array}$ & $\begin{array}{l}2.49 \\
2.56 \\
2.49 \\
2.35 \\
2.53\end{array}$ \\
\hline & & $\begin{array}{l}1980 \\
1981 \\
1982 \\
1983 \\
1984\end{array}$ & $\begin{array}{l}1.88 \\
1.81 \\
1.83 \\
1.52 \\
1.91\end{array}$ & $\begin{array}{l}2.53 \\
2.70 \\
2.71 \\
2.12 \\
2.78\end{array}$ \\
\hline & & 1985 & 1.83 & 2.96 \\
\hline
\end{tabular}


Table 16. Estimated application of phosphorus and nitrogen fertilizer in the New Jersey drainage basins studied, 1975-85--Continued

[Based on data supplied by R.B. Alexander, U.S. Geological Survey, written commun., 1989]

\begin{tabular}{|c|c|c|c|c|}
\hline \multicolumn{2}{|l|}{ Drainage basin } & \multirow[b]{2}{*}{ Year } & \multicolumn{2}{|c|}{$\begin{array}{l}\text { Fertilizer application rate, in tons per } \\
\text { square mile }\end{array}$} \\
\hline Monitoring-station name & $\begin{array}{l}\text { Monitoring- } \\
\text { station number }\end{array}$ & & Phosphorus & Nitrogen \\
\hline \multirow[t]{3}{*}{ NB Pennsauken Creek near Moorestown } & 01467069 & $\begin{array}{l}1975 \\
1976 \\
1977 \\
1978 \\
1979\end{array}$ & $\begin{array}{l}3.09 \\
3.04 \\
3.34 \\
2.71 \\
2.92\end{array}$ & $\begin{array}{l}3.81 \\
3.92 \\
3.81 \\
3.60 \\
3.88\end{array}$ \\
\hline & & $\begin{array}{l}1980 \\
1981 \\
1982 \\
1983 \\
1984\end{array}$ & $\begin{array}{l}2.88 \\
2.77 \\
2.80 \\
2.32 \\
2.92\end{array}$ & $\begin{array}{l}3.87 \\
4.13 \\
4.15 \\
3.25 \\
4.25\end{array}$ \\
\hline & & 1985 & 2.81 & 4.52 \\
\hline \multirow[t]{3}{*}{ SB Pennsauken Creek at Cherry Hill } & 01467081 & $\begin{array}{l}1975 \\
1976 \\
1977 \\
1978 \\
1979\end{array}$ & $\begin{array}{l}2.01 \\
1.97 \\
2.16 \\
1.76 \\
1.90\end{array}$ & $\begin{array}{l}2.48 \\
2.54 \\
2.47 \\
2.34 \\
2.52\end{array}$ \\
\hline & & $\begin{array}{l}1980 \\
1981 \\
1982 \\
1983 \\
1984\end{array}$ & $\begin{array}{l}1.87 \\
1.80 \\
1.81 \\
1.51 \\
1.89\end{array}$ & $\begin{array}{l}2.51 \\
2.68 \\
2.69 \\
2.11 \\
2.76\end{array}$ \\
\hline & & 1985 & 1.82 & 2.94 \\
\hline \multirow[t]{3}{*}{ Cooper River at Norcross Rd at Lindenwold } & 01467120 & $\begin{array}{l}1975 \\
1976 \\
1977 \\
1978 \\
1979\end{array}$ & $\begin{array}{l}1.51 \\
1.49 \\
1.63 \\
1.33 \\
1.43\end{array}$ & $\begin{array}{l}1.87 \\
1.92 \\
1.87 \\
1.76 \\
1.90\end{array}$ \\
\hline & & $\begin{array}{l}1980 \\
1981 \\
1982 \\
1983 \\
1984\end{array}$ & $\begin{array}{l}1.41 \\
1.35 \\
1.37 \\
1.14 \\
1.43\end{array}$ & $\begin{array}{l}1.90 \\
2.02 \\
2.03 \\
1.59 \\
2.08\end{array}$ \\
\hline & & 1985 & 1.38 & 2.22 \\
\hline \multirow[t]{3}{*}{ Cooper River at Lawnside } & 01467140 & $\begin{array}{l}1975 \\
1976 \\
1977 \\
1978 \\
1979\end{array}$ & $\begin{array}{l}1.51 \\
1.49 \\
1.63 \\
1.33 \\
1.43\end{array}$ & $\begin{array}{l}1.87 \\
1.92 \\
1.87 \\
1.76 \\
1.90\end{array}$ \\
\hline & & $\begin{array}{l}1980 \\
1981 \\
1982 \\
1983 \\
1984\end{array}$ & $\begin{array}{l}1.41 \\
1.35 \\
1.37 \\
1.14 \\
1.43\end{array}$ & $\begin{array}{l}1.90 \\
2.02 \\
2.03 \\
1.59 \\
2.08\end{array}$ \\
\hline & & 1985 & 1.38 & 2.22 \\
\hline
\end{tabular}


Table 16. Estimated application of phosphorus and nitrogen fertilizer in the New Jersey drainage basins studied, 1975-85--Continued

[Based on data supplied by R.B. Alexander, U.S. Geological Survey, written commun.,1989]

\begin{tabular}{|c|c|c|c|c|}
\hline \multicolumn{2}{|l|}{ Drainage basin } & \multirow[b]{2}{*}{ Year } & \multicolumn{2}{|c|}{$\begin{array}{l}\text { Fertilizer application rate, in tons per } \\
\text { square mile }\end{array}$} \\
\hline Monitoring-station name & $\begin{array}{l}\text { Monitoring- } \\
\text { station number }\end{array}$ & & Phosphorus & Nitrogen \\
\hline \multirow[t]{3}{*}{ SB Big Timber Creek at Blackwood Terrace } & 01467329 & $\begin{array}{l}1975 \\
1976 \\
1977 \\
1978 \\
1979\end{array}$ & $\begin{array}{l}2.88 \\
2.84 \\
3.11 \\
2.53 \\
2.72\end{array}$ & $\begin{array}{l}3.56 \\
3.65 \\
3.55 \\
3.36 \\
3.62\end{array}$ \\
\hline & & $\begin{array}{l}1980 \\
1981 \\
1982 \\
1983 \\
1984\end{array}$ & $\begin{array}{l}2.68 \\
2.58 \\
2.61 \\
2.17 \\
2.72\end{array}$ & $\begin{array}{l}3.61 \\
3.85 \\
3.87 \\
3.03 \\
3.97\end{array}$ \\
\hline & & 1985 & 2.62 & 4.22 \\
\hline \multirow[t]{3}{*}{ Raccoon Creek near Swedesboro } & 01477120 & $\begin{array}{l}1975 \\
1976 \\
1977 \\
1978 \\
1979\end{array}$ & $\begin{array}{l}4.90 \\
4.82 \\
5.28 \\
4.30 \\
4.63\end{array}$ & $\begin{array}{l}6.04 \\
6.21 \\
6.04 \\
5.70 \\
6.14\end{array}$ \\
\hline & & $\begin{array}{l}1980 \\
1981 \\
1982 \\
1983 \\
1984\end{array}$ & $\begin{array}{l}4.56 \\
4.38 \\
4.43 \\
3.68 \\
4.62\end{array}$ & $\begin{array}{l}6.13 \\
6.54 \\
6.57 \\
5.15 \\
6.74\end{array}$ \\
\hline & & 1985 & 4.45 & 7.17 \\
\hline \multirow[t]{3}{*}{ Oldmans Creek at Porches Mill } & 01477510 & $\begin{array}{l}1975 \\
1976 \\
1977 \\
1978 \\
1979\end{array}$ & $\begin{array}{l}6.13 \\
6.03 \\
6.61 \\
5.38 \\
5.79\end{array}$ & $\begin{array}{l}7.56 \\
7.77 \\
7.55 \\
7.13 \\
7.68\end{array}$ \\
\hline & & $\begin{array}{l}1980 \\
1981 \\
1982 \\
1983 \\
1984\end{array}$ & $\begin{array}{l}5.70 \\
5.48 \\
5.54 \\
4.61 \\
5.78\end{array}$ & $\begin{array}{l}7.67 \\
8.18 \\
8.22 \\
6.44 \\
8.43\end{array}$ \\
\hline & & 1985 & 5.56 & 8.97 \\
\hline \multirow[t]{3}{*}{ Salem River at Woodstown } & 01482500 & $\begin{array}{l}1975 \\
1976 \\
1977 \\
1978 \\
1979\end{array}$ & $\begin{array}{l}7.56 \\
7.44 \\
8.16 \\
6.64 \\
7.15\end{array}$ & $\begin{array}{l}9.33 \\
9.59 \\
9.32 \\
8.80 \\
9.49\end{array}$ \\
\hline & & $\begin{array}{l}1980 \\
1981 \\
1982 \\
1983 \\
1984\end{array}$ & $\begin{array}{l}7.04 \\
6.77 \\
6.84 \\
5.69 \\
7.13\end{array}$ & $\begin{array}{r}9.47 \\
10.10 \\
10.15 \\
7.95 \\
10.40\end{array}$ \\
\hline & & 1985 & 6.87 & 11.07 \\
\hline
\end{tabular}




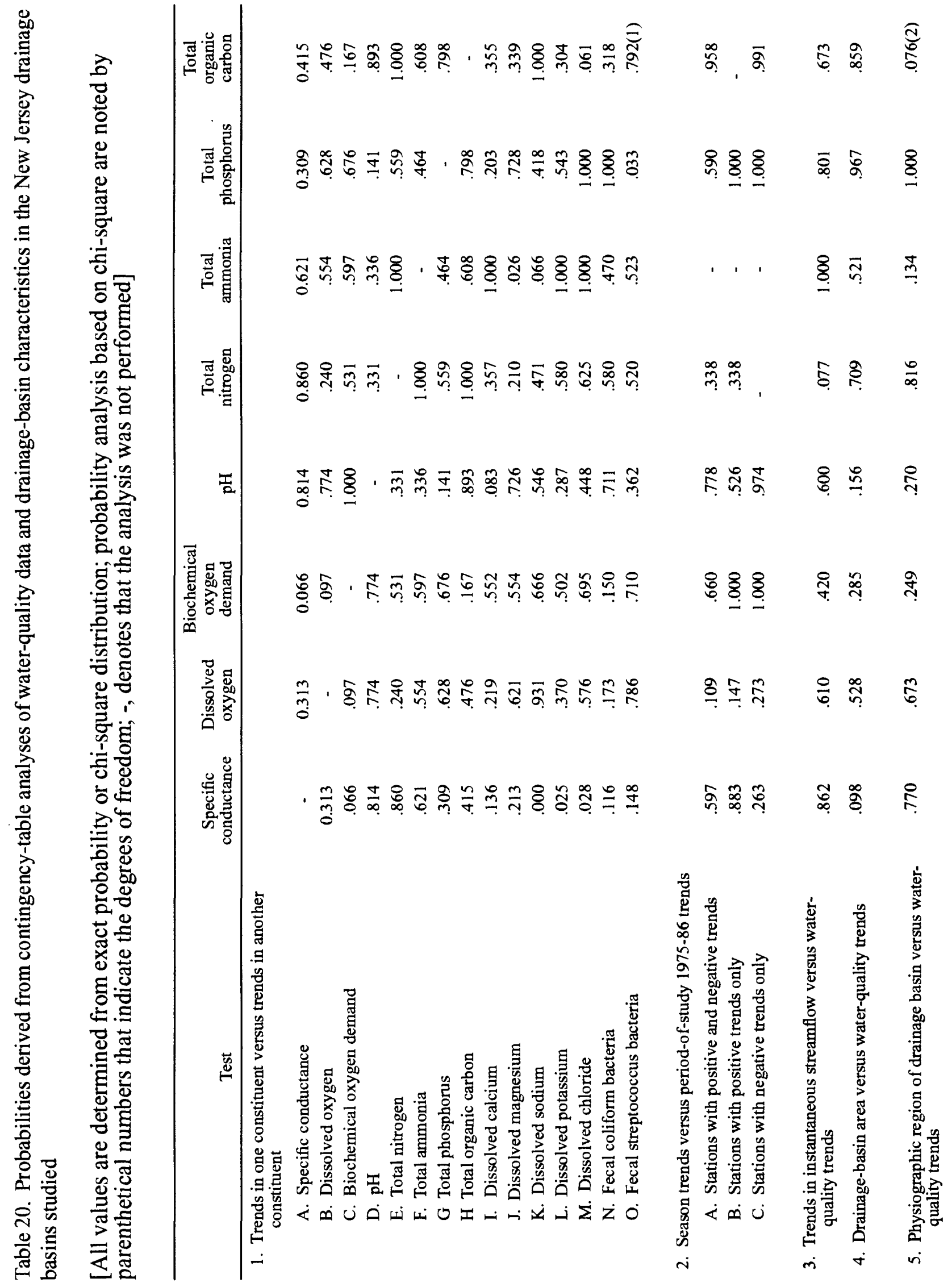




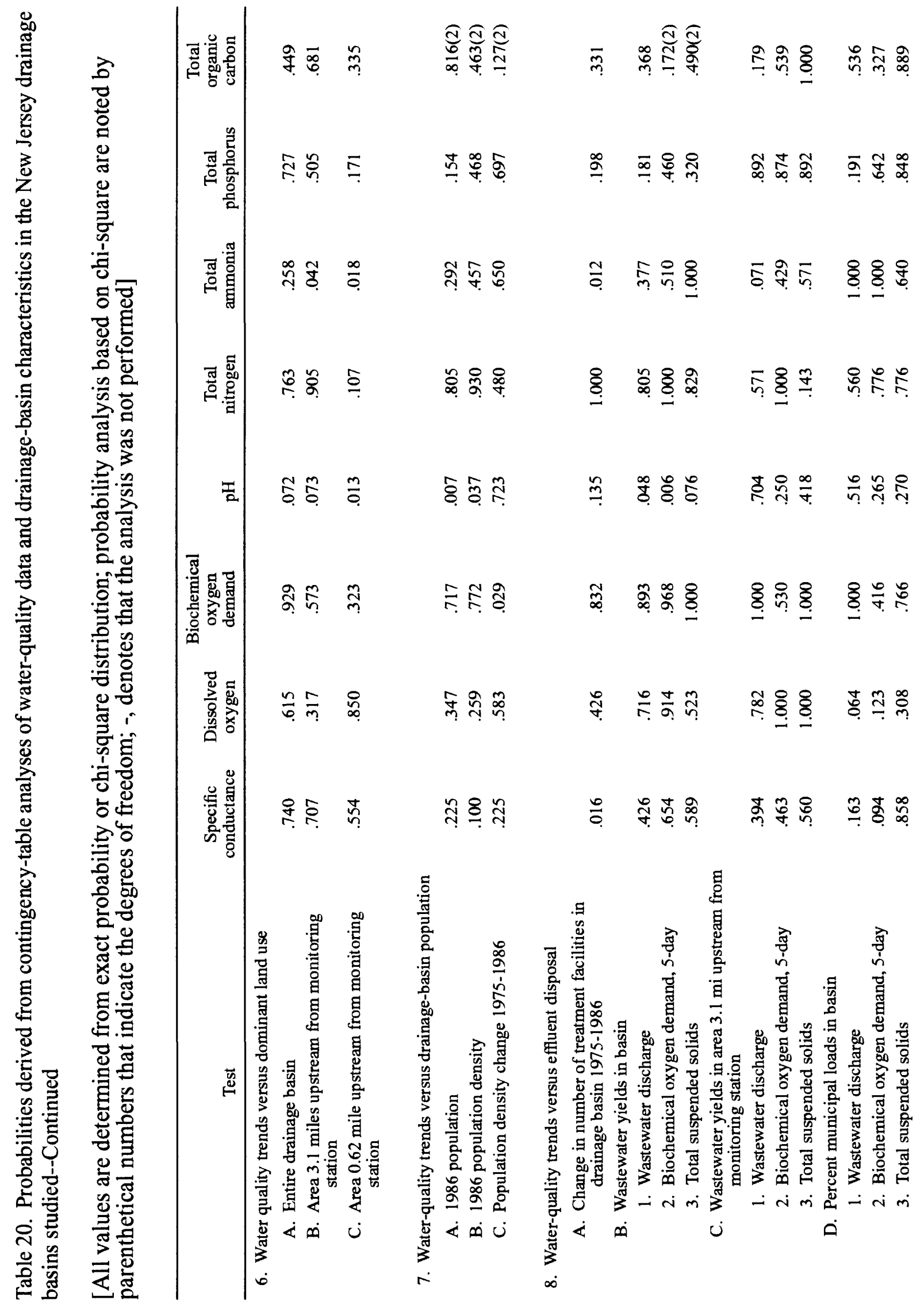




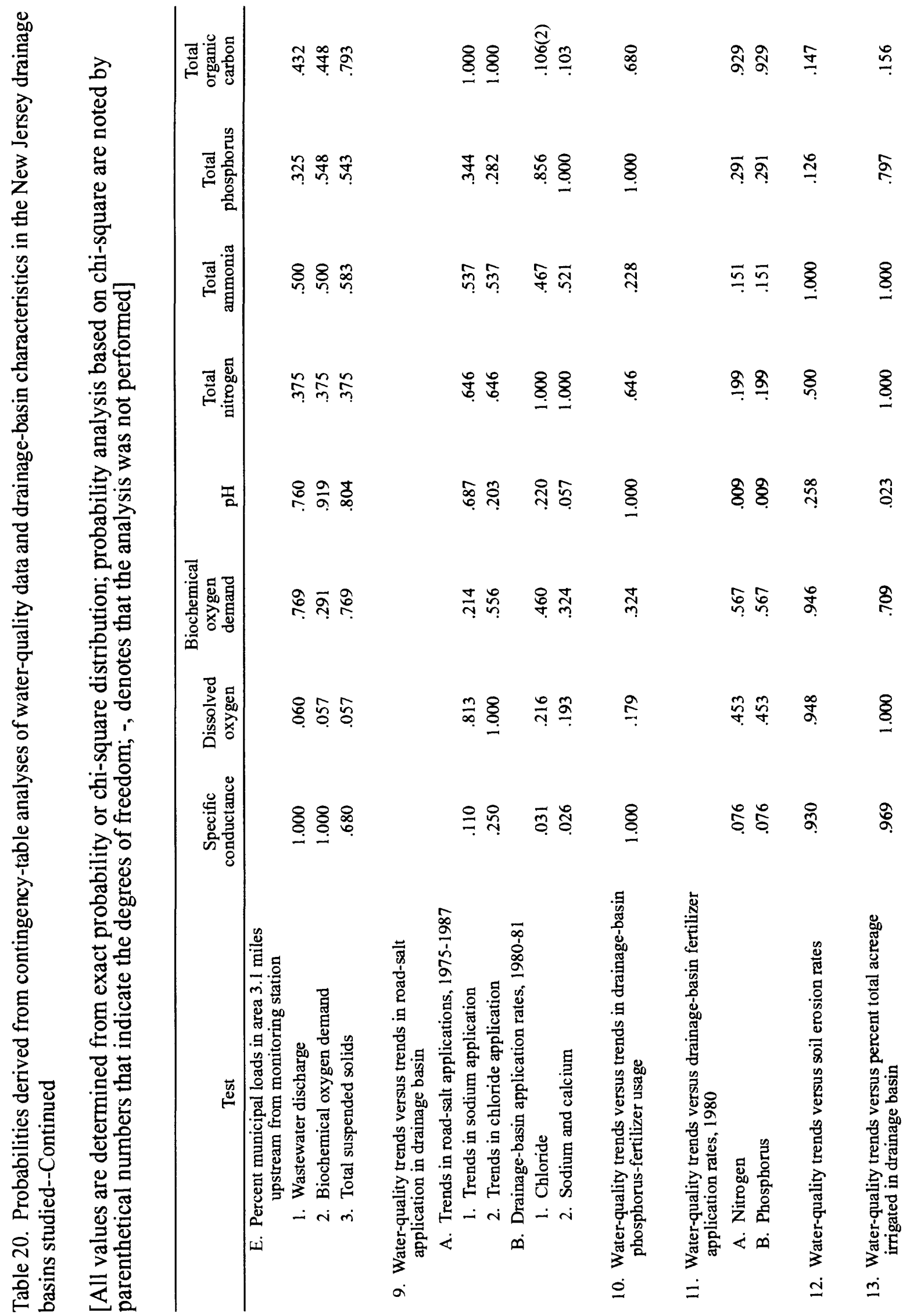




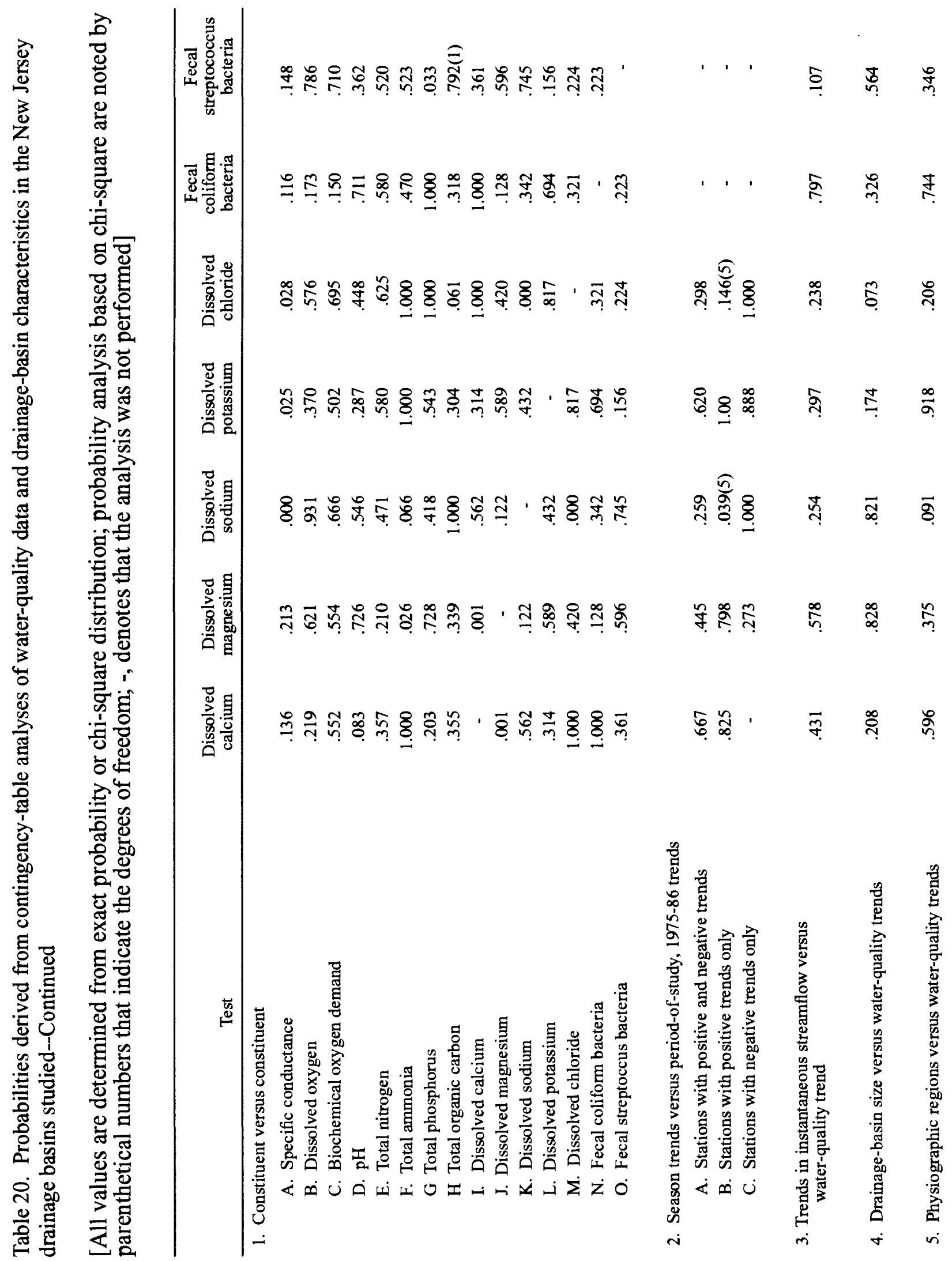




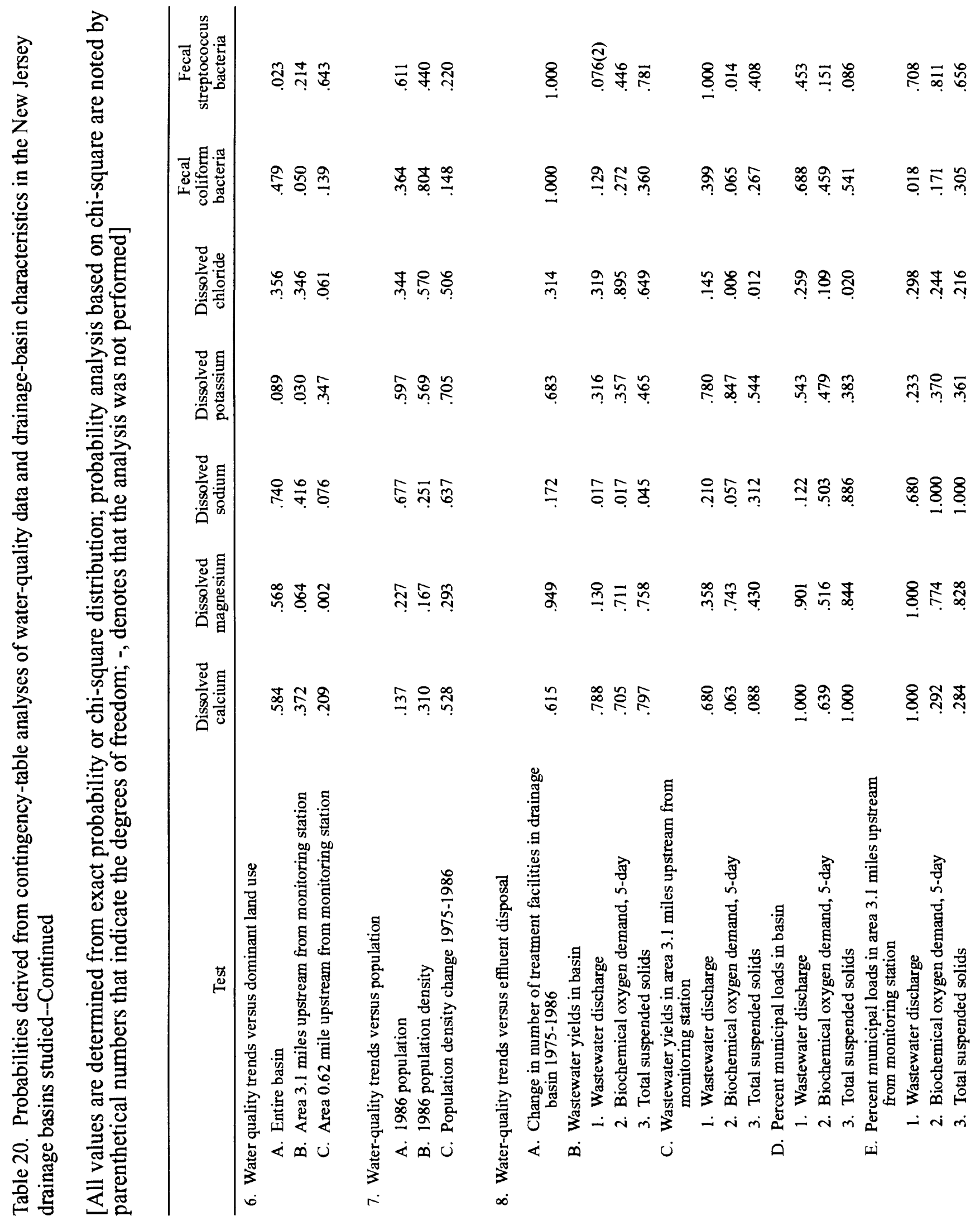




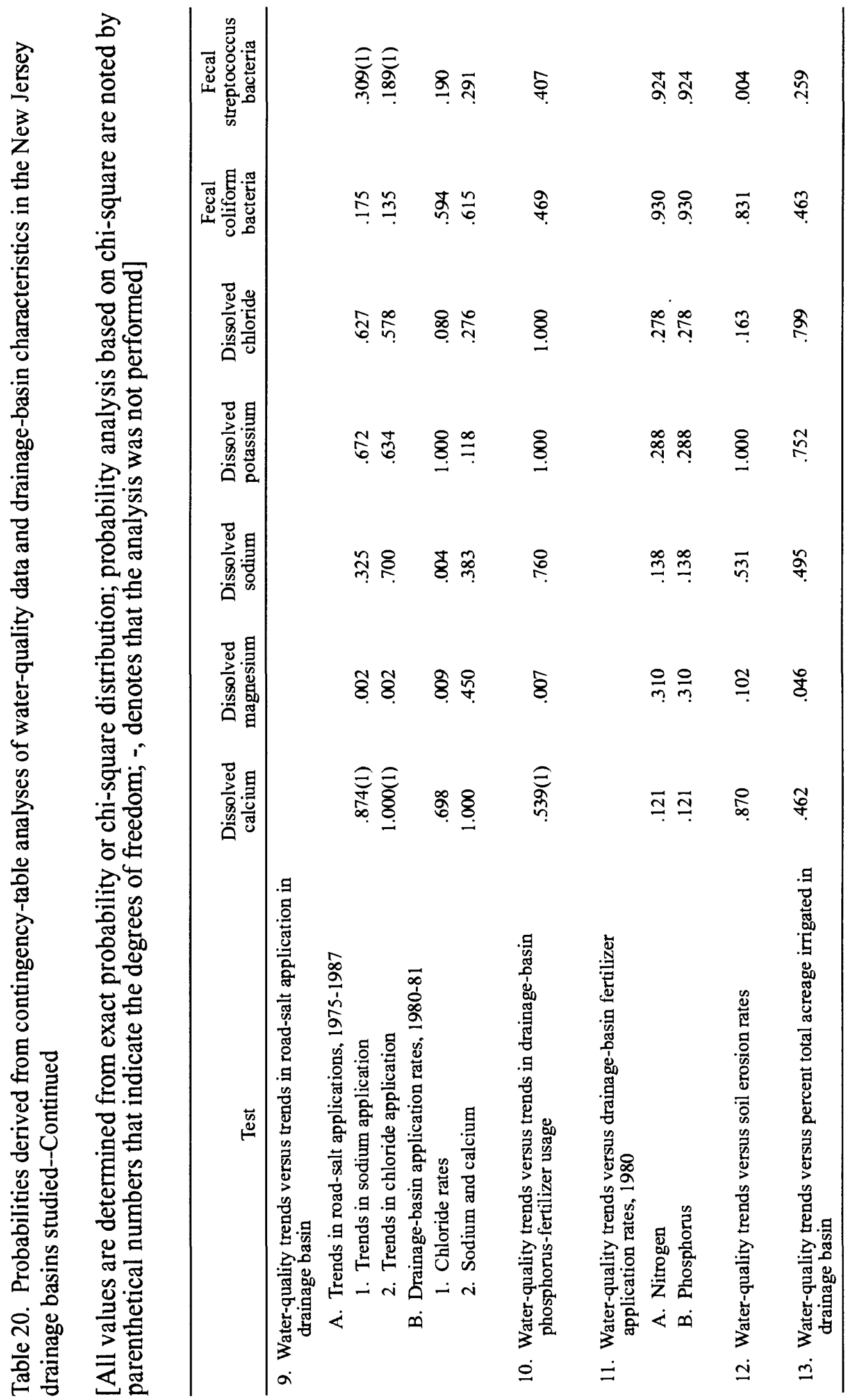


Table 21. Results of Spearman-rho correlation analyses of water-quality data from paired monitoring stations on the same New Jersey stream, water year 1976-86

$[-$, correlation analysis not performed because of lack of data $]$

\begin{tabular}{|c|c|c|c|c|c|c|}
\hline \multicolumn{2}{|r|}{ Constituent or property } & \multirow{2}{*}{\multicolumn{2}{|c|}{ Paired monitoring stations }} & \multirow{2}{*}{$\begin{array}{l}\text { Number of } \\
\text { paired values } \\
\text { for test }\end{array}$} & \multirow{2}{*}{$\begin{array}{l}\text { Correlation } \\
\text { coefficient }\end{array}$} & \multirow[b]{2}{*}{ Probability } \\
\hline Code & Name & & & & & \\
\hline 00095 & Specific conductance & 01367700 & 01367770 & 37 & 0.955 & 0.000 \\
\hline 00095 & Specific conductance & 01379000 & 01379500 & 56 & 0.563 & 0.000 \\
\hline 00095 & Specific conductance & 01379500 & 01382000 & 0 & - & - \\
\hline 00095 & Specific conductance & 01381500 & 01381800 & 7 & 0.516 & 0.236 \\
\hline 00095 & Specific conductance & 01396280 & 01396535 & 41 & 0.965 & 0.000 \\
\hline 00095 & Specific conductance & 01396535 & 01397400 & 13 & 0.666 & 0.013 \\
\hline 00095 & Specific conductance & 01397400 & 01396280 & 10 & 0.437 & 0.207 \\
\hline 00095 & Specific conductance & 01398260 & 01399120 & 17 & 0.848 & 0.000 \\
\hline 00095 & Specific conductance & 01401440 & 01402540 & 6 & 0.918 & 0.010 \\
\hline 00095 & Specific conductance & 01410784 & 01410820 & 62 & 0.658 & 0.000 \\
\hline 00095 & Specific conductance & 01410820 & 01411110 & 11 & 0.494 & 0.122 \\
\hline 00095 & Specific conductance & 01411110 & 01410784 & 10 & 0.071 & 0.845 \\
\hline 00095 & Specific conductance & 01456200 & 01457400 & 6 & 0.045 & 0.933 \\
\hline 00095 & Specific conductance & 01465970 & 01467000 & 39 & 0.719 & 0.000 \\
\hline 00095 & Specific conductance & 01467120 & 01467140 & 26 & -0.530 & 0.005 \\
\hline 00300 & Dissolved oxygen & 01367700 & 01367770 & 38 & 0.848 & 0.000 \\
\hline 00300 & Dissolved oxygen & 01379000 & 01379500 & 56 & 0.883 & 0.000 \\
\hline 00300 & Dissolved oxygen & 01379500 & 01382000 & 0 & - & - \\
\hline 00300 & Dissolved oxygen & 01381500 & 01381800 & 7 & 0.176 & 0.706 \\
\hline 00300 & Dissolved oxygen & 01396280 & 01396535 & 40 & 0.912 & 0.000 \\
\hline 00300 & Dissolved oxygen & 01396535 & 01397400 & 13 & 0.862 & 0.000 \\
\hline 00300 & Dissolved oxygen & 01397400 & 01396280 & 10 & 0.758 & 0.011 \\
\hline 00300 & Dissolved oxygen & 01398260 & 01399120 & 16 & 0.696 & 0.003 \\
\hline 00300 & Dissolved oxygen & 01401440 & 01402540 & 6 & 0.034 & 0.950 \\
\hline 00300 & Dissolved oxygen & 01410784 & 01410820 & 63 & 0.784 & 0.000 \\
\hline 00300 & Dissolved oxygen & 01410820 & 01411110 & 11 & 0.919 & 0.000 \\
\hline 00300 & Dissolved oxygen & 01411110 & 01410784 & 11 & 0.764 & 0.006 \\
\hline 00300 & Dissolved oxygen & 01456200 & 01457400 & 5 & 0.981 & 0.003 \\
\hline 00300 & Dissolved oxygen & 01465970 & 01467000 & 39 & 0.954 & 0.000 \\
\hline 00300 & Dissolved oxygen & 01467120 & 01467140 & 25 & 0.840 & 0.000 \\
\hline 00310 & Biochemical oxygen demand & 01367700 & 01367770 & 37 & 0.587 & 0.000 \\
\hline 00310 & Biochemical oxygen demand & 01379000 & 01379500 & 54 & 0.292 & 0.032 \\
\hline 00310 & Biochemical oxygen demand & 01379500 & 01382000 & $\mathbf{0}$ & - & - \\
\hline 00310 & Biochemical oxygen demand & 01381500 & 01381800 & 7 & 0.800 & 0.031 \\
\hline 00310 & Biochemical oxygen demand & 01396280 & 01396535 & 37 & 0.501 & 0.002 \\
\hline 00310 & Biochemical oxygen demand & 01396535 & 01397400 & 12 & 0.394 & 0.205 \\
\hline 00310 & Biochemical oxygen demand & 01397400 & 01396280 & 10 & 0.605 & 0.064 \\
\hline 00310 & Biochemical oxygen demand & 01398260 & 01399120 & 14 & 0.051 & 0.863 \\
\hline 00310 & Biochemical oxygen demand & 01401440 & 01402540 & 6 & 0.778 & 0.068 \\
\hline 00310 & Biochemical oxygen demand & 01410784 & 01410820 & 59 & 0.164 & 0.214 \\
\hline
\end{tabular}


Table 21. Results of Spearman-rho correlation analyses of water-quality data from paired monitoring stations on the same New Jersey stream, water year 1976-86--Continued

\begin{tabular}{|c|c|c|c|c|c|c|}
\hline \multicolumn{2}{|r|}{ Constituent or property } & \multirow{2}{*}{\multicolumn{2}{|c|}{ Paired monitoring stations }} & \multirow{2}{*}{$\begin{array}{l}\text { Number of } \\
\text { paired values } \\
\text { for test }\end{array}$} & \multirow{2}{*}{$\begin{array}{l}\text { Correlation } \\
\text { coefficient }\end{array}$} & \multirow[b]{2}{*}{ Probability } \\
\hline Code & Name & & & & & \\
\hline 00310 & Biochemical oxygen demand & 01410820 & 01411110 & 11 & 0.270 & 0.422 \\
\hline 00310 & Biochemical oxygen demand & 01411110 & 01410784 & 11 & 0.305 & 0.362 \\
\hline 00310 & Biochemical oxygen demand & 01456200 & 01457400 & 4 & - & - \\
\hline 00310 & Biochemical oxygen demand & 01465970 & 01467000 & 38 & 0.608 & 0.000 \\
\hline 00310 & Biochemical oxygen demand & 01467120 & 01467140 & 24 & -0.495 & 0.014 \\
\hline 00400 & $\mathrm{pH}$ & 01367700 & 01367770 & 37 & 0.697 & 0.000 \\
\hline 00400 & $\mathrm{pH}$ & 01379000 & 01379500 & 56 & 0.541 & 0.000 \\
\hline 00400 & $\mathrm{pH}$ & 01379500 & 01382000 & 0 & - & - \\
\hline 00400 & $\mathrm{pH}$ & 01381500 & 01381800 & 7 & 0.555 & 0.196 \\
\hline 00400 & $\mathrm{pH}$ & 01396280 & 01396535 & 42 & 0.851 & 0.000 \\
\hline 00400 & $\mathrm{pH}$ & 01396535 & 01397400 & 14 & -0.410 & 0.145 \\
\hline 00400 & $\mathrm{pH}$ & 01397400 & 01396280 & 11 & -0.504 & 0.114 \\
\hline 00400 & $\mathrm{pH}$ & 01398260 & 01399120 & 16 & 0.390 & 0.135 \\
\hline 00400 & $\mathrm{pH}$ & 01401440 & 01402540 & 6 & 0.949 & 0.004 \\
\hline 00400 & $\mathrm{pH}$ & 01410784 & 01410820 & 59 & 0.836 & 0.000 \\
\hline 00400 & $\mathrm{pH}$ & 01410820 & 01411110 & 11 & 0.952 & 0.000 \\
\hline 00400 & $\mathrm{pH}$ & 01411110 & 01410784 & 11 & 0.743 & 0.009 \\
\hline 00400 & $\mathrm{pH}$ & 01456200 & 01457400 & 6 & -0.210 & 0.690 \\
\hline 00400 & $\mathrm{pH}$ & 01465970 & 01467000 & 38 & 0.798 & 0.000 \\
\hline 00400 & $\mathrm{pH}$ & 01467120 & 01467140 & 26 & 0.073 & 0.725 \\
\hline 00600 & Total nitrogen & 01367700 & 01367770 & 33 & 0.438 & 0.011 \\
\hline 00600 & Total nitrogen & 01379000 & 01379500 & 35 & 0.277 & 0.108 \\
\hline 00600 & Total nitrogen & 01379500 & 01382000 & 0 & - & - \\
\hline 00600 & Total nitrogen & 01381500 & 01381800 & 5 & -0.967 & 0.007 \\
\hline 00600 & Total nitrogen & 01396280 & 01396535 & 36 & 0.523 & 0.001 \\
\hline 00600 & Total nitrogen & 01396535 & 01397400 & 12 & -0.263 & 0.408 \\
\hline 00600 & Total nitrogen & 01397400 & 01396280 & 8 & -0.451 & 0.262 \\
\hline 00600 & Total nitrogen & 01398260 & 01399120 & 12 & 0.456 & 0.136 \\
\hline 00600 & Total nitrogen & 01401440 & 01402540 & 4 & - & - \\
\hline 00600 & Total nitrogen & 01410784 & 01410820 & 53 & 0.730 & 0.000 \\
\hline 00600 & Total nitrogen & 01410820 & 01411110 & 9 & 0.666 & 0.050 \\
\hline 00600 & Total nitrogen & 01411110 & 01410784 & 10 & 0.265 & 0.459 \\
\hline 00600 & Total nitrogen & 01456200 & 01457400 & 5 & 0.425 & 0.476 \\
\hline 00600 & Total nitrogen & 01465970 & 01467000 & 18 & 0.372 & 0.129 \\
\hline 00600 & Total nitrogen & 01467120 & 01467140 & 11 & 0.383 & 0.245 \\
\hline 00610 & Total ammonia & 01367700 & 01367770 & 36 & 0.301 & 0.074 \\
\hline 00610 & Total ammonia & 01379000 & 01379500 & 47 & 0.218 & 0.141 \\
\hline 00610 & Total ammonia & 01379500 & 01382000 & 0 & - & - \\
\hline 00610 & Total ammonia & 01381500 & 01381800 & 6 & -0.205 & 0.696 \\
\hline 00610 & Total ammonia & 01396280 & 01396535 & 37 & 0.132 & 0.437 \\
\hline
\end{tabular}


Table 21. Results of Spearman-rho correlation analyses of water-quality data from paired monitoring stations on the same New Jersey stream, water year 1976-86--Continued

\begin{tabular}{|c|c|c|c|c|c|c|}
\hline \multicolumn{2}{|r|}{ Constituent or property } & \multirow{2}{*}{\multicolumn{2}{|c|}{ Paired monitoring stations }} & \multirow{2}{*}{$\begin{array}{l}\text { Number of } \\
\text { paired values } \\
\text { for test }\end{array}$} & \multirow{2}{*}{$\begin{array}{l}\text { Correlation } \\
\text { coefficient }\end{array}$} & \multirow[b]{2}{*}{ Probability } \\
\hline Code & Name & & & & & \\
\hline 00610 & Total ammonia & 01396535 & 01397400 & 12 & -0.091 & 0.779 \\
\hline 00610 & Total ammonia & 01397400 & 01396280 & 9 & -0.122 & 0.754 \\
\hline 00610 & Total ammonia & 01398260 & 01399120 & 16 & -0.121 & 0.656 \\
\hline 00610 & Total ammonia & 01401440 & 01402540 & 4 & - & - \\
\hline 00610 & Total ammonia & 01410784 & 01410820 & 60 & 0.561 & 0.000 \\
\hline 00610 & Total ammonia & 01410820 & 01411110 & 10 & 0.715 & 0.020 \\
\hline 00610 & Total ammonia & 01411110 & 01410784 & 11 & 0.345 & 0.299 \\
\hline 00610 & Total ammonia & 01456200 & 01457400 & 5 & 0.924 & 0.025 \\
\hline 00610 & Total ammonia & 01465970 & 01467000 & 30 & 0.899 & 0.000 \\
\hline 00610 & Total ammonia & 01467120 & 01467140 & 19 & 0.168 & 0.492 \\
\hline 00665 & Total phosphorus & 01367700 & 01367770 & 36 & 0.384 & 0.021 \\
\hline 00665 & Total phosphorus & 01379000 & 01379500 & 51 & 0.576 & 0.000 \\
\hline 00665 & Total phosphorus & 01379500 & 01382000 & 0 & - & - \\
\hline 00665 & Total phosphorus & 01381500 & 01381800 & 5 & 0.730 & 0.161 \\
\hline 00665 & Total phosphorus & 01396280 & 01396535 & 41 & 0.603 & 0.000 \\
\hline 00665 & Total phosphorus & 01396535 & 01397400 & 13 & 0.102 & 0.739 \\
\hline 00665 & Total phosphorus & 01397400 & 01396280 & 10 & 0.204 & 0.572 \\
\hline 00665 & Total phosphorus & 01398260 & 01399120 & 16 & 0.649 & 0.006 \\
\hline 00665 & Total phosphorus & 01401440 & 01402540 & 5 & -0.636 & 0.249 \\
\hline 00665 & Total phosphorus & 01410784 & 01410820 & 59 & 0.668 & 0.000 \\
\hline 00665 & Total phosphorus & 01410820 & 01411110 & 10 & 0.529 & 0.116 \\
\hline 00665 & Total phosphorus & 01411110 & 01410784 & 11 & 0.544 & 0.084 \\
\hline 00665 & Total phosphorus & 01456200 & 01457400 & 6 & -0.095 & 0.859 \\
\hline 00665 & Total phosphorus & 01465970 & 01467000 & 39 & 0.477 & 0.002 \\
\hline 00665 & Total phosphorus & 01467120 & 01467140 & 25 & 0.340 & 0.096 \\
\hline 00680 & Total organic carbon & 01367700 & 01367770 & 35 & 0.610 & 0.000 \\
\hline 00680 & Total organic carbon & 01379000 & 01379500 & 53 & 0.448 & 0.001 \\
\hline 00680 & Total organic carbon & 01379500 & 01382000 & 0 & - & - \\
\hline 00680 & Total organic carbon & 01381500 & 01381800 & 7 & -0.645 & 0.118 \\
\hline 00680 & Total organic carbon & 01396280 & 01396535 & 40 & 0.602 & 0.000 \\
\hline 00680 & Total organic carbon & 01396535 & 01397400 & 12 & 0.373 & 0.233 \\
\hline 00680 & Total organic carbon & 01397400 & 01396280 & 9 & 0.034 & 0.931 \\
\hline 00680 & Total organic carbon & 01398260 & 01399120 & 16 & 0.850 & 0.000 \\
\hline 00680 & Total organic carbon & 01401440 & 01402540 & 4 & - & - \\
\hline 00680 & Total organic carbon & 01410784 & 01410820 & 61 & 0.804 & 0.000 \\
\hline 00680 & Total organic carbon & 01410820 & 01411110 & 11 & 0.834 & 0.001 \\
\hline 00680 & Total organic carbon & 01411110 & 01410784 & 11 & 0.834 & 0.001 \\
\hline 00680 & Total organic carbon & 01456200 & 01457400 & 5 & -0.361 & 0.550 \\
\hline 00680 & Total organic carbon & 01465970 & 01467000 & 36 & 0.703 & 0.000 \\
\hline 00680 & Total organic carbon & 01467120 & 01467140 & 25 & 0.006 & 0.976 \\
\hline
\end{tabular}


Table 21. Results of Spearman-rho correlation analyses of water-quality data from paired monitoring stations on the same New Jersey stream, water year 1976-86--Continued

\begin{tabular}{|c|c|c|c|c|c|c|}
\hline \multicolumn{2}{|r|}{ Constituent or property } & \multirow{2}{*}{\multicolumn{2}{|c|}{ Paired monitoring stations }} & \multirow{2}{*}{$\begin{array}{l}\text { Number of } \\
\text { paired values } \\
\text { for test }\end{array}$} & \multirow{2}{*}{$\begin{array}{l}\text { Correlation } \\
\text { coefficient }\end{array}$} & \multirow[b]{2}{*}{ Probability } \\
\hline Code & Name & & & & & \\
\hline 00915 & Dissolved calcium & 01367700 & 01367770 & 37 & 0.951 & 0.000 \\
\hline 00915 & Dissolved calcium & 01379000 & 01379500 & 56 & 0.774 & 0.000 \\
\hline 00915 & Dissolved calcium & 01379500 & 01382000 & 0 & - & - \\
\hline 00915 & Dissolved calcium & 01381500 & 01381800 & 7 & 0.275 & 0.551 \\
\hline 00915 & Dissolved calcium & 01396280 & 01396535 & 40 & 0.494 & 0.001 \\
\hline 00915 & Dissolved calcium & 01396535 & 01397400 & 13 & 0.501 & 0.081 \\
\hline 00915 & Dissolved calcium & 01397400 & 01396280 & 10 & -0.275 & 0.442 \\
\hline 00915 & Dissolved calcium & 01398260 & 01399120 & 17 & 0.816 & 0.000 \\
\hline 00915 & Dissolved calcium & 01401440 & 01402540 & 6 & 0.386 & 0.449 \\
\hline 00915 & Dissolved calcium & 01410784 & 01410820 & 51 & 0.526 & 0.000 \\
\hline 00915 & Dissolved calcium & 01410820 & 01411110 & 10 & 0.969 & 0.000 \\
\hline 00915 & Dissolved calcium & 01411110 & 01410784 & 11 & -0.574 & 0.065 \\
\hline 00915 & Dissolved calcium & 01456200 & 01457400 & 6 & 0.628 & 0.182 \\
\hline 00915 & Dissolved calcium & 01465970 & 01467000 & 38 & 0.706 & 0.000 \\
\hline 00915 & Dissolved calcium & 01467120 & 01467140 & 26 & 0.152 & 0.459 \\
\hline 00925 & Dissolved magnesium & 01367700 & 01367770 & 37 & 0.966 & 0.000 \\
\hline 00925 & Dissolved magnesium & 01379000 & 01379500 & 56 & 0.818 & 0.000 \\
\hline 00925 & Dissolved magnesium & 01379500 & 01382000 & 0 & - & - \\
\hline 00925 & Dissolved magnesium & 01381500 & 01381800 & 7 & 0.606 & 0.149 \\
\hline 00925 & Dissolved magnesium & 01396280 & 01396535 & 40 & 0.260 & 0.105 \\
\hline 00925 & Dissolved magnesium & 01396535 & 01397400 & 13 & 0.059 & 0.848 \\
\hline 00925 & Dissolved magnesium & 01397400 & 01396280 & 10 & 0.084 & 0.817 \\
\hline 00925 & Dissolved magnesium & 01398260 & 01399120 & 17 & 0.894 & 0.000 \\
\hline 00925 & Dissolved magnesium & 01401440 & 01402540 & 6 & 0.480 & 0.336 \\
\hline 00925 & Dissolved magnesium & 01410784 & 01410820 & 51 & 0.771 & 0.000 \\
\hline 00925 & Dissolved magnesium & 01410820 & 01411110 & 10 & 0.722 & 0.018 \\
\hline 00925 & Dissolved magnesium & 01411110 & 01410784 & 11 & 0.069 & 0.841 \\
\hline 00925 & Dissolved magnesium & 01456200 & 01457400 & 6 & -0.083 & 0.875 \\
\hline 00925 & Dissolved magnesium & 01465970 & 01467000 & 38 & 0.769 & 0.000 \\
\hline 00925 & Dissolved magnesium & 01467120 & 01467140 & 26 & 0.213 & 0.297 \\
\hline 00930 & Dissolved sodium & 01367700 & 01367770 & 37 & 0.871 & 0.000 \\
\hline 00930 & Dissolved sodium & 01379000 & 01379500 & 56 & 0.253 & 0.060 \\
\hline 00930 & Dissolved sodium & 01379500 & 01382000 & 0 & - & - \\
\hline 00930 & Dissolved sodium & 01381500 & 01381800 & 7 & 0.576 & 0.175 \\
\hline 00930 & Dissolved sodium & 01396280 & 01396535 & 40 & 0.519 & 0.001 \\
\hline 00930 & Dissolved sodium & 01396535 & 01397400 & 13 & 0.292 & 0.333 \\
\hline 00930 & Dissolved sodium & 01397400 & 01396280 & 10 & -0.289 & 0.417 \\
\hline 00930 & Dissolved sodium & 01398260 & 01399120 & 17 & 0.817 & 0.000 \\
\hline 00930 & Dissolved sodium & 01401440 & 01402540 & 6 & 0.522 & 0.288 \\
\hline 00930 & Dissolved sodium & 01410784 & 01410820 & 52 & 0.758 & 0.000 \\
\hline
\end{tabular}


Table 21. Results of Spearman-rho correlation analyses of water-quality data from paired monitoring stations on the same New Jersey stream, water year 1976-86--Continued

\begin{tabular}{|c|c|c|c|c|c|c|}
\hline \multicolumn{2}{|r|}{ Constituent or property } & \multirow{2}{*}{\multicolumn{2}{|c|}{ Paired monitoring stations }} & \multirow{2}{*}{$\begin{array}{l}\text { Number of } \\
\text { paired values } \\
\text { for test }\end{array}$} & \multirow{2}{*}{$\begin{array}{c}\text { Correlation } \\
\text { coefficient }\end{array}$} & \multirow[b]{2}{*}{ Probability } \\
\hline Code & Name & & & & & \\
\hline 00930 & Dissolved sodium & 01410820 & 01411110 & 10 & 0.685 & 0.029 \\
\hline 00930 & Dissolved sodium & 01411110 & 01410784 & 11 & 0.244 & 0.469 \\
\hline 00930 & Dissolved sodium & 01456200 & 01457400 & 6 & -0.062 & 0.907 \\
\hline 00930 & Dissolved sodium & 01465970 & 01467000 & 38 & 0.777 & 0.000 \\
\hline 00930 & Dissolved sodium & 01467120 & 01467140 & 26 & 0.074 & 0.719 \\
\hline 00935 & Dissolved potassium & 01367700 & 01367770 & 37 & 0.887 & 0.000 \\
\hline 00935 & Dissolved potassium & 01379000 & 01379500 & 56 & 0.316 & 0.018 \\
\hline 00935 & Dissolved potassium & 01379500 & 01382000 & 0 & - & - \\
\hline 00935 & Dissolved potassium & 01381500 & 01381800 & 7 & 0.330 & 0.470 \\
\hline 00935 & Dissolved potassium & 01396280 & 01396535 & 40 & 0.472 & 0.002 \\
\hline 00935 & Dissolved potassium & 01396535 & 01397400 & 13 & 0.643 & 0.018 \\
\hline 00935 & Dissolved potassium & 01397400 & 01396280 & 10 & -0.110 & 0.762 \\
\hline 00935 & Dissolved potassium & 01398260 & 01399120 & 17 & 0.812 & 0.000 \\
\hline 00935 & Dissolved potassium & 01401440 & 01402540 & 6 & 0.120 & 0.821 \\
\hline 00935 & Dissolved potassium & 01410784 & 01410820 & 52 & 0.602 & 0.000 \\
\hline 00935 & Dissolved potassium & 01410820 & 01411110 & 10 & 0.698 & 0.025 \\
\hline 00935 & Dissolved potassium & 01411110 & 01410784 & 11 & 0.245 & 0.467 \\
\hline 00935 & Dissolved potassium & 01456200 & 01457400 & 6 & 0.783 & 0.066 \\
\hline 00935 & Dissolved potassium & 01465970 & 01467000 & 38 & 0.809 & 0.000 \\
\hline 00935 & Dissolved potassium & 01467120 & 01467140 & 26 & -0.146 & 0.477 \\
\hline 00940 & Dissolved chloride & 01367700 & 01367770 & 37 & 0.905 & 0.000 \\
\hline 00940 & Dissolved chloride & 01379000 & 01379500 & 56 & 0.293 & 0.029 \\
\hline 00940 & Dissolved chloride & 01379500 & 01382000 & 0 & - & - \\
\hline 00940 & Dissolved chloride & 01381500 & 01381800 & 7 & 0.640 & 0.121 \\
\hline 00940 & Dissolved chloride & 01396280 & 01396535 & 39 & 0.970 & 0.000 \\
\hline 00940 & Dissolved chloride & 01396535 & 01397400 & 13 & 0.770 & 0.002 \\
\hline 00940 & Dissolved chloride & 01397400 & 01396280 & 9 & 0.581 & 0.101 \\
\hline 00940 & Dissolved chloride & 01398260 & 01399120 & 17 & 0.864 & 0.000 \\
\hline 00940 & Dissolved chloride & 01401440 & 01402540 & 6 & 0.440 & 0.383 \\
\hline 00940 & Dissolved chloride & 01410784 & 01410820 & 52 & 0.767 & 0.000 \\
\hline 00940 & Dissolved chloride & 01410820 & 01411110 & 10 & 0.966 & 0.000 \\
\hline 00940 & Dissolved chloride & 01411110 & 01410784 & 11 & -0.081 & 0.812 \\
\hline 00940 & Dissolved chloride & 01456200 & 01457400 & 6 & -0.073 & 0.890 \\
\hline 00940 & Dissolved chloride & 01465970 & 01467000 & 38 & 0.750 & 0.000 \\
\hline 00940 & Dissolved chloride & 01467120 & 01467140 & 26 & 0.026 & 0.898 \\
\hline 31615 & Fecal coliform bacteria & 01367700 & 01367770 & 37 & 0.202 & 0.230 \\
\hline 31615 & Fecal coliform bacteria & 01379000 & 01379500 & 55 & 0.848 & 0.000 \\
\hline 31615 & Fecal coliform bacteria & 01379500 & 01382000 & 0 & - & - \\
\hline 31615 & Fecal coliform bacteria & 01381500 & 01381800 & 7 & -0.214 & 0.645 \\
\hline 31615 & Fecal coliform bacteria & 01396280 & 01396535 & 38 & 0.704 & 0.000 \\
\hline
\end{tabular}


Table 21. Results of Spearman-rho correlation analyses of water-quality data from paired monitoring stations on the same New Jersey stream, water year 1976-86--Continued

\begin{tabular}{|c|c|c|c|c|c|c|}
\hline \multicolumn{2}{|r|}{ Constituent or property } & \multirow{2}{*}{\multicolumn{2}{|c|}{ Paired monitoring stations }} & \multirow{2}{*}{$\begin{array}{l}\text { Number of } \\
\text { paired values } \\
\text { for test }\end{array}$} & \multirow{2}{*}{$\begin{array}{c}\text { Correlation } \\
\text { coefficient }\end{array}$} & \multirow[b]{2}{*}{ Probability } \\
\hline Code & Name & & & & & \\
\hline 31615 & Fecal coliform bacteria & 01396535 & 01397400 & 11 & -0.015 & 0.965 \\
\hline 31615 & Fecal coliform bacteria & 01397400 & 01396280 & 8 & 0.068 & 0.872 \\
\hline 31615 & Fecal coliform bacteria & 01398260 & 01399120 & 16 & 0.923 & 0.000 \\
\hline 31615 & Fecal coliform bacteria & 01401440 & 01402540 & 6 & 0.909 & 0.012 \\
\hline 31615 & Fecal coliform bacteria & 01410784 & 01410820 & 48 & 0.387 & 0.007 \\
\hline 31615 & Fecal coliform bacteria & 01410820 & 01411110 & 10 & 0.112 & 0.759 \\
\hline 31615 & Fecal coliform bacteria & 01411110 & 01410784 & 10 & -0.193 & 0.593 \\
\hline 31615 & Fecal coliform bacteria & 01456200 & 01457400 & 5 & 0.730 & 0.161 \\
\hline 31615 & Fecal coliform bacteria & 01465970 & 01467000 & 37 & 0.320 & 0.053 \\
\hline 31615 & Fecal coliform bacteria & 01467120 & 01467140 & 26 & 0.243 & 0.233 \\
\hline 31677 & Fecal streptococcus bacteria & 01367700 & 01367770 & 37 & 0.398 & 0.015 \\
\hline 31677 & Fecal streptococcus bacteria & 01379000 & 01379500 & 49 & 0.765 & 0.000 \\
\hline 31677 & Fecal streptococcus bacteria & 01379500 & 01382000 & 0 & - & - \\
\hline 31677 & Fecal streptococcus bacteria & 01381500 & 01381800 & 7 & 0.032 & 0.945 \\
\hline 31677 & Fecal streptococcus bacteria & 01396280 & 01396535 & 38 & 0.717 & 0.000 \\
\hline 31677 & Fecal streptococcus bacteria & 01396535 & 01397400 & 12 & 0.419 & 0.175 \\
\hline 31677 & Fecal streptococcus bacteria & 01397400 & 01396280 & 9 & 0.330 & 0.387 \\
\hline 31677 & Fecal streptococcus bacteria & 01398260 & 01399120 & 16 & 0.487 & 0.055 \\
\hline 31677 & Fecal streptococcus bacteria & 01401440 & 01402540 & 5 & -0.050 & 0.937 \\
\hline 31677 & Fecal streptococcus bacteria & 01410784 & 01410820 & 48 & 0.065 & 0.662 \\
\hline 31677 & Fecal streptococcus bacteria & 01410820 & 01411110 & 10 & 0.538 & 0.109 \\
\hline 31677 & Fecal streptococcus bacteria & 01411110 & 01410784 & 10 & 0.340 & 0.336 \\
\hline 31677 & Fecal streptococcus bacteria & 01456200 & 01457400 & 5 & 0.576 & 0.309 \\
\hline 31677 & Fecal streptococcus bacteria & 01465970 & 01467000 & 35 & 0.178 & 0.307 \\
\hline 31677 & Fecal streptococcus bacteria & 01467120 & 01467140 & 21 & 0.300 & 0.187 \\
\hline
\end{tabular}




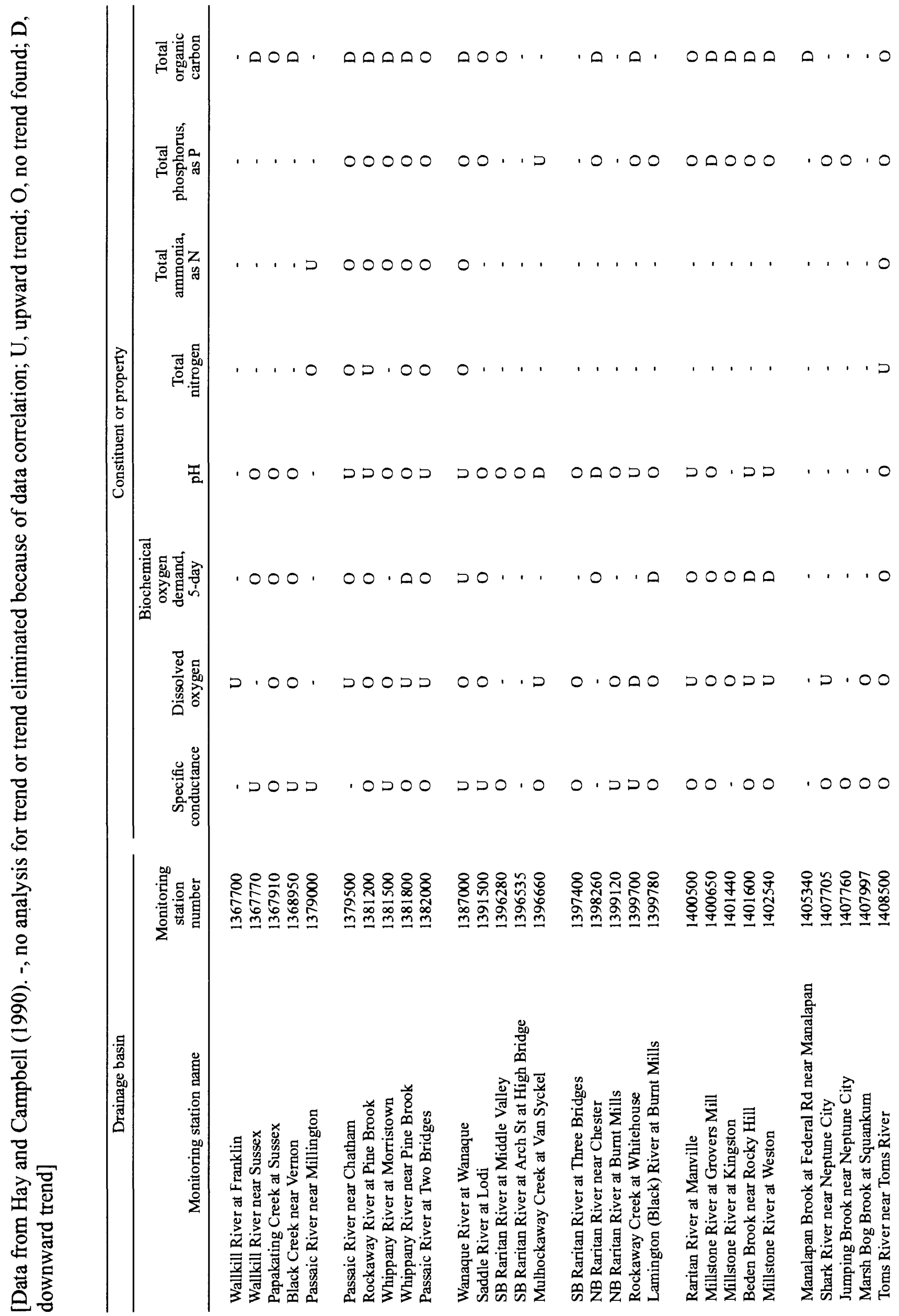

है

常营离

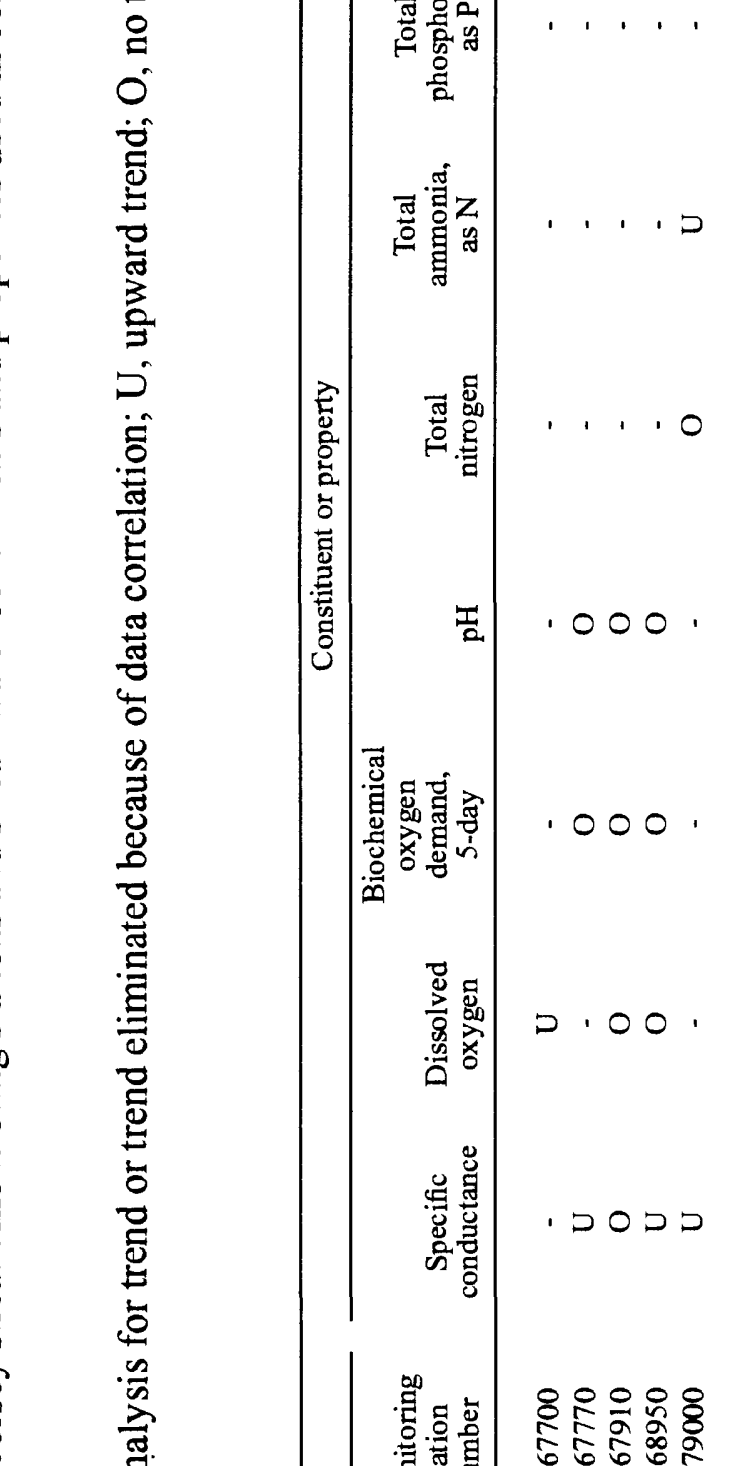

호 疍

듕 윯 荧 


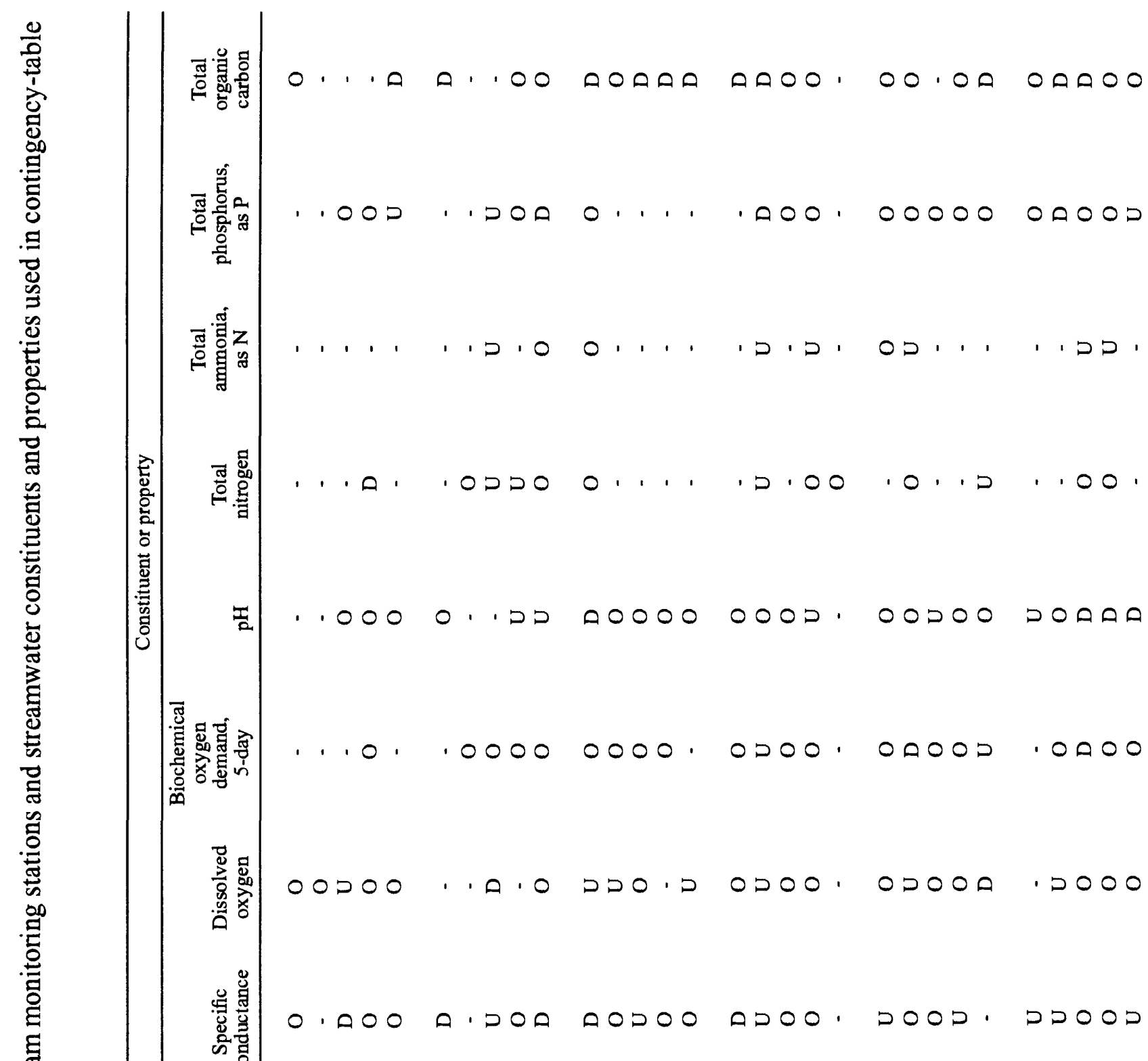

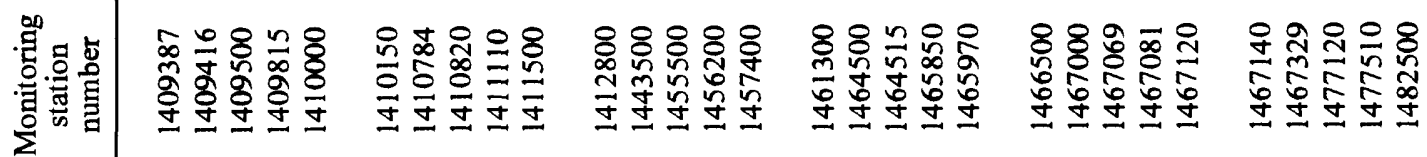

.

量若

参

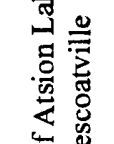

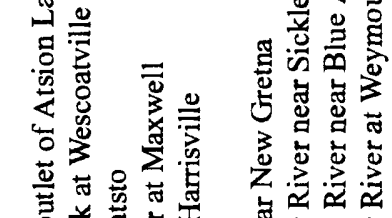

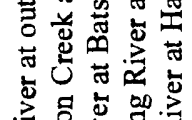

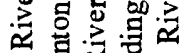

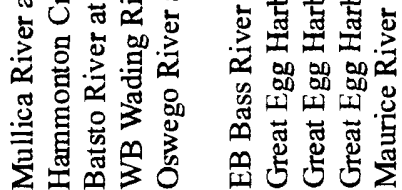

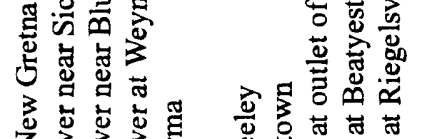
Z完空究

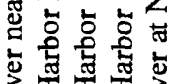

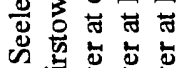

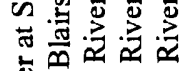

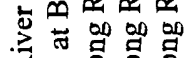

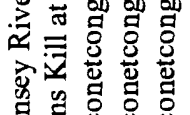

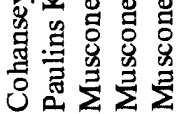

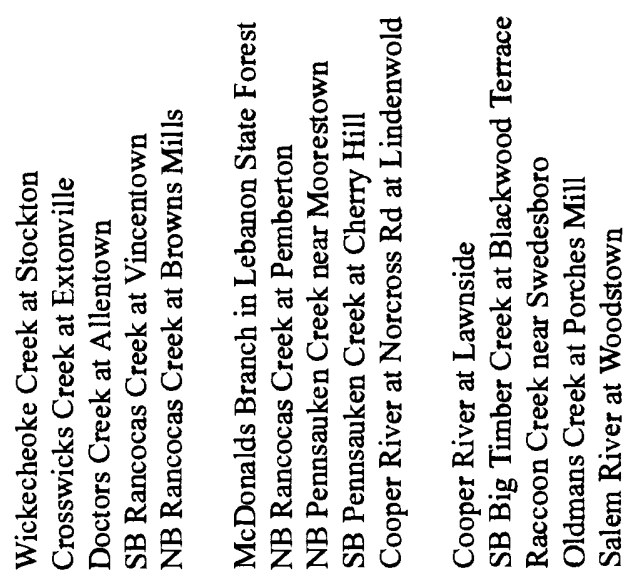




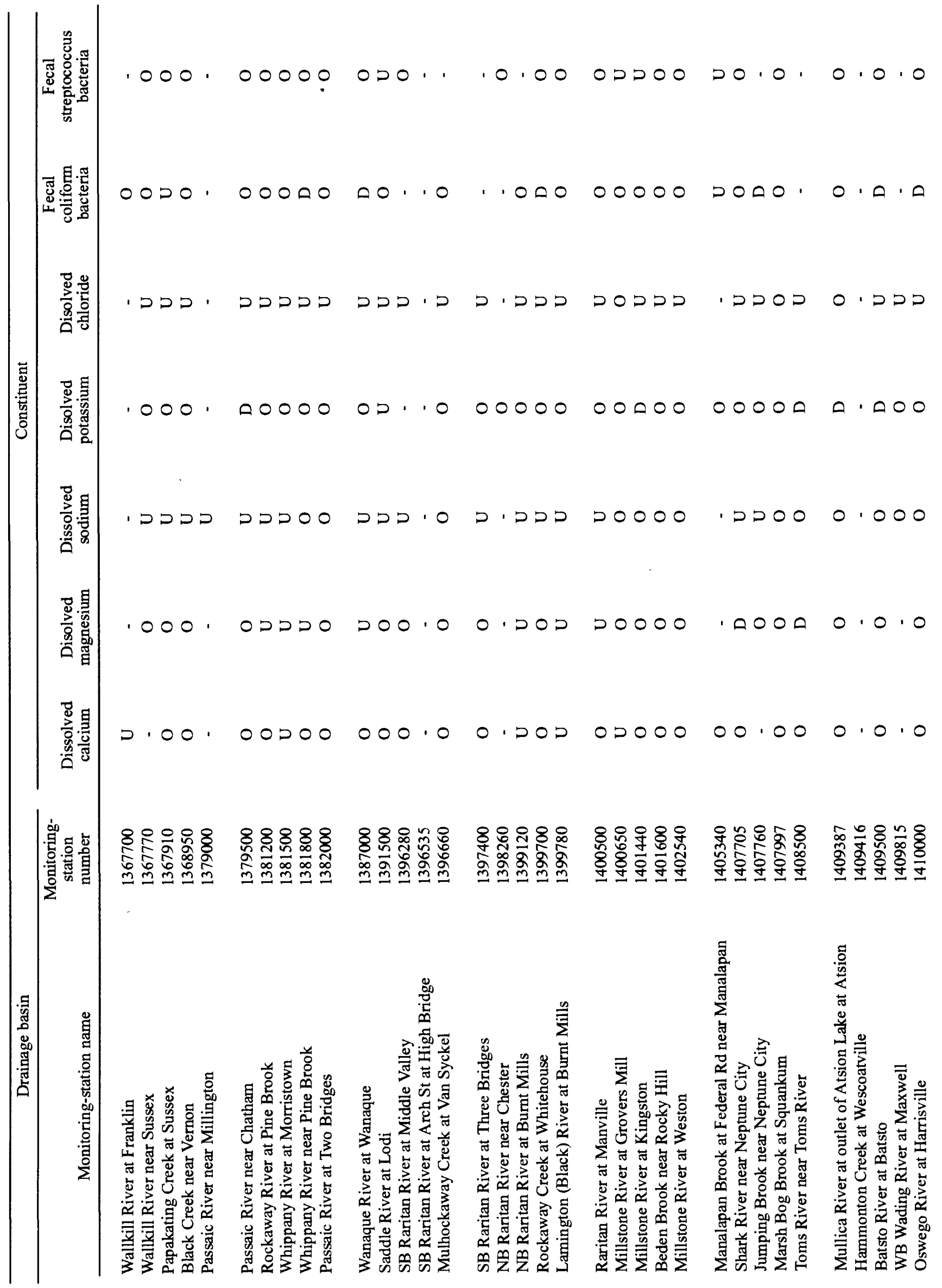




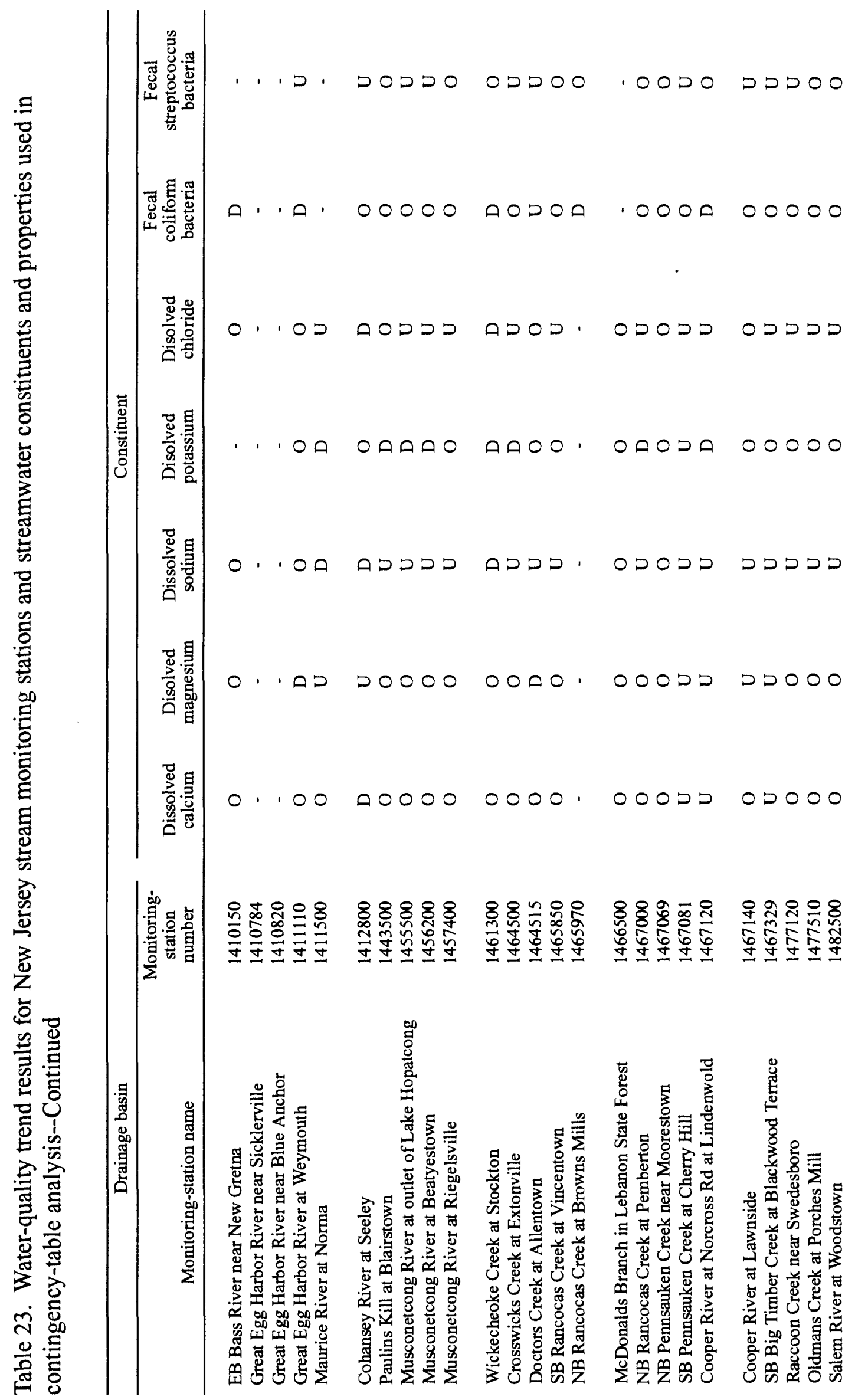

